Portland State University

PDXScholar

$1-1-1982$

\title{
Kinetics of proton transport in lipid membranes induced by some chlorinated phenols
}

Ananda Ranjith Jayaweera

Portland State University

Follow this and additional works at: https://pdxscholar.library.pdx.edu/open_access_etds Let us know how access to this document benefits you.

\section{Recommended Citation}

Jayaweera, Ananda Ranjith, "Kinetics of proton transport in lipid membranes induced by some chlorinated phenols" (1982). Dissertations and Theses. Paper 215.

https://doi.org/10.15760/etd.215

This Dissertation is brought to you for free and open access. It has been accepted for inclusion in Dissertations and Theses by an authorized administrator of PDXScholar. Please contact us if we can make this document more accessible: pdxscholar@pdx.edu. 


\section{KINETICS OF PROTON TRANSPORT IN LIPID MEMBRANES \\ INDUCED BY SOME CHLORINATED PHENOLS}

\section{by}

ANANDA RANJITH JAYAWEERA
A dissertation submitted in partial fulfillment of the requirements for the degree of

\author{
DOCTOR OF PHILOSOPHY \\ in \\ ENVIRONMENTAL SCIENCES AND RESOURCES -- PHYSICS
}

Portland State University

(C) 1982 Ananda Ranjith Jayaweera 
TO THE OFFICE OF GRADUATE STUDIES AND RESEARCH:

The members of the Committee approve the dissertation of Ananda Ranjith Jayaweera presented May 29, 1981.

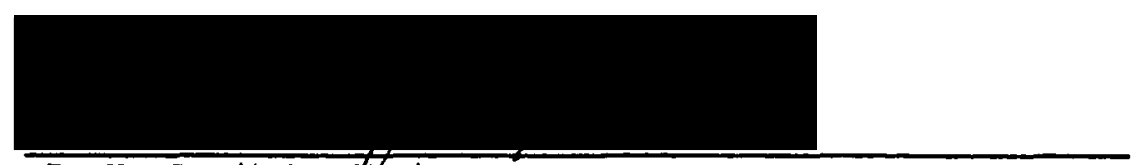

P. K. Smejtek, Ghairman

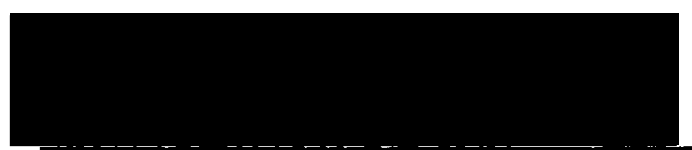

$\bar{M}$ Gurevitch
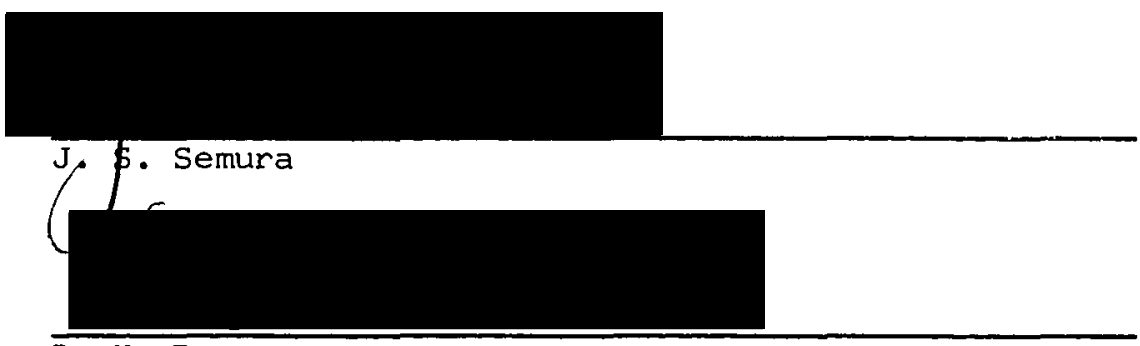

D. K. Roe

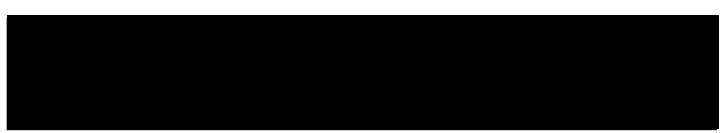

R. R. Petersen

APPROVED :

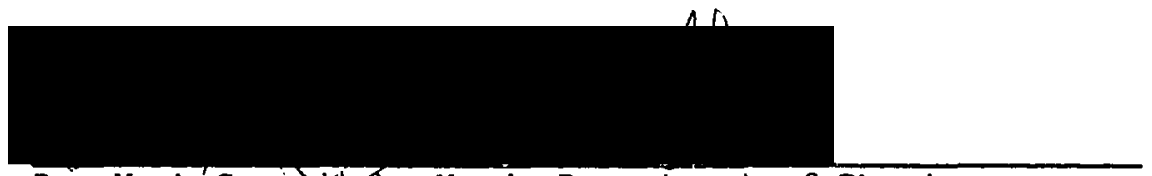

Dr. Mark Gurevitch, Head, Department of Physics

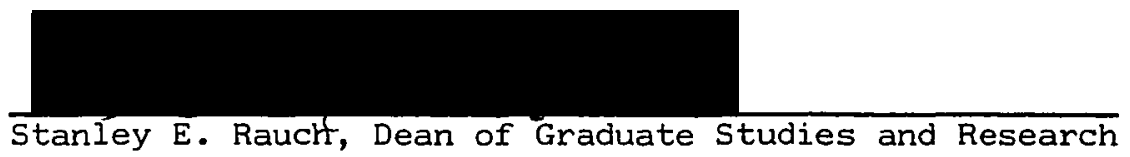


AN ABSTRACT OF THE DISSERTATION OF Ananda Ranjith Jayaweera for the Doctor of Philosophy in Environmental Sciences and Resources/Physics presented May 29, 1981.

Title: Kinetics of Proton Transport in Lipid Membranes Induced by Some Chlorinated Phenols.

APPROVED BY MEMBERS OF THE DISSERTATION COMMITTEE:
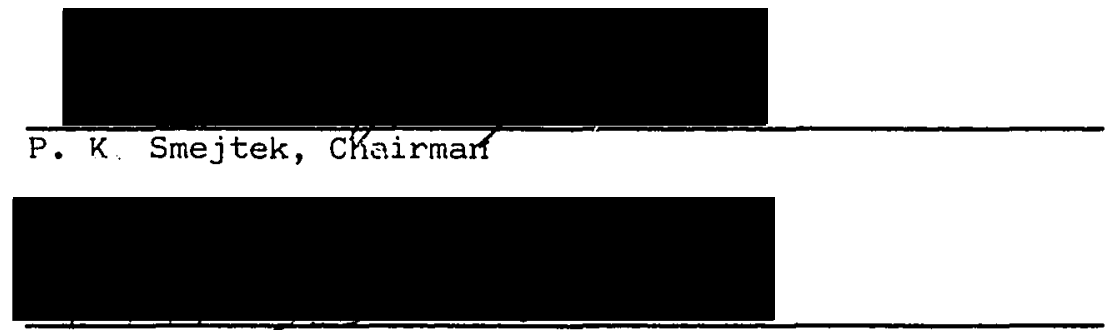

M. Gurevitch

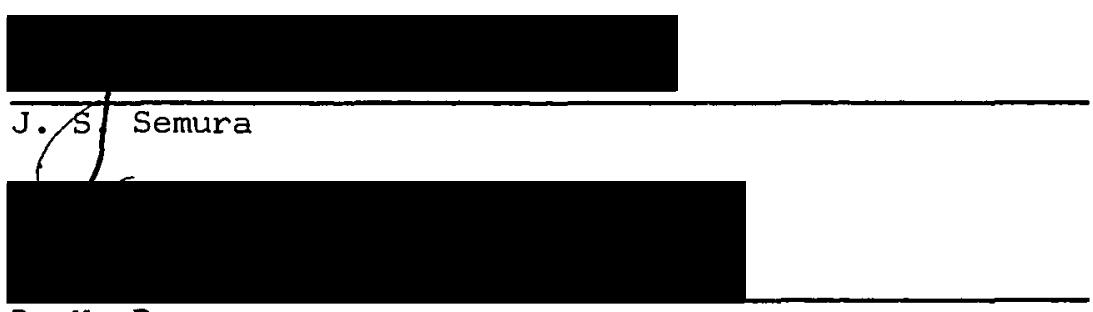

D. K. Roe

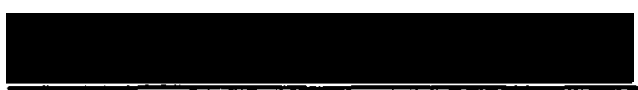

R. R. Petersen

This dissertation represents an attempt to understand the mechanism of the action of pesticides derived from chlorinated phenols in lipid membranes; specifically, the kinetics of pesticide induced hydro- 
gen ion transfer in lecithin-cholesterol membranes and its relationship to uncoupling activity in energy transducing membranes. The compounds of interest were pentachlorophenol, pentachlorobenzenethiol, and 2,4,5-trichlorophenol, which are uncouplers of oxidative phosphorylation and photosynthetic phosphorylation. Information on pesticide induced charge transport in membranes was obtained from the measurements of steady state and transient membrane conductance and membrane potentials as a function of the composition of the aqueous phase surrounding the membrane and of membrane composition. In addition, a systematic theoretical study of a series of memiorane transport models was performed in order to elucidate the various aspects of membrane transport kinetics and to make predictions relevant to the interpretation of experimental results and for further studies of membrane transport kinetics. Based on the theoretical results, two kinetic schemes of membrane transport were proposed to explain the experimental results; one for pentachlorophenol and 2,4,5-trichlorophenol, and another for pentachlorobenzenethiol. The schemes differ in the proposed mechanism of charge transfer across membrane water interface. Some conclusions regarding the rate limiting step in the charge transport process are drawn. The observation that the normalized membrane conductance and the relaxation amplitude depend on $\mathrm{pH}$ and pesticide concentration indicates that the rate limiting step of proton transport kinetics is not related to the slow rate of formation or dissociation of a membrane permeable ion. It has been shown that the negative membrane surface charge is due to the adsorption of pentachlorophenol in the ionized form. Measurements of membrane surface potentials based on the microelectrophoretic method reveal that the density of ionized pentachlorophenol at the 
membrane surface can be predicted from the Langmuir adsorption model, provided that electrostatic repulsion between pentachlorophenol ions adsorbed at the membrane and free ions in the aqueous phase is taken into account. The fact that the adsorption of positively charged tetraphenylarsonium ions and negatively charged salicylate ions modify the membrane charge transport induced by 2,4,5-trichlorophenol, can be explained by electrostatic arguments. The ability of pentachlorobenzenethiol and of chlorinated phenols to induce proton transport in membranes is regarded as the origin for both the membrane electrical conductivity and their toxic action in energy transducing membranes. We have found similarities between the $\mathrm{pH}$ dependence of pentachlorobenzenethiol induced membrane conductance and the $\mathrm{pH}$ dependence of the pentachlorobenzenethiol uncoupling activity found in mitochondria, as reported in the literature. We have also found that the level of pentachlorophenol concentration at which the membrane conductance increases above the background level corresponds to the onset of reduction of carbon uptake by algae. 
ACKNOWLEDGEMENTS

This dissertation is dedicated to my parents for their support and encouragement and for taking great pride in my achievements. I thank my wife, Amitha, for her patience and for taking care of me during many difficult times. My sincere appreciation and gratitude go to my advisor and friend Dr. Smejtek for his invaluable guidance and encouragement during the course of the research and in the preparation of the manuscript. I thank my wife for proofreading, her brother, Nimal, for the help in drawing figures, and my son, Ruwan, for being a good companion. I thank all the members of the membrane biophysics group for their helpful discussions. MY special thanks go to Dr. Abramson for being a good friend. I thank Dr. Semura for his valuable advice and the members of the dissertation committee for their cooperation.

This investigation was supported by Grant 2ROI ES 00937-04 from the National Institute of Health. 
TABLE OF CONTENTS

PAGE

ACKNOWLEDGEMENTS • • • • • • . • • • • . . • • • • •

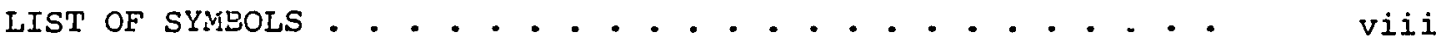

LIST OF TABLES . . . . . . . . . . . . . . . . . . . . . . x xiv

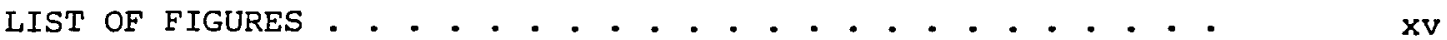

CHAPTER

I

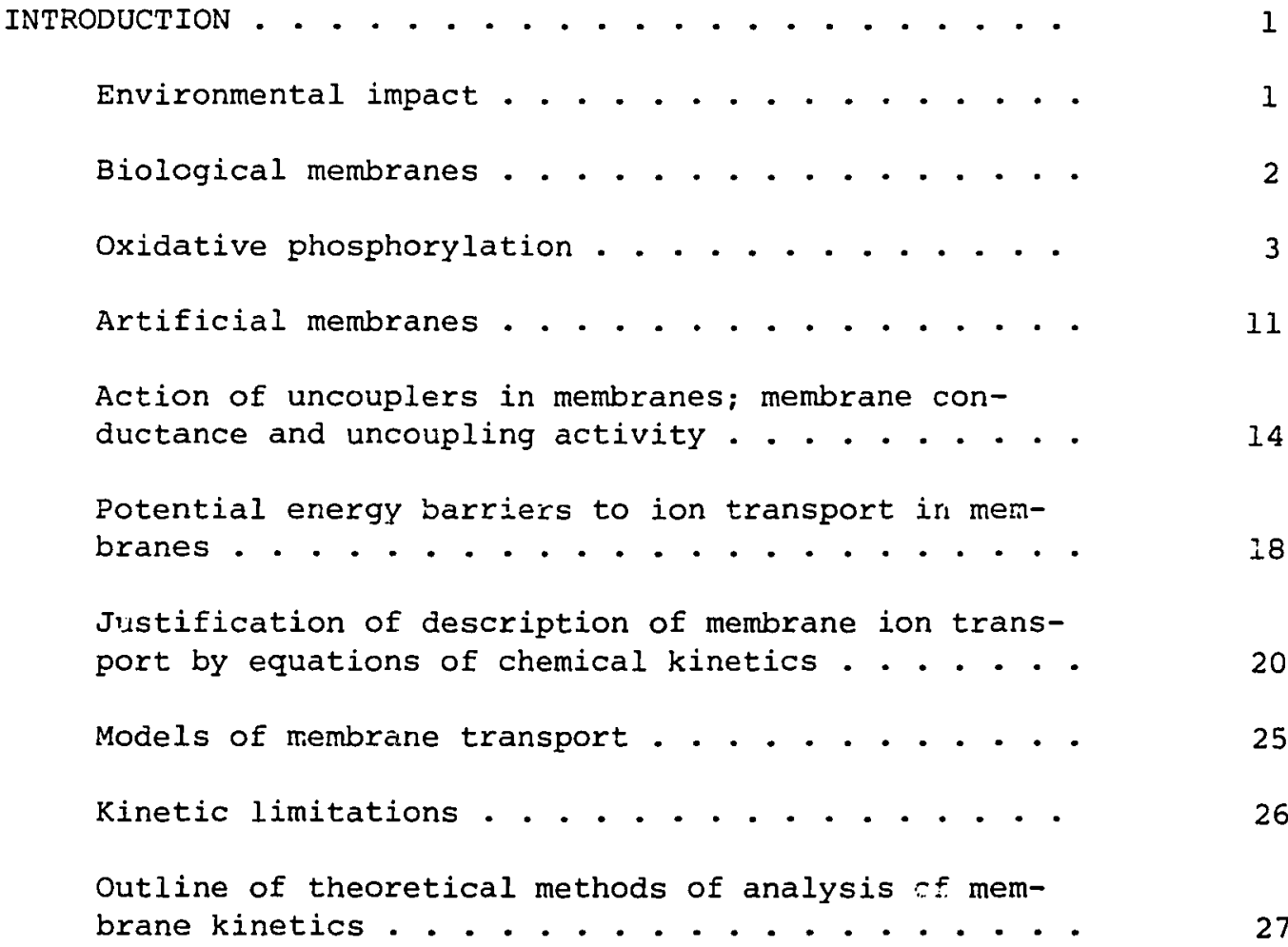

II METHODS AND MATERIALS . . . . . . . . . . . . . . .

Materials . . . . . . . . . . . . . . . . 
Membrane experiments . . . . . . . . . . . . 37

Current transient measurements . . . . . . . . . . 41

Microelectrophoresis experiments . . . . . . . . . . . 44

Algal toxicity experiments . . . . . . . . . . . . . 44

II I MEMBRANE TRANSPORT MODELS . . . . . . . . . . . . . . . . 47

Introduction . . . . . . . . . . . . . . . 4 47

Model 1: Relaxation due to ion redistribution in

membranes . . . . . . . . . . . . . . . . . 53

Model 2: Transmembrane translocation coupled to a

single surface reaction . . . . . . . . . . . . 56

Slow surface kinetics

Fast surface kinetics

Model 3: Transmembrane translocation coupled to two

identical surface reactions . . . . . . . . . . 63

Slow surface kinetics

Fast surface kinetics

Model 4: Transmembrane translocation coupled to two

interfacial transfer reactions . . . . . . . . . . . 70

A subsection of symmetric transport scheme

Slow interfacial kinetics

Fast interfacial kinetics

Model 5: General scheme for simple carrier mechanism. . .

System at steady state

Transient process

Charge translocation across the membrane as a

kinetically slow process

Fast return pathway

Fast surface reaction 
Model 6: Class I uncouplers . . . . . . . . . . . . 92 Properties of steady state flux

Transient flux parameters

Fast return pathway

Fast interface kinetics

Class II uncouplers: Introduction . . . . . . . . . . 99

Model 7: Dimer Model A . . . . . . . . . . . 101

Description of the model

Steady state properties

Kinetic limitations

slow return pathway

Slow interfacial process

Slow dimerization process

$\mathrm{pH}$ of conductivity maximum

Properties of transient flux

Model 8: Dimer model B . . . . . . . . . . . . . 114 Description of the model

Steady state properties

Kinetic limitations

$\mathrm{pH}$ of the conductivity maximum

Properties of transient flux 
Effect of buffer capacity . . . . . . . . . . . . . 152

Normalized membrane conductivity . . . . . . . . . . 155

Absence of kinetic limitations

Presence of kinetic limitations

Adsorption of pentachlorophenol anions to membranes . . . 164

Modification of $2,4,5-$ TCP induced membrane conduc-

tivity by tetraphenylarsonium and salicylate ions . . . . 168

Studies of Membrane Current Relaxation . . . . . . . . . 174

Toxicity of pertachlorophenol to alga . . ... . . . . 179

Main features of the mechanism of membrane conduc-

tion induced by $2,4,5-\mathrm{TCP}, \mathrm{PCP}$, and PCBT . . . . . . 183

Validity of small dimer density approximation . . . . . 188

Binding of PCP ions to phosphatidylcholine bilayers . . . 188

Existence of kinetic limitations . . . . . . . . . . . 190

Existence of a minimum in the net kinetic limita-

tion parameter at $\mathrm{pH}=\mathrm{pK}$. . . . . . . . . . . . 194

Modification of 2,4,5-TCP induced membrane conduc-

tivity by tetraphenylarsonium and salicylate ions . . . . . 196

Transient membrane currents . . . . . . . . . . . 202

Biological significance . . . . . . . . . . 206

VI

CONCLUSIONS . . . . . . . . . . . . . . . . . . 210

REFERENCES . . . . . . . . . . . . . . . . . . . . . . 212

APPENDIX: Reaction layer thickness . . . . . . . . . . . . . 219 


\section{LIST OF SYMBOLS}

A

$\mathrm{A}_{\mathrm{C}}$

$a_{H}$

$a_{\alpha j}$

${ }^{B} T$

${ }^{B} \mathrm{x}$

$\mathrm{CCCP}$

c

${ }_{\mathrm{C}}$

$\mathrm{c}_{\mathrm{T}}$

$c^{\prime}, c^{\prime \prime}$

$c^{1.4}$

D

$\mathrm{D}_{\mathrm{W}}$

DNP

DTEB

d

E

$\mathrm{E}_{\mathrm{e}}$
Anionic form of the uncoupler

Area of cross section of microelectrophoresis cell capillary tube

Activity of hydrogen ions

Coefficient of the time dependent terms in the variation with time of the density of components of membrane transport mechanisms

Total number of binding sites per unit area of membrane

Surface density of species $x$ bound to membranes

Carbonylcyanide m-chlorof':enylhydrazone

Concentration of ions in tile membrane (Eqs. 3, 5)

Aqueous concentration of species C

Total concentration of uncoupler in the aqueous phase

Concentration of membrane permeable ions at the left and right adsorption planes in the membrane (Eq. 7)

Radioactive isotope of carbon

Diffusion coefficient of membrane permeable ion in the membrane

Diffusion coefficient of membrane permeable ion in the water

2,4, -dinitrophenol

5,6-dichloro-2-trifluoromethylbenzimidazole

Thickness of the membrane

Energy associated with proton electrochemical gradient (Eq. 1)

Electric field on vesicles in microelectrophoresis cell capillary tube 


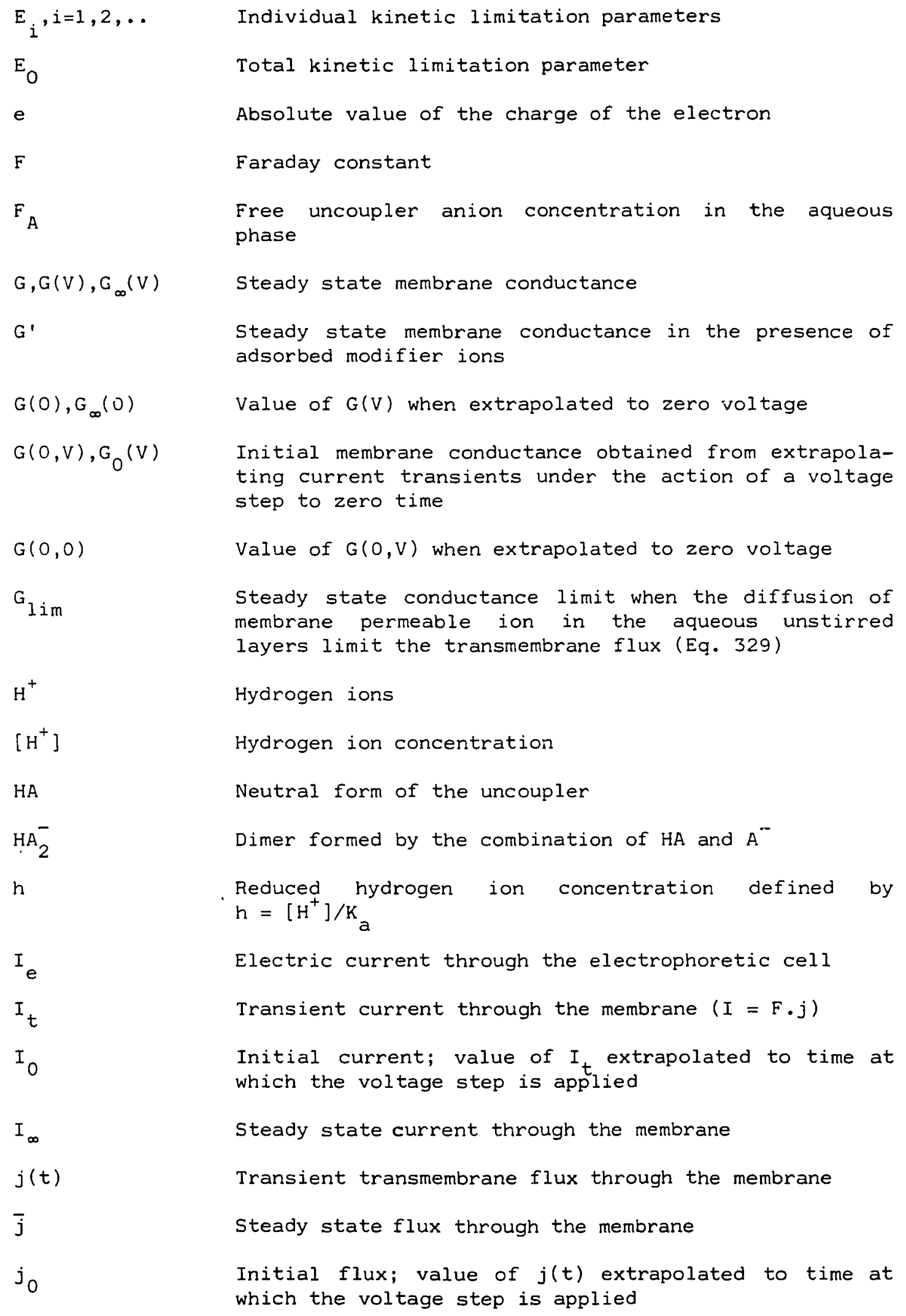




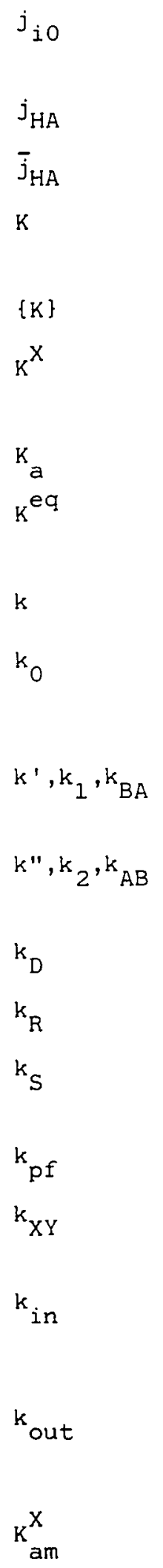

Flux amplitude of the component $i$ in the transient flux (Eqs. 75, 91)

Transient backward flux of HA across the membrane

Steady state backward flux of HA across the membrane

Electrical conductivity of vesicle solution used in microelectrophoresis experiments

Kinetic matrix (Eqs. 33, 206, 278)

Binding constant for adsorption of species $X$ to membranes (Eqs. 332, 347)

Dissociation constant of the uncoupler $\left(k_{a}=k_{D} / k_{R}\right)$

Equilibrium constant of formation of membrane permeable ion (model 3, Chapter III)

Boltzmann constant

Rate constant of translocation of membrane permeable ion across the membrane in the absence of electric field (Eq. 12)

Rate constant of translocation of membrane permeable ion across the membrane in forward direction (Eq. 13)

Rate constant of translocation of membrane permeable ion across the membrane in reverse direction (Eq. 14)

Rate constant of dissociation of uncoupler molecules

Rate constant of recombination of uncoupler molecules

Rate constant of backflow of neutral unsupler molecules

Rate constant defined by Eq. 43

Rate constant of transformation of $\mathrm{Y}+\mathrm{X}$ in kinetic models of membrane transport

Rate constant of transfer of species $C$ from the aqueous phase to the membrane phase in model 4 (Chapter III)

Rate constant of transfer of species $C$ from the membrane phase to the aqueous phase in model 4 (Chapter III)

Rate constant of transfer of species $x$ from the aqueous phase to the membrane phase 


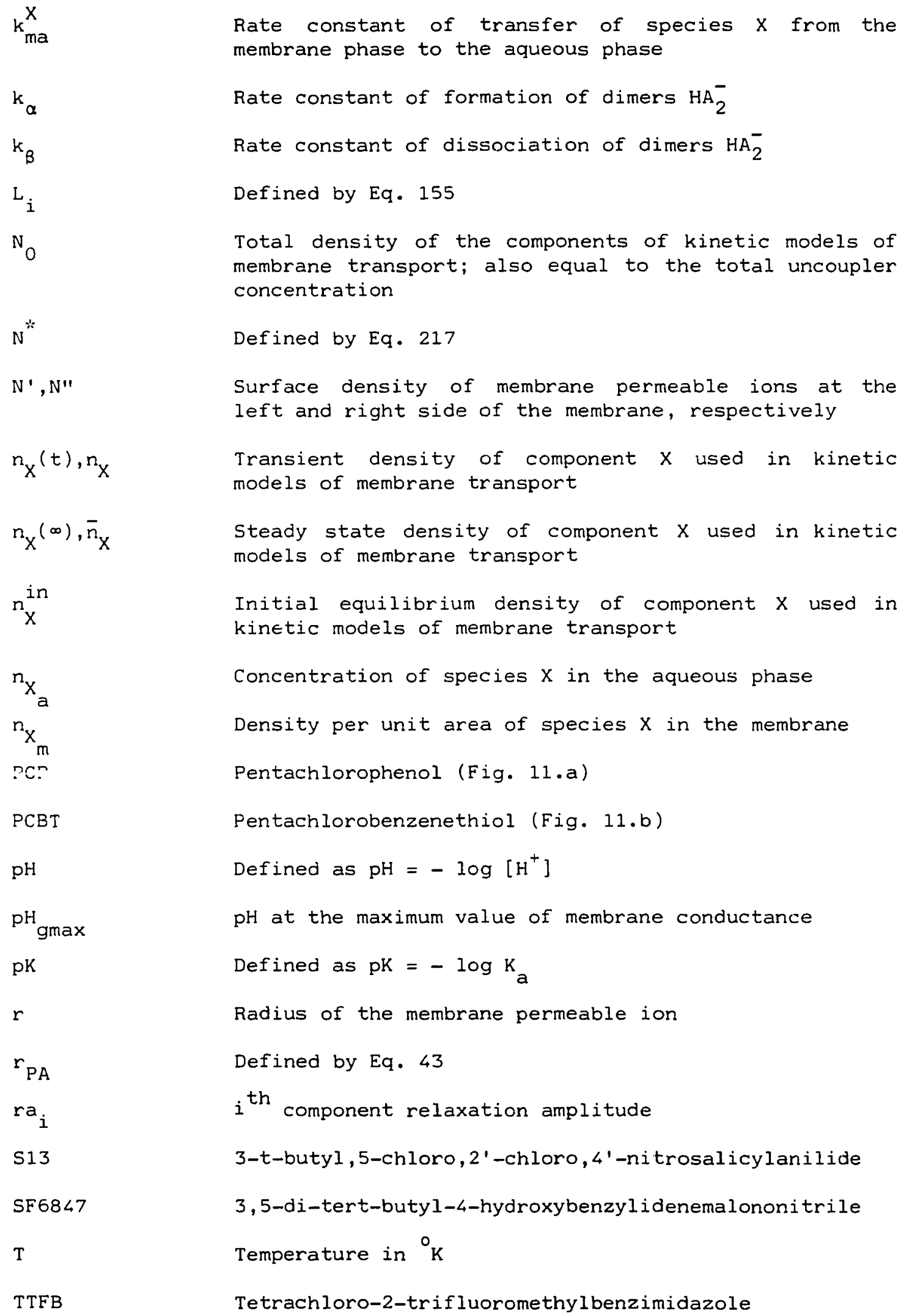$$
k_{m a}^{x}
$$$$
k_{\alpha}
$$$$
k_{B}
$$$$
\mathrm{L}_{i}
$$$$
\mathrm{N}_{\mathrm{O}}
$$$$
N^{*}
$$$$
N^{\prime}, N^{\prime \prime}
$$$$
n_{x}(t), n_{x}
$$$$
n_{x}(\infty), \bar{n}_{x}
$$$$
\mathrm{n}_{\mathrm{x}}^{\text {in }}
$$$$
\mathrm{n}_{\mathrm{a}}
$$$$
{ }^{n} \mathrm{~m}_{\mathrm{m}}
$$$$
\text { nce }
$$$$
\text { PCBT }
$$$$
\mathrm{pH}
$$$$
\mathrm{pH}_{\text {gmax }}
$$$$
\text { pk }
$$$$
r
$$$$
r_{P A}
$$$$
\mathrm{ra}_{\mathrm{i}}
$$$$
\text { S13 }
$$$$
\text { SF } 68<7
$$$$
\mathrm{T}
$$

Rate constant of transfer of species $x$ from the membrane phase to the aqueous phase

Rate constant of formation of dimers $\mathrm{HA}_{2}^{-}$

Rate constant of dissociation of dimers $\mathrm{HA}_{2}^{-}$

Defined by Eq. 155

Total density of the components of kinetic models of membrane transport; also equal to the total uncoupler concentration

Defined by Eq. 217

Surface density of membrane permeable ions at the left and right side of the membrane, respectively

Transient density of component $x$ used in kinetic models of membrane transport

Steady state density of component $x$ used in kinetic models of membrane transport

Initial equilibrium density of component $x$ used in kintic models of membrane transport

Concentration of species $X$ in the aqueous phase

Density per unit area of species $X$ in the membrane

Pentachlorophenol (Fig. 11.a)

Pentachlorobenzenethiol (Fig. 11.b)

Defined as $\mathrm{pH}=-\log \left[\mathrm{H}^{+}\right]$

$\mathrm{pH}$ at the maximum value of membrane conductance

Defined as $\mathrm{pK}=-\log \mathrm{K}_{\mathrm{a}}$

Radius of the membrane permeable ion

Defined by Eq. 43

$i^{\text {th }}$ component relaxation amplitude

3-t-butyl ,5-chloro, $2^{\prime}$-chloro, 4'-nitrosalicylanilide

3,5-di-tert-butyl-4-hydroxybenzylidenemalononitrile

Temperature in ${ }^{\circ} \mathrm{K}$

Tetrachloro-2-trifluoromethylbenzimidazole 
$t$

u

V

$\mathrm{V}_{\mathrm{e}}$

$w_{0}$

$w_{b}$

$W(x)$

$w(n)$

$w_{1}(x)$

$X_{A}$

z

$2,4,5-\mathrm{TCP}$

',"

$\alpha$

B

$\gamma$

$\gamma_{\omega}$

$\delta$

$\varepsilon_{\mathrm{m}}$

$\varepsilon_{\omega}$

$\eta$

$\lambda$

$\mu$

$\mu_{e}$
Time

Mobility of ions in the membrane (Eq. 3)

Potential difference applied across the membrane

Electrophoretic velocity of vesicles

Born charging energy; energy required to move an ion from the aqueous phase to the membrane phase (Eq. 2)

Membrane barrier height

Image charge membrane potential energy barrier

Hydrophobic energy potential energy well

Defined as equal to $w(x)-W(n)$

Density displacement of species A from initial equilibrium value $\left(x_{A}=n_{A}^{I n}-n_{A}\right)$

Valency of the ion

2,4,5-trichlorophenol (Fig. 11.c)

Primes and double primes denote species at the left and right hand side of the membrane, respectively

Relaxation amplitude defined by Eq. 50

Fraction of the externally applied voltage experienced by the membrane permeable ion $=(d-2 . \pi) / d$

Reaction layer thickness (see appendix)

Width of ion adsorption well in the membrane

Thickness of unstirred layer

Dielectric constant of membrane

Dielectric constant of water

Depth of ion adsorption layer from the membrane surface

Reciprocal of relaxation time constant $(\lambda=1 / \tau)$

Electrochemical potential (Eq. 4)

Electrophoretic mobility 
$\sigma_{i}$

$\tau$

zeф

$\Psi$ m

$\Psi$ S

$z e \Psi$

$\omega$

Defined by Eqs. 213-216 in dimer model A (Chapter III), and by Eqs. 286 - 289 in dimer model B (Chapter III)

Relaxation time constant

Electric potential energy of an ion in the membrane

Membrane potential induced by adsorbed modifier ions and determined from Eq. 346

Potential at hydrodynamic plane of shear of vesicles (zeta potential)

Total potential energy of an ion in the membrane

Defined in Eq. 8 


\section{LIST OF TABLES}

I Modified standard AAP-EPA medium for alga . . . . . . . 45

II Dependence of various types of kinetic limitations in dimer models $\mathrm{A}$ and $\mathrm{B}$ on the aqueous $\mathrm{pH}$ and the uncoupler concentration. . . . . . . . . . . . . 135 
LIST OF FIGURES

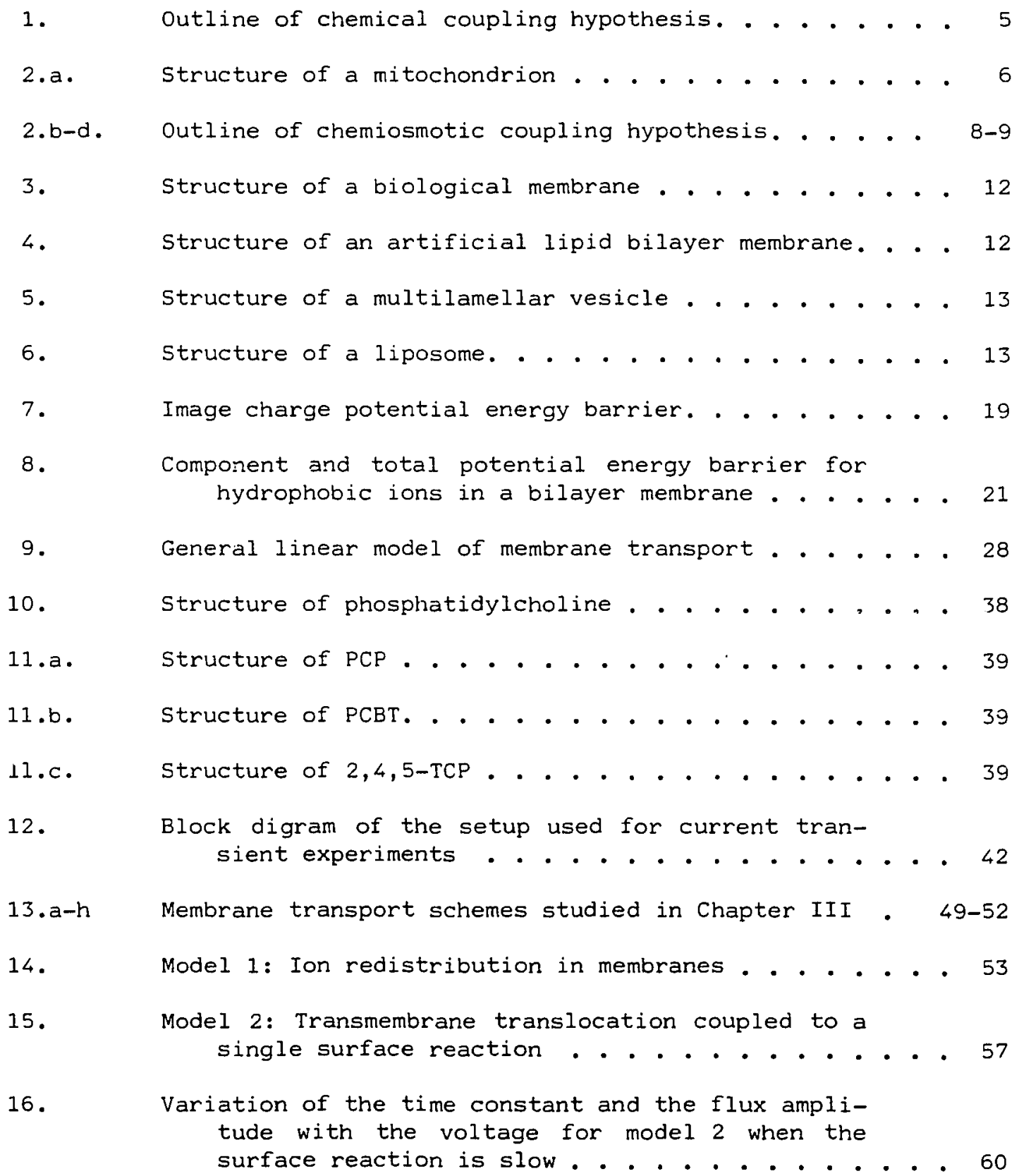


17. Variation of the time constant and the flux amplitude with the voltage for model 2 when the surface reaction is fast. . . . . . . . . . .

18. Model 3: Transmembrane translocation coupled to two identical surface reactions . . . . . . . 63

19. Variation of the time constant and the flux ampli-

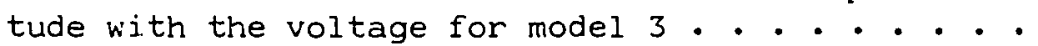

20. Model 4: Transmembrane translocation coupled to two interfacial transfer reactions. . . . . . . .

Variation of the normalized membrane conductance with the voltage for various values of the kinetic limitation parameter. . . . . . . . . .

Variation of the relaxation amplitude with the voltage for various values of the kinetic limitation parameter. . . . . . . . . . . . .

23. Variation of the relaxation time constant with the voltage for various values of the kinetic limitation parameter. . . . . . . . . . .

24. Model 5: General scheme for simple carrier mechanisms. . . . . . . . . . . . . . . . . .

25. Effect of the kinetic Iimitation parameter on the normalized membrane conductance curves. . . . . .

26.

Relationship between the relative displacement of the density of species from the initial equilibrium values to the final steady state val-

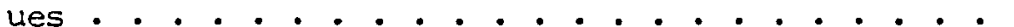

27.

Variation of the relaxation characteristics with the voltage for model 5 when the surface reaction is slow and the rate of backflow is fast. . . . . . . . . . . . . . . . .

28.

Variation of the relaxation characteristics with the voltage for model 5 when the surface reaction is fast and the rate of backflow is slow. . . . . . . . . . . . . . . . . . .

29.

Transition from one kinetic regime to another with the increase of the voltage observed in the relaxation characteristics of model $5 . .$. . 
Variation of the relaxation characteristics with the voltage and the $\mathrm{pH}$ for model 6 when the rate of return flow of HA is fast. . . . . . . 98

Variation of relaxation characteristics with voltage and $\mathrm{pH}$ for model 6 when the rate of interfacial reaction is fast . . . . . . . 100

Typical curves for the variation of the relaxation characteristics with the voltage for the dimer model A. . . . . . . . . . . . . .

Variation of the zero voltage time constant and the relaxation amplitude with the $\mathrm{pH}$ when the rate limiting step is either the backflow of uncouplers, or the proton association-dissociation reaction . . . . . . . . . . . . .

36.

Variation of the zero voltage time constant and the relaxation amplitude with the uncoupler concentration when the rate limiting step is either the backflow of neutral uncouplers or the proton association-dissociation reaction . . 119

37. Graph showing the linear relationship between the relaxation ainplitude and the uncoupler concentration............... . . 120

38. Model 8: Transport scheme for the dimer model B * • . 121

39. Variation of the relaxation characteristics with $\mathrm{pH}$ for dimer model $\mathrm{B}$ at various kinetic limitations... . . . . . . . . . . . . 139

40. Variation of the relaxation characteristics with the uncoupler concentration for the dimer model B at various kinetic limitations . . . . . . 141

41 Graph showing the Iinear relationship between the relaxation amplitude and the uncoupler concen-

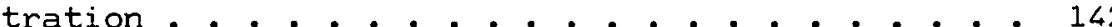

42. $\mathrm{pH}$ dependence of the membrane conductance and the uncoupling activity induced by PCBT . . . . . . . 144 
xviii

43. $\mathrm{pH}$ dependence of the PCP induced merbrane conductance and the density of PCP ions adsorbed to vesicles . . . . . . . . . . . . . . 146

44. $\mathrm{pH}$ dependence of the 2,4,5-TCP induced membrane conductance . . . . . . . . . . . . . .

45. Dependence of the membrane conductance on the PCBT concentration . . . . . . . . . . . 149

46. Dependence of the membrane conductance on the PCP concentration. . . . . . . . . . . . 150

47. Dependence of the membrane conductance on the $2,4,5-\mathrm{TCP}$ concentration . . . . . . . . . . 151

48. Variation of the PCP induced membrane potential per unit of transmembrane $\mathrm{pH}$ difference, as a function of the $\mathrm{pH}$ of the aqueous phase. . . . .

49. Effect of the buffer concentration on the PCBT induced membrane conductance . . . . . . . . . 154

50. Linear dependence of the PCBT induced slow transient current on reciprocal square root of time observed at low buffer concentration. . . .

51. Variation of the 2,4,5-TCP induced normalized membrane conductance with the voltage in the absence of kinetic limitations . . . . . . . .

Variation of the PCP induced normalized membrane conductance with the voltage . . . . . . . . .

53.

Variation of the PCBT induced normalized initial membrane conductance, obtained from current relaxation data, with the voltage. . . . . . .

54. Voltage dependence of the normalized membrane conductance at high concentration of 2,4,5-TCP, indicating the development of kinetic limita-

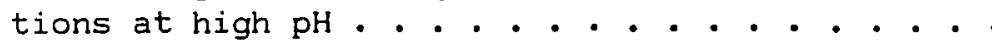

55. Voltage dependence of the PCBT induced normalized membrane conductance, indicating the development of kinetic limitations at high and low $\mathrm{pH}$. .

56 .

Voltage dependence of the PCBT induced normalized membrane conductance, indicating the development of kinetic limitations at increasing concentration of $\mathrm{PCBT}$. . . . . . . . . . . . . . 
57.

58.

59.

60.

60.

61.

62.

\section{3.}

64.

65.

66.

67.

68.

69. Variation of the electrophoretic mobility and the
corresponding electric potential (zeta poten-
tial) with the aqueous PCP concentration. . . . . 166

Variation of the density of PCP anions adsorbed to the membrane of vesicles, with the aqueous concentration of PCP anions . . . . . . . . . 167

Scatchard plot for the adsorption of PCP anions to membranes. . . . . . . . . . . . . 169

Enhancement due to tetraphenylarsonium and suppression due to salicylate ions of the 2,4,5TCP induced membrane conductance with increasing concentration of adsorbed modifier ions . . . . 170

Variation of the membrane potential induced by the adsorbed tetraphenylarsonium and salicylate ions with increasing concentration of modifier ions . . . . . . . . . . . . . .

$\mathrm{pH}$ dependence of the 2,4,5-TCP induced membrane conductance in the presence of tetraphenylarsonium ions, salicylate ions, and in their absence . . . . . . . . . . . . . . 173

Variation of the PCBT induced relaxation characteristics with the voltage. . . . . . . . . 175

Variation of tine time constant with the $\mathrm{pH}$ for PCP induced current relaxations . . . . . . . . 176

Variation of the time constant with the uncoupler concentration for $2,4,5-\mathrm{TCP}$ induced current relaxations . . . . . . . . . . . . . 178

Variation of the rate of $\mathrm{C}^{14}$ uptake by alga with increasing concentration of PCP . . . . . . . . 180

Characteristic $\mathrm{pH}$ dependence of the rate of carbon uptake by alga in the absence of uncoupler . . 182

Potential energy profile in the membrane and in the adjacent aqueous layers due to uniformly distributed negative surface charge alone . . . . 197

9. Potential energy profile in the membrane and in the adjacent aqueous layers due to uniformly distributed positive surface charge alone . . . . 197 
70.

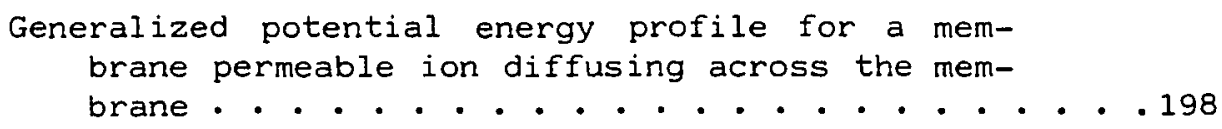

71. Interfacial processes occurring in dimer model B at the positively biased membrane surface . . . . . 220 
CHAPTER I

INTRODUCTION

ENVIRONMENTAL IMPACT

The compounds of interest are chlorinated phenols. They have many technological and economical applications as pesticides and bactericides. These compounds share many of the beneficial as well as negative characteristics with other organochlorines. Their environmental ill effects are a result of the following factors.

(a) Nonspecificity: There is no pesticide which will selectively destroy only one species of pests. They are equally poisonous to other "non harmful" or "helpful" organisms.

(b) Resistance: Sometimes the target organism develops a resistance to the pesticide. When combined with nonspecificity of the pesticide the development of resistance could in fact lead to effects opposite to that intended.

(c) Persistence: Most naturally occurring organic compounds are biodegradable; synthetic chemicals like organochlorines persist in the environment for a long time. Sometimes their decomposition products are also poisonous.

(d) Biological magnification: Because of high solubility of pesticides in nonpolar solvents they are accumulated in the fatty tissues of animals and primary producers such as alga, which are at the origin of the food chain. The accumulation of pesticides in the primary consumers which feed on alga results in the accumulation and in higher pesticide concentrations higher in the food web. This gives rise to the pnenome- 
nor of biological magnification. Since man lies at the highest point in the food web the potential for the accumulation of pesticides in man is high.

It is known that all living things including man carry at least trace amounts of pesticide in their tissues. Therefore it is important to understand the effect of pesticides on biological systems, especially on membranes, where the majority of biological functions, including energy production, occur.

BIOLOGICAL MEMBRANES

\begin{abstract}
Biomembranes are the sites of numerous processes important for the existence of life. They maintain and regulate the effects of the environment surrounding them by distributing solutes and they are the sites of the energy conversion processes. Some of the functions of biomembranes are as follows.
\end{abstract}

(a) Compartmentalization: There are many membrane bound cells and organelles in which the membrane acts as a permeability barrier to separating the biomaterial inside from that of the outside compartment. This separation provides the means for ensuring high local concentrations of substrates by enclosing solutes in vesicles or organelles. It also provides for the bulk-transfer of material by the membrane engulfment of liquid droplets (pinocytosis) or of solids (phagocytosis). Transport across membranes or other membrane involving processes are universal properties of all cells.

(b) Transport: The transport of ions and molecules through biomembranes can be catagorized into two classes, active and passive. Passive transport is a dissipative process and does not require a 
direct supply of energy and results in the lowering of the electrochemical gradient of solutes existing across the membrane. Active transport utilizes energy from various sources and is usually associated with movement of ions and molecules across membranes from regions of low concentration to regions of high concentration. In this dissertation we are concerned with the passive transport of protons across the energy transducing membranes of mitochondria, bacteria, and chloroplasts by chlorinated phenols.

Action of chlorinated phenols results in the dissipation of the electrochemical gradient of hydrogen ions across the membranes of these organelles. This dissipation is associated with their primary toxic effect.

The transport of ions and molecules through membranes is mediated by membrane pores, channels, or by membrane permeable carrier-solute complexes.

A transmembrane channel is usually formed by the association of several macromolecules to form an opening in the membrane and it has specific binding sites inside. A pore is usually a wide channel, nonselective to small solutes, which does not have specific binding sites.

A carrier is one or several molecules which form a membrane permeable complex; the ion or molecule is transferred by diffusion of the complex across the membrane. A proton transporting carrier is called a protonophore.

OXIDATIVE PHOSPHORYLATION

The energy needed for various metabolic processes is often ob- 
tained from the release of phosphate by adenosine triphosphate (ATP). The reverse process, production of ATP, taking place in the presence of oxygen is called oxidative phosphorylation and occurs at the inner membrane of mitochondria. Most of the macroscopic details of this process have been worked out. Briefly, oxidation of foodstuff ultimately results in the flow of electrons and protons through a series of protein molecules located in the energy transducing membrane and known as the electron transport chain. The energy derived from this process is used to convert adenosine diphosphate (ADP) to energy rich adenosine triphosphate (ATP), according to the scheme

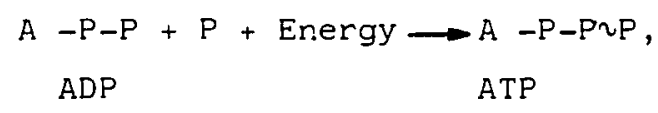

where A represents the adenosine group, $P$ the phosphate group, and the symbol $\sim$ is used to indicate that this bond can be broken with the release of energy. Although there is general understanding of the energy conservation process, there is still a controversy about the mechanism of the coupling of the electron transport to the phosphorylation of ADP to ATP. Chlorinated phenols have the ability to uncouple oxidative phosphorylation from the electron transport. Thus, our understanding of the effect of chlorinated phenols at the membrane lovel is useful for and should be consistent with the concept of coupling.

Three major hypotheses of coupling have heen put forward.

(a) Chemical coupling hypothesis. According to the chemical coupling hypothesis electron transport is coupled to phosphorylation through a common high energy chemical intermediate. This hypothesis requires the presence of two components $X$ and $I$ in addition to consti- 
tuents of the electron transport chain. Energy is conserved by the formation of a common high energy intermediate $X^{2} I$ with an energy $r i c h$ bond, which is then broken and energy released to the ATP molecule. This process is outlined in Fig. 1. However, after twenty years of

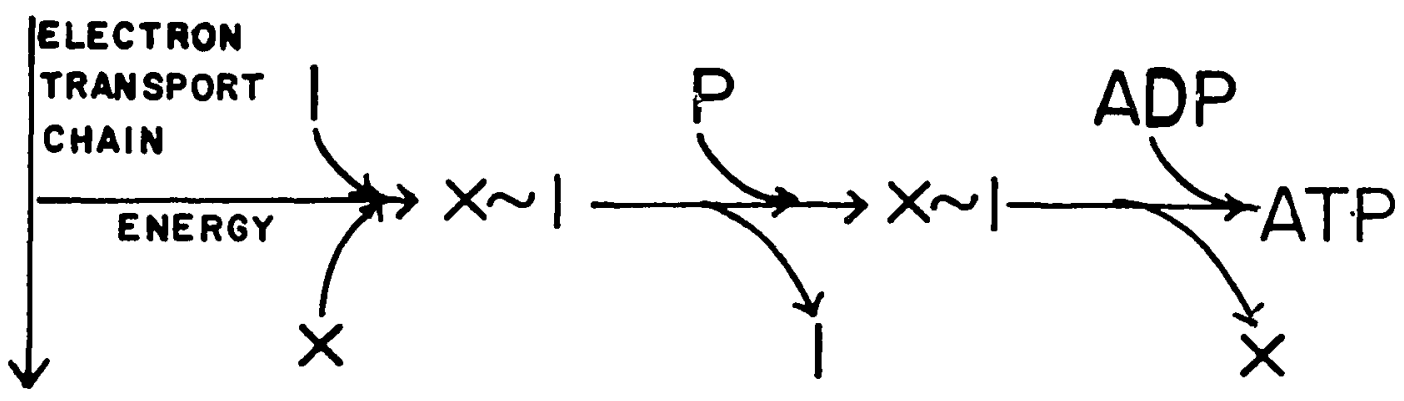

Figure 1. Outline of chemical coupling hypothesis.

intensive research the high energy intermediate has not been detected. Many feel that such an intermediate does not exist. Another difficulty with the chemical coupling hypothesis is that it does not provide a satisfactory explanation for the fact that a completely intact and continuous inner mitrochondrial membrane is a necessary requirement for oxidative phosphorylation. In fact, membranes are not required for chemical coupling reactions. One of the arguments for the existence of these intermediates in membranes is that the membrane provides a non-polar environment necessary for formation of intermediates.

(b) Conformational coupling hypothesis. According to the conformational coupling hypothesis (1) the energy derived from electron transport is conserved in the form of a conformational change in an electron-carrier-protein or the enzyme $\mathrm{F}_{1}$-ATPase. The high energy conformational state is assumed to drive the ATP synthesis in the process of the transition of the protein into the low energy conformational 
state. The conformational coupling hypothesis is in principle a variation of the chemical coupling hypothesis. In the chemical coupling hypothesis the energy is stored in a high energy covalent bond whereas in the conformational hypothesis the high energy intermediate state can be associated with a number of weak non-covalent bonds.

(c) Chemiosmotic coupling hypothesis. According to chemiosmotic coupling hypothesis $(2,3)$ there are no chemical compounds coupling electron transport to phosphorylation. Instead, the energy is conserved through the development of a proton electrochemical gradient across the inner mitochondrial membrane (Fig. 2.a). The series of chemical reac-

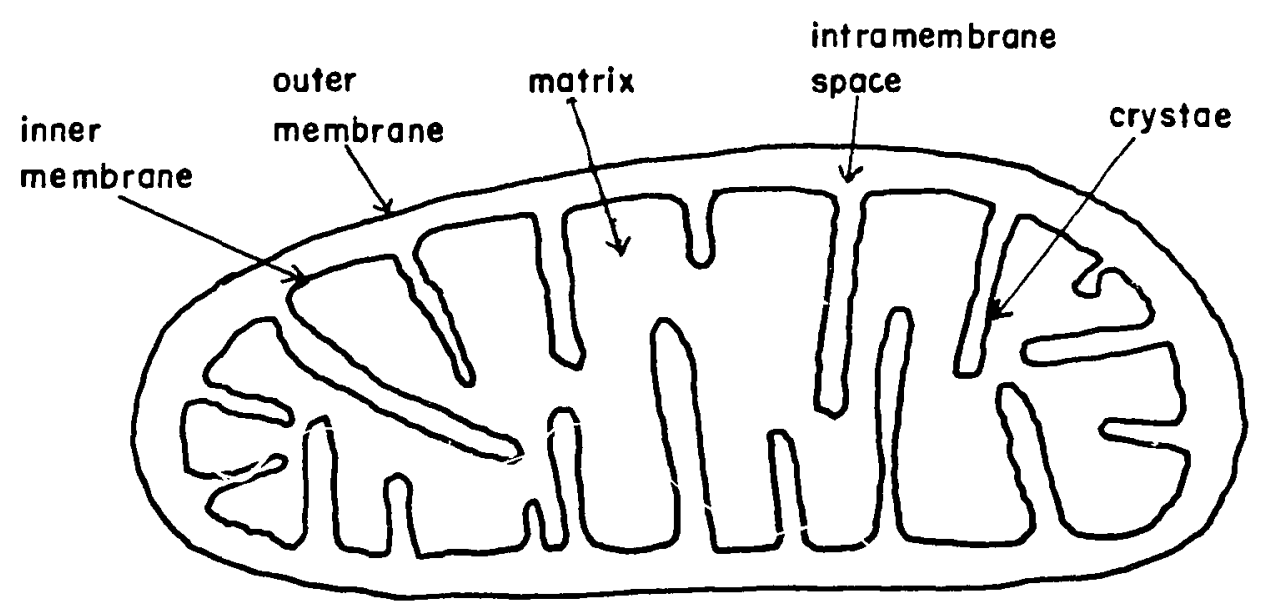

Figure 2.a. Structure of a motochondrion. 
tions taking place along with the transport of electrons through the system of electron acceptors and electron donors in the inner mitochondrial membrane results in pumping of protons from the inside to the outside of inner mitochondrial space as shown schematically in Fig. 2.b. Ultimately, the electron is used to convert oxygen into water. The resulting transmembrane electrochemical gradient of hydrogen ions, E, (Eq. 1) (Fig. 2.C) has two components; one is the chemical potential energy of the proton concentration gradient (first term in the Eq. 1), and the other the electric potential energy associated with the electric potential difference across the membrane (second term in the Eq. 1). The total energy $E$ is given by

$$
E=k T \cdot \ln \frac{\left[\mathrm{H}^{+}\right]_{\text {out }}}{\left[\mathrm{H}^{+}\right]_{\text {in }}}+\mathrm{e} \cdot\left(\Psi_{\text {out }}-\Psi_{\text {in }}\right) \text {, }
$$

where $\left[\mathrm{H}^{+}\right]$denotes the proton concentration, and $\Psi$ the electric potential; "in" and "out" refer to the matrix and intramembrane space of the mitochondrion. The proton electrochemical gradient drives the ATP synthesis according to the reaction shown in Fig. 2.d. The $\mathrm{H}^{+}$formed in this reaction moves spontaneously to the side with the high concentration of $\mathrm{OH}^{-}$, which is inside the mitochondrion (matrix). The $\mathrm{OH}^{-}$formed moves spontaneously to the outside mitochondrial space (intramembrane space) where the $\mathrm{H}^{+}$concentration is high. The above reaction takes place in the enzyme $F_{1}$-ATPase. The processes occurring in photosynthetic phosphorylation are very similar, except that the primary energy is derived from photons only.

Oxidative phosphorylation is influenced by a number of chemical agents which fall into two major classes; uncouplers and inhibitors. 
intramembrone

space
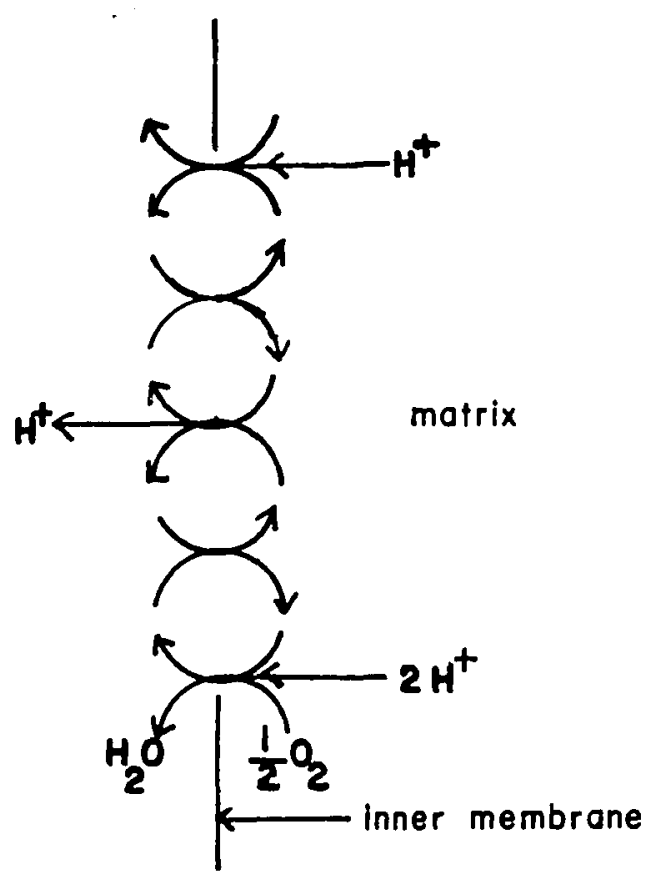

Figure 2.b. Transport of electrons through the electron transport chain in the inner membrane of mitochondria results in pumping of protons from the matrix to the intramembrane space.

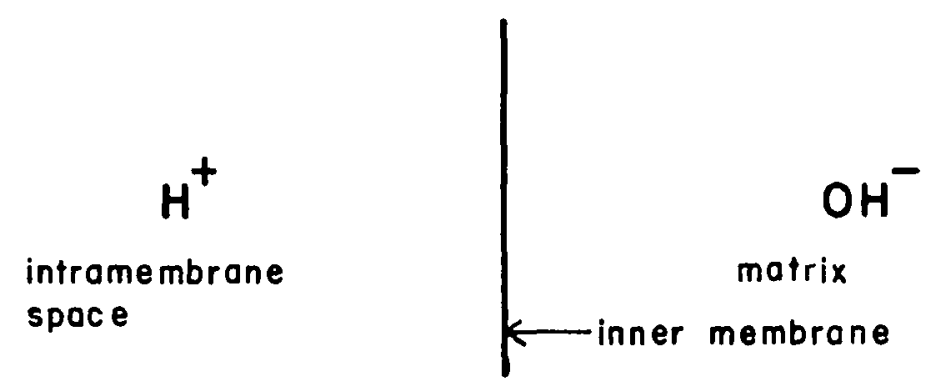

Figure 2.c. The pumping of protons results in the development of a proton electrochemical gradient across the inner mitochondrial membrane. 

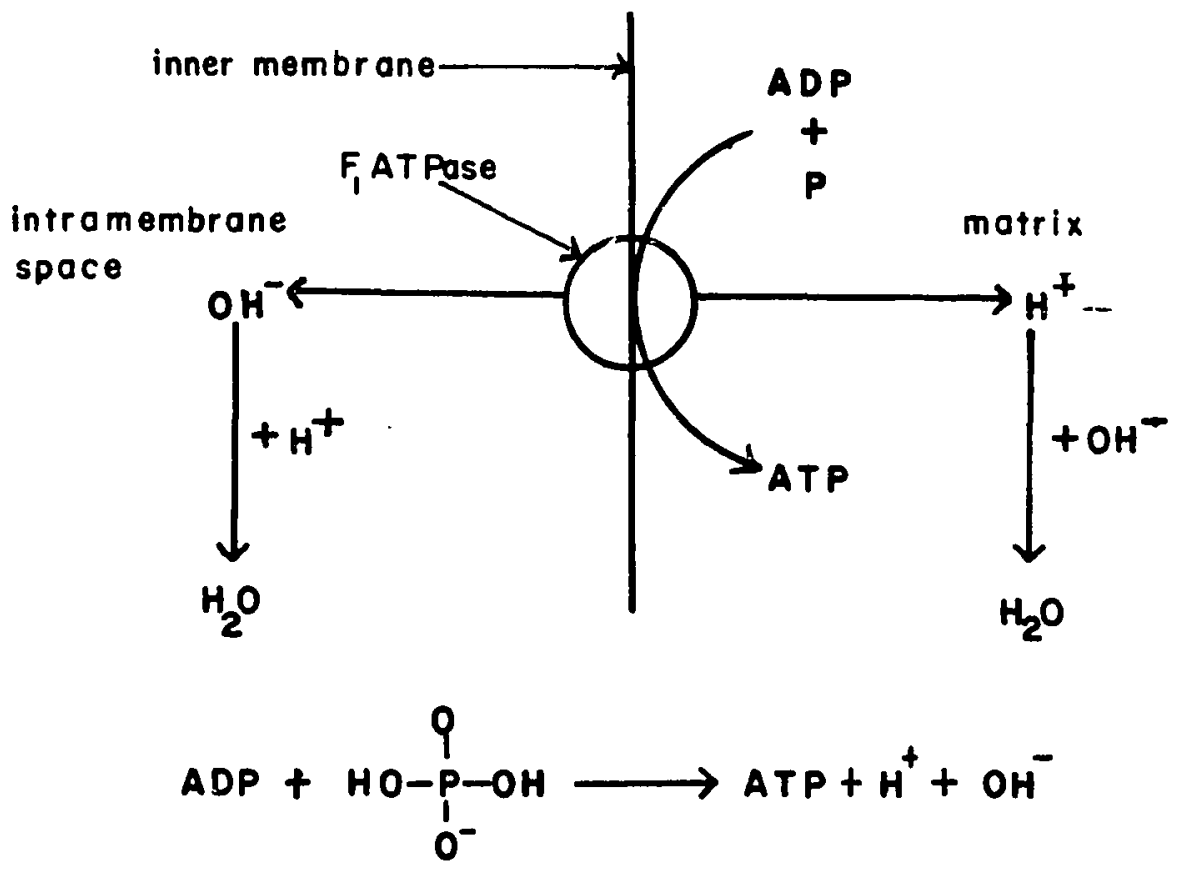

Figure 2.d. According to chemiosmotic coupling hypothesis the energy of the proton electrochemical gradient drives the ATP synthesis.

Uncouplers prevent the formation of ATP without disrupting the electron transport, whereas inhibitors prevent both the electron transport and ATP synthesis.

Two classes of weak acid uncouplers are recognized, distinguished by their action in lipid membranes. Class I uncouplers induce conductance in lipid bilayer membranes that is proportional to uncoupler concentration. Class II uncouplers induce conductance in lipid bilayer membranes that depend quadratically on uncoupler concentration. Examples of class I uncouplers are SF6847(4), carbonylcyanide m-chlorophenylhydrazone (CCCP) (5), and 3-t-butyl,5-chloro,2'-chloro, 4'-nitrosalicylanilide (S13) (6). Examples of class II uncouplers are 2,4-dini- 
trophenol (DNP) (7), 5,6-dichloro-2-trifluoromethylbenzimidazole (DTFB) (8), and tetrachloro-2-trifluoromethylbenzimidazole (TTFB) (9). An example of an inhibitor is oligomycin (10). According to the chemical and conformational. coupling hypotheses the uncouplers either break down or discharge the high energy intermediate thus preventing ATP formation. According to the chemiosmotic hypothesis uncouplers dissipate the energy of the proton electrochemical gradient existing across the inner mitochondrial membrane. Ionophores and protonophores can act as uncouplers by reducing the electrochemical potential difference of hydrogen ions that exist across the inner mitochondrial membrane or across the thylakoid membrane in chloroplasts. In either case the reduced proton electrochemical gradient becomes insufficient for the production of ATP.

The validity of the coupling hypotheses has been tested using artificial membrane systems. According to the chemiosmotic hypothesis, the toxic action of weak acids consists in the uncoupling of phosphorylation from electron transport, by their protonophoretic ability (11). Therefore they are expected to and in fact they do transport protons across all lipid membranes. The rate of proton translocation induced by uncouplers should correlate with their uncoupling activity.

So far all uncouplers have been found to induce electrical conductivity in artificial bilayer membranes and the conductivity is associated with translocation of protons $(8)$. We will return to the problem of the correlation between uncoupling activity and electrical conductance induced in bilayer membranes after the review of properties of artificial membrane systems. 
ARTIFICIAL MEMBRANES

At the present stage in the development of membrane biophysics, biological membranes appear to be too complex for simple analysis. Their complexity is due to: (1) the many lipid structures constituting the membrane, (2) the non-uniform distribution of lipids within the membrane, and (3) the presence of proveins (12).

It is advantageous to use artificial lipid membranes in the detailed studies of many transport mechanisms, and for the testing of the different hypotheses relating membrane structure and membrane properties. Artificial membranes have simpler and better defined structure and their properties are reproducible (13). Many artificial membrane transport systems have been well characterized (5,9). Studies on artificial membranes are biologically relevant because the membranes are prepared from the lipids occurring in natural biomembranes.

The existence of polar head groups and hydrocarbon chains in the lipid molecule (Fig. 10) is essential to the existence of lipid bilayer membrane (Figs. 3-6) (14). Membranes are dynamic structures (15); the lipids readily undergo lateral diffusion along their plane $\left(D=10^{-8}\right.$ $\left.-10^{-10} \mathrm{~cm}^{2} / \mathrm{s}\right)(16)$, and much less readily across the membrane (flipflop) (17-19). The primary distinction between the biological membranes (20) and the artificial membranes (21) is depicted in Figs. 3 and 4. Lipids mixed in water in a flask spontaneously form multilamellar vesicles having structures such as shown in Fig. 5 (22). These vesicles are frequently used in adsorption studies (23). It has furthermore been shown possible to reconstitute a number of essential life supporting systems $(24,25)$ including oxidative phosphorylation (26) in single layered vesicles (27) (Fig. 6). 


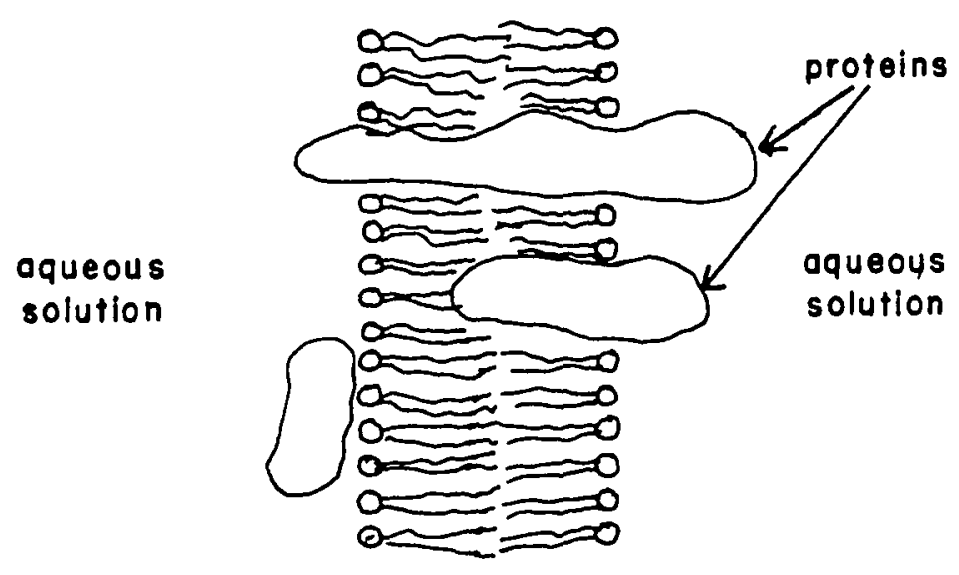

Figure 3. Structure of a biological membrane.

oqueous

solution

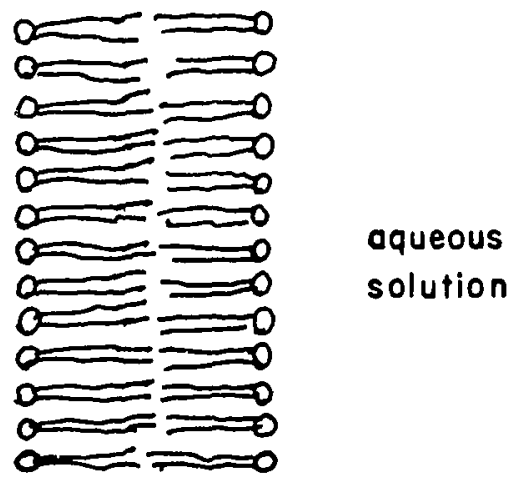

Figure 4. Structure of an artificial lipid bilayer membrane. 


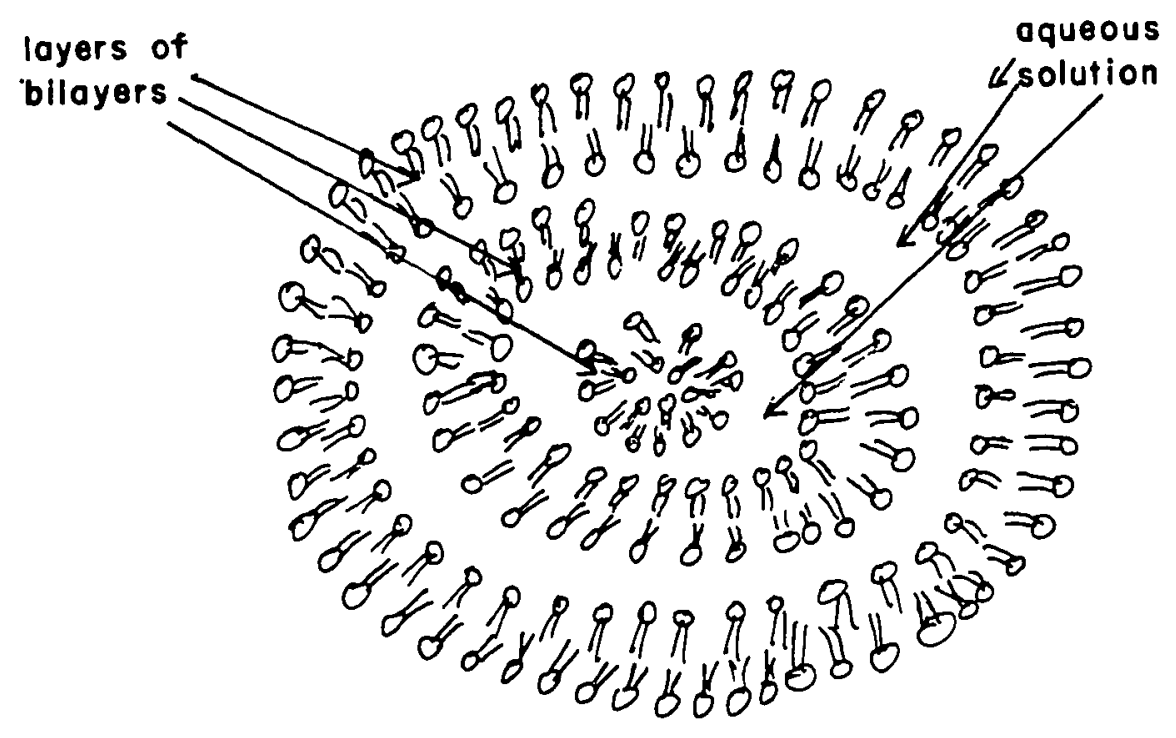

Figure 5. Structure of a multilamellar vesicle.

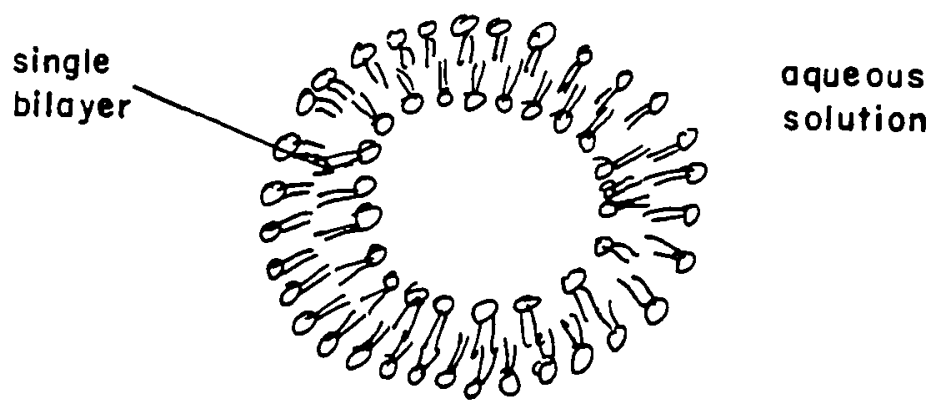

Figure 6. Structure of a liposome. 
Planar bilayer membranes are useful tools for electric field driven studies of membrane ion transport. We have used them in this project to advance our understanding of action of chlorinated phenols on memiranes at the molecular level. We have been particularly interested in the relationship between the effect of induced electrical conductivity and the alteration of membrane biological function.

\title{
ACTION OF UNCOUPLERS IN MEMBRANES; \\ MEMBRANE CONDUCTANCE AND UNCOUPLING ACTIVITY
}

\begin{abstract}
Studies of membrane conductivity induced by weak acid uncouplers indicate that transmembrane charge transport is kinetically linked to the translocation of protons across 1 ipid membranes $(5,7,8,28-30)$. This result is in general agreement with the chemiosmotic hypothesis according to which the biological action of weak acids, and the uncoupling of oxidative and photosynthetic phosphorylation, is associated with the movement of protons across biological membranes (2). Thus if the chemiosmotic hypothesis is correct, one would expect to find that (a) all weak acid uncouplers induce conductivity in lipid bilayer membranes, (b) all weak acids that increase the conductivity of artificial lipid membranes also uncouple phosphorylation, and (c) there is a correlation between the magnitude of membrane conductance and uncoupling activity. Many weak acid uncouplers have been found to increase electrical conductance of lipid membranes (5,7-9,28-30). However, there have been reports of discrepancies between the above mentioned expectations and the experimental findings (31-34). In recent studies some of the discrepancies have been resolved (35). This project represents a contribution to these efforts.
\end{abstract}


It was reported that one of the exceptional weak acid uncouplers was thiosalicylate since it was found to be biologically active but did not influence the conductivity of lipid membranes $(31,32)$. This observation contradicted chemiosmotic concept and also the observation that thiosalicylate increased the flow of hydrogen ions in lipid vesicles (31). It has been shown that the failure to observe thiosalicylate induced membrane conductivity was due to "leaky" membranes which had high background conductance (35). It was found necessary to prepare membranes from highly purified lipids in order to demonstrate the increase of membrane conductivity in the presence of thiosalicylate (35).

The validity of chemiosmotic hypothesis would also be questionable if there were weak acids which increased the conductivity of lipid membranes but would not act as uncouplers in biological systems. Picrate was reported to be such a compound $(36,37)$. Picrate did not uncouple mitochondria, but it was found to uncouple submitochondrial particles (36). This observation prompted some investigators (33) to conclude that picrate is completely impermeable in inner mitochondrial membrane, and to suggest that uncoupling activity of picrate, and perhaps of all weak acid uncouplers, is due to their binding to a specific site at the interior surface of the inner mitochondrial membrane. Binding of uncouplers to specific sites was supposed to discharge the high energy intermediate postulated in chemical and conformational coupling hypotheses. The above interpretation of action of picrate seemed to contradict chemiosmotic hypothesis and favor applicability of alternate concepts of coupling. McLaughlin et al. (35) realized the significance of the fact that vesicles prepared from 
mitochondrial membranes are "inverted", i.e. the interior membrane of mitochondria becomes the exterior membrane of submitochondrial vesicles, which results in the reversal of electric field in the transducing membranes. These authors presented alternative quantitative explanations for the unusual experimental results obtained with picrate. These results are now in agreement with the chemiosmotic hypothesis.

The reason for the exceptional property of picrate rests on the fact that its $\mathrm{pK}_{a}$ is low $(=0.3)$ and the anionic form is highly membrane permeable (37). Thus at physiological $\mathrm{pH}$ essentially all picrate is in the anionic form. In mitochondria the electric potential of the outer compartment is positive with respect to the interior space so that the picrate ions are prevented from entering the inner space by the electric field. In the inverted submitochondrial particles the membrane potential difference is reversed and picrate anions are driven into the submitochondrial particles. As a result the submitochondrial particles, but not mitochondria, are sensitive to picrate.

These two results of biophysical studies are intended to illustrate that understanding of the action of biologically active compounds in artificial lipid membranes is useful for the interpretation of their biological effects.

It is necessary that physical models of the action of uncouplers in membranes be able to reconcile (a) the $\mathrm{pH}$ dependence of uncoupler induced membrane conductivity with the $\mathrm{pH}$ dependence of uncoupling activity, and (b) the concentration dependence of membrane conductivity with the concentration dependence of uncoupling activity. Again, within the concept of the chemiosmotic hypothesis it is to be expected that the above dependencies be identical. This project was motivated by the 
above concerns. We have studied the effect of pentachlorobenzenethiol on the conductivity of lipid membranes because the results of other investigators were in conflict with the chemiosmotic concept $(34)$. Wilson et. al. (34) measured the pH dependence of uncoupling activity of pentachlorobenzenethiol in rat liver mitochondria, and the $\mathrm{pH}$ dependence of pentachlorobenzenethiol induced conductivity in lipid bilaytr membranes. These authors reported on absence of correlation between the $\mathrm{pH}$ dependence of membrane conductance and $\mathrm{pH}$ dependence of uncoupling activity. In the present project we have performed careful experiments and have found experimental conditions which made it possible to study the effect of pentachlorobenzenethiol induced conductivity in greater detail. We have found that our data on pH dependence of induced conductance in lipid bilayers and the Wilson et al. (34) data on uncoupling activity in mitochondria are very similar (Fig. 42), as is predicted from chemiosmotic concept. Another relationship that follows from the chemiosmotic theory is that the dependence of membrane conductance on concentration of uncoupler and the dependence of uncoupling activity on uncoupler concentration are to be identical. This problem has not yet received sufficient attention. It was noted that for the uncoupler DTFB that the dependence of uncoupling activity on DTFB concentration was linear whereas the concentration dependence of DTFB induced conductance was quadratic (9). It was also found that electrical conductance of lipid membranes prepared with chlorodecane as solvent was linearly, instead of quadratically, dependent on DTFB concentration (9). The increase of membrane dielectric constant, due to the presence of chlorodecane results in the decrease of the potential energy of DTFB anions in the 
membrane; these then become the dominating permeable ion and brings about the transition from quadratic to linear concentration dependence. It has been proposed $(9,35)$ that similar effects occur in biomembranes because of the existence of membrane bound proteins which increase the dielectric constant of biomembranes compared to that of lipid bilayer membranes. It has been shown that the concept of higher dielectric constant can, in principle, reconcile both the uncoupling and conductivity effects.

POTENTIAL ENERGY BARRIERS TO ION TRANSPORT IN MEMBRANES

It is well known that membrane interior presents a potential energy barrier for penetrating ions. The major contribution to the height of the barrier comes from the Born charging energy

$$
w_{0}=\frac{z^{2} e^{2}}{2 k \operatorname{Tr}}\left(\frac{1}{\varepsilon_{m}}-\frac{1}{\varepsilon_{\omega}}\right),
$$

which represents the energy required to move an ion of radius $r$ and charge ze from the aqueous phase of dielectric constant $\varepsilon_{\omega}$ into the memtiane approximated by a thick slab of dielectric constant $\varepsilon_{m} ; k$ is the Boltzmann constant and $\mathrm{T}$ is the absolute temperature. However, this expression is only an approximate one because the membrane, being only about 50-100 \& thick, cannot be treated as a truly macroscopic phase. An ion entering the membrane $\left(\varepsilon_{\mathrm{m}}=2\right)$ from the aqueous phase $\left(\varepsilon_{\omega}=80\right)$ is subject to an image force due to the polarization of the membrane-water interface. As a result an ion in the aqueous phase near the membrane will experience a force away from the membrane, and the ion in the membrane near the interface will experience a force towards the interface. Because membrane has two interfaces at a distance comparable to 
the range of image forces, one has to consider an infinite number of image planes on either side of the membrane in the electrostatic treatment of the problem. This problem has been solved using various approximations (38-40). The shape of the image charge barrier for different values of the membrane phase dielectric constant is shown in Fig. 7. The dashed lines indicate the Born charging energies, corres-

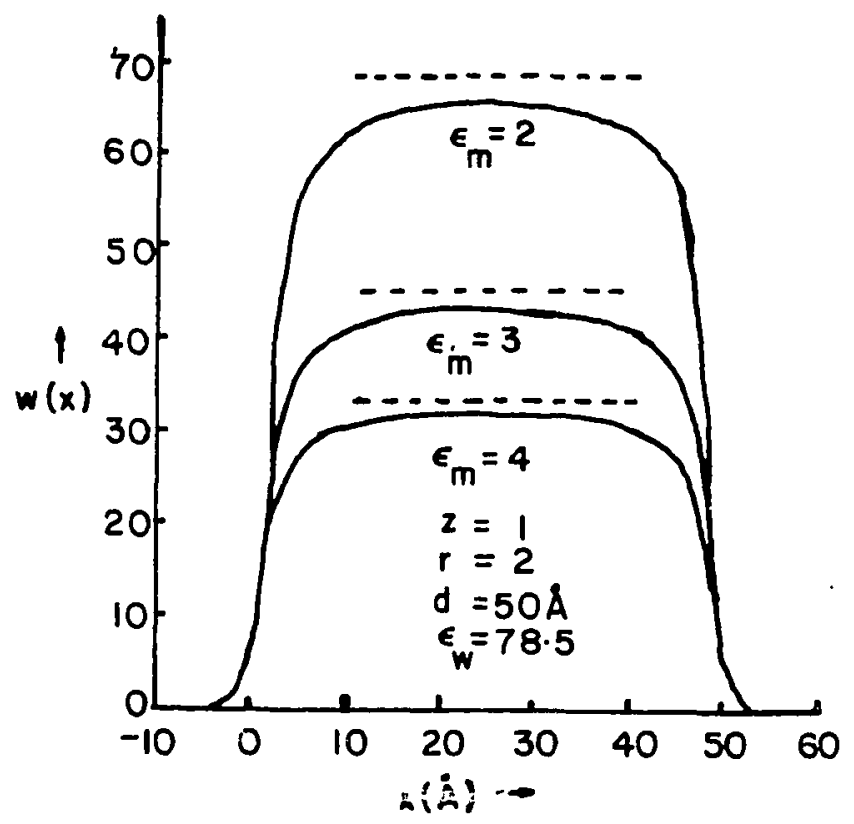

Figure 7. The image charge potential energy barrier experienced by an ion in a bilayer membrane. $\mathbf{z}=$ valency of the icn; $r=$ radius of the ion, $d=$ thickness of the membrane; $\varepsilon_{m}, \varepsilon_{\omega}=$ dielectric constants of the membrane and water respectively. $W(x)$ is in units of kT. The dashed lines are the Born charging energies calculated using Eq. 2 for the same parameters. This figure is reproduced from reference 40 . 
ponding to infinitely thick medium, for the same parameters. At the center of the barrier the Born charging energies do not differ significantly from those obtained by image charge calculations. An expression for the image charge barrier potential energy $w(x)$ is derived in reference $(38)$.

In case of certain large organic ions, such as tetraphenylborate or the dipicrylamine anions, ion entrance into the membrane is favored by rather strong hydrophobic forces (41). Due to chemical interactions between the entering particle and the membrane there is a decrease of free energy; with regard to the hydrophobic interaction the membrane acts as a potential energy well. The resulting membrane potential energy profile for the entering ion is the sum of the image charge barrier and the hydrophobic energy well (Fig. 8). It shows two potential wells at the membrane water interface which are associated with the ion adsorption planes.

JUSTIFICATION OF DESCRIPTIOIN OF MEMBRANE ION TRANSPORT BY EQUATIONS OF CHEMICAL KINETICS

Electrodiffusion equations, which are strictly applicable only to macroscopic media, can be used to describe the motion of an ion in a membrane, provided that the potential energy barrier $W(x)$ is also taken into account. The flux $j$ is given by

$$
j(t)=-c u d \mu / d x,
$$

where $c=$ ion concentration in the membrane,

$$
u=\text { mobility, }
$$

and $\mu=$ electrochemical potential energy of the ion in the membrane. The electrochemical potential energy is given by 


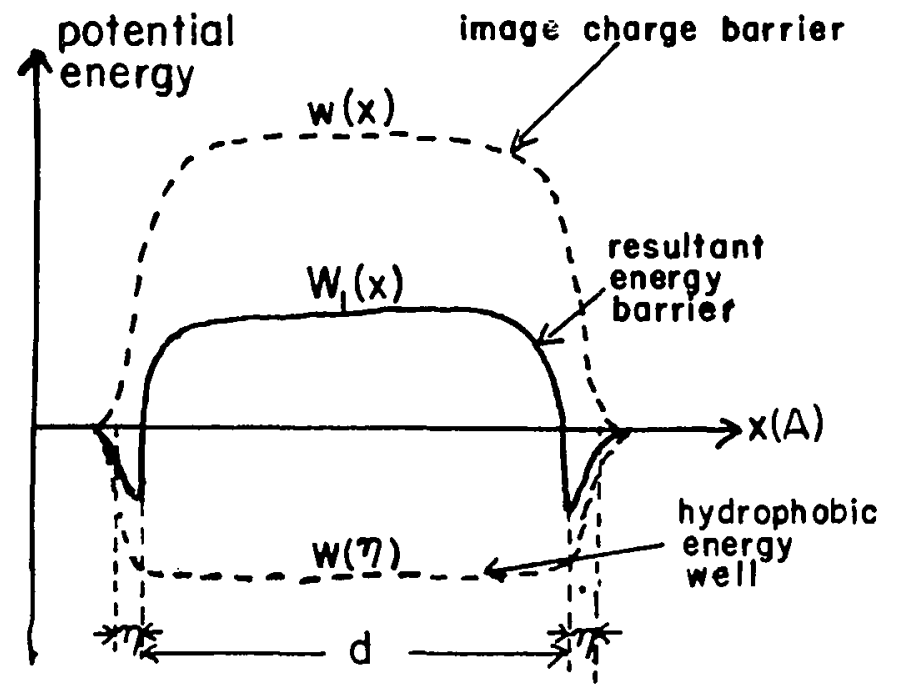

Figure 8. Component and the resultant potential energy barrier for hydrophobic ions in a bilayer membrane.

$$
\mu=\mu_{0}+k T \ln (c)+z e \Psi,
$$

where $\mu_{0}$ is a constant and $z$ is the valency of the ion. ze $\psi(x)$ represents the potential energy of the ion in the membrane and consists of two terms. One is the electric potential energy $z e \phi(x)$ due to the externally applied electric field, and the other term, $w(x)$, is the potential energy of the interaction of the ion with the polarization charges at the membrane water interface (Fig. 7). Therefore,

$$
j(t)=-c u\left(\frac{k T}{c} \frac{d c}{d x}+z e \frac{d \phi}{d x}+\frac{d W}{d x}\right)
$$

Using Einstein's relation $u=D / k T$ where $D$ is the diffusion coefficient, 


$$
j(t)=-D \frac{d c}{d x}-\frac{c D}{k T} z e \frac{d \phi}{d x}-\frac{c D}{k T} \frac{d W}{d x}
$$

The concentration $c$ is a function of the time and position. A relationship between the flux $j$ across the membrane and the concentration of ions at the membrane water interface is obtained by integrating Eq. 6 between the left ion adsorption plane at $x=\eta$ and the right ion adsorption plane at $x=d-n$. Denoting $c$ at $x=n$ by $c^{\prime}$ and $c$ at $x=d-n$ by c" we obtain

$$
j(t)=\frac{c^{\prime} \exp (-z e \beta V / 2 k T)-c^{\prime \prime} \exp (z e \beta V / 2 k T)}{\exp (-z e V / 2 k T)_{\eta^{J}} d-D^{-1} \exp \left[w_{1}(x)+z e \Phi(x)\right] d x},
$$

where $w_{1}(x)=W(x)-W(n)$ is the ion potential energy relative to that at the adsorption plane (Fig. 8). Coefficient BV is the fraction of the applied voltage effective in driving transmembrane transport $\{B=$ $(d-2 n) / d\}$. Anderson and Fuchs (38) have shown that Eq. 7 can be written in a more convenient form

$$
j(t)=\frac{c^{\prime} \exp (-z e B V / 2 k T)-c^{\prime \prime} \exp (z e B V / 2 k T)}{\exp \left[\omega(z e V / k T)^{2}\right]_{\eta^{\prime}} d-\eta_{D}{ }^{-1} \exp \left[w_{1}(x) / k T\right] d x}
$$

Parameter $w$ is dependent on the membrane thickness and has been tabulated for various membrane thicknesses in reference (38). The above electrodiffusion model of ion transport has been experimentally verified and found satisfactory (38).

The form of Eq. 8 can be used to illustrate the equivalence between the electrodiffusion and kinetic treatment of ion transport in 
membranes. According to the concepts of chemical kinetics, the net flux of ions across the membrane is given by the difference of the partial fluxes

$$
j(t)=-d N^{\prime} / d t=k_{1} N^{\prime}-k_{2} N^{\prime \prime}=d N^{\prime \prime} / d t
$$

where $k_{1}$ represents the rate constant for ion translocation from left to right and $k_{2}$ represents the rate constant for ion translocation from right to left. $N^{\prime}$ and $N^{\prime \prime}$ are the ion densities per unit area at the left and right adsorption planes. From the comparison of Eq. 8 with Eq. 9 it is obvious that the electrodiffusion and kinetic treatment of membrane transport are equivalent. The relationships between $N^{\prime}, N^{\prime \prime}$ and $c^{\prime}, c^{\prime \prime}$ are $N^{\prime}=c^{\prime} \gamma_{\omega}$ and $N^{\prime \prime}=c^{\prime \prime} \gamma_{\omega}$ where $\gamma_{\omega}$ is the width of the ion adsorption well at the interface. The rate constants $\mathrm{k}_{1}$ and $\mathrm{k}_{2}$ are voltage dependent due to the change of the height of the membrane potential energy barrier by the applied voltage. The explicit forms for the ion translocation rate constants are

$$
\begin{aligned}
& k_{1}=k_{0} \exp \left(-w(z e V / k T)^{2}\right) \exp (-z e \beta V / 2 k T), \\
& k_{2}=k_{0} \exp \left(-w(z e V / k T)^{2}\right) \exp (z e \beta V / 2 k T) \\
& k_{0}=\left[n^{d-n} D^{-1} \exp \left(w_{1}(x) / k T\right) d x\right]^{-1}
\end{aligned}
$$

is the rate constant for translocation of ions in the absence of an electric field. Eq. 12 shows the relationship between the ion translocation rate constant and the diffusion coefficient in the membrane interior and the relative height of membrane potential energy barrier. Since $w$ is of the order of $10^{-3}$ (38) the variation of the factor $\exp \left(-\omega(z e V / k T)^{2}\right)$ with voltage is small. Hence in the analysis of 
kinetic models we neglect this term. Since there is no indication that the ions we studied are adsorbed at a considerable distance below the membrane surface, we also take $\beta=1$. Then for negatively charged monovalent ions (ze $=-1$ ) such as ions derived from chlorinated phenols we have

$$
k_{1}=k_{0} \exp (e V / 2 k T)
$$

and

$$
\mathrm{k}_{2}=\mathrm{k}_{0} \exp (-\mathrm{eV} / 2 \mathrm{kT})
$$

\section{MODELS OF MEMBRANE TRANSPORT}

Eqs. 13 and 14 are adequate to describe the transport of highly hydrophobic ions such as tetraphenylborate (see Chapter V) through membranes. At low aqueous concentrations the rate of interfacial transfer of such ions across the membrane water interface is slow $(38,42)$, so that when a potential difference is applied across the membrane one observes only a transient current arising from the redistribution of ions between the two membrane surfaces.

Ionophores like nonactin and valinomycin, on the other hand, form membrane permeable complexes with monovalent cations like $\mathrm{K}^{+}$and therefore the treatment of ionophore mediated transport require multistep kinetics. It is usually the membrane bound ionophore that associates with the cation from the aqueous phase and after diffusing to the other side of the membrane, it releases the cation to the aqueous phase at the opposite membrane surface. The unloaded ionophore molecule, being highly permeable in the membrane because it does not experience the electrostatic potential energy barrier, then diffuses back to the 
original side of the membrane. This mode of membrane transport has been extensively studied and appears to be well understood (43).

Class I uncouplers induce conductivities which depend linearly on the uncoupler concentration. In this case the membrane permeable ion. is most probably the anionic form of the uncoupler $(30,5)$. In steady state the flow of uncoupler anions is supported by an equal and opposite flow of neutral uncoupler molecules. This mechanism has been extensively studied in the classic paper by LeBlanc (5) who also took into consideration the aqueous unstirred layers in membrane vicinity. He also developed a method to determine the membrane permeability of the neutral form of class I uncouplers. The method is based on the measurements of the membrane potential produced in response to hydrogen ion concentration gradients across the membrane. If the transport limiting effects caused by aqueous unstirred layers are insignificant, then the ion transport induced by class I uncouplers can be described by a model very similar to that of carrier mediated transport induced by ionophores (see Chapter V).

The membrane permeable ion in the case of class II uncouplers was assumed to be a dimer $\mathrm{HA}_{2}^{-}$formed by the association of a neutral HA and an anionic $A^{-}$form of the uncoupler $(44,45)$. This assumption explains both the concentration dependence and the $\mathrm{pH}$ dependence of conductivity induced by class II uncouplers and is supported by the discovery of existence of a similar complex by Poonia (46). The complex $\mathrm{HA}_{2}^{-}$can be formed either in the aqueous phase or at the membrane. If this dimer was formed in the aqueous solution, then one would expect to observe unstirred layer effects (47), such as membrane current decay on time scale of seconds. These are not experimentally observed. Also the 
maximum conductance expected for charge transport by diffusion through the aqueous unstirred layers is significantly smaller than that observed experimentally $(8,28,30)$. These observations rule out the possibility of the formation of dimers in the aqueous phase. Neumcke et al. (30) considered a number of different bimolecular models of membrane transport consistent with the above mentioned results. They concluded, resting their conclusions on results of current transient experiments performed with the uncoupler TTFB, that the model which best explains the experimental results is one in which the dimers are formed at the membrane surface and in which the flux of dimers through the membrane is supported by an opposite flux of neutral uncoupler molecules. This model (dimer model A) is discussed in detail in Chapter III.

\title{
KINETIC LIMITATIONS
}

\begin{abstract}
One of the important factors illuminating the kinetics of membrane transport are kinetic limitations. Kinetic limitations occur whenever the chemical processes coupled to the transmembrane flow of ions are not fast enough to keep up with or support the otherwise predicted transmembrane flow of ions. In the absence of kinetic limitations the current through the membrane is determined by the membrane potential energy barrier for membrane permeable ions, and by the density of membrane permeabile ions at the membrane water interface. Under these conditions, the initial current, which reflects the initial equilibrium conditions and is observed when a voltage step is first applied across the membrane, does not change with time. It means that no current decay, called relaxation, is observable in the absence of kinetic limitations.
\end{abstract}


The steady state and transient characteristics of membrane transport depend on the type of kinetic limitations that are associated with the different processes of the transport cycle. It is sometimes possible to predict the type of kinetic limitations responsible for the observed experimental results.

It follows from the condition of equilibrium that in the absence of kinetic limitations, and assuming that the transmembrane transport is driven by the full applied voltage, that the normalized membrane conductivity is equal to

$$
G(V) / G(0)=\sinh (e V / 2 k T) /(e V / 2 k T)
$$

where $G(V)$ is the conductivity at the applied voltage $V$, and $G(0)$ is the value of $\mathrm{G}(\mathrm{V})$ when extrapolated to zero voltage. Therefore, if the experimentally obtained normalized membrane conductivity values are lower than those predicted by Eq. 15, it means that kinetic limitations are present. Changes of the normalized membrane conductance with the $\mathrm{pH}$ of aqueous environment and the concentration of uncoupler can be used to gain insight into the type of kinetic Iimitations involved.

We outline the theoretical methods adopted for the analysis of membrane transport models.

OUTLINE OF THEORETICAL METHODS OF ANALYSIS OF MEMBRANE KINETICS

(1) Linear Models of Membrane Transport. Let the membrane transport scheme consist of $s$ components, and let each component interact with every other component. As an illustration consider the scheme shown in Fig. 9. $k_{X Y}$ denotes the rate constant for the conversion of 
component $Y$ to $X$. Let the transmembrane ion translocation be denoted by the reaction $A-B$. Then the ion flux through the membrane is given by

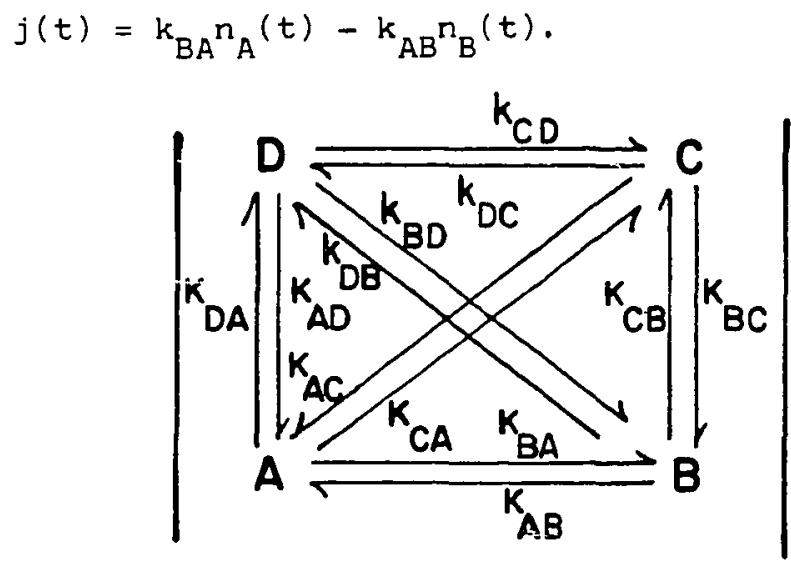

Figure 9. General linear model of membrane transport.

The time dependent densities $n_{A}, n_{B}$ of the membrane permeable ion on either side of the membrane are obtained by solving the set of differential equations 18 given below. The rate of change of the concentration of $A$ is given by

$$
\begin{aligned}
d n_{A} / d t & =-\left(k_{B A}+k_{C A}+k_{D A}+\ldots \ldots \cdot n_{A}+k_{A B} n_{B}+k_{A C} n_{C}+\ldots \cdots\right. \\
& =\Sigma^{s} k_{A \alpha^{n} \alpha} \\
& \alpha=A
\end{aligned}
$$

where $k_{A A}=-\left(k_{B A}+k_{C A}+k_{D A}+\ldots \ldots \ldots\right)$.

Similarly

$d n_{B} / d t=\sum_{\alpha} k_{B} \alpha^{n} \alpha$,

...................

$d n_{S} / d t=\sum_{\alpha} k_{S} \alpha^{n} \alpha$. 
(a) General method of solution. General solution of a set of coupled, linear, homogeneous differential equations is given by

$$
n_{\alpha}=\sum_{j=1}^{s} a_{\alpha j} \exp \left(-\lambda_{j} t\right)+\bar{z}_{\alpha}
$$

where $\bar{n}_{i}$ is the steady state concentration of the component $i$ which is obtained by solving the system for steady state. The constants $a_{\alpha j}$ and $\lambda_{j}$ can be obtained by substituting Eq. 19 into the set of differential Eqs. 17-18.

For component $A$ we get

$\sum_{j=A}^{s} a_{A, j} \lambda_{j} \exp \left(-\lambda_{j} t\right)=\sum_{\alpha} k_{A \alpha} \sum_{j=A}^{s} a_{\alpha j} \exp \left(-\lambda_{j} t\right)+\sum_{\alpha} k_{A \alpha} \bar{n}_{\alpha}$.

Rearranging terms and changing summations

$$
j \sum_{A}^{S} \exp \left(-\lambda_{j} t\right)\left[\sum_{\alpha} k_{A \alpha} a_{\alpha j}+\lambda_{j} a_{A j}\right]+\sum_{\alpha} k_{A \alpha} \bar{n}_{\alpha}=0 .
$$

Eq. 21 is satisfied at all times only if

$$
\sum_{\alpha} k_{A_{\alpha}{ }_{\alpha j}}+\lambda_{j} a_{A j}=0
$$

and

$$
\sum_{\alpha} \mathrm{k}_{A \alpha} \overline{\mathrm{n}}_{\alpha}=0
$$

Similarly for all components we get

$$
\sum_{\alpha} k_{B \alpha} a_{\alpha j}+\lambda_{j} a_{B j}=0
$$

and

$$
\sum_{\alpha} k_{B \alpha} \bar{n}_{\alpha}=0
$$

$$
B=A, B, C, \ldots \ldots, s \text {. }
$$


The system of Eqs. 24 could be written in matrix form as

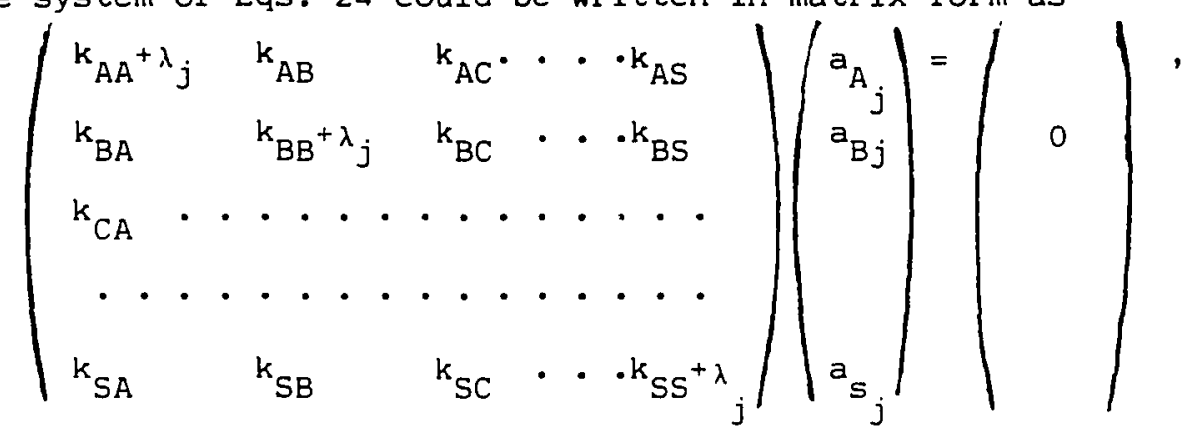

which has a non-trivial solution only if

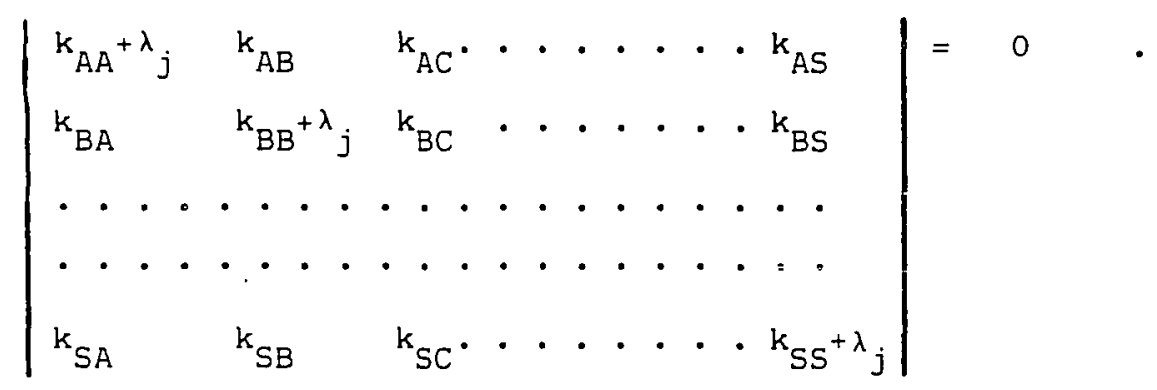

The above secular equation is of degree $s$ in $\lambda_{j}$ and by solving it we obtain s relaxation time constants $\tau_{i}$ given by $\tau_{i}=1 /{ }_{i}$. These relaxation times have a physical meaning only when $\tau_{i}$ is real and positive. Real negative $\tau_{i}$ would mean that the concentrations would increase indefinitely with time and imaginary $\tau_{i}$ would mean that the concentration would oscillate with time. The constants $a_{\alpha j}$ are obtained from the set of Eqs. 26 and the initial conditions. The flux of membrane permeable species through the membrane could be expressed in the form

$$
j(t)=\sum_{j} \alpha_{j} e^{-t / \tau} j+j(\infty)
$$

(b) Solution in terms of Normal Coordinates. The general method of solution discussed in (a) is convenient for obtaining analytical solutions to simpler problems. But for complex systems the formulation 
in the form of an eigenvalue problem is more useful because of the availability of number of computer programs in scientific subroutine libraries which can be efficiently applied to studies of membrane kinetics.

The solution of the membrane transport problem, for the time evolution of transmembrane ion flux, is mathematically similar to the problem of determining eigenfrequencies and eigenvectors of vibrating systems. Instead of a generalized displacement vector in the mechanical problem, it is possible to construct a concentration vector representing the displacements of concentrations or surface densities from the initial equilibrium or from the final steady states. Corresponding to eigenfrequencies the method gives the reciprocals of relaxation time constants.

The problem of membrane transport was set up in the previous subsection (a); according to Eqs. 17 and 18, the rate of change of density of species $B$ is,

$$
d n_{B} / d t=\sum_{\alpha} k_{B \alpha}{ }^{n},
$$

where $B=A, B, \ldots . .$.

and $\quad \alpha=A, B, \ldots S$.

Substituting $\mathrm{X}_{\alpha}$, the displacement of the concentration of the component $\alpha$ from either the initial equilibrium or the final steady state $\bar{n}_{\alpha}$, defined by the Eq. $n_{\alpha}(t)=x_{\alpha}(t)+\bar{n}_{\alpha}$

we obtain a set of first order differential equations for the concentration displacements,

$$
\begin{aligned}
& \mathrm{dx}_{\mathrm{A}} / \mathrm{dt}=\sum_{\alpha} \mathrm{k}_{A_{\alpha}} \mathrm{x}_{\alpha} \\
& \ldots \ldots \ldots \ldots \ldots \\
& \mathrm{dx}_{\mathrm{S}} / \mathrm{dt}=\sum_{\alpha} \mathrm{k}_{S_{\alpha}} \mathrm{x}_{\alpha}
\end{aligned}
$$


since $\sum_{\alpha} k_{\beta \alpha} \bar{n}_{\alpha}=0$ for all components $B$.

The form of Eq. 17 indicates that $\sum_{\alpha} \mathrm{dn} \mathrm{n}_{\alpha} / \mathrm{dt}=0$, which means that $\sum_{\alpha} \mathrm{n}_{\alpha}$ = constant. The same is true for the displacements of concentrations, but in this case the constant is zero, which follows from the definition of $X(\mathrm{Eq} \cdot 30)$. Therefore,

$$
\sum_{\alpha} X_{\alpha}=0
$$

The above result means that these $s$ component concentrations are linearly dependent and thus only (s-1) components are required to completely specify the state of the system. In other words, the number of degrees of freedom of the system is (s-1). We could use the nass conservation Eq. 32 to reduce the system of differential equations from $s$ to (s-1) which would yield (s-1) relaxation time constants.

In matrix notation the set 31 could be written in the form

$$
\{d X / d t\}=\{K\} \quad\{X\}
$$

where $\{d x / d t\}$ and $\{x\}$ are column vectors and $\{K\}$ is the kinetic matrix, whose components are $\mathrm{k}_{B \alpha^{\circ}}$. Let the concentration displacement vector $\{\mathrm{X}\}$ be expressed as a linear combination of another vector $\{Y\}$ by the transformation

$$
\{X\}=\{M\}\{Y\}
$$

Substituting for $X$ in terms of $Y$ in Eq. 33 we arrive at

$$
\{d Y / d t\}=\left\{M^{-1}\right\} \quad\{K\} \quad\{M\} \quad\{Y\}
$$

If the transformation matrix $\{M\}$ is selected in such a way that after the similarity transformation $\left\{M^{-1}\right\}\{K\}\{M\}$ the new matrix is diagonal 


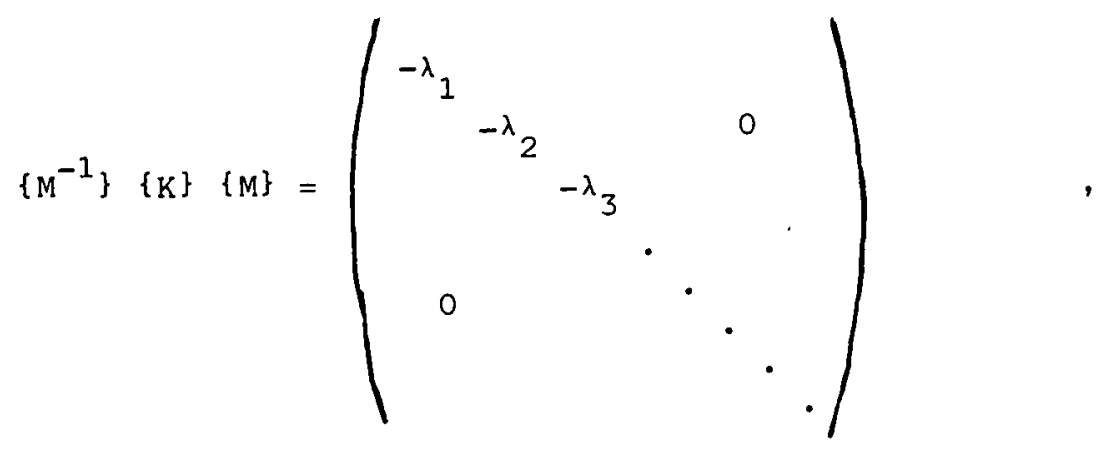

the system of differential equations then becomes decoupled;

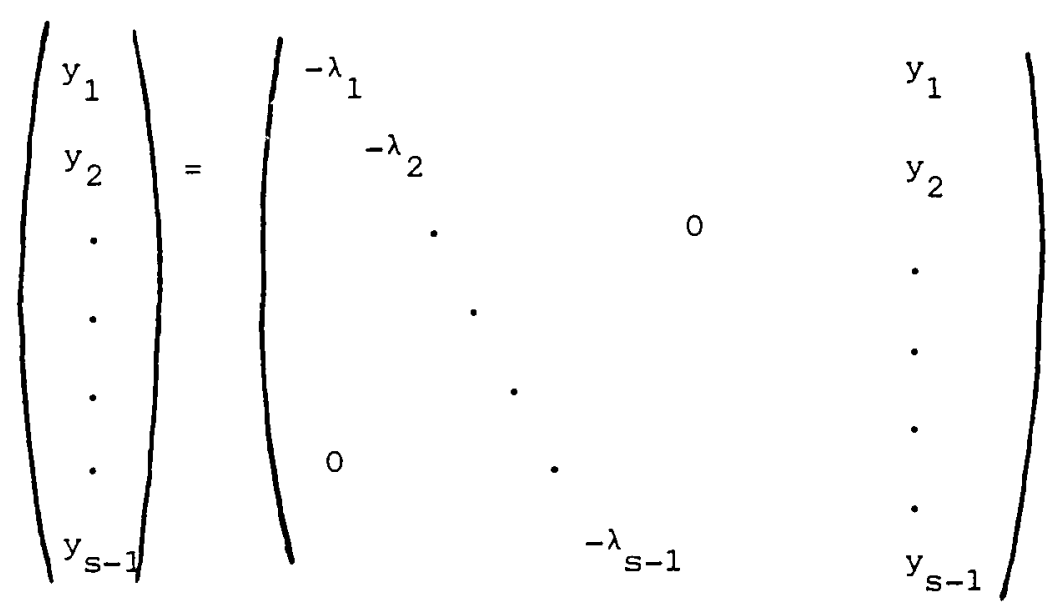

and it has solutions

$$
\begin{aligned}
& Y_{i}=Y_{i 0} \exp \left(-\lambda_{i} t\right)=Y_{i 0} \exp \left(-t / \tau_{i}\right) \\
& i=1,2,3, \ldots,(s-1) .
\end{aligned}
$$

$Y_{i}(i=1,2,3, \ldots, s)$ are the normal coordinates and $y_{i 0}$ are the initial amplitudes of the normal coordinates.

Since the eigenvalues do not change under a similarity transformation, it follows that the diagonal elements of the matrix $\left\{M^{-1}\right\}\{K\}\{M\}$ are equal to the eigenvalues of the kinetic matrix $\{K\}$ obtained from the eigenvalue Eq. 
$\{K\}\{a\}=-\lambda_{j}\{a\}$,

which is the same as Eq. 26.

Eq. 38 could be transformed back to the original coordinates $x$ by operating on it by the transformation matrix $\{M\}$. This operation yields

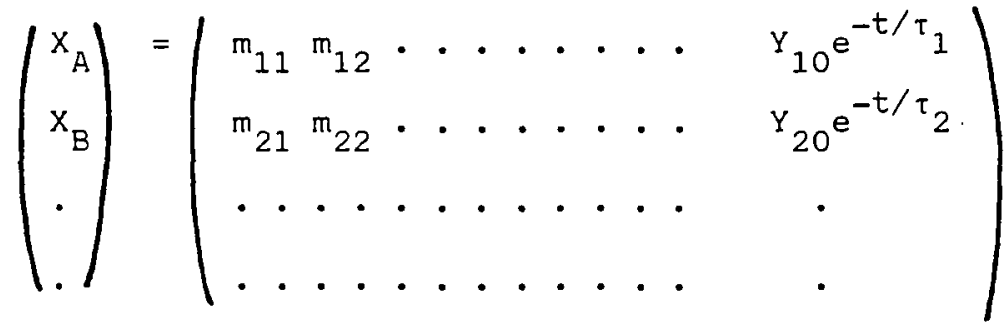

The displacement amplitudes $Y_{i 0}$ of the normal coordinates are obtained from the initial conditions. The transmembrane flux is given by

$$
\begin{aligned}
j(t) & =k_{B A} \cdot n_{A}-k_{A B} \cdot n_{B} \\
& =k_{B A}\left(x_{A}+\bar{n}_{A}\right)-k_{A B}\left(x_{B}+\bar{n}_{B}\right) \\
& =k_{B A} \cdot x_{A}-k_{A B} x_{B}+j, \quad \text { where } \bar{j}=k_{B A} \cdot \bar{n}_{A}-k_{A B} \cdot \bar{n}_{B} \\
& =\sum_{i} j_{i O} \cdot \exp \left(-t / \tau_{i}\right)+\bar{j}
\end{aligned}
$$

The initial flux is

$$
j_{i 0}=Y_{i 0}\left(k_{B A} \cdot m_{1 i}-k_{A B} \cdot m_{2 i}\right) \quad i=1,2,3, \ldots
$$

(2) Nonlinear Models of Membrane Transport. A nonlinear membrane transport model consists of schemes of the following general type

$r_{p A} n_{A}+r_{p B} n_{B}+r_{p C} n_{C}+\cdots \cdots \cdot \frac{k_{p f}}{k_{p r}} r_{p T_{T}}+r_{p U} n_{U}+r_{p V} n_{V}+\cdots \cdots$

where $p$ denotes one of the reaction pathways. The $r^{\prime} s$ are the stoichiometric coefficients and the n's are the surface densities of individual components. From the theory of chemical kinetics it follows that the rate of the forward and the reverse processes for this particular 
reaction are given by

$-\left.\frac{d n_{A}}{d t}\right|_{p f}=k_{p r_{A}^{n}}^{n_{p A}} \cdot n_{B}^{r_{p B}} \cdot n_{\tilde{C}}^{r_{p C}}$

and $\left.\quad \frac{d n^{A}}{d t}\right|_{p r}=k_{p r}{ }^{n_{T}}{ }^{p T} \cdot n_{U}^{r} p U \cdot n_{V}^{r p V}$

The rate of change of any component $\alpha$ is obtained by summing over all reaction pathways in which the component a take part.

$\frac{d n_{\alpha}}{d t}=\sum_{p}\left[-k_{p f_{A}^{n_{A}}}^{r_{p A}} \cdot n_{B}^{r_{p B}} \ldots \ldots+k_{p r} n_{T}^{n_{p T}} \cdot n_{U}^{{ }^{r} p U} \ldots \ldots\right.$.

No general analytical method of solution exists for the exact solution of this system. Therefore either numerical integration methods or approximate analytical solutions by linearization around the initial equalibrium or final steady state must be adopted.

The linearization process proceeds as follows. Let $\bar{n}_{\alpha}$ represent either the initial equalibrium state or the final steady state surface density of the component $\alpha$. Then we can define the displacement density $x_{\alpha}$ by $n_{\alpha}=x_{\alpha}+\bar{n}_{\alpha}$. The rate of change of density displacement in the vicinity of the reference state is then given by

$$
\begin{aligned}
& \overline{d x}^{\alpha}=\sum_{p}-k_{p f}\left[\left(r_{p A} \bar{n}_{A}^{\left(r_{p A}^{-1}\right)} x_{A}\right) \bar{n}_{B}^{r} p B \cdot \bar{n}_{C}^{r} p C \ldots \ldots+\bar{n}_{A}^{r_{p A}}\left(r_{p B} \bar{n}^{\left(r_{p B}-1\right)} x_{B}\right) \bar{n}_{C}^{r} p C \ldots\right] \\
& \left.+\sum_{p} k_{p r}\left[\left(r_{p T} \bar{n}_{T}^{\left(r_{p T}-1\right)} x_{T}\right) \bar{n}_{U}^{r} p U \cdot \bar{n}_{V}^{r} p V \ldots+\bar{n}_{T}^{r} p T\left(r_{p U} \bar{n}_{U}^{\left(r_{p U}-1\right)} x_{U}\right) \bar{n}_{V}^{r} p V\right) \ldots\right]
\end{aligned}
$$


The above result was obtained by using the binomial expansion and retaining only the linear terms in $x$. This implies that $x_{\alpha} \ll \bar{n}_{\alpha}$ i.e. the displacement of the system from the reference state is very small. Eq. 47 can be written in a simpler form;

$$
\frac{d X_{\alpha}}{d t}=\sum_{B}\left[\frac{\partial}{\partial x_{B}}\left(\frac{d n_{B}}{d t}\right)\right] x_{\alpha}
$$

This is a system of linear, first order differential equations and can be exactly solved for by the methods outlined in the preceding subsection. The linearization method is a useful technique for systems which can not be displaced significantly from the reference state. It is expected that, depending on the reference state, the linearized transport models will provide an adequate description of the transient membrane flux in two cases; (a) in the initial phase of the relaxation process i.e. in the short time domain, when the fast processes dominate the relaxation amplitude, or (b) in the final phase, i.e. in the long time domain, when the relaxation is dominated by the slowest process. 


\title{
CHAPTER II
}

\section{METHODS AND MATERIALS}

MATERIALS

\begin{abstract}
Membranes and lipid vesicles were prepared from egg lecithin (phosphatidylcholine) (Fig. 10) and mixtures of egg lecithin and cholesterol, respectively. Egg lecithin was prepared by Dr. Kwan Hsu by the method described in (28). Recrystallized cholesterol was a gift from Dr. David McClure of the Chemistry Department. Pentachlorophenol (PCP) (Fig. 11.a) and n-decane were purchased from Aldrich Chemical Company. Pentachlorobenzenethiol (PCBT) (Fig. 11.b) was purchased from Dupont (RPA No. 6), and purified by Dr. Kwan Hsu according to the methods described in (48). 2,4,5-Trichlorophenol (2,4,5-TCP) (Fig. 11.c) was purchased from Aldrich Chemical Company and was purified by recrystallization.
\end{abstract}

MEMBRANE EXPERIMENTS

Black lipid membranes were formed by the brush technique on a $2.0 \mathrm{~mm}$ diameter hole in a wall of a TFE (tetrafluoroethylene resin) cell. The voltage applied across the membrane was measured with a digital multimeter (DANA Laboratories, Inc., model 4200 ). The steady state current through the membrane was measured with an electrometer (Princeton Applied Research Model 135), or a digital multimeter (Keithley Instruments Inc., Model 160B). Generally, fiber tip calomel elec- 


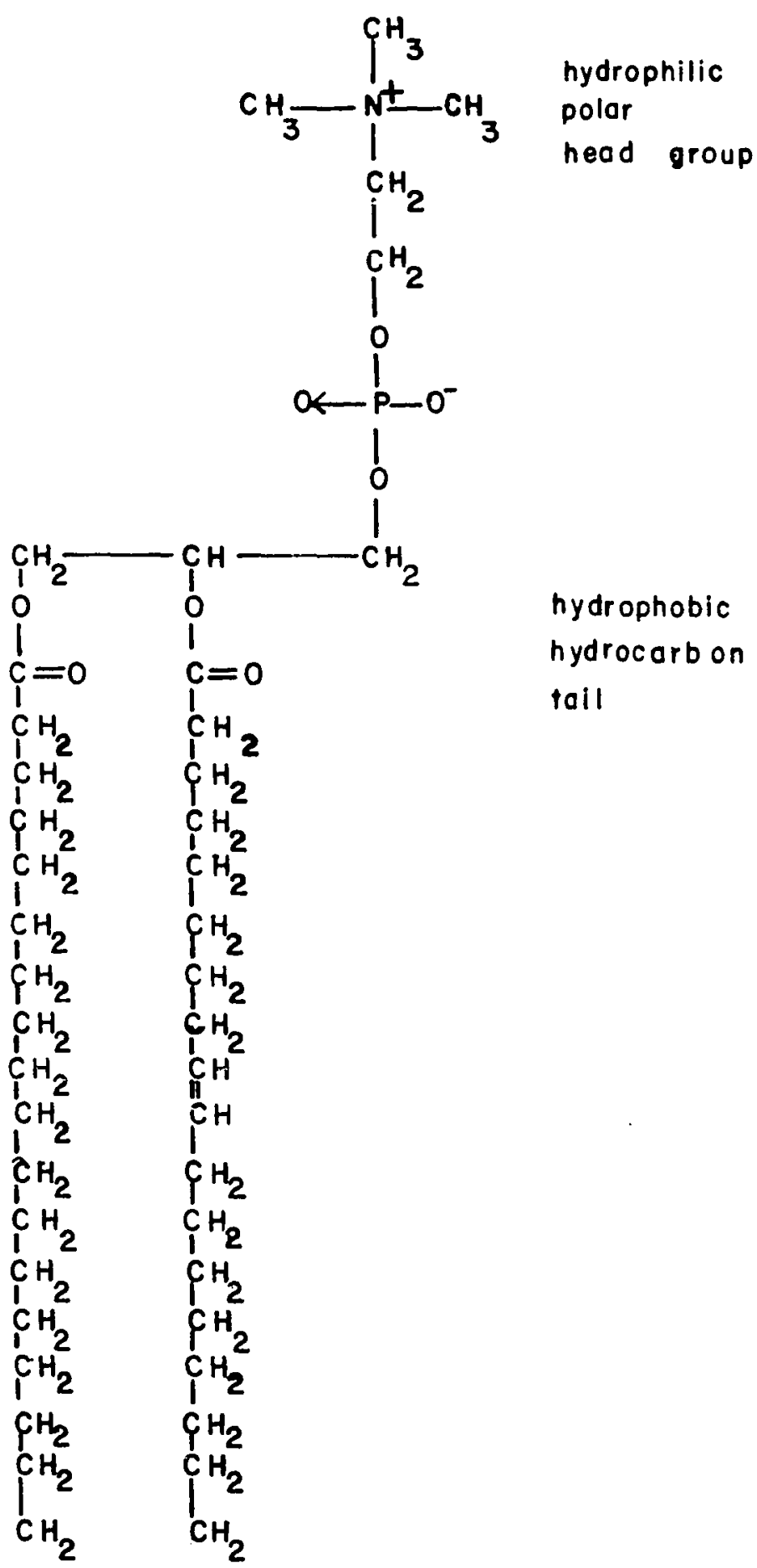

Figure 10. Structure of phosphatidylcholine. 


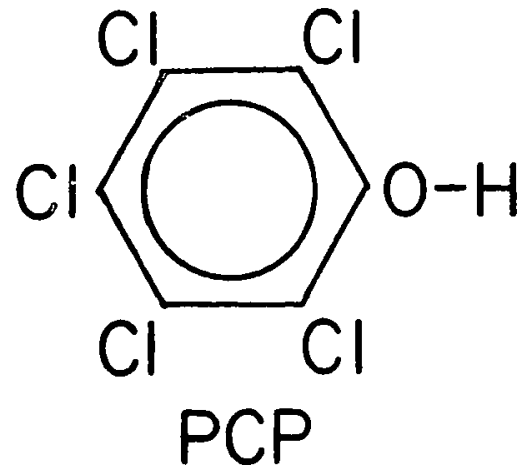

Figure 11.a. Structure of pentachlorophenol.

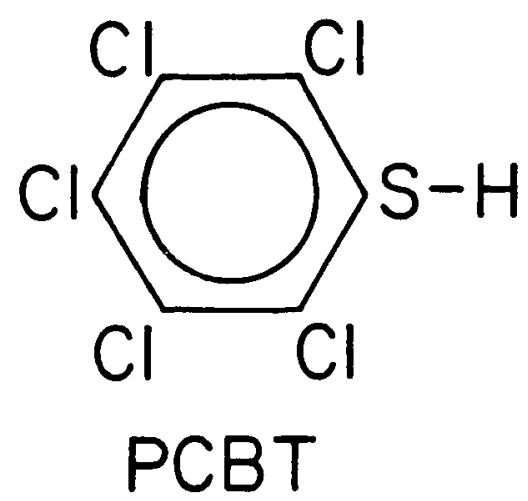

Figure 11.b. Structure of pentachlorobenzenethiol.

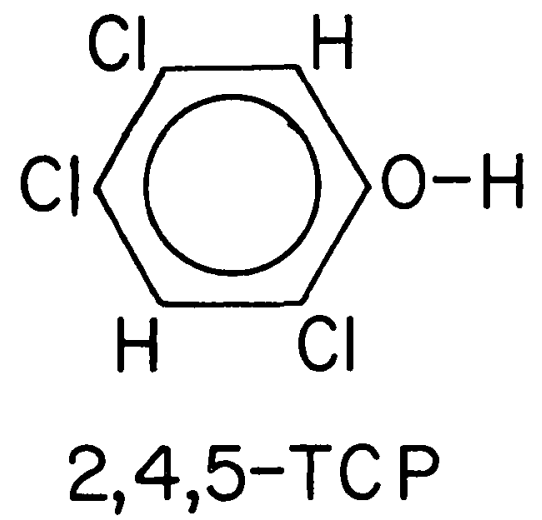

Figure 11.c. Structure of 2,4,5-trichlorophenol. 
trodes (Corning) were used; when the membrane resistance became comparable with the internal resistance of calomel electrodes, Ag/AgCi electrodes (Annex Instruments) were used.

The membrane forming solution contained egg lecithin, cholesterol, and membrane modifier (PCP or PCBT) dissolved in n-decane. The lipid content was approximately $10 \mathrm{mg} / \mathrm{ml}$. All solutions containing PCBT were kept in the dark, sealed under nitrogen and were spectroscopically monitored for PCBT decay. The aqueous medium contained $0.5 \mathrm{M} \mathrm{KC1}$, and phosphate/citrate/borate buffer at concentrations $0.02 / 0.02 / 0.005 \mathrm{M}$, unless otherwise mentioned. At this high buffer concentration the effects due to "unstirred layers" (47) were unimportant. All measurements were done at room temperature $\left(21-23^{\circ} \mathrm{C}\right)$. The steady state current-voltage characteristics were measured after the current stabilized, which was monitored by periodic application of $25.0 \mathrm{mV}$ pulses. Membrane conductance was calculated using the area of the hole.

It was essential to incorporate PCBT into the membrane forming solution, because if PCBT was present only in the aqueous medium, the membrane conductance would increase very slowly and never reach a stationary level. Incorporation of PCBT into the membrane forming solution also avoided any artifacts associated with the presence of PCBT suspensions in the aqueous medium due to low solubility of PCBT in water. In experiments with the uncoupler 2,4,5-TCP the uncoupler was dissolved in the aqueous solution and not in the membrane forming solution in order to increase the stability and the lifetime of the membrane.

The escape of PCBT from the membrane and the membrane torus into the aqueous solution did not represent a serious problem, because the 
efflux is very small due to the very low solubility of PCBT in water. The concentration of PCBT in the membrane is maintained by its diffusion from the membrane torus, which acts as a PCBT reservoir. In the case of PCP the membrane current gradually decreased with time at high pH. Under such circumstances, the data were taken as soon as an apparent equilibrium was reached. The data points represent an average obtained on at least four membranes.

\section{CURRENT TRANSIENT MEASUREMENTS}

The membrane current relaxation experiments were performed using the apparatus originally designed by sargent (49) and schematically shown in Fig. 12. A voltage step was applied across the membrane and the resulting current transient was amplified, digitized and stored in the transient recorder (Biomation, Model 802). The data were displayed on an oscilloscope for inspection and finally recorded on a X-Y plotter (Houston Instruments, Omnigraphic Model 2000). The transient characteristics, the relaxation amplitude $\alpha$ and the time constant $\tau$, were obtained by a least square fit of the current decay at long times (i.e. after the amplifier had fully recovered from the membrane capacitive current overload) to the equation

$$
I_{t}=I_{\infty}[1+\alpha \exp (-t / \tau)]
$$

where

$$
\alpha=\left(I_{0}-I_{\infty}\right) / I_{\infty}
$$

and where

$$
\begin{aligned}
& I_{0}=\text { initial current }(i . e \cdot \text { at } t=0) \\
& \left.I_{\infty}=\text { steady state current (i.e. at } t=\infty\right)
\end{aligned}
$$

and

The amplitude and the time constant of transient currents induced by the uncouplers were for a wide range of experimental conditions at or 


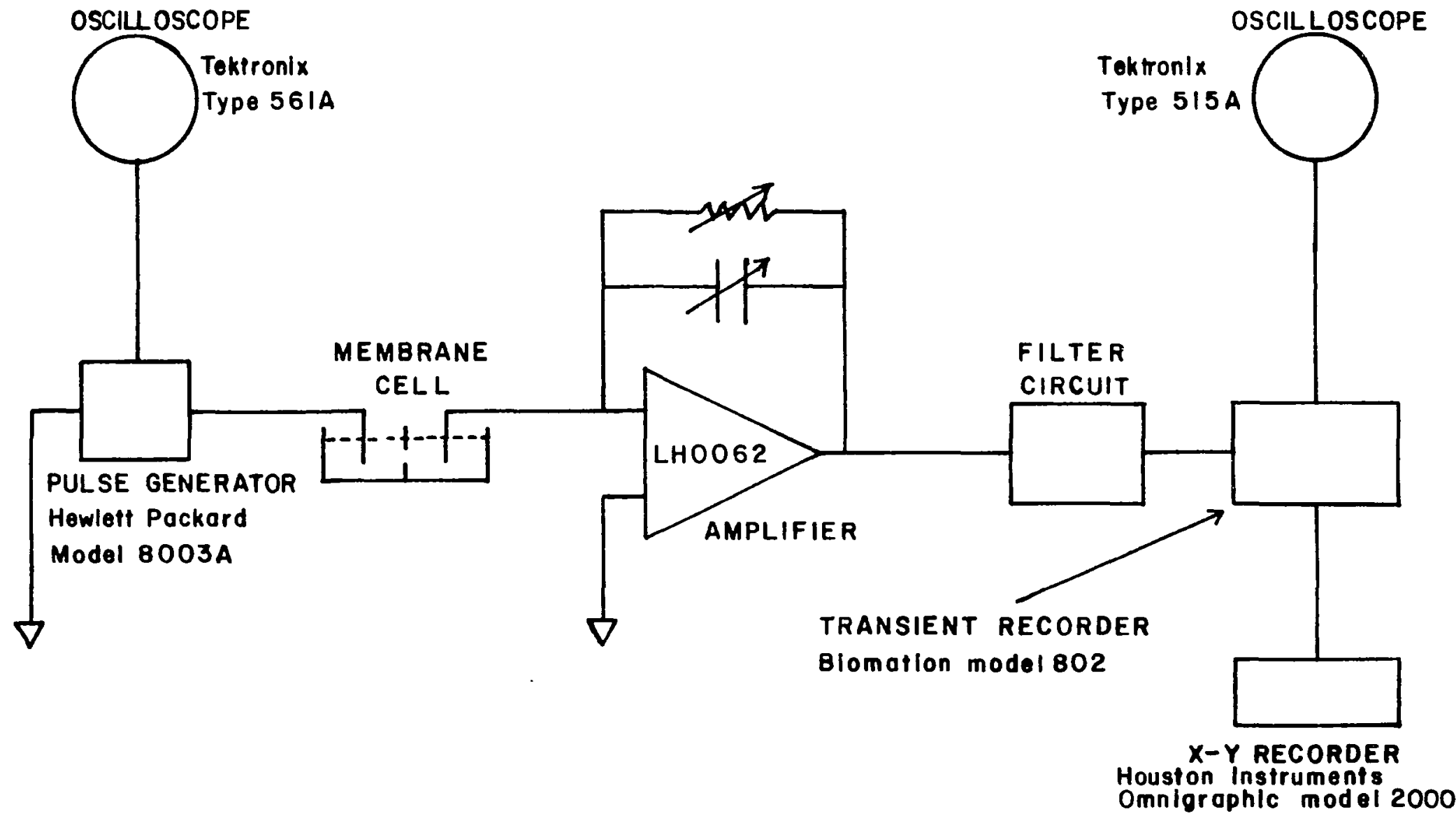

Figure 12. Block diagram of the setup used for current transient experiments. 
below the detection and time resolution limit of the available equipment. It was possible to measure the membrane current decay with time constant greater than 20 microseconds for currents exreeding $10^{-7}$ emps; for currents of the order of $10^{-8}$ amps the time resolution limit was about 50 microseconds. These limits were due to slow amplifier recovery from the membrane capacitive current overload, and the noise. An attempt to increase membrane current by increasing membrane area was unsuccessful. Membranes with larger area were less stable, and, in addition, an increased capacitive component of the amplifier input impedance degraded the performance of the amplifier. The diode clipping circuit used to limit amplifier gain in the time domain when the measured current is dominated by the charging current of the membrane had a rather small beneficial effect. Although the amplifier recovery time was decreased, the diode capacitance also decreased amplifier speed. As a consequence of a poor signal to noise ratio for membrane currents below $5 \times 10^{-9}$ amps it was not possible to obtain reliable data for the relaxation amplitude for PCP and 2,4,5-TCP. For PCBT, which induced greater and slower transient currents than PCP and 2,4,5-TCP, some kinetic studies were possible. However, these studies were limited by the short lifetime of PCBT-containing membranes.

It was concluded that detailed membrane kinetic studies on the above compounds should be done after substantial improvement of the performance of the experimental setup. It is important to have a faster and lower noise amplifier and to use a fast data acquisition system which would make it possible to perform signal averaging and to collect a large amount of data within the timespan of membrane stability. 
MICROELECTROPHORESIS EXPERIMENTS

Multilamellar vesicles (Fig. 5) were prepared from lecithin by drying a solution of lecithin in "gold label" ethanol in a rotary evaporator and then shaking with a solution of buffer and KCl. We found it not necessary to use glass beads as mentioned in reference (50). In the final solution the concentration of $K C 1$ was $0.05 \mathrm{M}$ and the phosphate/citrate/borate buffer was at concentration $0.002 / 0.002 / 0.0005$ M. The velocities of the vesicles were measured on a commercially available (Rank Bros. Bottisham, Cambridge, England) cylindrical cell microelectrophoresis apparatus based on the design of Bangham, Flemans, Heard and Seaman (51). The conductivity $\mathrm{K}$ of the solution was measured using a conductivity meter (Markson, Model 10). The electric field $E_{e}$ in the electrophoretic cell capillary acting on the vesicles was determined according to the formula $I_{e}=K E_{e} c_{c}$, where $I_{e}$ is the current through the cell which was maintained at $500 \pm 10$ microamps, and $A_{C}$ is the area of cross section of the cell capillary. The mobility $\mu$ is given by $\mu=V_{e} / E_{e}$, where $V_{e}$ is the electrophoretic velocity of the vesicles, which is the velocity imparted to the vesicles by the applied electric field.

\section{ALGAL TOXICITY EXPERIMENTS}

Cultures of a green alga, Selenastrum capricornutum, were grown in standard AAP-EPA medium slightly modified as given in Table I. Stock cultures were maintained at $25-27^{\circ} \mathrm{C}$ under continuous fluorescent light $\left(1.09 \times 10^{4} 1 \mathrm{ux}\right)$, and kept in uniform suspension by shaking at 40 cycles/min. The stock cultures were subcultured once in every 3-4 days 
TABLE I

MODIFIED STANDARD AAP-EPA MEDIUM FOR ALGAE

Macronutrients and their final concentrations in $\mathrm{mg} / 1$ :

$\begin{array}{lr}\mathrm{NaNO}_{3} & 25.500 \\ \mathrm{~K}_{2} \mathrm{HPO}_{4} & 1.044 \\ \mathrm{MgCl}_{2} \cdot 6 \mathrm{H}_{2} \mathrm{O} & 12.164 \\ \mathrm{MgSO}_{4} \cdot 7 \mathrm{H}_{2} \mathrm{O} & 14.700 \\ \mathrm{CaCl}_{2} \cdot 2 \mathrm{H}_{2} \mathrm{O} & 4.410 \\ \mathrm{NaHCO}_{3} & 15.000\end{array}$

Micronutrients and their final concentrations in $\mu g / 1$

$\mathrm{H}_{3} \mathrm{BO}_{3}$

$\mathrm{MnCl}_{2} \cdot 4 \mathrm{H}_{2} \mathrm{O}$

$\mathrm{ZnCl}_{2}$

$\mathrm{CoCl}_{2}$

$\mathrm{CuCl}_{2}$

$\mathrm{Na}_{2} \mathrm{MOO}_{4} \cdot{ }^{2 \mathrm{H}_{2} \mathrm{O}}$

$\mathrm{FeCl}_{3}$

$\mathrm{Na}_{2}$ EDTA. $2 \mathrm{H}_{2} \mathrm{O}$
$185 \cdot 520$

415.447

32.709

0.780

0.009

7.260

96.000

300.000 
while still in the exponential phase of growth to reduce bacterial contaminations. Experiments were done with the algal solutions $3-4$ days after their subculturing.

Stock solutions of PCP and tris buffer were added to a portion of algal cultures and the $\mathrm{pH}$ adjusted with water and $\mathrm{HCl}$ to obtain in a final solution of $1.0 \mathrm{mM}$ concentration of tris and the required concentration of PCP. Three scintillation vials were filled with $4.5 \mathrm{ml}$ of this solution and $0.5 \mathrm{ml}$ of $0.5 \mathrm{micro}$ Curies/ml $\mathrm{NaHC}^{14} \mathrm{O}_{3}$ was added to each. The vials were kept on a rotary shaker under fluorescent light for two hours after which $0.5 \mathrm{ml}$ of $0.1 \mathrm{~N} \mathrm{HCl}$ was added to all vials and air passed through them in order to remove all inorganic $\mathrm{C}^{14} \mathrm{O}_{2}$ remaining in the solution. $10 \mathrm{ml}$ of Aquasol- $2^{R}$ solution (purchased from New England Nuclear Co.) was then added to all vials and subsequently the remaining radioactivity in vials was measured using a Unilux-IIA liquid scintillation counter. One vial out of every three portions was kept in the dark soon after the addition of $\mathrm{NaHC}^{14} \mathrm{O}_{3}$. This vial used as a reference was treated similarly as the other vials. The count rate of this "dark" vial was subtracted from the "light" vial count rate for each data point. This was done in order to correct for any residual radioactivity not associated with the fixation of $\mathrm{C}^{14}$. Quenching correction was found to be negligible. 


\title{
CHAPTER III
}

\section{MEMBRANE TRANSPORT MODELS}

INTRODUCTION

\begin{abstract}
The simplest case of ion transport across membranes can be described in terms of ion adsorption, translocation, and desorption. However, biological studies on cells and subcellular organells indicate that very often chemical processes at the membrane water interface are coupled to the transmembrane translocation of ions and that these processes can limit the flux of ions through the membrane. Studies on artificial lipid membranes, which are simpler systems in comparison with biomembranes have also indicated that for certain biologically active substances the membrane permeable ions are actually formed at the membrane water interface $(9,28,30,52,53)$. It is therefore necessary to develop both theoretical and experimental methods that facilitate a detailed understanding of processes coupled to charge transfer across the membrane. One of the methods is to apply a stepwise electric potential difference across the membrane and to follow the time evolution of the current. The current contains information on the kinetics of charge transport.

In the following sections we present a systematic study of kinetic models of membrane transport which are useful in the analysis of experimental results. Because one of the objectives was to follow the evolution of kinetics from the simplest to more complicated systems, it
\end{abstract}


was considered necessary to incorporate into this study several models that have already been described in the literature. These were (a) models of transport of lipid soluble ions (42), (b) transport of ions facilitated by ion carriers (54), and (c) models applicable to uncouplers of oxidative phosphorylation $(30)$. The objective is to illuminate the relationships between the relaxation parameters, the type of transport mechanism, and the kinetic constants.

The series of kinetic models that will be discussed in this section is depicted in Fig. 13.a-h.

It is assumed that all components of the transport system are initially in equilibrium, and that the perturbation is due to an instantaneous change of transmembrane translocation rate constants, brought about by a stepwise electric potential difference applied across the membrane. The membrane transport system then undergoes a transition towards a new equilibrium state. The objective of the analysis is to predict the time evolution of the transmembrane ion flux, and the final steady state which the system approaches.

Model 1 is the simplest model of transmembrane ion transport. It is applicable to membrane bound ions and has been developed from the studies of redistribution of negatively charged tetraphenylborate and dipicrylamine ions in lipid bilayer membranes (42). The model is applicable to the above real membrane systems on time scale up to $10^{-2}$ - $10^{-1}$ secs. At longer times the existence of interfacial ion transfer invalidates the model.

Model 2 can be considered as a next step in development of more complex models. Model 2 is the model 1 modified by the introduction of a surface reaction at the right hand interface. For transmembrane 


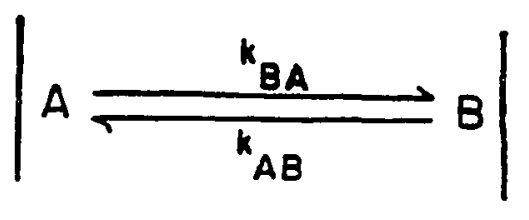

Figure 13.a. MODEL 1.

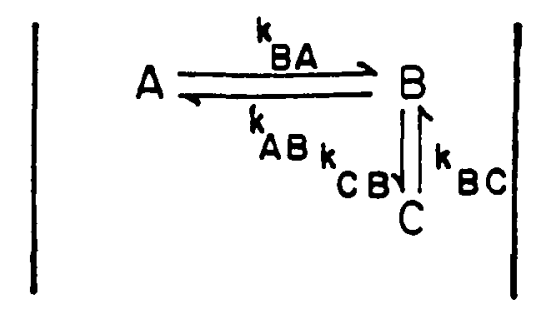

Figure 13.b. MODEL 2 .

transport driven from left to right the surface process functions as a sink. For transmembrane process driven in the opposite direction the surface acts as a reservoir for membrane permeable ions. This model has been introduced purely for the purpose of study of the effect of a simple chemical transformation coupled to the transmembrane translocation.

In model 3 we have added another surface reaction on to the opposite side of the membrane so that there are two surface processes coupled to transmembrane translocation. Such a system contains both the reservoir and the sink of membrane permeable ions.

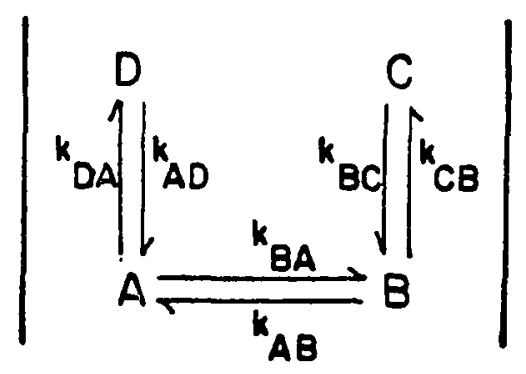

Figure 13.c. MODEL 3. 
Model 4 can be regarded as an extension of model 1 and a special case of model 3. In contrast to model 1 the present model can support a steady state flow of ions across the membrane. It is very likely that it is applicable to the case of transmembrane transport of positively charged lipophilic ions such as tetraphenylarsonium and in general to cases of simple transport when the diffusion of a component forming the membrane permeable ion or the diffusion of the membrane permeable ion itself across the unstirred layers in the aqueous phase does not limit the flux of ions across the membrane.

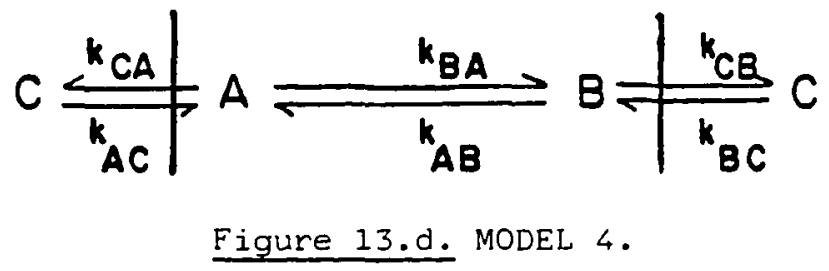

Model 5 plays a prominent role in studies of transmembrane transport. It is assumed that in addition to processes acting as a reservoir and a sink coupled to transmembrane translocation there is a return pathway. This return pathway prevents excessive accumulation of products of transmembrane translocation at the sink side and depletion of components required for the formation of membrane permeable ions at the reservoir side. Due to this feedback mechanism the system operates in cycles and it can sustain a high steady state level of transmembrane flux. If the surface processes are associated with specific interfacial charge transfer reactions, then this model is applicable to several biologically relevant cases, such as ion translocation mediated by some antibiotics $(52,54)$ and transmembrane transfer of hydrogen ions by uncouplers (30). 


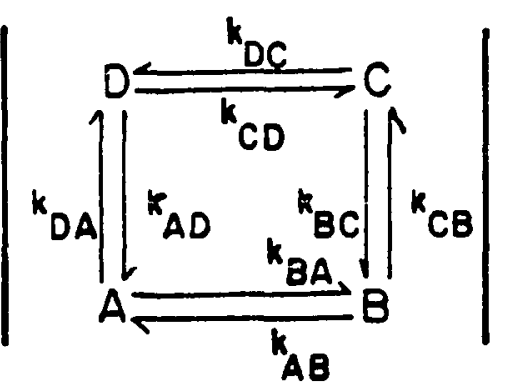

Figure 13.e. MODEL 5 .

Model 6 represents the application of the general model 5 to a special case; action of class I uncouplers in lipid membranes.

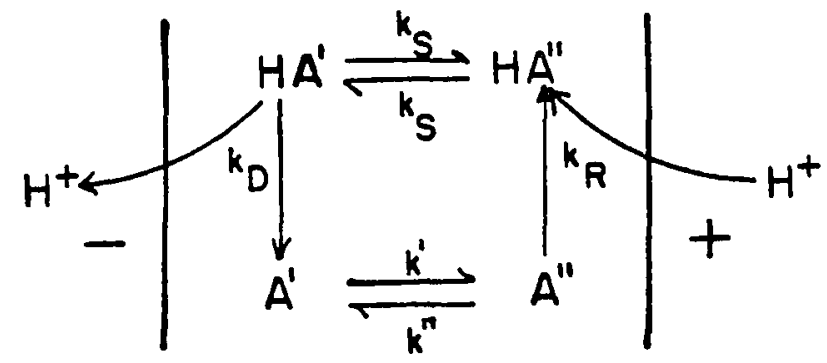

Figure 13.f. MODEL 6 .

The previous models were linear, due to the fact that they are described by linear differential equations. The following ones belong to the category of nonlinear models.

Model 7 has been proposed to explain the action of class II uncouplers in lipid membranes $(8,9,28,30)$. Its nonlinearity originates from the reaction of formation of membrane permeable ions at the surface, which are thought to be dimer complexes $\mathrm{HA}_{2}^{-}$formed by the combination of one neutral (HA) and one negatively charged molecular ion ( $A^{-}$) $(44,45)$. Its complexity arises from the existence of two coupled loops. The biological significance of this model rests in the possibility of coupling of transmembrane flow of negatively charged ions, which are products of surface processes, to the transfer of hydrogen ions from one compartment into another. 


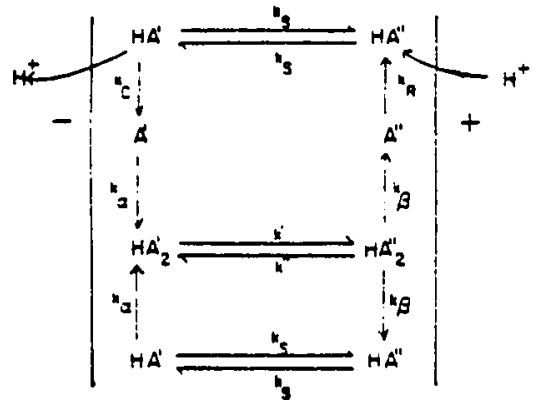

Figure 13.g. MODEL 7: Dimer model A.

Model 8 is of special interest to this project because it includes an alternative pathway of charge transfer across the membrane water interface. In model 7 it was assumed that the charged species crossing the boundary is a proton. This assumption implies that the anion $A^{-}$and the neutral molecule $H A$ are favorably oriented at the interface so that such a transfer is possible. In contrast, in the present model we assume that the proton association and dissociation takes place on the aqueous side of the interface, and that the net transfer of charge across the membrane is associated with the interfacial transfer of $H A$ and $A^{-}$molecules.

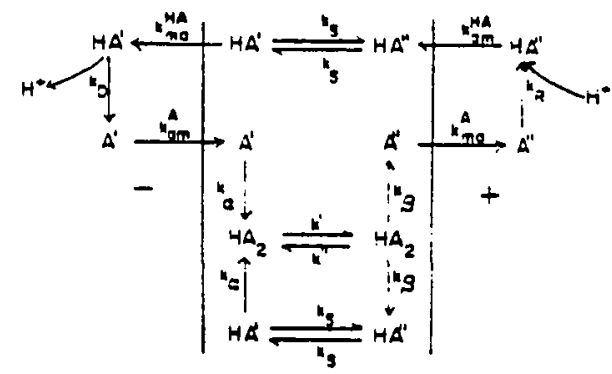

Figure 13.h. MODEL 8: Dimer model B.

It is expected that kinetic analysis will provide us with criteria for distinguishing between the alternative models. This study is relevant because of the existence of pesticide induced transfer of electric charge in lipid membranes. 
MODEL 1: RELAXATION DUE TO ION REDISTRIBUTION IN MEMBRANES

We have shown in Chapter I that electrodiffusion of ions in membranes and specifically transmembrane ion flux can be given by equations of chemical kinetics. In this section we illustrate the kinetic concept on the case of simplest type of membrane transport, model 1 (Fig. 14). It is described by two coupled differential equations

$$
\begin{aligned}
& d n_{A} / d t=-k_{B A} n_{A}+k_{A B} n_{B}, \\
& d n_{B} / d t=k_{B A} n_{A}-k_{A B} n_{B}
\end{aligned}
$$

where $k^{\prime} s$ are the voltage dependent rate constants of transmembrane translocation and n's are the densities of ions per unit area of membrane surface.

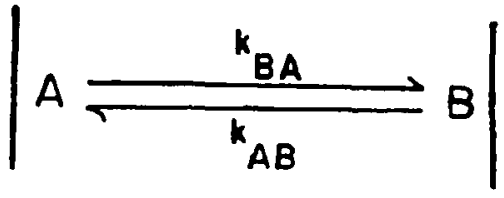

Figure 14. MODEL 1: Ion redistribution in membranes.

It follows that

$$
d n_{A} / d t+d n_{B} / d t=\frac{d}{d t}\left(n_{A}+n_{B}\right)=0
$$

which means that

$$
n_{A}+n_{B}=\text { constant }=N_{O^{*}}
$$

This is a statement of the fact that total number of membrane bound ions is conserved. At initial equilibrium, at time $t<0$, and at the final steady state, the system is in equilibrium, i.e., $d n_{A} / d t=0$ and $d n_{B} / d t=0$. The initial distribution of species $A$ and $B$ is 


$$
\mathrm{n}_{\mathrm{A}}^{\text {in }}=\mathrm{N}_{0} \mathrm{k}_{\mathrm{AB}} /\left(\mathrm{k}_{\mathrm{AB}}+\mathrm{k}_{\mathrm{BA}}\right)
$$

and $\quad n_{B}^{i n}=N_{0} k_{B A} /\left(k_{A B}+k_{B A}\right)$

Initially, when the voltage applied across the membrane is zero,

$$
k_{A B}^{\text {in }}=k_{B A}^{\text {in }}=k_{0} \text {. }
$$

Therefore the initial concentrations are

$$
\mathrm{n}_{\mathrm{A}}^{\text {in }}=\mathrm{n}_{\mathrm{B}}^{\text {in }}=\mathrm{N}_{0} / 2
$$

Due to the electric potential difference applied at $t \geq 0$ the transmembrane rate constants change according to Eqs. 57 and 58 (Eqs. 13,14).

$$
\begin{aligned}
& \mathrm{k}_{B A}=k_{0} \cdot \exp (\mathrm{eV} / 2 \mathrm{kT}) . \\
& k_{A B}=k_{0} \cdot \exp (-\mathrm{eV} / 2 \mathrm{kT}) .
\end{aligned}
$$

The result is the net flow of ions from A to $B$. The flux of ions across the membrane is given by

$$
j(t)=k_{B A} \cdot n_{A}-k_{A B} \cdot n_{B} \cdot
$$

The variation of $n_{A}$ with time is obtained by solving Eq. 51 using the relation 54 . This gives

$$
d n_{A} / d t=-\left(k_{B A}+k_{A B}\right) n_{A}+k_{A B} N_{0}
$$

Integrating this equation between the times $t=0$ and $t=t$ we get $n_{A}(t)$ in the form

$$
n_{A}(t)=L \cdot \exp (-t / \tau)+n_{A}(\infty),
$$


where $L=\left(N_{O} / 2\right) \cdot\left(k_{B A}-k_{A B}\right) /\left(k_{B A}+k_{A B}\right)$,

and $\tau=\left(k_{B A}+k_{A B}\right)^{-1}$,

and $n_{A}(\infty)$ is the steady state concentration of $A$ given by the Eq. 55 . The surface density of ions on the opposite side of the membrane is obtained similarly.

$$
n_{B}(t)=-L, \exp (-t / \tau)+n_{B}(\infty)
$$

where $n_{B}(\infty)$ is the steady state density of $B$, and is given by Eq. 56 . Then the transmembrane flux given by Eq. 59 becomes

$$
j(t)=j_{0} \cdot \exp (-t / \tau),
$$

where $j_{0}$ is the initial flux equal to

$$
j_{0}=\left(N_{0} / 2\right) \cdot\left(k_{B A}-k_{A B}\right)^{2} /\left(k_{B A}+k_{A B}\right) .
$$

The transport system does not support a steady state flux. The total amount of charge $Q$ that crosses the membrane for a given applied voltage is obtained by integrating Eq. 65 . The result is

$$
\begin{aligned}
Q & =j_{0} \cdot \tau \\
& =\left(k_{B A}-k_{A B}\right)^{2} /\left(k_{B A}+k_{A B}\right)^{2} \cdot\left(N_{0} / 2\right) \\
& =\tanh ^{2}(e V / 2 k T) \cdot N_{0} / 2 .
\end{aligned}
$$

This expression shows that by the application of a sufficiently high voltage almost all of the ions adsorbed at one membrane water interface can be transferred to the other side. Hence it may be used to study the adsorption of such ions on membranes.

Both the initial membrane flux and the relaxation time constant 
are voltage dependent. The initial flux increases with the applied voltage according to

$$
j_{0}=k_{0} N_{0} \cdot \tanh (e V / 2 k T) \cdot \sinh (e V / 2 k T),
$$

and the relaxation time constant decreases according to

$$
\begin{aligned}
\tau & =\left(k_{B A}+k_{A B}\right)^{-1} \\
& =\left(2 k_{0} \cdot \cosh (e V / 2 k T)\right)^{-1} .
\end{aligned}
$$

If should be noted that due to the existence of reverse flow from $B$ to $A,\left(k_{A B} \neq 0\right)$ the relaxation time constant is reduced. In the absence of reverse translocation $\tau=k_{B A}^{-1}$ whereas with the possibility of return flow $\tau=\left(k_{B A}+k_{A B}\right) \cdot{ }^{-1}$ Thus it is to be expected that in more complicated transport schemes the relaxation time constant will become smaller due to pathways resulting in return flow, or resulting in regeneration of membrane permeable species on the side of membrane with depleted population of membrane permeable ions.

MODEL 2: TRANSMEMBRANE TRANSLOCATION COUPLED TO

A SINGLE SURFACE REACTION

\begin{abstract}
This model has been considered in order to study the effect of surface process and its location with respect to the direction of transmembrane translocation on characteristics of transient membrane flux. The model is shown in Fig. 15. Component $C$ can be either regarded as a sink for membrane permeable species, if the transport is driven from $A$ to $B$, or as a reservoir for transport driven in the opposite direction.
\end{abstract}




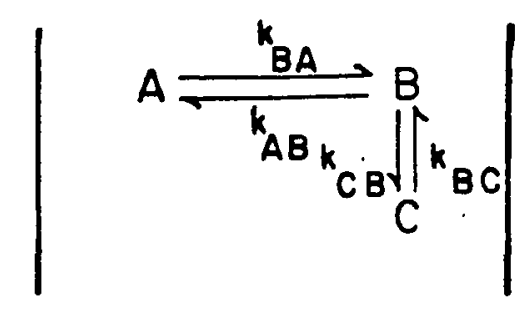

Figure 15. MODEL 2: Transmembrane translocation coupled to a single surface reaction.

The transport model is described by the following set of equations:

$$
\begin{aligned}
& d n_{A} / d t=-k_{B A} n_{A}+k_{A B} n_{B} \cdot \\
& d n_{B} / d t=k_{B A} n_{A}-\left(k_{A B}+k_{C B}\right) n_{B}+k_{B C} n_{C} \cdot \\
& d n_{C} / d t=k_{C B} n_{B}-k_{B C} n_{C} .
\end{aligned}
$$

The transmembrane flux is given either by

$$
j(t)=k_{B A} n_{A}-k_{A B} n_{B}
$$

for the direction of transport from $A$ to $B$, ( $A B C$ case), or by

$$
j(t)=k_{A B} n_{B}-k_{B A} n_{A} \text {, }
$$

for the net flow direction from $B$ to $A$, (CBA case).

The general solution of the system, applicable to both directions of flow is of the form

$$
j(t)=j_{01} \cdot \exp \left(-t / \tau_{1}\right)+j_{02} \cdot \exp \left(-t / \tau_{2}\right) \cdot
$$

We find that the reciprocal of the shorter relaxation time constant $\tau_{1}$ is equal to 


$$
\begin{aligned}
& \tau_{1}^{-1}=\frac{1}{2} \cdot\left[k_{B A}+k_{A B}+k_{C B}+k_{B C}\right. \\
& \left.\quad-\left\{\left(k_{B A}+k_{A B}-k_{C B}-k_{B C}\right)^{2}+4 k_{A B} k_{C B}\right\}^{\frac{1}{2}}\right],
\end{aligned}
$$

and the reciprocal of the longer relaxation time constant $\tau_{2}$ is

$$
\begin{aligned}
\tau_{2}^{-1}=\frac{1}{2} \cdot\left[k_{B A}+k_{A B}+k_{C B}+k_{B C}\right. \\
\\
\left.\quad-\left\{\left(k_{B A}+k_{A B}-k_{C B}-k_{B C}\right)^{2}+4 k_{A B} k_{C B}\right\}^{\frac{1}{2}}\right] .
\end{aligned}
$$

From these expressions it is obvious that even for simple schemes of membiane transport one cannot simply identify individual relaxation time constants with individual processes. Some insight, however can be gained by considering limiting conditions, such as slow and fast kinetics of surface reaction.

(a) Slow Surface Kinetics, $k_{C B}+k_{B C}<k_{B A}+k_{A B}$.

In this approximation the reciprocal time constants become

$$
\tau_{1}^{-1}=k_{B A}+k_{A B}+k_{A B} k_{C B} /\left(k_{B A}+k_{A B}\right)
$$

and $\quad \tau_{2}^{-1}=k_{C B}+k_{B C}-k_{A B} k_{C B} /\left(k_{B A}+k_{A B}\right)$

In this case the short relaxation time constant $\tau_{1}$ is primarily determined by the rate constants of transmembrane translocation. The kinetic constants of the surface process are contained in a correction term. The long relaxation time constant $\tau_{2}$ is determined mainly by the kinetic constants of the surface process. In this case the translocation 
rate constants are present only in a correction term. Because only the rate constants of transmembrane translocation are voltage dependent, we can in principle differentiate between the two relaxation processes. The question of whether the fast or slow component of the relaxation process can be measured depends on the magnitudes of the amplitudes $j_{01}$ and $j_{02}$. Favorable conditions for the experimental determinations correspond to $\tau_{2} / \tau_{1}>2$ and $j_{02} / j_{01} \leq 1$. In such cases the transition from the initial fast relaxation to the slower one can be well resolved.

The relaxation characteristics of the model for the parameters corresponding to conditions of slow surface kinetics are shown in Fig. 16, where we plot the voltage dependencies of relaxation time constants and flux component amplitudes. The figure shows that the fast component dominates the transmembrane flux. This component can be associated with transmembrane translocation kinetics, its amplitude increases and the relaxation time constant decreases with the applied voltage. The slow component of the transmembrane flux, which is due to the surface kinetics, is either independent of voltage or only weakly voltage dependent.

The results in Fig. 16 also indicate that the contribution of the slow flux component depends on the direction of the net flux. If chemical constituent $C$ of transport scheme acts as a sink ( $A B C$ case), the amplitude of the slow flux diminishes with the applied voltage whereas if the constituent $\mathrm{C}$ acts as a reservoir (CBA case) the amplitude of the slow flux approaches a limit at high applied voltage.

(b) Fast Surface Kinetics; $k_{C B}+k_{B C}>k_{B A}+k_{A B}$ Due to the voltage dependence of transmembrane kinetics the 

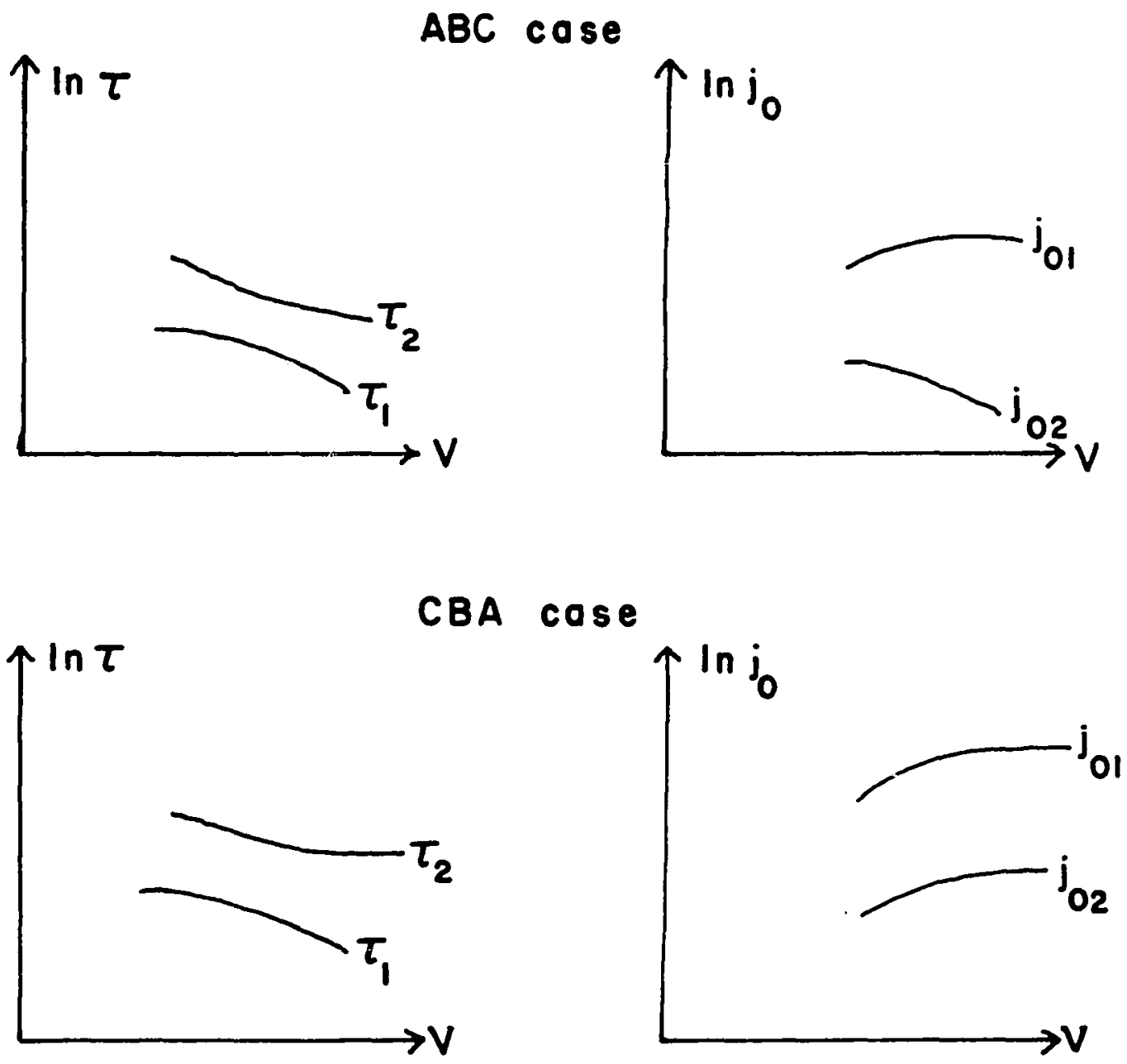

Figure 16. MODEL 2: SLOW SURFACE REACTION. Variation of the time constant and the flux amplitude with the voltage for model 2 when the surface reaction is slow; in the ABC-case the component $C$ acts as $a$ sink and in the CBA-case the component $\mathrm{C}$ acts as a source of membrane permeable ions. The slow time constant $\tau_{2}$ is weakly voltage dependent in both cases (Eq. 79). 
condition of fast surface kinetics is valid only at sufficiently low voltage where the condition $k_{C B}+k_{B C}>k_{B A}+k_{A B}$ is satisfied. In this approximation the reciprocal time constants are equal to

$$
\tau_{1}^{-1}=k_{C B}+k_{B C}+k_{A B} k_{B C} /\left(k_{C B}+k_{B C}\right)
$$

and

$$
\tau_{2}^{-1}=k_{B A}+k_{A B}-k_{A B} k_{B C} /\left(k_{C B}+k_{B C}\right)
$$

In contrast to slow surface kinetics, we find that the short relaxation time constant $\tau_{1}$ is primarily determined by the surface process. The translocation kinetics represented by the rate constant of transition from $B$ to $A$, is present only in a correction term. For this reason the voltage dependence of $\tau_{1}$ is closely related to the direction of net flow. If the surface process acts as a sink ( $A B C$ case) $k_{A B}$ decreases with the applied voltage and the time constant $\tau_{1}$ remains essentially voltage independent. If we consider the surface as a reservoir for membrane permeable ions ( $C B A$ case), the rate constant $k_{A B}$ increases with the applied voltage and the time constant $\tau_{1}$ decreases.

In the case of fast surface kinetics the time constant of slow relaxation process is primarily determined by the translocation kinetics and is therefore strongly voltage dependent.

Voltage dependences of the relaxation time constants and flux amplitudes are shown in Fig. 17. Two features of the results are worthy of mentioning. First, in the case of fast surface kinetics the transmembrane flux is dominated by the slow component, a finding opposite to that established for the case of slow surface kinetics. Second, as the voltage is increased the transmembrane kinetics become faster and therefore above a certain voltage the system underyues a transition from a fast surface kinetics regime to a slow surface kinetics regime. 

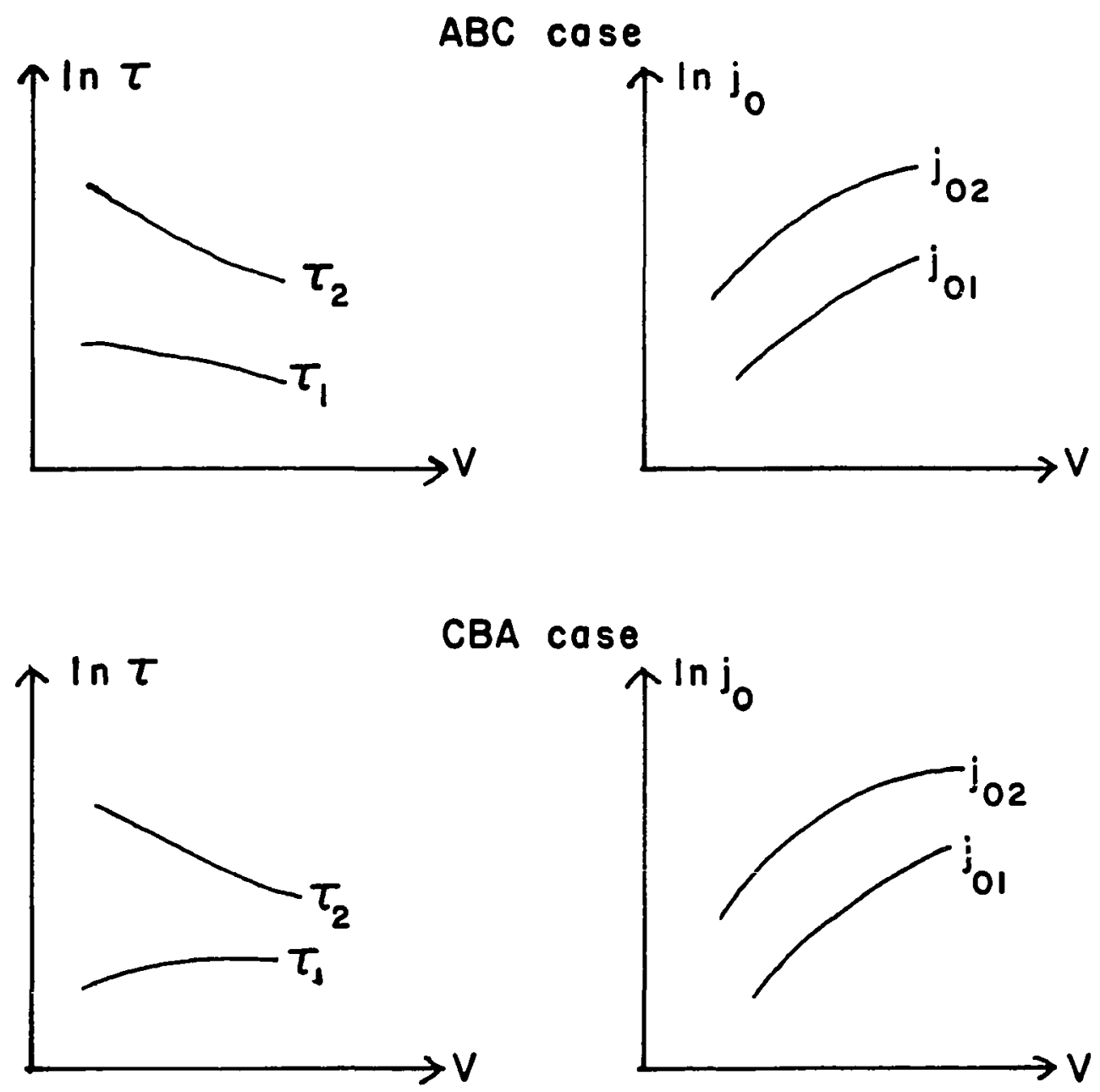

CBA case

Figure 17. MODEL 2: FAST SURFACE REACTION. Variation of the time constant and the flux amplitude with the voltage for model 2 when the surface reaction is fast; in the ABC-case the component $C$ acts as $a$ sink and in the CBA-case the component $C$ acts as a source of membrane permeable ions. The fast time constant $\tau$, is weakly voltage dependent (Eq. 80) in contrast to the time constant of the slow process, which is strongly voltage dependent. 
MODEL 3: TRANSMEMBRANE TRANSLOCATION COUPLED TO TWO IDENTICAL SURFACE REACTIONS

The model studied in this section is shown in Fig. 18. Reaction A - B represents ion translocation across the membrane; processes D - A and B - C symbolize surface reactions which are coupled to ion translocation. These surface reactions are responsible for the generation and depletion of membrane permeable ions $A$ and $B$.

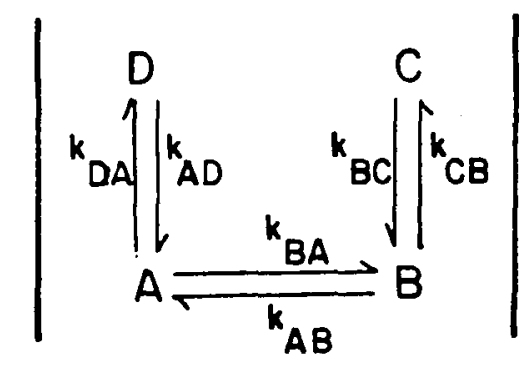

Figure 18. MODEL 3. Transmembrane translocation coupled to two identical surface reactions.

The model is described by the following set of differential equations.

$$
\begin{aligned}
& d n_{A} / d t=-\left(k_{B A}+k_{D A}\right) n_{A}+k_{A B} n_{B}+k_{A D} n_{D} \\
& d n_{B} / d t=k_{B A} n_{A}-\left(k_{A B}+k_{C B}\right) n_{B}+k_{B C} n_{C} \\
& d n_{C} / d t=k_{C B} n_{B}-k_{B C} n_{C} \\
& d n_{D} / d t=k_{D A} n_{A}-k_{A D} n_{D} .
\end{aligned}
$$

If we assume that the system is symmetrical then the kinetic constants of processes $D-A$ and $B-C$ become identical; i.e. $k_{D A}=k_{C B}$ and $k_{A D}=$ $k_{B C}$. Defining the density desplacements $x_{\alpha}$ from the inital equilibrium by the Eqs. 


$$
\begin{aligned}
& n_{A}=n_{A}^{i n}-x_{A}, \\
& n_{B}=n_{B}^{i n}-x_{B}, \\
& n_{C}=n_{C}^{i n}-x_{B}, \\
& n_{D}=n_{D}^{i n}-x_{D},
\end{aligned}
$$

it can be shown that due to the symmetry of the kinetic scheme

$$
x_{A}=-x_{B} \text { and } x_{C}=-x_{D}
$$

at all times. Furthermore, the initial surface concentrations are also symmetric

$$
n_{A}^{\text {in }}=n_{B}^{\text {in }} \text { and } n_{C}^{\text {in }}=n_{D}^{\text {in }} \text {. }
$$

Using symmetry properties the system of Eqs. 82-85 can be reduced to two:

$$
d x_{A} / d t=-\left(k_{B A}+k_{A B}+k_{C B}\right) x_{A}+k_{B C} x_{C}
$$

and

$$
d x_{C} / d t=k_{C B} x_{A}-k_{B C} x_{C}
$$

The general solution of this system is of the form

$$
x_{\alpha}=\sum_{j=1}^{2} a, j=\exp \left(-\lambda_{i} \cdot t\right)+\text { const. }_{\alpha},(\alpha=A, C)
$$

where const. ${ }_{\alpha}$ are the density displacements at the steady state from the initial equilibrium densities. $a_{\alpha, j}$ are the density displacement amplitudes obtained from the condition of initial equilibrium; i.e. $x=$ 0 at $t=0$.

We find that the transmembrane flux defined by 


$$
j(t)=k_{B A} n_{A}(t)-k_{A B} n_{B}(t)
$$

has two components of the form

$$
j(t)=j_{01} \cdot \exp \left(-t / \tau_{1}\right)+j_{02} \cdot \exp \left(-t / \tau_{2}\right),
$$

where

$$
j_{01}=\frac{N_{0}\left(k_{B A}-k_{A B}\right) \cdot \lambda_{2} \cdot\left(\lambda_{1}-k_{B C}\right)}{2\left(k_{C B}+k_{B C}\right) \cdot\left(\lambda_{1}-\lambda_{2}\right)}
$$

and

$$
j_{02}=\frac{N_{0}\left(k_{B A}-k_{A B}\right) \cdot \lambda_{1} \cdot\left(k_{B C}-\lambda_{2}\right)}{2\left(k_{C B}+k_{B C}\right) \cdot\left(\lambda_{1}-\lambda_{2}\right)},
$$

where $\mathrm{N}_{0}$ is the total density of the transport scheme constituents,

$$
\mathrm{N}_{0}=\mathrm{n}_{\mathrm{A}}+\mathrm{n}_{\mathrm{B}}+\mathrm{n}_{\mathrm{C}}+\mathrm{n}_{\mathrm{D}} \text {. }
$$

The reciprocal relaxation time constants are as follows. For the fast process we find

$$
\begin{aligned}
& 1 / \tau_{1}=\lambda_{1}=\frac{1}{2}\left[k_{B A}+k_{A B}+k_{C B}+k_{B C}\right. \\
& \left.+\left\{\left(k_{B A}+k_{A B}\right)^{2}+\left(k_{C B}+k_{B C}\right)^{2}+2\left(k_{B A}+k_{A B}\right)\left(k_{C B}-k_{B C}\right)\right\}^{\frac{1}{2}}\right],
\end{aligned}
$$

and for the slow process

$$
\begin{aligned}
& 1 / \tau_{2}=\lambda_{2}=\frac{1}{2}\left[k_{B A}+k_{A B}+k_{C B}+k_{B C}\right. \\
& \left.-\left\{\left(k_{B A}+k_{A B}\right)^{2}+\left(k_{C B}+k_{B C}\right)^{2}+2\left(k_{B A}+k_{A B}\right)\left(k_{C B}-k_{B C}\right)\right\}^{\frac{1}{2}}\right] .
\end{aligned}
$$

Both the flux component amplitudes, $j_{01}$ and $j_{02}$, and the relaxation time constants are dependent on the voltage via the voltage dependent translocation rate constants $k_{B A}$ and $k_{A B}$. 
In order to gain some insight into the behavior of this kinetic model we discuss separately the case of slow and fast surface kinetics.

(a) Slow Surface kinetics, $k_{C B}+k_{B C}<<k_{B A}+k_{A B}$.

Since at low voltages the transmembrane kinetics are slow, the condition of slow surface kinetics may in some instances be valid only at sufficiently high voltages. It follows from Eqs. 95 and 96 that in this approximation the reciprocal time constant of the fast relaxation becomes

$1 / \tau_{1}=\lambda_{1}=k_{B A}+k_{A B}+k_{C B}+\frac{1}{4}\left(k_{C B}+k_{B C}\right)^{2} /\left(k_{B A}+k_{A B}\right)$,

and the reciprocal time constant of the slow relaxation becomes

$1 / \tau_{2}=\lambda_{2}=k_{B C}-\frac{1}{4}\left(k_{C B}+k_{B C}\right)^{2} /\left(k_{B A}+k_{A B}\right)$.

The relaxation time constant of the fast component is primarily determined by the kinetic constants of the transmembrane translocation, whereas for the time constant of the slow component we find that it is dominated by the rate constant of formation of membrane permeable ions. It also follows from Eqs. 97 and 98 that the time constant of the fast process is strongly voltage dependent, whereas the time constant of the slow component is weakly voltage dependent.

In this approximation the flux component amplitude of the fast component is

$$
j_{01}=\left(N_{0} / 2\right)\left(k_{B A}-k_{A B}\right) \cdot k_{s}^{e q} /\left(1+k_{s}^{e q}\right)
$$

and that of the slow component is 


$$
j_{02}=\left(N_{0} / 8\right) \frac{k_{C B}+k_{B C}}{k_{B A}+k_{A B}}\left(k_{B A}-k_{A B}\right),
$$

where $\mathrm{k}_{\mathrm{S}}^{\text {eq }}=\mathrm{k}_{\mathrm{BC}} / \mathrm{k}_{\mathrm{CB}}$ is the equilibrium constant of formation of membrane permeable ions, $n_{B}^{e q}=k_{S}^{e q} n_{C}^{e q}$ and $n_{A}^{e q}=k_{S}^{e q} n_{D}^{e q}$. These expressions indicate that for the case of fast surface kinetics the fast relaxation process dominates the transient flux in the short time domain.

(b) Fast Surface Kinetics, $k_{C B}+k_{B C}>>k_{B A}+k_{A B}$.

As mentioned before, the condition of fast surface kinetics may be valid only at sufficiently low voltages. In this approximation the reciprocal time constants are given by the following equations. For the fast relaxation

$$
1 / \tau_{1}=\lambda_{1}=\left(k_{C B}+k_{B C}\right)\left(1+\frac{1}{1+k_{s}^{e q}} \cdot \frac{k_{B A}+k_{A B}}{k_{C B}+k_{B C}}\right),
$$

and for the slow relaxation

$$
1 / \tau_{2}=\lambda_{2}=\left(k_{B A}+k_{A B}\right) \frac{k_{s}^{e q}}{1+k_{s}^{e q}} .
$$

These expressions indicate that in the case of fast surface kinetics the fast relaxation process is dominated by the kinetics of the surface reaction, whose main feature is that it is voltage independent. The slow process can be associated with the redistribution of ions across the membrane. The slow relaxation time constant is therefore strongly voltage dependent. The corresponding flux amplitudes are as follows: 
$j_{01}=\left(N_{0} / 2\right) \frac{k_{s}^{e q}}{\left(k_{s}^{e q}+1\right)^{2}} \frac{k_{B A}+k_{A B}}{k_{C B}+k_{B C}}\left(1+\frac{k_{B A}+k_{A B}}{k_{B C}+k_{C B}}\right)\left(k_{B A}-k_{A B}\right)$,

and

$j_{02}=\left(N_{0} / 2\right) \frac{k_{s}^{e q}}{k_{s}^{e q}+1}\left(1-\frac{k_{B A}+k_{A B}}{k_{B C}+k_{C B}}\right)\left(k_{B A}-k_{A B}\right)$.

These results indicate that in the case of fast surface kinetics the net flux is dominated by the slow component, but that the amplitude of the fast component increases with the applied voltage more rapidly than the amplitude of the slow one. This is an interesting feature that can be used for kinetic discrimination. It corresponds to the development of a fast relaxation component with the applied voltage. At sufficiently high voltage a crossover can be reached (see Fig. 19) above which the transmembrane flux becomes dominated by the fast component. The crossover corresponds to the transition from the regime of fast surface kinetics to that of slow surface kinetics.

The results also indicate how the voltage dependence of the relaxation time constants and flux amplitudes can be holpful in discriminating between various kinetic regimes. To illustrate this point we plot in Fig. 19 the quantities of interest that were obtained from the solution of the model.

Below we summarize the main features of the present kinetic scheme of two identical surface reactions coupled to transmemurane translocation.

(a) Case of slow surface kinetics

1a. The flux amplitude associated with the fast relaxation process is greater than the flux amplitude of the slow process. 

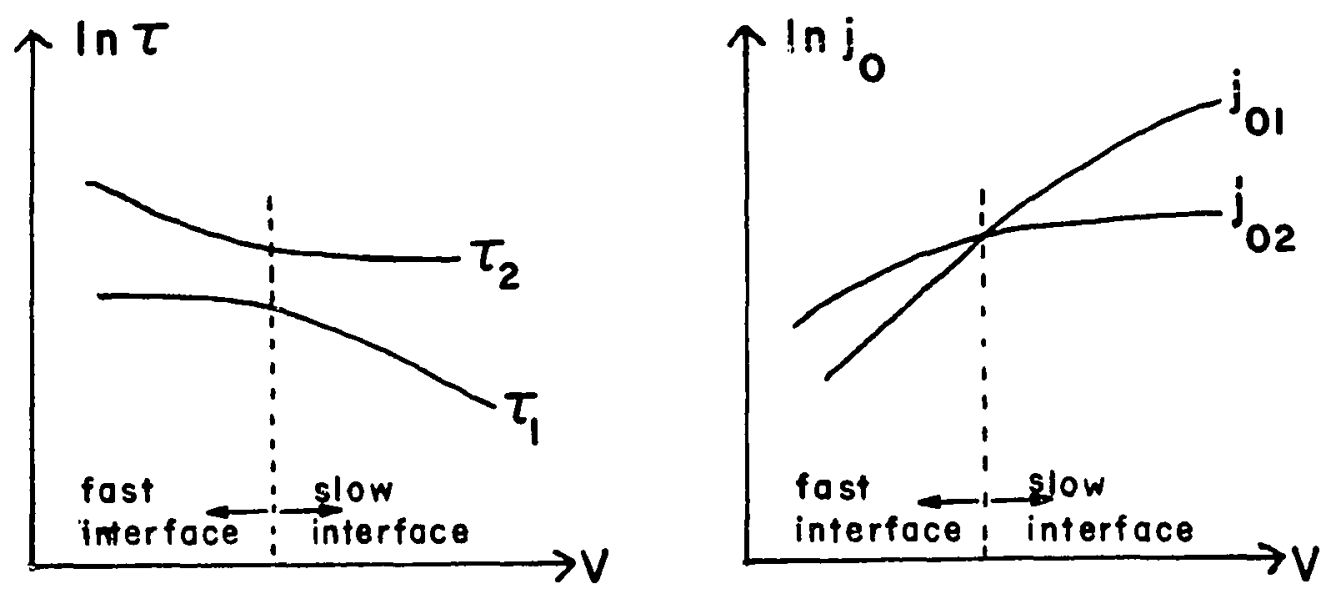

Figure 19. MODEL 3: Variation of the time constants and the flux amplitudes for model 3 with voltage for two kinetic regimes. When the interfacial reaction is fast, the slow transient dominates and the fast relaxation time constant is voltage independent. When the interfacial reaction is slow, the fast transient dominates and the slow time constant and the slow flux amplitude are voltage independent.

2a. The flux amplitude of fast process increases with the applied voltage as $\sinh (\mathrm{eV} / 2 \mathrm{kT})$, whereas the flux amplitude of slow process remains approximately voltage independent at $\mathrm{V}>4 \mathrm{kT} / \mathrm{e}$.

3a. The time constant of the fast relaxation process rapidly decreases with the voltage applied, whereas the time constant of the slow process is approximately voltage independent.

(b) Case of fast surface kinetics

1b. The flux amplitude associated with the slow process dominates the transient flux.

2b. The flux amplitude of the fast process increases more rapidly with the applied voltage than the amplitude of the slow process. 
3b. The time constant of the fast process is weakly voltage dependent.

The above properties of fast surface kinetics hold at low voltages, but as the relaxation amplitude of the fast and slow processes become comparable, the time constant of the fast process begins to decrease rapidly with the applied voltage. In the voltage range where the slow component of flux dominates (i.e., at low voltages) the time constant of the slow process is strongly voltage sensitive, but the rate of decrease diminishes at high applied voltages. This change in voltage dependence corresponds to transition from the regime of fast surface kinetics to the regime of slow surface kinetics.

MODEL 4: TRANSMEMBRANE TRANSLOCATION COUPLED TO TWO INTERFACIAL TRANSFER REACTIONS

The transport scheme discussed in this section is depicted in Fig. 20. It is in certain aspects similar to the previous model. Model 4 consists of four elementary reaction steps symmetrically coupled to the transmembrane translocation of ions. It differs from model 3 in the assumption of the form of the reactions coupled to the transmembrane translocation. Transformation A - B can be regarded again as ion translocation across the membrane, and the reactions $C-A$ and $B-C$ are assumed to represent charge transfer across the membrane water interface. Due to the presence of these interfacial charge transfer processes this scheme predicts existence of a steady state flow. For the purpose of greater simplicity, it is assumed that the bulk concentration of species $C\left(=c_{C}\right)$ is constant. 


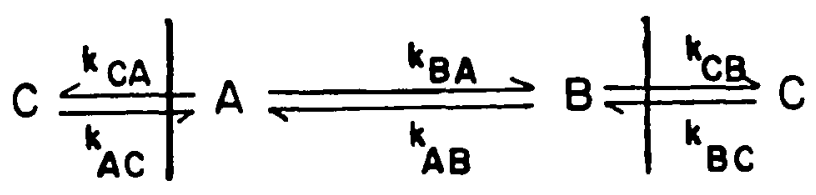

Figure 20. MODEL 4: Transmembrane translocation coupled to two interfacial transfer reactions.

The rates of change of surface densities of species $A$ and $B$ are given by the differential equations

$$
d n_{A} / d t=-\left(k_{B A}+k_{C A}\right) n_{A}+k_{A B} n_{B}+k_{A C} c_{C}
$$

and

$$
d n_{B} / d t=k_{B A} n_{A}-\left(k_{A B}+k_{C B}\right) n_{B}+k_{B C} c_{C}
$$

Therefore, it follows that the general solution has the following form

$$
\begin{aligned}
& n_{A}(t)=a_{A 1} \exp \left(-\lambda_{1} t\right)+a_{A 2} \exp \left(-\lambda_{2} t\right)+\bar{n}_{A}, \\
& n_{B}(t)=a_{B 1} \exp \left(-\lambda_{1} t\right)+a_{B 2} \exp \left(-\lambda_{2} t\right)+\bar{n}_{B},
\end{aligned}
$$

where $\bar{n}_{A}$ and $\bar{n}_{B}$ are steady state concentrations of $A$ and $B$. This shows that the transmembrane flux, in contrast to the previous model, has three components

$$
j(t)=j_{01} \exp \left(-\lambda_{1} t\right)+j_{02} \exp \left(-\lambda_{2} t\right)+\bar{j}
$$

where $\quad \bar{j}=k_{B A} \bar{n}_{A}-k_{A B} \bar{n}_{B}$

is the steady state flux across the membrane.

Due to the simplicity of the model the mathematics is very transparent, so that this model can be used to illustrate the concept of kinetic limitation of membrane transport and the manifestation of the presence of kinetic limitation in the voltage dependence of steady 
state flux and in the transient characteristics.

The density amplitudes $a_{A j}$ and $a_{B j}$, and the reciprocal relaxation time constants $\lambda_{j}$ can be obtained in the usual manner from the following two Eqs.

$$
\begin{aligned}
& \left\{-\left(k_{B A}+k_{C A}\right)+\lambda_{j}\right\} a_{A j}+k_{A B} a_{B j}=0, \\
& k_{B A} a_{A j}+\left\{-\left(k_{A B}+k_{C B}\right)+\lambda_{j}\right\} a_{B j}=0,
\end{aligned}
$$

and two initial densities $n_{A}^{i n}$ and $n_{B}^{i n}$.

$$
\text { It is important to note that the reciprocal relaxation time }
$$

constants, obtained by solving the characteristic Eq.

$\operatorname{det}\left(\begin{array}{cc}\lambda-\left(k_{B A}+k_{C A}\right) & k_{A B} \\ k_{B A} & \lambda-\left(k_{A B}+k_{C B}\right)\end{array}\right)=0$

do not contain kinetic constants $k_{A C}$ and $k_{B C}$. Thus the relaxation time constants are insensitive to the kinetic parameters of transfer from the aqueous medium into the membrane. This is because the concentration of species $C$, which is present in the aqueous phase, does not change with time.

As the next step we examine the properties of the steady state flux. It can be obtained by equating Eqs. 105 and 106 to zero and using definition 109.

$$
\bar{j}=c_{C} \cdot \frac{\frac{k_{A C}}{k_{C A}} k_{B A}-\frac{k_{B C}}{k_{C B}} k_{A B}}{1+\frac{k_{B A}}{k_{C A}}+\frac{k_{A B}}{k_{C B}}} .
$$


This expression contains group of terms pertinent to the left and right membrane surfaces. If due to sufficiently high applied voltage the terms containing $\mathrm{k}_{\mathrm{AB}}$ can be made insignificant, (see Eqs. 120) it becomes obvious that the limiting values of the flux from $A$ to $B$ is either $c_{C} \frac{k_{A C}}{k_{C A}} k_{B A}$ if $k_{B A}<<k_{C A}$, or $c_{C} k_{A C}$ if $k_{B A}>>k_{C A}$. In the former case the flow is limited by the transformation $\dot{A}-B$, i.e. by translocation across the membrane. This means that under such circumstances the membrane potential energy barrier limits the fiux of ions across the membrane. In the latter case, i.e. when $\bar{j}=c_{C}{ }_{A C}$, the transmembrane flux is limited by the slow charge transfer across the membrane water interface.

In the following sections we illustrate the remaining features of this model by presenting a complete analysis of this kinetic scheme for the case of symmetric system.

A Subcase of Symmetric Transport Scheme

It will be assumed that the kinetic rate constants of interfacial processes are symmetric, i.e. $k_{A C}=k_{B C}=k_{\text {in }}$ and $k_{C A}=k_{C B}=$ $\mathrm{k}_{\text {out }}$ In this case the characteristic equation yields two roots. For the fast component of the transient flux we find that the reciprocal relaxation time constant is given by

$$
1 / \tau_{1}=\lambda_{1}=k_{B A}+k_{A B}+k_{\text {out }}
$$

and for the slow process,

$$
1 / \tau_{2}=\lambda_{2}=k_{\text {out }}
$$

However, due to the symmetry of the transport scheme the amplitudes 
associated with the slow process $a_{A 2}$ and $a_{B 2}$ become equal to zero. Thus, the symmetric transport system has only a single transient and a steady state component

$$
j(t)=j_{01} \exp \left(-\lambda_{1} t\right)+\bar{j}
$$

The amplitude of the transient flux and the steady state flux are closely related to each other,

$j_{01}=\frac{k_{\text {in }}}{k_{\text {out }}} c_{C} \frac{k_{B A}+k_{A B}}{k_{\text {out }}} \frac{k_{B A}-k_{A B}}{1+\left(k_{B A}+k_{A B}\right) / k_{\text {out }}}$,

and

$$
\bar{j}=\frac{k_{\text {in }}}{k_{\text {out }}} c_{C} \frac{k_{B A}-k_{A B}}{1+\left(k_{B A}+k_{A B}\right) / k_{\text {out }}}
$$

Their ratio is

$$
\frac{j_{01}}{\bar{j}}=\frac{k_{B A}+k_{A B}}{k_{\text {out }}} \text {. }
$$

This expression shows that the transient current becomes significant, as compared to the steady state, only if the transmembrane transport becomes limited by the slow interfacial kinetics, i.e., $\left(k_{\mathrm{AB}}+\right.$ $\left.\mathrm{k}_{\mathrm{BA}}\right) / \mathrm{k}_{\text {out }} \geq 1$

The voltage dependence of translocation rate constants are given by (Eqs. 13,14)

$$
k_{B A}=k_{0} \exp (e V / 2 k T),
$$

and

$$
k_{A B}=k_{0} \exp (-e V / 2 k T) .
$$

Then the ratio of the flux components becomes 


$$
\frac{j_{01}}{\bar{j}}=\frac{2 k_{0}}{k_{\text {out }}} \cosh (e v / 2 k T) \text {. }
$$

Eq. 121 indicates that the kinetic limitation is more readily detectable at higher applied voltages.

The experimental results of steady state measurements are conveniently analyzed if presented in terms of normalized membrane conductance $G_{\infty}(V) / G_{\infty}(0)$, where $G_{\infty}(V)=e \bar{j} / V$ and $G_{\infty}(0)=\lim V \rightarrow C_{\infty}(V)$. As follows from the model, the normalized membrane conductance is equal to

$$
\frac{G_{\infty}(V)}{G_{\infty}(0)}=\left(\frac{2 k T}{e V}\right)\left(1+\frac{2 k_{0}}{k_{\text {out }}}\right) \frac{\sinh (e V / 2 k T)}{1+\frac{2 k_{0}}{k_{\text {out }}} \cosh (e V / 2 k T)} .
$$

The experimental results of transient membrane flux measurements are usualiy given in terms of the relaxation amplitude defined as

$$
\alpha=\frac{j(t=0)-\bar{j}}{\bar{j}} \text { and the relaxation time constant } \tau \text {. }
$$

Since $j(t=0)=j_{01}+\bar{j}($ see Eq. 116)

$$
\alpha=\frac{j_{01}}{\bar{j}}=\frac{2 k_{0}}{k_{\text {out }}} \cosh (\mathrm{eV} / 2 \mathrm{kT}) \text {, }
$$

and the relaxation time constant is

$\tau=\left[k_{\text {out }}\left\{1+\frac{2 k_{0}}{k_{\text {out }}} \cosh (e V / 2 k T)\right\}\right]^{-1}$.

It is therefore natural to define a kinetic limitation parameter, $E_{0}$, in the following way

$$
E_{0}=\frac{2 k_{0}}{k_{\text {out }}} \text {. }
$$

Now we describe the properties of the model in the limiting cases of slow and fast surface kinetics 
(a) Slow Interfacial Kinetics, $E_{0}>1$

In this approximation, the steady state flux is given by

$$
\vec{j}=k_{\text {in }} c_{C} \tanh (e V / 2 k T)
$$

One of the typical characteristics of steady state membrane flux limited by the kinetics of interfacial process is that it reaches saturation at higher applied voltages, and, second, its magnitude is limited by the rate of entry of species $C$ into the membrane.

The normalized membrane conductance becomes

$$
\frac{G_{\infty}(V)}{G_{\infty}(0)}=\frac{\tanh (e V / 2 k T)}{(e V / 2 k T)} .
$$

The relaxation amplitude and the relaxation time constant become

$$
\alpha=E_{0} \cosh (\mathrm{eV} / 2 \mathrm{kT})
$$

and

$$
\tau=2 \cdot k_{0} \cosh (e V / 2 k T)
$$

The relaxation amplitude and the time constant extrapolated to zero voltage yield directly the value of kinetic limitation parameter $E_{0}$ and the translocation rate constant $k_{0}$.

(b) Fast Interfacial Kinetics $E_{0}<1$

The steady state flux in this case is determined by the equilibrium density of membrane permeable species and their translocation rate constant. It is equal to

$$
\bar{j}=\left(k_{\text {in }} / k_{\text {out }}\right) c_{C} k_{0} \sinh (e V / 2 k T)
$$

Both the steady state flux and the normalized membrane conductance rapidly increase with the applied voltage due to the decrease of the 
height of membrane potential energy barrier.

$$
\frac{G_{\infty}(V)}{G_{\infty}(0)}=\frac{\sinh (e V / 2 k T)}{(e V / 2 k T)}
$$

As follows from Eq. 123 the relaxation amplitude is expected to be negligibly small, so that the transient component is not detectable.

In Figs. 21, 22, and 23 we have shown the voltage dependencies of the normalized membrane conductance, relaxation amplitude, and relaxation time constant. These quantities of experimental interest have been computed for various values of kinetic limitation parameter $E_{0}$.

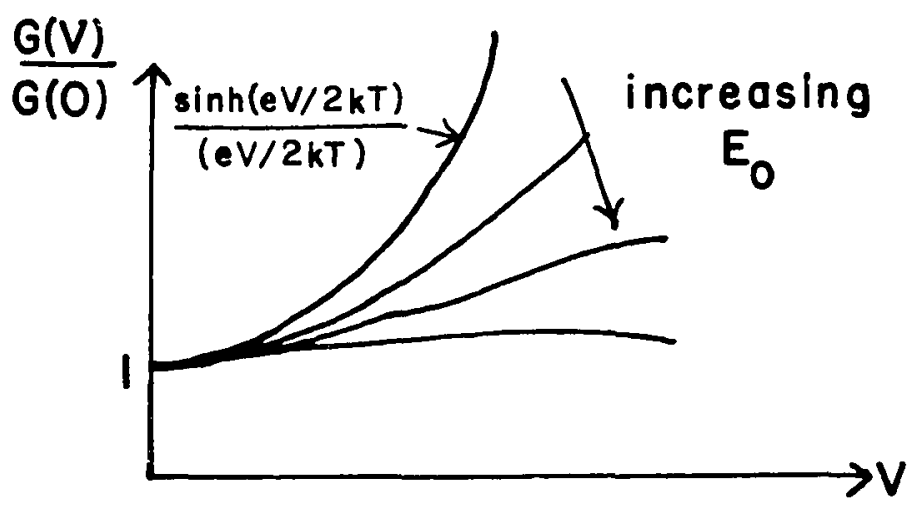

Figure 21. MODEL 4. Variation of the normalized membrane conductance with the voltage for various values of the kinetic limitation parameter $E$ (Eq. 132).

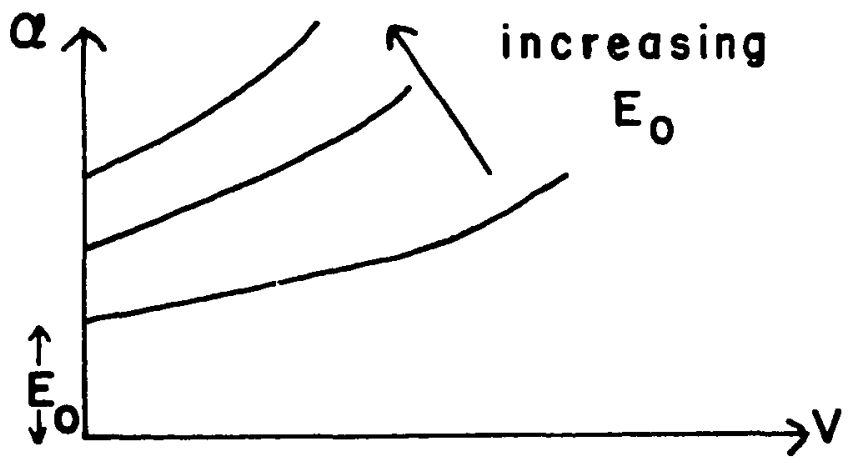

Figure 22. MODEL 4. Variation of the relaxation amplitude with the voltage for various values of the kinetic limitation parameter E (Eq. 123). 


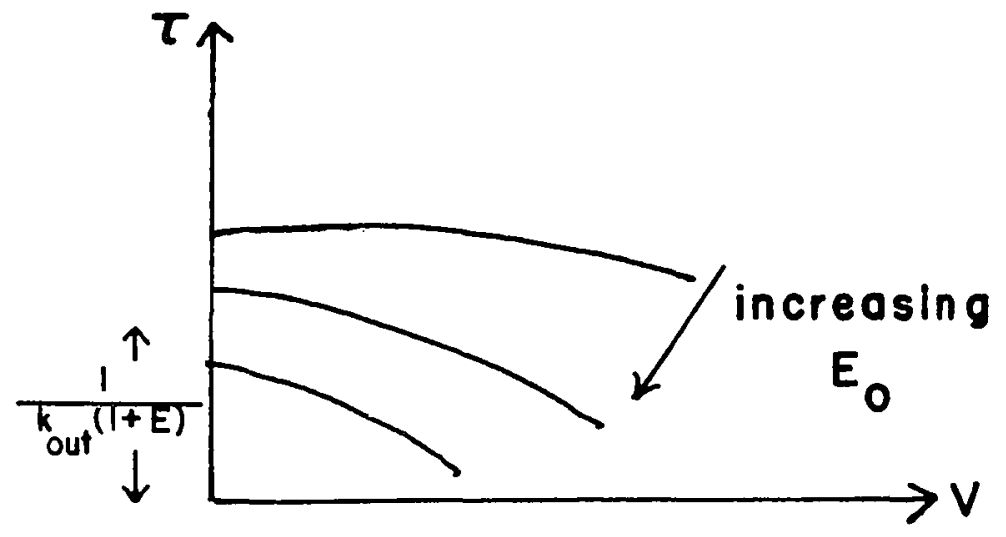

Figure 23. MODEL 4. Variation of the relaxation time constant with the voltage for various values of the kinetic limitation parameter E (Eq. 124).

The normalized steady state membrane conductance expressed in terms of the kinetic limitation parameter $E_{0}$ reads

$\frac{G_{\infty}(V)}{G_{\infty}(0)}=\frac{\sinh (e V / 2 k T)}{(e V / 2 k T)} \frac{1+E_{0}}{1+E_{0} \cosh (e V / 2 k T)}$

The same type of dependence of the normalized conductance on parameter $E_{0}$ has been predicted by more complicated models (see Eqs. 237 and 312). This observation has an important practical application. As the Fig. 21 shows, a deviation from the $\sinh (e V / 2 k T) /(e V / 2 k T)$ dependence of the normalized membrane conductance can be considered as an indication of existence of kinetic limitations.

MODEL 5: GENERAL SCHEME FOR SIMPLE CARRIER MECHANISMS

The model to be discussed in this section (Fig. 24) is a conceptual prototype of several membrane transport models. It has played an important role in the development of understanding of ion transport in lipid membranes because the model was found in reasonable agreement with the experimental results on carrier mediated ion transport driven by an applied electric field (52). 


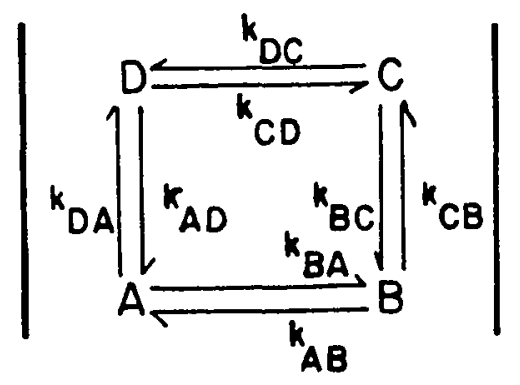

Figure 24. MODEL 5: General scheme for simple carrier mechanism.

Transformations D - A and B - C represent generalized reactions that can be associated with transfer of charge across the membrane water interfaces. Transformation A - B represents transfer of ions across the membrane, and the transformation $C$ - D can be considered to be a feedback process which completes the transport cycle. Due to the presence of the feedback process this scheme can support steady state flux. The model is described by the following set of equations:

$$
\begin{aligned}
& d n_{A} / d t=-\left(k_{B A}+k_{D A}\right) n_{A}+k_{A B} n_{B}+k_{A D} n_{D} \\
& d n_{B} / d t=k_{B A} n_{A}-\left(k_{A B}+k_{C B}\right) n_{B}+k_{B C} n_{C} \\
& d n_{C} / d t=k_{C B} n_{B}-\left(k_{B C}+k_{D C}\right) n_{C}+k_{C D} n_{D} \\
& d n_{D} / d t=k_{D A} n_{A}+k_{D C} n_{C}-\left(k_{A D}+k_{C D}\right) n_{D} \cdot
\end{aligned}
$$

As in many other cases of membrane transport models, the densities of components are not independent of each other because $\sum_{\alpha} d n_{\alpha} / d t=0$, so the above system of equations can be reduced to three by eliminating one of the variables using the conservation equation 


$$
n_{A}+n_{B}+n_{C}+n_{D}=N_{O}
$$

In this general case the solution for the net flux of ions consists of three transient and one steady state component.

Since most of membrane transport kinetic studies has been done on symmetric membranes, and also for the sake of greater mathematical simplicity, we will consider further only cases with symmetrical interfaces, i.e. $k_{B C}=k_{A D}, k_{C B}=k_{D A}$, and with the rate constants of the transformation $C-D$ equal to each other, i.e. $k_{C D}=k_{D C}=k_{S}$.

\section{System at Steady State}

The steady state densities $\bar{n}_{\alpha}$ are obtained by equating any three of the Eqs. 133 - 136 to zero and using Eq. 137. The steady state flux is given by

$$
\bar{j}=k_{B A} \cdot \bar{n}_{A}-k_{A B} \cdot \bar{n}_{B}
$$

and is equal to

$\bar{j}=\frac{N_{0}}{2} \frac{k_{B C}}{k_{C B}+k_{B C}} \frac{k_{B A}-k_{A B}}{1+\left(k_{B A}+k_{A B}\right) / k_{C B}+\left(k_{B C} / k_{C B}\right)\left(k_{B A}+k_{A B}\right) / 2 k_{S}}$.

if the charge transfer across the membrane core is the only field driven process, and the voltage dependence of $k_{B A}$ and $k_{A B}$ is given by Eqs. 120 , then the voltage dependence of the steady state flux is given by a rather simple expression

$$
\bar{j}(V)=N_{0} \frac{k_{B C}}{k_{C B}+k_{B C}} \frac{k_{0} \sinh (e V / 2 k T)}{1+E_{0} \cosh (e V / 2 k T)} .
$$

The quantity $E_{0}$ is equal to 


$$
E_{0}=2 k_{0} / k_{C B}+k_{B C} k_{0} / k_{C B} k_{S}
$$

and it can be used as a composite measure of kinetic limitations. When $E_{0} \ll 1, \bar{j}(V)$ becomes equal to the initial flux $j_{0}(V)$, and as the magnitude of the kinetic limitation parameter $E_{0}$ increases the steady state flux decreases. It will be shown later that displacement of densities of membrane permeable ions from their equilibrium values is proportional to $E_{0}$. It is convenient to express $E_{0}$ as a sum of two terms

$$
E_{0}=E_{1}+E_{2} \text {, }
$$

where $\quad E_{1}=2 k_{0} / k_{C B}$,

and $\quad E_{2}=k_{B C} k_{0} / k_{C B} k_{S}$.

$E_{1}$ is the kinetic limitation parameter associated with charge transfer process across the interface, and $E_{2}$ is pertinent to the characterization of the feedback pathway.

The normalized steady state membrane conductance $G_{\infty}(V) / G_{\infty}(0)$ is a suitable function for the evaluation of kinetic limitations from the steady state current-voltage data. It follows from Eq. 140 that

$$
G_{\infty}(V) / G_{\infty}(0)=\frac{\sinh (e V / 2 k T)}{(e V / 2 k T)} \frac{\left(1+E_{0}\right)}{1+E_{0} \cosh (e V / 2 k T)} .
$$

The effect of the magnitude of the kinetic limitation parameter $E_{0}$ on the voltage dependence of normalized membrane conductivity is illustrated in Fig. 25.

\section{Transient Process}

To determine the transient flux across the membrane we define the density displacements $x_{\alpha}$ in the usual manner 


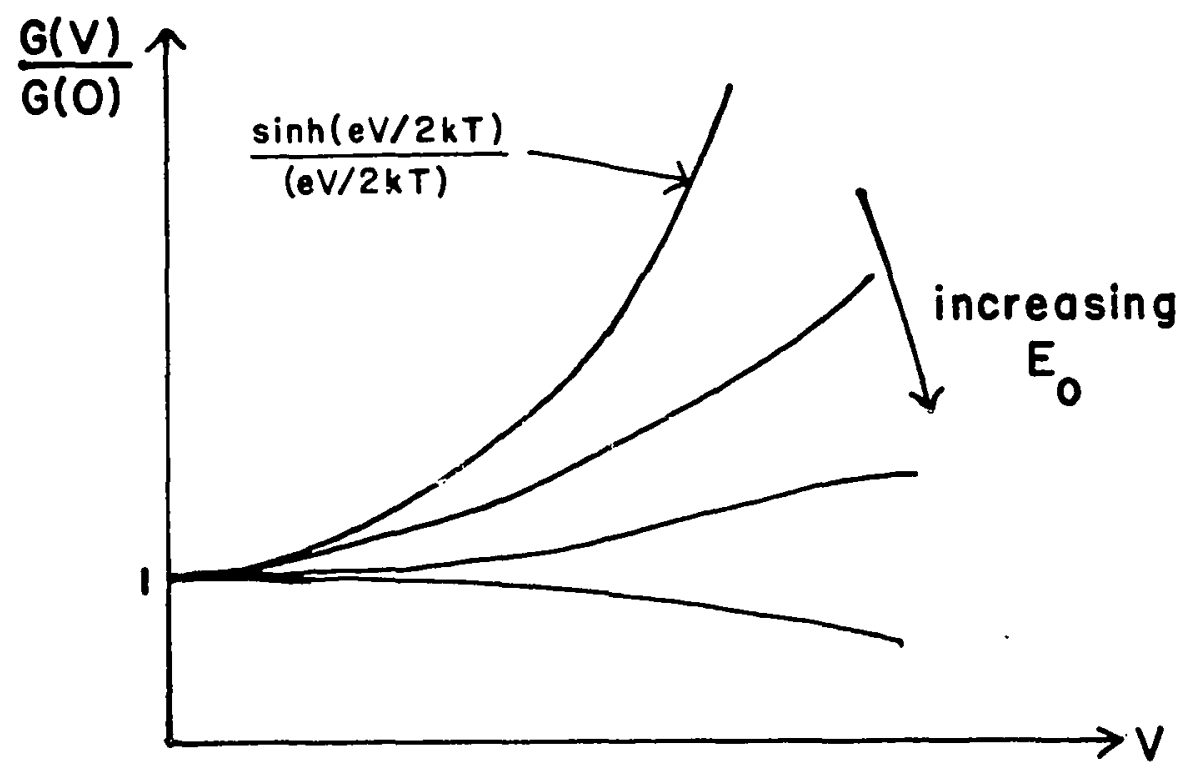

Figure 25. MODEL 5. Effect of the magnitude of the kinetic Iimitation parameter $E_{O}$ on the normalized membrane conductance curves (Eq. 145).

$$
\mathrm{n}_{\alpha}=\mathrm{n}_{\alpha}^{\mathrm{in}}+\mathrm{x}_{\alpha}, \quad(\alpha=A, B, C, D)
$$

where $n_{\alpha}^{i n}$ are the initial equilibrium densities. Because the present model is linear and symmetric, it can be shown that $x_{A}=-x_{B}$ and $x_{C}=-$ $\mathrm{x}_{\mathrm{D}}$ at all times. Using these properties it is possible to reduce the system of four differential Eqs. 133 - 136 to the following two,

$$
\begin{aligned}
& d x_{B} / d t=-\left(k_{B A}+k_{A B}+k_{C B}\right) x_{B}+k_{B C} x_{C}+n_{B}^{i n}\left(k_{B A}-k_{A B}\right), \\
& d x_{C} / d t=k_{C B} x_{B}-\left(k_{B C}+2 k_{S}\right) x_{C} .
\end{aligned}
$$

This system has a solution of the form

$$
x_{\alpha}={ }_{i=1}^{2} a_{\alpha i} \exp \left(-\lambda_{i} t\right)+\bar{x}_{\alpha} \quad(\alpha=B, C)
$$


where $\bar{x}_{\alpha}$ are the differences between the final steady state and the initial equilibrium densities, and $a_{\alpha i}$ are the density displacement amplitudes. The reciprocal relaxation time constants $\lambda_{i}$ are obtained from the characteristic equation

$\operatorname{det}\left(\begin{array}{cc}\lambda-\left(k_{B A}+k_{A B}+k_{C B}\right) & k_{B C} \\ k_{C B} & \lambda-\left(k_{B C}+2 k_{S}\right)\end{array}\right)=0$

The roots of the characteristic equation are

$$
\lambda_{1}=\tau_{1}^{-1}=a+b,
$$

and $\quad \lambda_{2}=\tau_{2}^{-1}=a-b$,

where

$$
a=\frac{1}{2}\left(k_{B A}+k_{A B}+k_{C B}+k_{B C}+2 k_{S}\right)
$$

and

$$
\mathrm{b}=\frac{1}{2}\left\{\left(\mathrm{k}_{\mathrm{BA}}+\mathrm{k}_{\mathrm{AB}}+\mathrm{k}_{\mathrm{CB}}-\mathrm{k}_{\mathrm{BC}}-2 \mathrm{k}_{\mathrm{S}}\right)^{2}+4 \mathrm{k}_{\mathrm{CB}} \mathrm{k}_{\mathrm{BC}}\right\}^{\frac{1}{2}}
$$

The ratio of the density displacement amplitudes is given by

$$
\frac{a_{C i}}{a_{B i}}=\frac{k_{B A}+k_{A B}+k_{C B}-\lambda_{i}}{k_{B C}}=L_{i} \text {. }
$$

$a_{B i}$ and $a_{C_{i}}$ are obtained from the initial equilibrium conditions,

$$
\begin{aligned}
& n_{B}^{\text {in }}=a_{B 1}+a_{B 2}+\bar{n}_{B}, \\
& n_{C}^{\text {in }}=L_{1} a_{B 1}+L_{2} a_{B 2}+\bar{n}_{C} .
\end{aligned}
$$

These are

$$
\begin{aligned}
& a_{B 1}=-\frac{L_{2}\left(\bar{n}_{B}-n_{B}^{i n}\right)-\left(\bar{n}_{C}-n_{C}^{i n}\right)}{L_{2}-L_{1}}=-a_{A 1}, \\
& a_{B 2}=-\frac{\left(\bar{n}_{C}-n_{C}^{i n}\right)-L_{1}\left(\ddot{n}_{B}-n_{B}^{i n}\right)}{L_{2}-L_{1}}=-a_{A 2} .
\end{aligned}
$$


The total flux is given by

$$
j(t)=k_{B A} \cdot n_{A}(t)-k_{A B} \cdot n_{B}(t)
$$

It is equal to

$$
\begin{aligned}
j(t) & =k_{B A} \stackrel{\Sigma}{i}=1_{A i} a_{A} \exp \left(-\lambda_{i} t\right)-k_{A B} \sum_{i=1}^{2} a_{B i} \exp \left(-\lambda_{i} t\right)+k_{B A} \bar{n}_{A}-k_{A B} \bar{n}_{B} \\
& =\left(k_{B A}+k_{A B}\right) \sum_{i=1}^{2} a_{A i} \exp \left(-\lambda_{i} t\right)+\bar{j} .
\end{aligned}
$$

In the experimental. studies the transient fluxes are usually measured with respect to the steady state flux,

$$
(j(t)-\bar{j}) / \bar{j}=r a_{1} \exp \left(-\lambda_{1} t\right)+r a_{2} \exp \left(-\lambda_{2} t\right),
$$

where $\mathrm{ra}_{1}$ and $\mathrm{ra}_{2}$ are the relaxation amplitudes of the fast and slow components. These are equal to

$$
r a_{i}=\left(k_{B A}+k_{A B}\right) a_{A i} / \bar{j}
$$

The relative contribution of the slow and fast component of the transient flux can be estimated from the ratio

$$
\frac{r a_{2}}{r a_{1}}=\frac{a_{A 2}}{a_{A 1}}=\frac{\left(\bar{n}_{C}-n_{C}^{i n}\right)-L_{1}\left(\bar{n}_{B}-n_{B}^{i n}\right)}{L_{2}\left(\bar{n}_{B}-n_{B}^{i n}\right)-\left(\bar{n}_{C}-n_{C}^{i n}\right)} .
$$

(a) Charge Translocation Across the Membrane as a Kinetically Slow Process

The transient component of the total ion flux is absent if the displacement amplitudes $a_{A i}=a_{B i}=0$, which occurs if the densities of species $A$ and $B$ are not displaced from their equilibrium values. We will show that the kinetic limitation parameter $E_{0}$, introduced earlier, is a measure of such displacement. The equilibrium density of species B is equal to 


$$
n_{B}^{\text {in }}=\left(N_{0} / 2\right) k_{B C} /\left(k_{B C}+k_{C B}\right)
$$

The density displacement of species B at steady state is

$\bar{x}_{B}=\bar{n}_{B}-n_{B}^{i n}=\frac{N_{0}}{2} \frac{k_{B C}}{k_{B C}+k_{C B}} \frac{\left(2 k_{S}+k_{B C}\right)\left(k_{B A}+k_{A B}\right)}{2 k_{S} k_{C B}+\left(2 k_{S}+k_{B C}\right)\left(k_{B A}+k_{A B}\right)}$.

The relative density displacement of species B from equilibrium is then given by

$$
\frac{\bar{n}_{B}-n_{B}^{i n}}{n_{B}^{i n}}=\frac{E_{0} \sinh (e V / 2 k T)}{1+E_{0} \cosh (e V / 2 k T)}
$$

which can also be shown to be equal to the relative density displacement of species A. Similarly, for species $C$ and $D$ we find

$$
\frac{\bar{n}_{C}-n_{C}^{i n}}{n_{C}^{i n}}=\frac{n_{D}^{i n}-\bar{n}_{D}}{n_{D}^{i n}}=\frac{E_{2} \sinh (e V / 2 k T)}{1+E_{0} \cosh (e V / 2 k T)} .
$$

Thus the transient flux is insignificant and the relative displacements of species are insignificant as well, when the kinetic limitation parameter $E_{0}$ is sufficiently small. Under these conditions the flux through the membrane is determined solely by the membrane potential energy barrier. The mutual relationship between displacements is depicted in Fig. 26. Due to the assumed symmetry of the transport system, the density displacements of pairs $A, B$, and $C, D$, are equal and opposite.

\section{(b) Fast Return Pathway}

The expression 168 shows that even in the presence of a significant kinetic limitation $\left(E_{0} \geq 1\right)$ it is possible to have the species $C$ and $D$ to remain close. to the initial equilibrium densities 


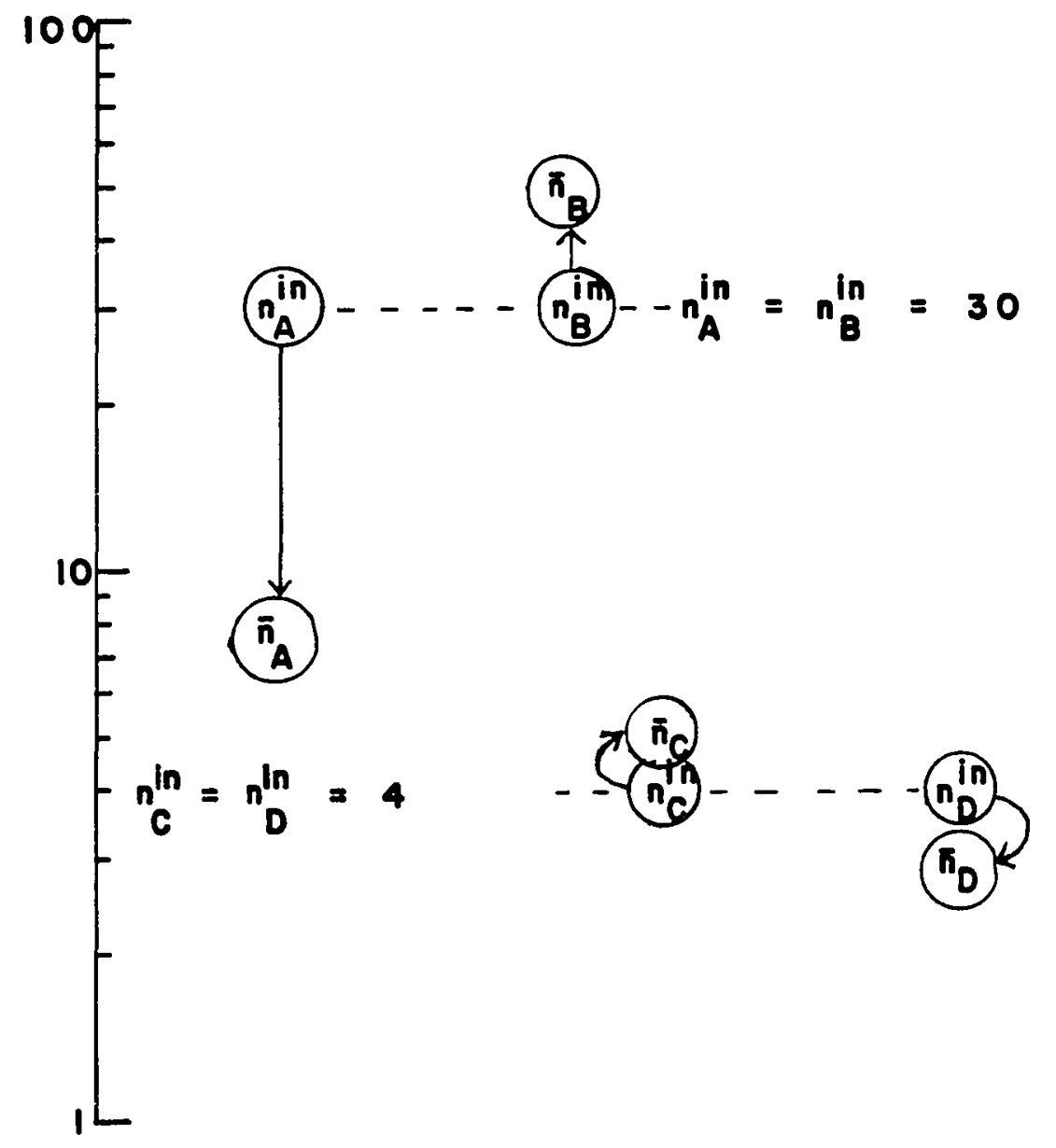

Figure 26. MODEL 5. Relationship between the relative displacements of the density of the species $A, B, C$, and $D$ from the initial equilibrium values $\left(n^{2 n}\right)$ to final steady state $(\bar{n})$ after the application of a potential difference across the membrane. Due to the assumed symmetry of the transport system, the density displacements of pairs $A, B$ and $C, D$ are equal and opposite. 
provided that $E_{2}<1$. This occurs when the rate of the return pathway $C-D$ is sufficiently fast. Furthermore, the densities of species $C$ and $D$ will be less displaced from the equilibrium if the equilibrium of the surface reaction is shifted towards $C$ rather than $B$.

We have seen that the general solution of the present model yields two components of transient flux. Approximate expressions for the relaxation time constants can be obtained by expanding the square root in Eqs. 151 and 152 into a power series. The reciprocal time constants of the fast and the slow processes become

$$
\lambda_{1}=\tau_{1}^{-1}=2 k_{S}+k_{B C},
$$

and $\lambda_{2}=\tau_{2}^{-1}=k_{C B}+k_{A B}+k_{B A}=k_{C B}\left(1+E_{1} \cosh (e V / 2 k T)\right)$.

The relaxation time constant of the fast component of the transient flux is primarily determined by the rate constant of the return pathway $\mathrm{k}_{\mathrm{S}}$ and is therefore independent of the voltage applied. The relaxation time constant of the slow component can also be obtained using the assumption that at sufficiently long times the density displacements $\mathrm{X}_{\mathrm{C}}$ and $\mathrm{X}_{\mathrm{D}}$ are zero. Then the system of Eqs. $147-148$ reduces to the following equation.

$$
d x_{B} / d t=-\left(k_{B A}+k_{A B}+k_{C B}\right) x_{B}+n_{B}^{i n}\left(k_{B A}-k_{A B}\right)
$$

Solution of this equation yields a relaxation time $\tau_{2}$ given by Eq. 170 . The voltage dependent part of the time constant provides information on the rate constant of the ion translocation across the membrane and the voltage independent part provides information on the rate constant of conversion of membrane permeable ions into components $C$ and $D$ whose 
translocation across the membrane is voltage independent.

The relative contribution of the fast and the slow component to the total flux can be estimated from the Eq. 164. Since $\left|L_{1}\right| \simeq \mid$ $-2 \mathrm{k}_{\mathrm{S}} / \mathrm{k}_{\mathrm{BC}} \mid \gg 1$ and $\mathrm{L}_{2} \approx 0$, the ratio of the relaxation amplitudes $r a_{2} / r a_{1}>1$, so that the slow component of the flux dominates.

(c) Fast Surface Reactions

We assume that $k_{S}$ and $k_{B A}+k_{A B}$ are small compared to $k_{C B}$ and

$k_{\mathrm{BC}}$ and that the rate constant of ion translocation is comparable to the rate constant of return pathway. In this case $E_{1} \ll 1$, so that $E_{0}=E_{2}$. Approximate expressions for the reciprocal relaxation times could be obtained as before by expanding the square root in Eq. 154 into a power series and neglecting the second and higher order terms. Then for the fast process we find that

$$
\begin{aligned}
\lambda_{1}= & \tau_{1}^{-1}=k_{C B}+k_{B C}+\left(k_{B A}+k_{A B}\right) /\left(1+k_{B C} / k_{C B}\right) \\
& +2 k_{S}\left(k_{B C} / k_{C B}\right) /\left(1+k_{B C} / k_{C B}\right),
\end{aligned}
$$

and for the slow process

$\lambda_{2}=\tau_{2}^{-1}=\left(k_{B A}+k_{A B}\right)\left(k_{B C} / k_{C B}\right)+2 k_{S} /\left(1+k_{B C} / k_{C B}\right)$

The time constant of the fast process is primarily determined by the kinetic constants of the surface reaction and it contains correction terms whose magnitude depends on whether the equilibrium at the surface is shifted towards the formation of membrane permeable ions or not. Eq. 173 indicates how the kinetics of the surface reaction influence the time constant of the slow component of ion flux. If the equilibrium of the surface reaction is shifted towards the formation of membrane 
permeable ions, the relative contribution of the ion translocation kinetics is greater. Voltage dependence of the slow relaxation time constant should indicate the influence of the equilibrium constant of the surface reaction. The time constant of the slow process could also be obtained froln the fact that at sufficiently long time after the application of a voltage step the surface reaction is close to equilibrium, so that the time evolution of the membrane transport system corresponds to

$$
\frac{d}{d t}\left(x_{B} / n_{B}^{i n}\right)=\frac{d}{d t}\left(x_{C} / n_{C}^{i n}\right)
$$

This equation states that the rates of change of relative displacement of species $B$ and $C$ from the equilibrium are the same. We find again that $\mathrm{ra}_{2} / \mathrm{ra}_{1} \gg 1$ so that the slow process dominates the transient flux.

Figs. 27 - 29 illustrate the voltage dependence of the relaxation time constants and the relaxation amplitudes of membrane ion flux obtained from a general solution of the model without approximations. Fig. 27 shows the case for fast return pathway when the displacement of species $A$ and $B$ is due to the limiting rate of the transformation $B$ C. Fig. 28 corresponds to the case of fast surface process where the decrease in transmembrane ion flux is due to the slow return pathway. In both cases the time constant of the fast transient is essentially voltage independent because the fast relaxation process is due to the redistribution of densities of components along the voltage independent routes. The relaxation process is dominated by the slow component whose time constant is strongly voltage dependent. These figures also point to the difficulties of analysis of experimental results when the fast relaxation process cannot be either resolved or measured with suffi- 

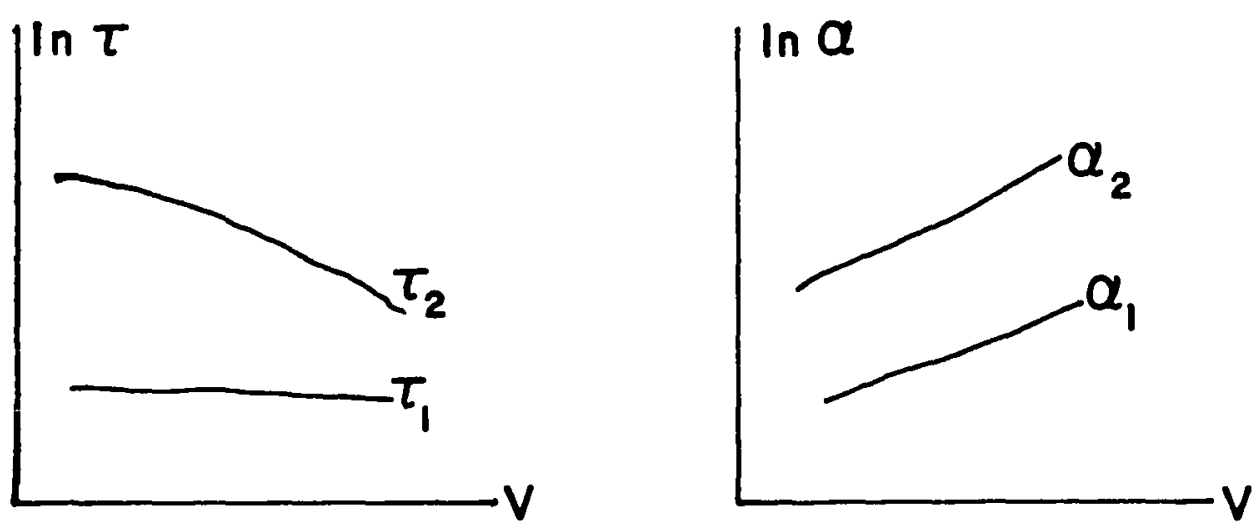

Figure 27. MODEL 5. SLOW SURFACE REACTION. Variation of the relaxation characteristics with the voltage for model 5 when the surface reaction is slow and the rate of backflow is fast $\left(k_{0}=1, k_{S}=165, k_{B C}=66, k_{C B}=20\right)$.
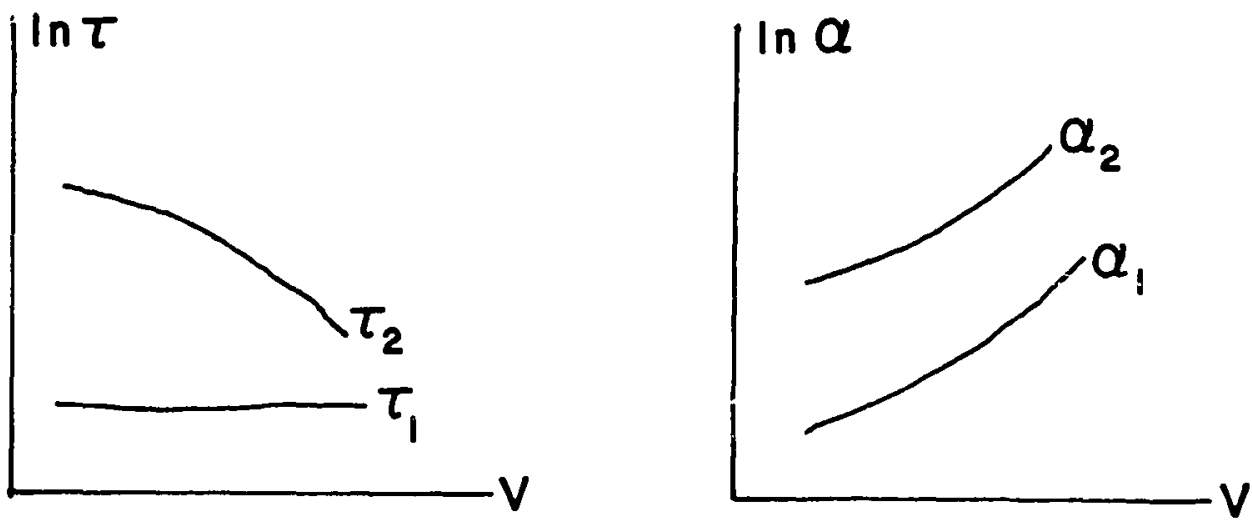

Figure 28. MODEL 5. FAST SURFACE REACTION. Variation of the relaxation characteristics with the voltage for model 5 when the surface reaction is fast and the rate of backflow is slow $\left(k_{0}=1, k_{S}=33.3, k_{B C}=167, k_{C B}=50\right)$.

cient accuracy. In this case one cannot determine whether the membrane transport is kinetically limited by the slow surface kinetics or by the return pathway. It is therefore necessary to have experimental control over the rate constants of surface process and of return pathway. Such a control can be accomplished in some cases by changing the composition of the aqueous medium, varying the structure of the membrane water interface region, and varying the membrane thickness (55-57).

Under favorable conditions it is possible to observe transi- 
tions from one kinetic regime into another. Such a transition is related to the interchange of dominating relaxation amplitudes. One such example is shown in Fig. 29. At low voltages the transient flux is
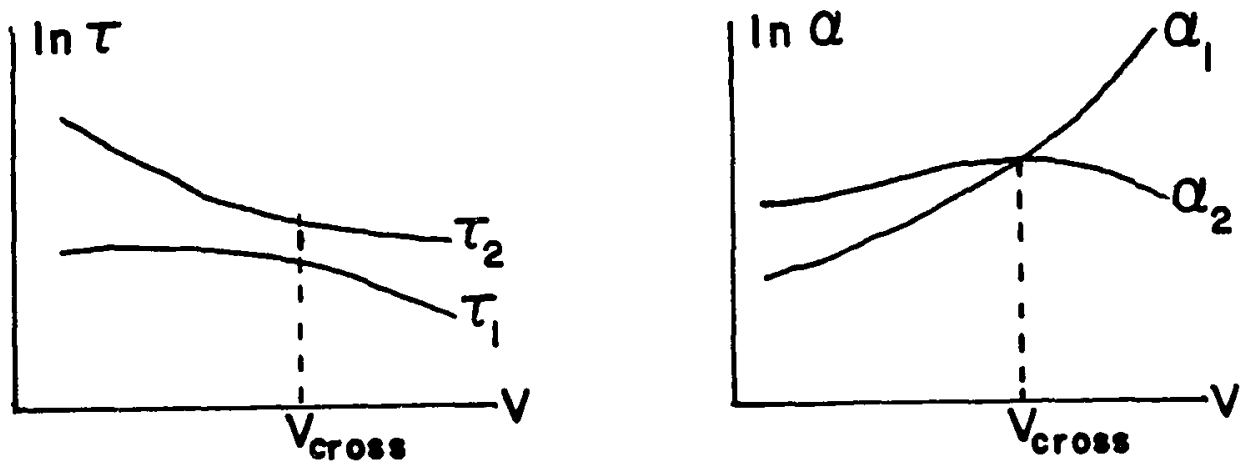

Figure 29. MODEL 5. Illustration of the transition from one kinetic regime to another with the applied voltage. In this example the rate constants were set as follows: $k_{0}=1, k_{s}=165$, $\mathrm{k}_{\mathrm{BC}}=20.2, \mathrm{k}_{\mathrm{CB}}=6.06$. Below $\mathrm{v}$ cross the relaxation amplitude is dominated by the slow process, whereas at $V>V$ cross it is dominated by the fast process.

dominated by the slow component, whereas at high voltage the fast component dominates. The interchange occurs when $\mathrm{ra}_{2}=\mathrm{ra} \mathrm{a}_{1}$ and as follows from Eq. 164, it corresponds to

$$
\left(\bar{n}_{C}-n_{C}^{i n}\right) /\left(\bar{n}_{B}-n_{B}^{i n}\right)=\left(L_{1}+L_{2}\right) / 2
$$

In the case chosen for illustration the rate constants were such that $E_{1}>E_{2}$ and $k_{S}>\left(k_{C B}, k_{B C}\right)>k_{0}$. In this specific case the dominating limitation is due to the slow kinetics of transformation B - C. Thus, at low voltages the fast component of the transient flux is mainly due to the redistribution of densities of species $C$ and $D$ along the return pathway. The slow component of transient flux, which is the dominating one, corresponds to redistribution along the translocation and surface transformation pathway. Above the transition point the rate constant of ion translocation in forward direction $k_{B A}$ becomes the largest one due 
to the applied voltage, and the ion translocation in forward direction becomes the fastest process. Also, above the transition voltage the fast component of the transient flux dominates. The voltage range within which such interchange takes place depends on the ratio $\mathrm{k}_{\mathrm{CB}} / \mathrm{k}_{\mathrm{O}}$ ' $\mathrm{k}_{\mathrm{BC}} / \mathrm{k}_{\mathrm{CB}}$ and $\mathrm{k}_{\mathrm{S}} / \mathrm{k}_{0}$. If $\mathrm{k}_{\mathrm{S}}>\mathrm{k}_{\mathrm{O}}>\left(\mathrm{k}_{\mathrm{BC}}, \mathrm{k}_{\mathrm{CB}}\right)$ the transition is sharper. Similar change of the kinetic regime (not shown) occurs for $E_{1}<E_{2}$. In this particular case the transformations A - D and B - C are relatively fast ones, so that at low voltage the surface reactions rapidly reach near equilibrium state, and the dominating limitation is associated with the redistribution along the slow ion translocation and return pathways. At high voltages, above the transition point, the ion translocation in the forward direction becomes the fastest process and the transient flux also becomes dominated by the fast component.

\section{MODEL 6: CLASS I UNCOUPLERS}

One of the applications of the general scheme of carrier mechanism is to the charge transport in lipid membranes induced by uncouplers of oxidative phosphorylation. For uncouplers of class I the charge flux in membrane is proportional to the concentration of uncoupler molecules and it can be therefore assumed that the kinetic scheme consists of a sequence of linear elementary processes. If, in addition, the uncoupler partitions very strongly into the membrane, it can be assumed that over a short time interval the adsorption and desorption rates are limited by the diffusion in the aqueous medium and therefore are rather small. Hence the distribution of uncoupler is primarily determined by the kinetics of intramembrane transport initiated by the applied voltage, and not by the above mentioned adsorption-desorption processes. 
The kinetic scheme to be discussed is presented in Fig. 30. The charged membrane permeable species is the anion $A^{-}$, which also acts as a carrier of hydrogen ions in the membrane. This feature is considered to be essential for the ability of these molecules to dissipate the differences of concentration of hydrogen ions and of electric potential across energy transducing membranes. Due to the externally applied electric field the anions $\mathrm{A}^{-}$are driven across the membrane by electrodiffusion, which in the presence of a kinetic limitation results in the buildup of anions $A^{-}$at the positively biased side of the membrane and simultaneously in the depletion of $\mathrm{A}^{-}$at the negative side.

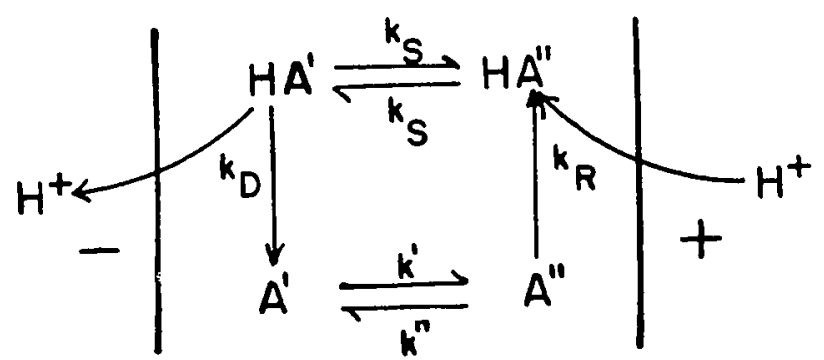

Figure 30. MODEL 6. Kinetic scheme for class I uncouplers.

In view of the general kinetic scheme of carrier transport the new kinetic constants are defined in the following way,

$$
\mathrm{k}_{\mathrm{CB}}=\mathrm{k}_{\mathrm{DA}}=\mathrm{k}_{\mathrm{R}} \mathrm{a}_{\mathrm{H}} \text {, }
$$

where $a_{H}$ is the proton activity and

$$
\mathrm{k}_{\mathrm{BC}}=\mathrm{k}_{\mathrm{AD}}=\mathrm{k}_{\mathrm{D}}
$$


The reduced hydrogen ion concentration is

$$
\mathrm{h}=\mathrm{a}_{\mathrm{H}} / \mathrm{K}_{\mathrm{a}}=10^{\mathrm{pK}} \mathrm{a}^{-\mathrm{pH}} \text {, }
$$

where $\mathrm{K}_{\mathrm{a}}$ is the dissociation constant of the uncoupler given by

$$
K_{a}=k_{D} / k_{R}
$$

Properties of Steady state Flux

At steady state flux of ions $A^{-}$across the membrane is also equal to the backward flux of neutral molecules HA. It is given by

$\bar{j}(v)=\frac{N_{0}}{1+h} \frac{k_{0} \sinh (e V / 2 k T)}{\left\{1+2 k_{0} / k_{R} a_{H}+k_{D} k_{0} / k_{R} k_{S} a_{H}\right\} \cosh (e V / 2 k T)}$.

It follows that the kinetic limitation parameter $E_{0}$ consists of two terms

$$
\begin{aligned}
E_{0} & =h^{-1}\left(2 k_{0} / k_{D}+k_{0} / k_{S}\right) \\
& =E_{1}+E_{2} .
\end{aligned}
$$

The kinetic parameter $E_{1}$ given by

$$
E_{1}=2 k_{0} / k_{R} a_{H}
$$

is associated with the kinetics of interfacial hydrogen ion transfer. $E_{1}$ becomes large at high $\mathrm{pH}$ indicating that the buildup and depletion of $A^{-}$at the opposite sides of the membrane are due to the slow rate of interfacial hydrogen ion transfer. The kinetic limitation parameter $\mathrm{E}_{2}$ given by

$$
E_{2}=k_{D} k_{0} /\left(k_{R} a_{H} k_{S}\right)
$$


is a measure of relative displacement of the density of neutral molecules $\mathrm{HA}$ from the initial equilibrium. The magnitude of $\mathrm{E}_{2}$ increases with the $\mathrm{pH}$. At low hydrogen ion concentration in the aqueous medium the density of $\mathrm{HA}$ is small compared with that of $\mathrm{A}^{-}$so that the ability of return pathway to support a high initial flux becomes 1 imited.

The zero voltage conductivity $G(0)$, which is obtained as a limit of $G(V)$ as $V$ tends to zero, is equal to

$$
G(0)=\frac{e^{2} N_{0} k_{0}}{2 k T} \frac{1}{(1+h)\left(1+E_{0}\right)}
$$

The conductivity is $\mathrm{pH}$ dependent and has a maximum value at $\mathrm{pH}=\mathrm{pH}_{\text {gmax }}$ given by

$$
\mathrm{pH}_{\text {gmax }}=\mathrm{pK} a-\frac{1}{2} \log _{10}\left(2 k_{0} / k_{D}+k_{0} / k_{S}\right)
$$

This equation indicates that the position of conductivity maximum on the $\mathrm{pH}$ scale depends on the magnitude of rate constants for the interfacial and return pathways. Thus, when the kinetics of these pathways is fast compared to the transmembrane charge translocation, i.e. $k_{0}\left(2 / k_{D}+1 / k_{S}\right)<1$, the conductivity maximum occurs at a pH exceeding $\mathrm{pK}_{\mathrm{a}}$. On the other hand, when the charge transport across the membrane becomes limited either by the interface or return pathway kinetics, the conductivity maximum occurs at a $\mathrm{pH}$ smaller than $\mathrm{pK}_{\mathrm{a}}$. In the absence of knowledge of $\mathrm{N}_{0}$ the kinetic limitation parameter $E_{0}$ can be determined from the voltage dependence of the normalized membrane conductivity $G(V) / G(0)$ given by

$$
\frac{G(V)}{G(0)}=\frac{\sinh (e V / 2 k T)}{(e V / 2 k T)} \quad \frac{1+E_{0}}{1+E_{0} \cosh (e V / 2 k T)}
$$


Transient Flux Parameters

Since it is not possible to distinguish between the two types of kinetic limitations neither from the voltage dependence of normalized membrane conductance, nor from the $\mathrm{pH}$ value of conductivity maximum, we explore now the possibility to use the voltage and $\mathrm{pH}$ dependencies of relaxation time constants and relaxation amplitudes for the purpose of determination of the slowest process. It follows from the above description that the total relaxation amplitude will increase with the $\mathrm{pH}$ for the simple reason that the kinetic limitations increase with the $\mathrm{pH}$. The relaxation time constants are given by Eqs. 151-154 after substituting the new interfacial rate constants for $k_{B C}$ and $k_{C B}$.

Fast Return Pathway

If the rate constant of translocation of neutral molecules HA is greater than the rate constant of interfacial release of hydrogen ions ( $i . e ., k_{S}>k_{D}$ ), then $E_{1}>E_{2}$. When the system is displaced from equilibrium by the applied voltage, the current decays with time due to (a) the depletion of $A^{-}$on the negatively biased membrane side, caused by slow rate of dissociation of $\mathrm{HA}$, and (b) due to accumulation of $\mathrm{A}^{-}$ at the positively biased surface, caused by slow rate of association of $\mathrm{A}^{-}$with hycirogen ion. At high $\mathrm{pH}$ the density of $\mathrm{HA}$ is small compared to that of $A^{-}$so that at the negative side of the membrane the rate of release of hydrogen ions cannot keep up with the rate of translocation of $\mathrm{A}^{-}$towards the positively biased side. Also on the positively biased surface the rate of influx of $\mathrm{A}^{-}$is greater than the rate of conversion into the neutral form due to low concentration of hydrogen ions. Thus, at high $\mathrm{pH}$ the flux is further reduced from the initial flux. The time 
constant of the fast process is given by

$$
\tau_{1} \simeq\left(2 \mathrm{k}_{\mathrm{S}}\right)^{-1}
$$

Since this relaxation time constant is mainly determined by the rate constant of the return pathway, it is both $\mathrm{pH}$ and voltage independent. The computer analysis of the model showed that the relaxation amplitude of the fast process is small unless the $A^{-}$translocation rate constant becomes (due to the applied voltage) comparable with $\mathrm{k}_{\mathrm{S}}$, and unless $\mathrm{pH}^{<} \mathrm{pK}_{\mathrm{a}} \cdot$

The time constant of the slow component is given by

$\tau_{2} \simeq\left[2 k_{0} \cosh (e V / 2 k T)+k_{D} h\right]^{-1}=\left[k_{D} h\left\{E_{1} \cosh (e V / 2 k T)+1\right\}\right]^{-1}$.

At high $\mathrm{pH}$ the time constant $\tau_{2}$ is more voltage dependent than at low $\mathrm{pH}$. It is to be expected that at high $\mathrm{pH}$ the relaxation characteristics of uncouplers of class I should resemble those of membrane permeable anion tetraphenylborate. Several of the characteristic features of the model for fast return kinetics are illustrated in Fig. 31 which shows the voltage and $\mathrm{pH}$ dependencies of the relaxation time constants and amplitudes computed from the general model without approximations.

\section{Fast Interface Kinetics}

In this case $k_{D}>k_{S}$, so that $E_{2}>E_{1}$ and the main relaxation process is associated with the displacement of densities of neutral molecules from their equilibrium values, which in turn also results in the accumulation of $\mathrm{A}^{-}$on the positive membrane surface and in the depletion of $\mathrm{A}^{-}$on the negative surface. The time constant of fast 

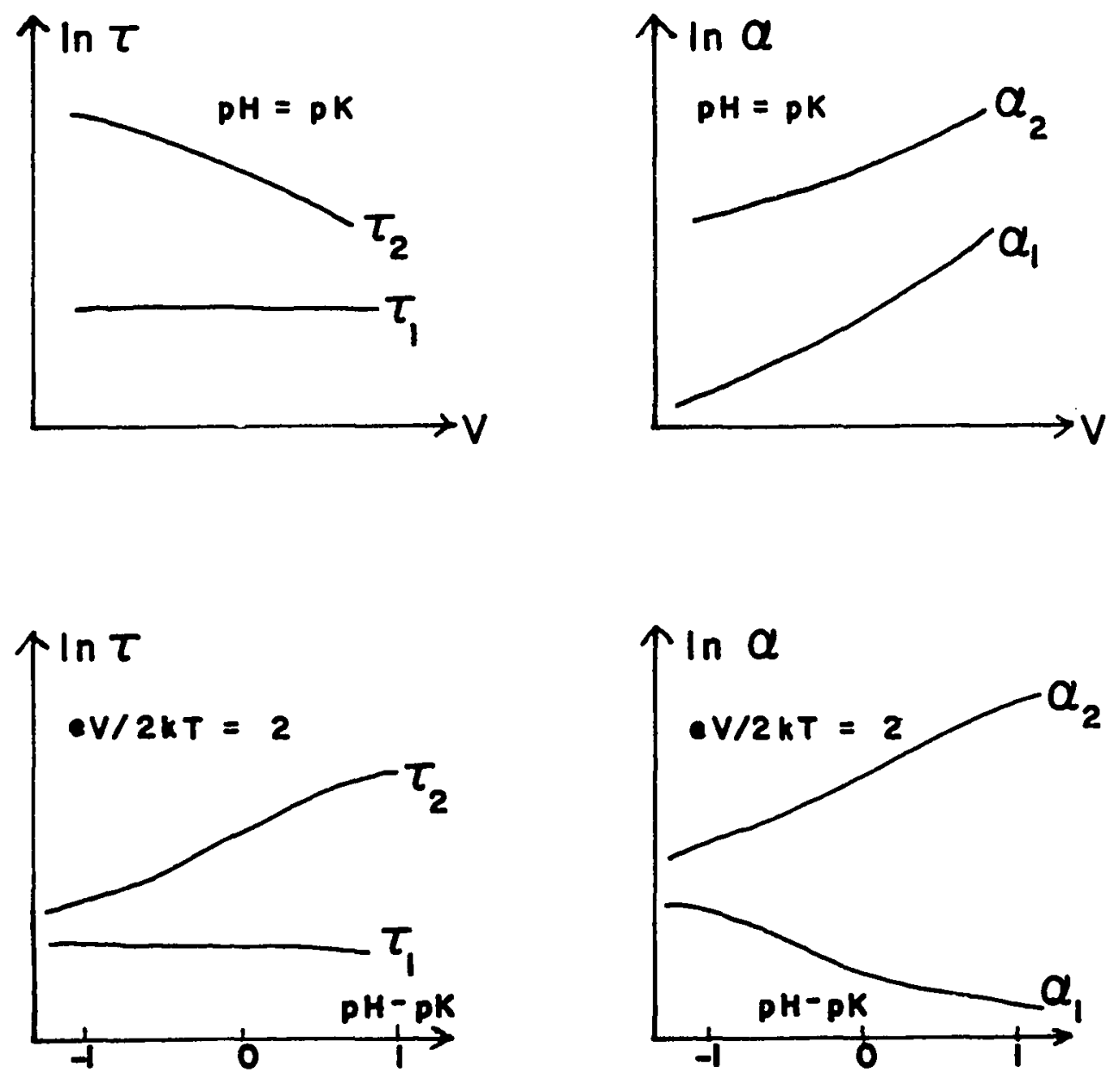

Figure 31. MODEL 6. FAST RETURN FLOW. Variation of the time constant and the relaxation amplitude with the voltage $(\mathrm{pH}=\mathrm{pK})$ and the $\mathrm{pH}(\mathrm{eV} / 2 \mathrm{kT}=2)$, for model 6 when the rate of return flow of $\mathrm{HA}$ is fast. The fast time constant $T$ is independent of the voltage and the $\mathrm{pH}(\mathrm{Eq} \cdot 187)$. The relaxation amplitude of the slow transient process increases with the $\mathrm{pH}$. 
relaxation component is approximately equal to

$$
\tau_{1} \simeq\left[k_{D}(1+h)+\frac{h}{1+h}\left(1+2 k_{0} \cosh (e v / 2 k T)+\frac{2 k_{S}}{h}\right]^{-1}\right.
$$

whose value is dominated by the kinetic constants of interfacial hydrogen ion transfer. The terms containing the ion translocation rate constant, $\mathrm{k}_{0}$, and that of return pathway, $\mathrm{k}_{\mathrm{S}}$, are of secondary importance. The main feature of the fast relaxation time constant is that it increases with the increase of $\mathrm{pH}$.

For slow relaxation component we obtain

$$
\tau_{2} \simeq\left[\frac{2 k_{0}}{h} \cosh (e V / 2 k T)+\frac{2 k_{s} h}{1+h}\right]^{-1} \text {, }
$$

which indicates again that at high $\mathrm{pH}$ the relaxation time constant of slow component becomes strongly voltage dependent. Under this condition its value reflects the time constant of ion translocation across the membrane, a situation similar to that observed for negatively charged lipophilic ions (Eq. 69). At low pH the time constant reflects the contribution of return pathway kinetics.

For comparison we have shown in Fig. 32 the voltage and the $\mathrm{pH}$ dependencies of relaxation parameters computed from the general model for the same set of rate constants as in Fig. 31, except that the values of $k_{S}$ and $k_{D}$ have been interchanged. These results illustrate the effect of fast surface kinetics and the features discussed above.

\section{CLASS II UNCOUPLERS: INTRODUCTION}

Characteristic feature of action of class II uncouplers in lipid membranes is that the induced membrane conductance increases with the square of the uncoupler concentration. The experimental results 

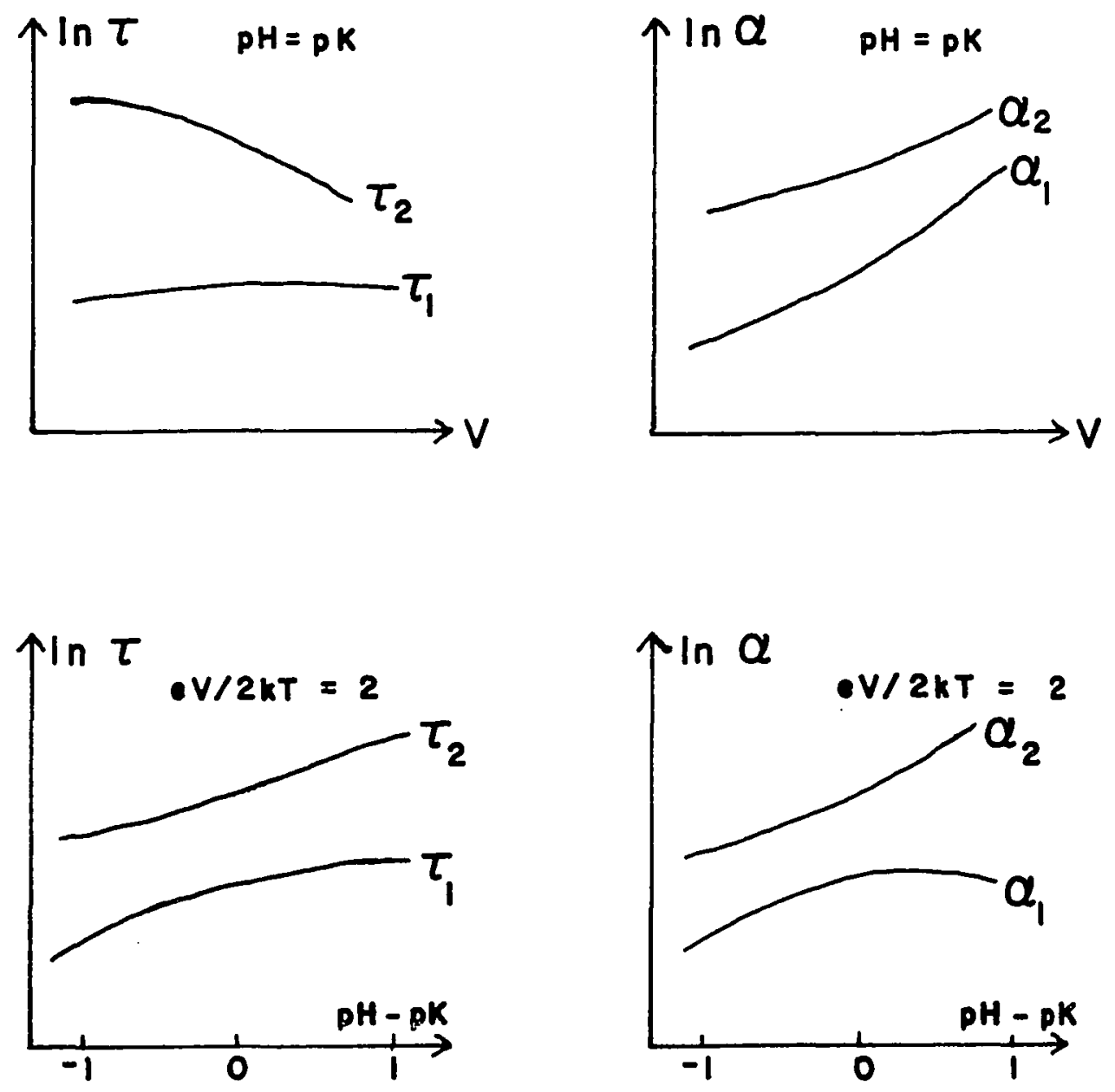

Figure 32. MODEL 6. FAST INTERFACIAL REACTION. Variation of the time constant and the relaxation amplitude with the voltage $(\mathrm{pH}=\mathrm{pK})$ and the $\mathrm{pH}(\mathrm{eV} / 2 \mathrm{kT}=2)$, for model 6 when the rate of interfacial reaction is fast. 
were found to be consistent with the byprthesis that charged membrane permeable species is a dimer complex, which is a product of combination of one neutral and one ionized uncoupler molecule. In earlier studies $(8,28,30)$ it was shown that dimer complexes cannot be supplied by any formation process taking place in the bulk aqueous medium, because the measured membrane conductance is far greater than that associated with the diffusion of ions in aqueous medium. There is a good experimental evidence indicating that the charge flux in the aqueous medium is carried by hydrogen ions and the electrolyte (28).

Our objective in this section is to consider two kinetic models that appear to be relevant to our experimental results. The models differ by the nature of charge transfer across the membrane water interface. In the first model the charge flow is maintained by hydrogen ions; in the second model, by uncoupler anions. Justification for these rather detailed theoretical studies is that the first model was found to be consistent with the results of earlier steady state studies on pentachlorophenol induced membrane conductance (28). The second model represents an attempt to interpret experimental results obtained with pentachlorobenzenethiol, a toxic substance whose molecular structure differs from that of pentachlorophenol only by replacement of oxygen by sulfur.

MODEL 7: DIMER MODEL A

Description of the Model

The kinetic scheme of membrane transport is shown in Fig. 33 . The uncoupler acts like a weak acid and so is represented by HA. The charge is assumed to be carried across the membrane by the dimer $\mathrm{HA}_{2}^{-}$ 


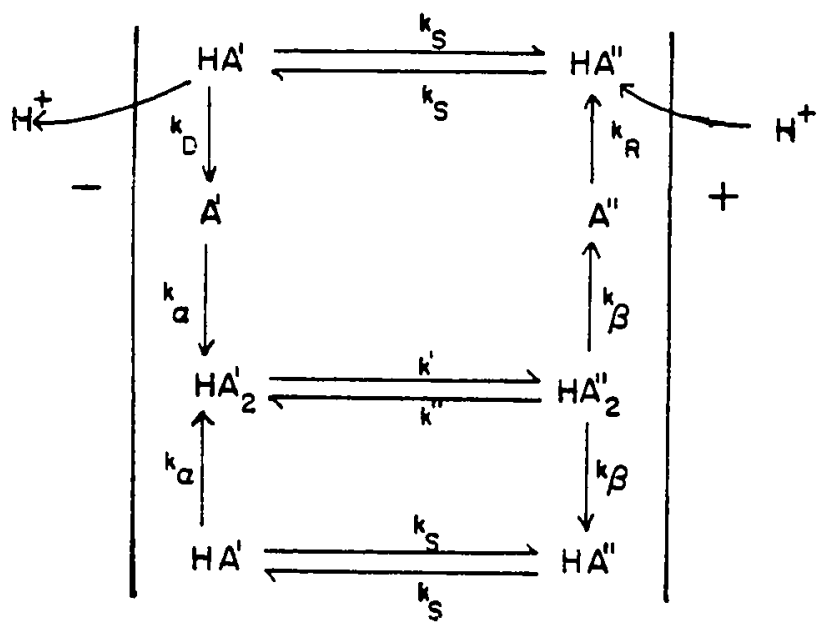

102

Figure 33. MODEL 7. Transport scheme for the dimer model A.

formed by the association of $\mathrm{HA}$ and $\mathrm{A}^{-}$species adsorbed at the membrane surface. The charge is assumed to be carried across the membrane water interface by the protons. The neutral HA molecules but not the anions $\mathrm{A}^{-}$are assumed to be membrane permeable.

Consider that the right hand side of membrane is positively biased. Then the net movement of all components constituting the kinetic scheme is as follows. Dimers are formed at the left hand surface by the combination of $\mathrm{HA}$ and $\mathrm{A}^{-}$species and diffuse across the membrane by the externally applied electric field. On the right hand side of the membrane the dimers dissociate to produce molecules of $\mathrm{HA}$ and $\mathrm{A}^{-}$ions. The excess $\mathrm{HA}$ molecules formed tend to diffuse back to the left hand side of the membrane. Excess $\mathrm{A}^{-}$anions on the right hand side combine with protons from the right aqueous phase to form HA molecules which again contribute to back diffusion to the left hand side of the membrane. The HA molecules that arrive at the left hand surface dissociate with the release of protons to the left aqueous phase and form $A^{-}$anions. These anions combine with neutral HA molecules to form dimers $\mathrm{HA}_{2}^{-}$and complete the cycle. During one cycle a proton is transported across the membrane. 
The model is characterized by the following set of six differential equations:

$$
\begin{aligned}
& d n_{A^{\prime}} / d t=k_{D^{\prime} A^{\prime}}-k_{R} a_{H^{\prime} A^{\prime}}+k_{B^{\prime}} n_{H A}{ }_{2}-k_{\alpha} n_{A^{\prime}} n_{H A^{\prime}}, \\
& d n_{A}{ }^{\prime \prime} / d t=k_{D} n_{H A}=-k_{R} a_{H} n^{\prime \prime}+k_{B} n_{H A}{ }_{2}-k_{\alpha} n_{A^{\prime}} n_{H A} \text {, } \\
& d n_{H A}{ }^{\prime} / d t=k_{R_{H}} a_{H^{\prime}} n^{\prime}-k_{D_{H A}} n_{H^{\prime}}+k_{B} n_{H A}{ }_{2}-k_{\alpha_{A}} n_{A^{\prime}} n_{H A^{\prime}}+j_{H A} \text {, } \\
& d n_{H A^{\prime \prime}} / d t=k_{R} a_{H^{\prime}} n^{\prime}-k_{D} n_{H A}{ }^{\prime}+k_{B} n_{H A}{ }_{2}-k_{\alpha A^{\prime \prime}} n_{H A}{ }^{\prime \prime}-j_{H A} \text {, } \\
& d n_{H_{2}^{\prime}} / d t=k_{\alpha} n_{A^{\prime}} n_{H A^{\prime}}-k_{\beta} n_{H A_{2}^{\prime}}{ }^{-j}, \\
& d n_{\mathrm{HA}_{2}^{\prime \prime}} / d t=k_{\alpha A^{\prime \prime}} n_{H A^{\prime \prime}}-k_{B} n_{H A_{2}^{\prime \prime}}+j \text {, }
\end{aligned}
$$

where the charge flux $j$ and the return flux of neutral molecules $j_{\mathrm{HA}}$ are given by

$$
j=k^{\prime} n_{H_{2}^{\prime}}-k^{\prime \prime} n_{H A_{2}^{\prime \prime}}
$$

and $j_{\mathrm{HA}}=\mathrm{k}_{\mathrm{S}}\left(\mathrm{n}_{\mathrm{HA}}{ }^{\prime \prime}-\mathrm{n}_{\mathrm{HA}}{ }^{\prime}\right)$.

From the inspection of the above system of equations it follows that

$\frac{d}{d t}\left(n_{A^{\prime}}+n_{A^{\prime \prime}}+n_{H^{\prime}}+n_{H^{\prime \prime}}+2 n_{H_{2}^{\prime}}+2 n_{H A_{2}^{\prime \prime}}\right)=0$

indicating that the densities of species $A^{\prime}, H A^{\prime}, \mathrm{HA}_{2}^{\prime}, \mathrm{A}^{\prime \prime}, \mathrm{HA}^{\prime \prime}$, and $\mathrm{HA}_{2}^{\prime \prime}$ are not independent of each other, so that one of the differential equations can be replaced by the conservation equation

$$
n_{A^{\prime}}+n_{A^{\prime \prime}}+n_{H A^{\prime}}+n_{H^{\prime \prime}}+2 n_{H A_{2}^{\prime}}+2 n_{H A_{2}^{\prime \prime}}=N_{0}
$$

where $\mathrm{N}_{0}$ is the total density of uncoupler in all forms on both sides of the membrane. 
The objective of solving the above system of equations is to determine the ion flux across the membrane $j$ as a function of total density of uncoupler adsorbed at the membrane $N_{0}$, hydrogen ion activity $a_{H}$, and electric potential difference $V$ applied across the membrane.

\section{Steady State Properties}

The steady state distribution of densities of species $A^{-}, H A$, and $\mathrm{HA}_{2}^{-}$are obtained by setting the time derivatives in Eqs. 191-196 to zero. The system of algebraic equations thus obtained can be further reduced to the following system:

$$
\begin{aligned}
& \bar{j}_{H A} / 2=k_{\beta} \bar{n}_{H A}{ }_{2}-k_{\alpha} \bar{n}_{H A^{\prime \prime}} \bar{n}_{A^{\prime \prime}}, \\
& \bar{j}_{H A} / 2=k_{\alpha} \bar{n}_{H A} \bar{n}_{A^{\prime}}-k_{B^{\prime}} \bar{n}_{H A}, \\
& \bar{j}_{H A} / 2=k_{R^{\prime}} \bar{n}_{A^{\prime \prime}} a_{H}-k_{D} \bar{n}_{H A^{\prime \prime}}, \\
& \bar{j}_{H A} / 2=k_{D} \bar{n}_{H A^{\prime}}-k_{R} \bar{n}_{A}, a_{H}, \\
& \bar{j}_{H A} / 2=\bar{j}
\end{aligned}
$$

and Eq. 199 which remains in the original form.

Due to nonlinearities, a consequence of the existence of dimer complexes, the system does not have an analytical solution in a closed form. For the first time it has been possible to obtain approximate analytical solution of this model for steady state using the following approach .

The system consists of linear and nonlinear equations. The linear part in matrix form reads

$$
\{\mathrm{K}\}\{\mathrm{D}\}=\{\mathrm{C}\} \text {, }
$$


where $\{K\}$ is the matrix of kinetic coefficients for the first order processes, given by

$$
\{k\}=\left(\begin{array}{cccc}
-k_{S} / 2 & \left(k_{S} / 2+k_{D}\right) & 0 & -k_{R} a_{H} \\
-\left(k_{S} / 2+k_{D}\right) & k_{S} / 2 & k_{R} a_{H} & 0 \\
-k_{S} / 2 & k_{S} / 2 & 0 & 0 \\
1 & 1 & 1 & 1
\end{array}\right)
$$

and $\{D\}$ is a one dimensional vector of steady state densities given by

$$
\{\mathrm{D}\}=\left(\begin{array}{c}
\bar{n}_{\mathrm{HA}} \\
\bar{n}_{\mathrm{HA}} \\
\bar{n}_{A^{\prime}} \\
\bar{n}_{A^{\prime \prime}}
\end{array}\right),
$$

and the vecor constant $\{C\}$ is equal to

$$
\{c\}=\left(\begin{array}{c}
0 \\
0 \\
\bar{j} \\
N_{O}-2\left(\bar{n}_{H A_{2}^{\prime}}+\bar{n}_{H A_{2}^{\prime \prime}}\right)
\end{array}\right)
$$

The solution of set of Eqs. 205 is as follows:

$$
\begin{aligned}
& \bar{n}_{H A^{\prime}}=-\sigma_{1} \bar{j}+\sigma_{2} N_{0}^{*}, \\
& \bar{n}_{H A^{\prime \prime}}=\sigma_{1} \bar{j}+\sigma_{2} N_{0}^{*}, \\
& \bar{n}_{A^{\prime}}=-\sigma_{3} \bar{j}+\sigma_{4} N_{0}^{*},
\end{aligned}
$$




$$
\bar{n}_{A^{\prime \prime}}=\sigma_{3} \bar{j}+\sigma_{4} N_{0}^{*}
$$

where

$$
\begin{aligned}
& \sigma_{1}=1 / \mathrm{k}_{\mathrm{S}}, \\
& \sigma_{2}=\frac{1}{2(1+1 / \mathrm{h})}, \\
& \sigma_{3}=\frac{1}{\mathrm{~h}}\left(\frac{1}{\mathrm{k}_{\mathrm{S}}}+\frac{1}{\mathrm{k}_{\mathrm{D}}}\right), \\
& \sigma_{4}=\frac{1}{2(1+\mathrm{h})}, \\
& \mathrm{N}_{0}^{*}=\mathrm{N}_{\mathrm{O}}-2\left(\overline{\mathrm{n}}_{\mathrm{HA}}+\overline{\mathrm{n}}_{\mathrm{HA}}{ }_{2}^{\prime}\right),
\end{aligned}
$$

and

$$
h=a_{H} /\left(k_{D} / k_{R}\right)^{2}
$$

The steady state solution indicates that the displacement of densities of the constituents of the transport system is symmetric, with the exception of dimer complexes.

The nonlinear part of the system of equations can be combined to obtain

$$
\bar{j}=\frac{k_{\alpha}}{k_{B}}\left(k^{\prime} \bar{n}_{A^{\prime}}, \bar{n}_{H A^{\prime}}-k^{\prime \prime} \bar{n}_{A^{\prime \prime}} \bar{n}_{H A^{\prime \prime}}\right)-\bar{j}\left(k^{\prime}+k^{\prime \prime}\right) / k_{B} \text {. }
$$

The steady state flux is obtained from the following two equations:

$$
\begin{aligned}
& \bar{j}^{2}\left[\frac{k_{\alpha}}{k_{B}} \frac{1}{h k_{s}}\left(\frac{1}{k_{s}}+\frac{1}{k_{D}}\right)\left(k^{\prime}-k^{\prime \prime}\right)\right] \\
& -\bar{j}\left[1+\left(k^{\prime}+k^{\prime \prime}\right) \frac{1}{k_{B}}+\frac{k_{\alpha}}{k_{B}} \frac{1}{1+h}\left(\frac{1}{k_{D}}+\frac{2}{k_{S}}\right)\left[\frac{N_{O}}{2}-\left(\bar{n}_{H A_{2}^{\prime}}+\bar{n}_{H A_{2}^{\prime \prime}}\right)\right]\right] \\
& +\frac{k_{\alpha}}{k_{B}} h \frac{\left(k^{\prime}-k^{\prime \prime}\right)}{(1+h)^{2}}\left(N_{O}-\left(\bar{n}_{H A_{2}^{\prime}}+\bar{n}_{H_{2}^{\prime \prime}}\right)\right)^{2}=0
\end{aligned}
$$

and 
$\bar{n}_{H_{2}^{\prime}}+\bar{n}_{H A_{2}^{\prime \prime}}=\frac{2 \frac{k_{\alpha}}{k_{B}}\left(\frac{N_{0} / 2}{1+h}\right) h+2 \frac{k_{\alpha}}{k_{B}} \frac{\bar{j}^{2}}{h k_{S}}\left(\frac{1}{k_{S}}+\frac{1}{k_{D}}\right)}{1+2 \frac{\alpha}{k_{B}} \frac{h}{(1+h)^{2}}\left(N_{0}-\left(\bar{n}_{H A_{2}^{\prime}}+\bar{n}_{H_{2}^{\prime \prime}}\right)\right)}$

A considerably simpler expression can be obtained if it is assumed that the density of dimer complexes is small compared to the total density of other constituents. Then, instead of Eq. 199, the conservation equation is

$$
\bar{n}_{\mathrm{HA}^{\prime}}+\bar{n}_{\mathrm{A}^{\prime}}+\bar{n}_{\mathrm{HA}}+\bar{n}_{\mathrm{A}^{\prime \prime}}=\mathrm{N}_{0}
$$

and the flux $\bar{j}$ can be obtained from the quadratic equation given below

$$
\begin{gathered}
\bar{j}^{2}\left[\frac{k_{\alpha}}{k_{B}} \frac{1}{h k_{S}}\left(\frac{1}{k_{S}}+\frac{1}{k_{D}}\right)\left(k^{\prime}-k^{\prime \prime}\right)\right]-\bar{j}\left[1+\left\{\frac{1}{k_{B}}+\frac{k_{\alpha}}{k_{B}} \frac{N_{O} / 2}{1+h}\left(\frac{2}{k_{S}}+\frac{1}{k_{D}}\right)\right\}\left(k^{\prime}+k^{\prime \prime}\right)\right] \\
+\frac{k_{\alpha}}{k_{B}}\left(\frac{N_{0} / 2}{1+h}\right)^{2} h\left(k^{\prime}-k^{\prime \prime}\right)=0
\end{gathered}
$$

Kinetic Limitations

If the densities of constituents do not deviate from the initial equilibrium values when a potential difference is applied across the membrane, then there are no kinetic limitations. If such deviations do occur, then the externally driven flux decreases with time because density gradients of constituents are set up that oppose the net forward flux. From the inspection of the kinetic scheme (Fig. 33) 
follows that, for example, if the right side of membrane is positively biased, there is a net influx of dimer complexes, which results in the buildup of $\mathrm{HA}$ and $A^{-}$on that side. Similarly there is a depletion of constituents at the negatively biased surface. In the following sections we define the conditions under which different kinds of kinetic limitations develop.

In order to define expressions for the kinetic limitation parameters we make the simplifying assumption that

$\frac{1}{k_{S}}\left(\frac{1}{k_{S}}+\frac{1}{k_{D}}\right)\left(\frac{k^{\prime}-k^{\prime \prime}}{1+h} N_{0}\right)^{2} \ll\left[\frac{k^{\prime}+k^{\prime \prime}+k_{B}}{k_{\alpha}}+\frac{k^{\prime}+k^{\prime \prime}}{(1+h)}\left(\frac{1}{k_{D}}+\frac{2}{k_{S}}\right) \frac{N_{0}}{2}\right]^{2}$

Under this condition the physically meaningful root of Eq. 223 for the flux is given by

$\bar{j}=\frac{\frac{h}{(1+h)^{2}}\left(k^{\prime}-k^{\prime \prime}\right)\left(N_{0} / 2\right)^{2}}{\frac{k^{\prime}+k^{\prime \prime}+k_{B}}{k_{\alpha}}+\frac{k^{\prime}+k^{\prime \prime}}{1+h}\left(\frac{1}{k_{D}}+\frac{2}{k_{S}}\right) \frac{N_{O}}{2}}$

\section{Slow Return Pathway}

The kinetic Iimitation occurring due to the slow backflow of HA molecules can be related to the changes in the density of HA on either side of the membrane. These changes are symmetric and at steady state are given by

$$
\frac{\bar{n}_{H A^{\prime \prime}}-\bar{n}_{H A}^{i n}}{n_{H A}^{i n}}=\frac{n_{H A}^{i n}-\bar{n}_{H A^{\prime}}}{n_{H A}^{i n}}=\frac{\frac{k_{\alpha}}{k_{B}} \frac{N_{0}}{1+h} \frac{k_{o}}{k} \sinh \left(\frac{e V}{2 k T}\right)}{1+2\left[\frac{k_{\alpha}}{k_{B}} \frac{N_{o} / 2}{1+h}\left(2 \frac{k_{0}}{k_{S}}+\frac{k_{o}}{k_{D}}\right)+\frac{k_{o}}{k_{B}}\right] \cosh \left(\frac{e V}{2 k T}\right)}
$$


The kinetic limitation parameter $\dddot{E}_{1}$, which is associated with the slow backflow of HA molecules, is defined as the voltage independent part of the numerator of Eq. 226.

$$
E_{1}=\frac{2 k_{\alpha}}{k_{B}} \frac{N_{0}}{1+h} \frac{k_{0}}{k_{s}} \text {. }
$$

Therefore, the greater the $E_{1}$, the greater the deviation of density of neutral molecules HA from the equilibrium value. Eq. 227 indicates that the kinetic limitation is increased (1) by the slow rate of backflow of neutral $\mathrm{HA}$ molecules $\left(\mathrm{k}_{\mathrm{S}}\right.$ compared to $\left.\mathrm{k}_{0}\right)$, (2) by the increase of the total density of uncoupler at the membrane surfaces $\mathrm{N}_{0}$ (3) iy litie increase of the magnitude of the dimer formation constant $k_{\alpha} / k_{\beta}$, and (4) by the increase of $\mathrm{pH}$ of the solution.

\section{Slow Interfacial Process}

The steady state changes of the density of the anions from equilibrium are given by

$$
\frac{\bar{n}_{A^{\prime \prime}}-n_{A}^{i n}}{n_{A}^{i n}}=\frac{n_{A}^{i n}-\bar{n}_{A^{\prime}}}{n_{A}^{i n}}=\frac{\frac{k_{\alpha}}{k_{B}}\left(\frac{k_{o}}{k_{S}}+\frac{k_{o}}{k_{D}}\right) \frac{N_{o}}{1+h} \sinh \left(\frac{e V}{2 k T}\right)}{1+2\left[\frac{k_{\alpha}}{k_{B}} \frac{N / 2}{1+h}\left(\frac{2 k_{o}}{k_{S}}+\frac{k_{o}}{k_{D}}\right)+\frac{k_{o}}{k_{B}}\right] \cosh \left(\frac{e V}{2 k T}\right)}
$$

The voltage independent part of the numerator is

$$
\mathrm{L}=\frac{\mathrm{k}_{\alpha}}{\mathrm{k}_{B}} \frac{\mathrm{k}_{0}}{\mathrm{k}_{S}} \frac{\mathrm{N}_{0}}{1+\mathrm{h}}+\frac{\mathrm{k}_{\alpha}}{\mathrm{k}_{B}} \frac{\mathrm{k}_{0}}{\mathrm{k}_{\mathrm{D}}} \frac{\mathrm{N}_{0}}{1+\mathrm{h}} .
$$


The expression indicates that the displacement of anions from the initial equilibrium results from the slow backflow of HA molecules (term containing $\mathrm{k}_{\mathrm{S}}$ ), as well as from the slow interfacial reaction (term containing $k_{D}$ ). Hence the kinetic limitation parameter $E_{2}$ for the slow interfacial process may be defined as

$$
E_{2}=\frac{k_{\alpha}}{k_{B}} \frac{N_{0}}{1+h} \frac{k_{0}}{k_{D}} .
$$

It indicates that the kinetic limitation is increased ( 1 ) by the slow interfacial reaction ( $k_{D}$ small compared to $\left.k_{0}\right),(2)$ by the increase of the total density of uncoupler $\mathrm{N}_{0},(3)$ by the increase of the magnitude of the dimer formation constant $\mathrm{k}_{\alpha} / \mathrm{k}_{\beta}$, and $(4)$ by the increase of $\mathrm{pH}$ of the solution.

\section{Slow Dimerization Process}

Since the formation of dimers is a process of second order, the positively and negatively biased surfaces of the membrane are considered separately. For the positively biased surface the relative displacement of dimer density from equilibrium is

$$
\frac{\bar{n}_{\mathrm{HA}_{2}}-\mathrm{n}_{\mathrm{HA}}^{\text {in }}}{\mathrm{n}_{\mathrm{HA}}^{\mathrm{in}}}=\frac{\left(\frac{\mathrm{k}_{\alpha}}{\mathrm{k}_{B}} \frac{\mathrm{N}_{0}}{1+\mathrm{h}}\left(\frac{2 \mathrm{k}_{0}}{\mathrm{k}_{\mathrm{S}}}+\frac{\mathrm{k}_{0}}{\mathrm{k}_{\mathrm{D}}}\right)+\frac{2 \mathrm{k}_{0}}{\mathrm{k}_{\beta}}\right) \sinh (\mathrm{eV} / 2 \mathrm{kT})}{1+\left(\frac{\mathrm{k}_{\alpha}}{\mathrm{k}_{B}} \frac{\mathrm{N}_{0}}{1+\mathrm{h}}\left(\frac{2 \mathrm{k}_{0}}{\mathrm{k}_{\mathrm{S}}}+\frac{\mathrm{k}_{0}}{\mathrm{k}_{\mathrm{D}}}\right)+\frac{2 \mathrm{k}_{0}}{\mathrm{k}_{B}}\right) \cosh (\mathrm{eV} / 2 \mathrm{kT})}
$$




$$
+\left(\frac{1+h}{h}\right)^{2} \frac{1}{k_{S}}\left(\frac{1}{k_{S}}+\frac{1}{k_{D}}\right) \frac{\bar{j}^{2}}{\left(N_{0} / 2\right)^{2}}
$$

and for the negatively biased surface it is

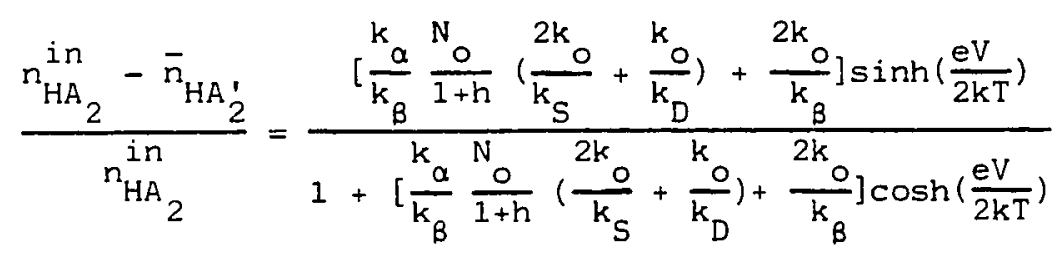

$$
\begin{aligned}
& -\left(\frac{1+h}{h}\right)^{2} \frac{1}{k_{S}}\left(\frac{1}{k_{S}}+\frac{1}{k_{D}}\right) \frac{\bar{j}^{2}}{\left(N_{0} / 2\right)^{2}}
\end{aligned}
$$

Eqs. 231 and 232 indicate the asymmetry in the displacement of dimer density from the equilibrium; the magnitude of the displacement being greater at the positively biased side compared to the opposite side. The average relative displacement of dimer density, taking into account both surfaces, has been used in arriving at the net kinetic limitation parameter $E_{O^{*}}$

$$
E_{O}=\frac{k_{\alpha}}{k_{B}} \frac{N_{O}}{h+1}\left(\frac{2 k_{0}}{k_{S}}+\frac{k_{O}}{k_{D}}\right)+\frac{2 k_{O}}{k_{B}} .
$$

In view of the above result, the remaining term $2 k_{0} / k_{B}$ may be defined as the kinetic Iimitation parameter of the dimerization process;

$$
E_{3}=2 k_{O} / k_{B}
$$

It follows that the net kinetic limitation parameter $E_{O}$ has a rather simple physical meaning. It is the sum of kinetic limitation parameters along the pathways leading to the formation and decomposition of membrane permeable ions. 


$$
E_{0}=E_{1}+E_{2}+E_{3}
$$

In contrast to $E_{3}$, the kinetic limitation parameters in the return and interfacial pathways $E_{1}$ and $E_{2}$ are dependent upon the $\mathrm{pH}$ and the total density of uncoupler adsorbed at the membrane surfaces, $\mathrm{N}_{0^{\circ}}$

When the kinetic limitation due to slow backflow of HA molecules is large (i.e. $\left.E_{1} \geq 1\right)$, it can be distinguished from the other two types of kinetic limitations by the voltage dependence of the normalized membrane conductivity;

$\frac{G(V)}{G(0)}=\frac{\left[1+E_{1} \cosh (e V / 2 k T)-\left(1+2 E_{1} \cosh (e V / 2 k T)+E_{1}^{2}\right)^{\frac{1}{2}}\right] 2\left(1+E_{1}\right)}{(e V / 2 k T) E_{1}^{2} \sinh (e V / 2 k T)}$

For the other two types of kinetic limitations the normalized conductivity is given by

$\frac{G(V)}{G(0)}=\frac{\sinh (e V / 2 k T)}{(e V / 2 k T)} \frac{1+E}{1+E \cosh (e V / 2 k T)}$

where $E=E_{2}$ when the flux is limited by slow interfacial reaction, and $E=E_{3}$ when the $f l u x$ is limited by slow dimerization process. For small values of $E_{1}$ (i.e. $\left.E_{1}<<1\right)$ the normalized membrane conductivity coincides with Eq. 237. The kinetic limitation due to slow dimerization process can be distinguished from that due to slow interfacial reaction by the property that it is independent of the $\mathrm{pH}$ and the uncoupler concentration. Therefore if any one type of kinetic limitation is present to a large extent then it can in principle be identified from the $\mathrm{pH}$ and concentration dependence of the normalized membrane conductivity curves. 
$\underline{\mathrm{pH}}$ of the Conductivity Maximum

The zero voltage conductivity obtained as a limit of $G(V)$ when $\mathrm{V}$ approaches zero is given by

$$
G(0)=k_{0} \frac{k}{k_{B}} \frac{e F}{4 k T} N_{0}^{2} \frac{h}{(1+h)^{2}\left(1+E_{0}\right)}
$$

The $\mathrm{pH}$ corresponding to the maximum conductivity can be obtained from the equation

$$
d E_{0} / d h=\frac{\left(1+E_{0}\right)(1-h)}{h(1+h)} .
$$

It follows that if there are no kinetic limitations or if the membrane flux is limited only by the slow dimerization process, then the conductivity maximum occurs at $\mathrm{pH}$ equal to the $\mathrm{pK}$ a of the uncoupler. For other two types of kinetic limitations the $\mathrm{pH}$ at which the conductivity maximum occurs is given by

$$
\mathrm{pH}_{\operatorname{gmax}}=p K_{a}-\frac{1}{2} \log _{10}\left(1+\frac{\mathrm{k}_{\alpha}}{\mathrm{k}_{B}} \frac{\mathrm{k}_{0}}{\mathrm{k}^{*}} \mathrm{~N}_{0}\right) \text {, }
$$

where $\quad 1 / k^{ \pm}=1 / k_{D}+2 / k_{S}$.

Since the first and the second terms in the above equation are proportional to the kinetic limitation parameters $E_{2}$ and $E_{1}$ respectively, each term dominates for the corresponding type of kinetic limitation. In the presence of these kinetic limitations the $\mathrm{pH}_{\text {gmax }}$ is shifted to a value lower than the $\mathrm{pK}_{\mathrm{a}}$ of the uncoupler. This shift results from the increase of kinetic limitations at high $\mathrm{pH}$ and the consequent decrease of the steady state conductivity from the initial conductivity.

\section{Properties of Transient Flux}

Since the differential equations describing the model are non- 
linear there is no general analytical solution. Hence the predictions of the model can be obtained only from integrating the set of differential Eqs. 191-196, using numerical methods. Several algorithms have been tested, out of which two were found usable.

1. Fourth order Runge-Kutta method was found to be sufficiently accurate but time consuming, especially in cases when some of the reactions were fast and others slow. The reason is that the integration step in such cases has to be sufficiently small in order that the method does not diverge at longer times.

2. Lawrence Livermore Laboratory package called Episode was installed on Laboratory PDP $11 / 03$ minicomputer and its performance was found satisfactory. The main advantage of this program is that its algorithm optimizes the integration step so that the solution takes less time.

Although numerical solution is necessary for the general case it is possible to derive expressions for the relaxation amplitude using approximate solution for the steady state current at various kinetic limitations.

For slow backflow of $\mathrm{HA}$ species the relaxation amplitude is approximately equal to

$$
\begin{aligned}
& \alpha=\frac{E_{1} \sinh ^{2}(e V / 2 k T)}{2\{\cosh (e V / 2 k T)-I\}}-1, \\
& \alpha=\frac{E_{1}}{2}\{\cosh (e V / 2 k T)+1\}-1,
\end{aligned}
$$

provided $E_{1} \gg 1$ and $V$ does not tend to zero. At high voltáges $\alpha$ is Proportional to $\sinh (\mathrm{eV} / 2 \mathrm{kT})$ and $\tanh (\mathrm{eV} / 2 \mathrm{kT})$. The former is due to the 
membrane potential energy barrier and the latter corresponds to saturation effect due to insufficient backflow of HA.

For all other types of kinetic limitations

$\alpha=E_{i} \cosh (e V / 2 k T)$

where $E_{i}$ represents the kinetic limitation parameter. The voltage dependence of $\alpha$ is stronger at high kinetic limitations. The following description of the properties of the transient flux obtained by the numerical methods is in agreement with the predictions based on the above expressions for $\alpha$.

It was found that under all conditions the relaxation amplitude increased and the relaxation time constant decreased with the increase of applied voltage across the membrane. Typical curves are shown in Fig. 34. When the flux is limited by slow dimerization the transients observed were fast and their characteristics were almost independent of the $\mathrm{pH}$ and the concentration. Therefore in the following we describe the $\mathrm{pH}$ and the concentration dependencies of the characteristics of the transient flux when it is limited by the siow backflow of HA molecules and slow interfacial reaction.

These characteristics were obtained from numerical solution at small voltages by fitting the current decay at long times, when the current approached steady state, to a single exponential. These values were then extrapolated to zero voltage to get the zero voltage transient characteristics. Similar procedure is used to analyze experimental results.

In all cases the value of $k_{0}$, the transmembrane ion transloca-
tion rate constant for zero voltage, was normalized to unity. The 


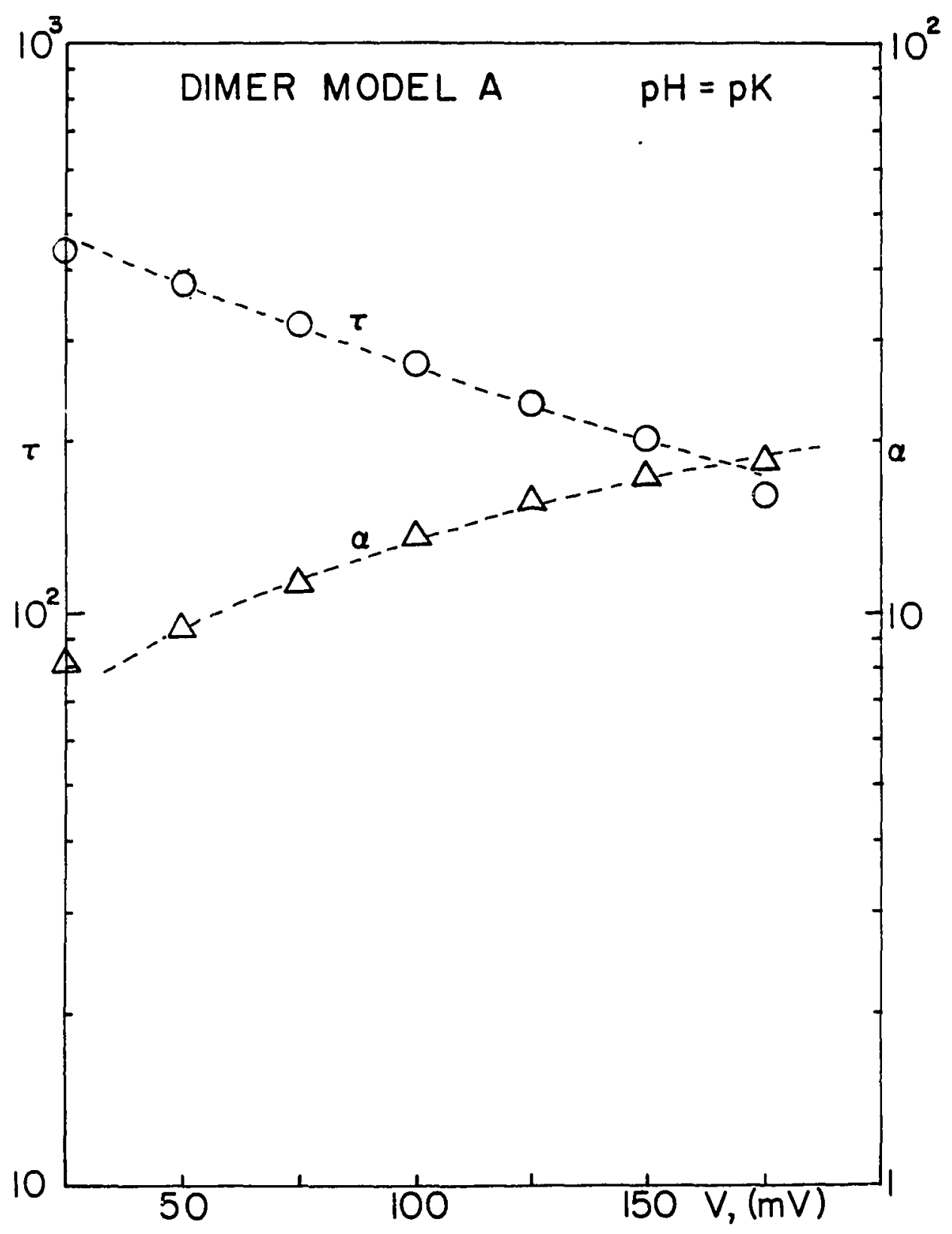

Figure 34. Typical curves showing the decrease of time constant $\tau$ and the increase of relaxation amplitude $\alpha$ with the voltage $\mathrm{V}_{\mathrm{i}}\left(\mathrm{pH}=\mathrm{pK}, \mathrm{N}_{\mathrm{O}}=1, \mathrm{k}_{\mathrm{O}}=1, \mathrm{k}_{\mathrm{S}}=.25 \times 10^{-3}, \mathrm{k}_{\mathrm{D}}=\mathrm{k}_{\mathrm{A}}=.25 \times 10^{-1}\right.$,
$\left.\mathrm{k}_{B}=1 \times 10^{2}, \mathrm{k}_{\alpha}=.1\right)$. 
equilibrium constant $k_{D} / k_{R}$ of monomers was set to one, and the equilibrium constant $\mathrm{k}_{B} / \mathrm{k}_{\alpha}$ of dimers $\mathrm{HA}_{2}^{-}$was fixed at $10^{3}$ such that the dimer density $\bar{n}_{\mathrm{HA}_{2}}=\left(\mathrm{k}_{\alpha} / \mathrm{k}_{\mathrm{B}}\right) \mathrm{n}_{\mathrm{HA}_{\mathrm{A}}} \mathrm{n}_{\mathrm{A}}$, remained small.

Fig. 35 shows the variations of the zero voltage relaxation amplitude $\alpha(0)$ and the zero voltage time constant $\tau(0)$ with pH. The relaxation amplitude increases with $\mathrm{pH}$ for $\mathrm{pH}<\mathrm{pK}$ and attains a steady value at $\mathrm{pH}>\mathrm{pK}$ for both types of kinetic limitations. The time constant increases with $\mathrm{pH}$ for kinetic limitation due to slow proton dissociation and association and when the flux is limited by slow backflow of HA molecules.

Fig. 36 shows the variations of the zero voltage relaxation amplitude $\alpha(0)$ and the zero voltage time constant $\tau(0)$ with concentration. The concentration was changed between .25 and 2 . Under both types of kinetic limitations the relaxation amplitude increased and the time constant decreased with increasing concentration. Fig. 37 shows that the relaxation amplitude is in fact proportional to the concentration.

MODEL 8: DIMER MODEL B

\section{Description of the Model}

For the dimer model A discussed in the preceding section it was assumed that the anion $\mathrm{A}^{-}$adsorbed at the membrane is oriented at the membrane interface in such a way so that it can readily pick up a hydrogen ion from the aqueous phase and be converted into a HA molecule in a single step. It was similarly implied that the HA molecule is favorably oriented at the membrane interface so that it releases a proton directly into the aqueous phase and becomes an anion $\mathrm{A}^{-}$. 


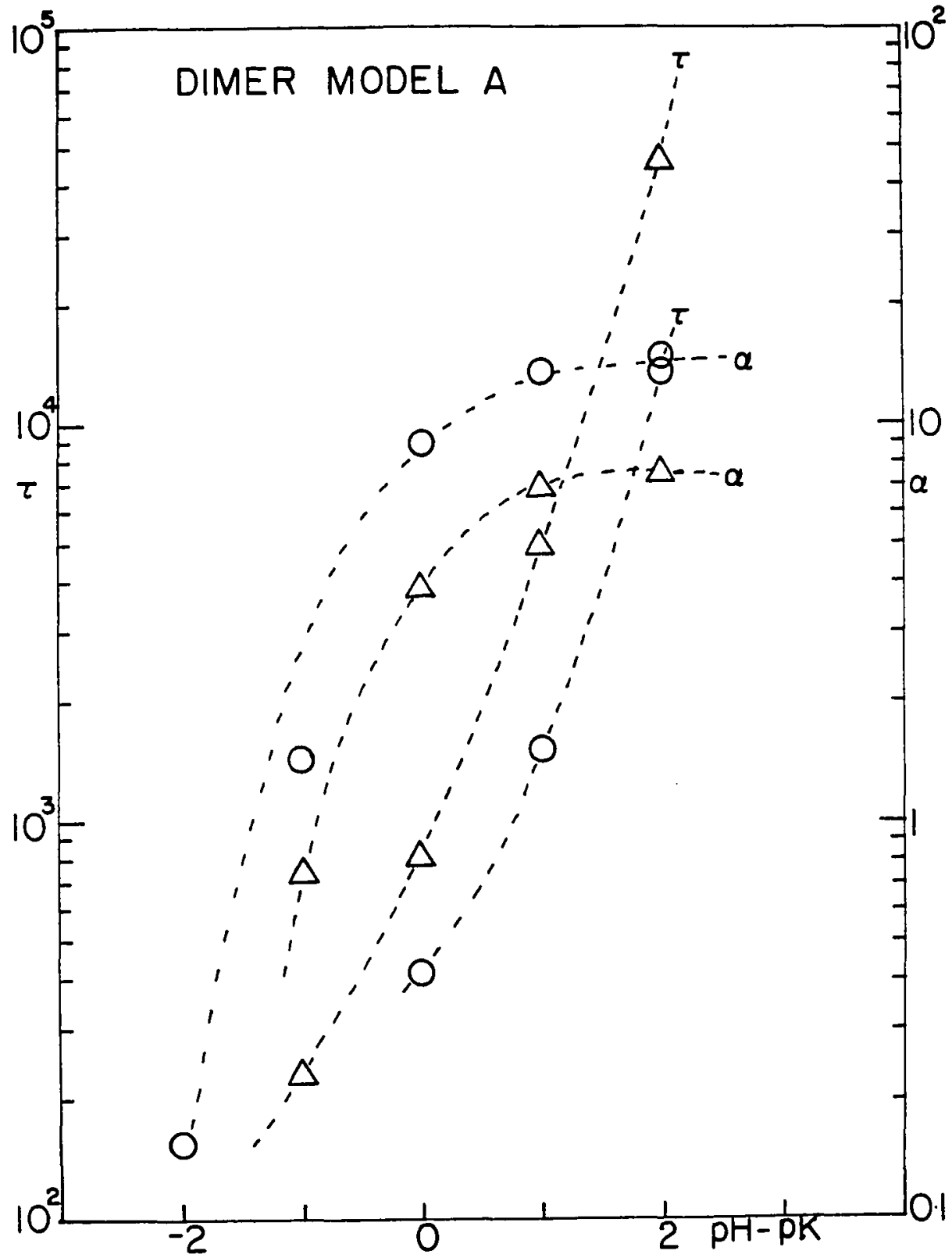

Figure 35. Variation of the zero voltage time constant $\tau(0)$, and the zero voltage relaxation amplitude $\alpha(0)$ with the $\mathrm{pH}$, when the rate limiting step is either the backflow of uncoupler (circles), or the proton association dissociation reaction (triangles). The relaxation amplitude rapidly increases at $\mathrm{pH}<\mathrm{pK}$ and saturates at $\mathrm{pH}>\mathrm{pK}$ (Eqs. 243,244). 


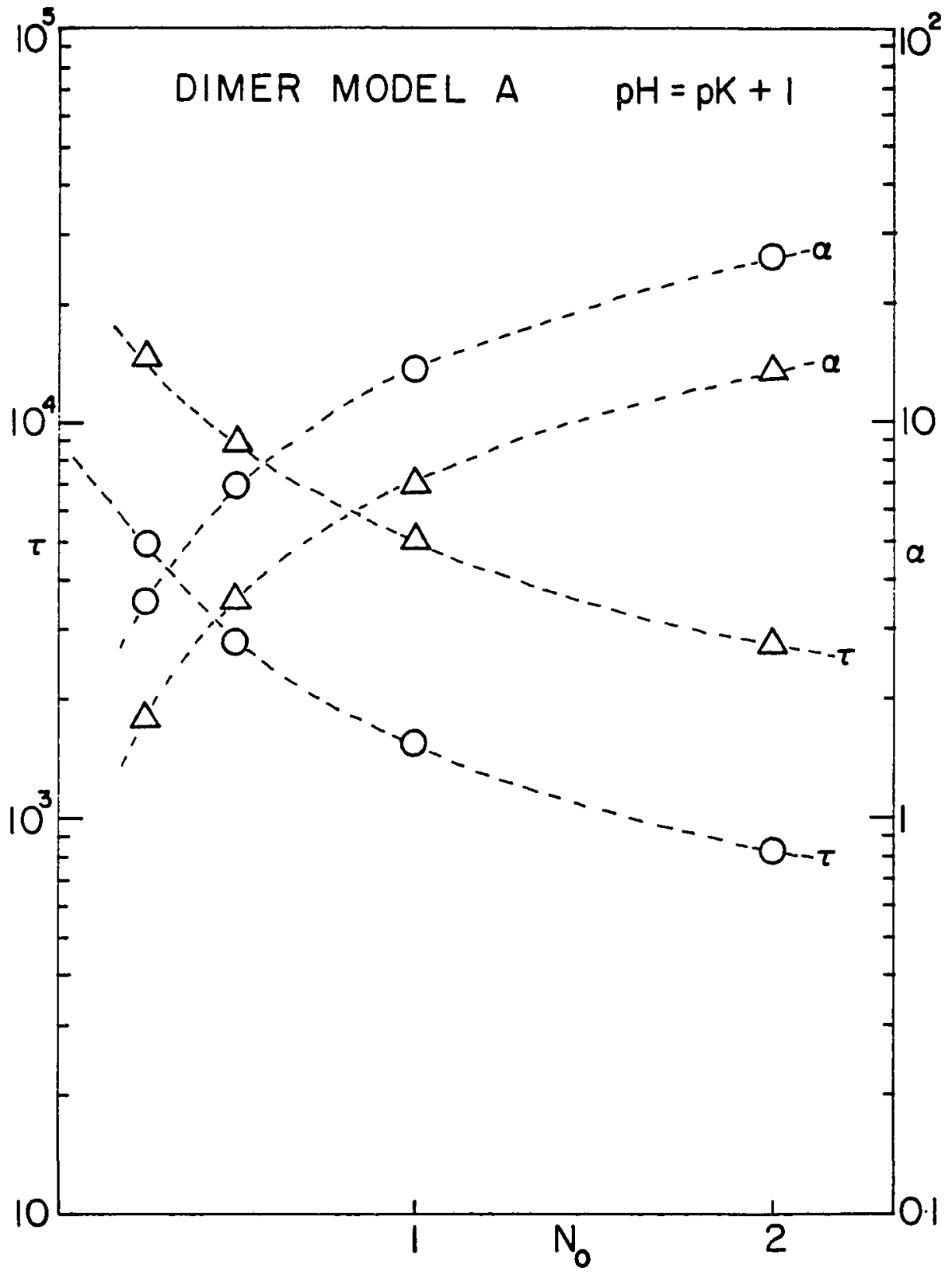

Figure 36. Increase of the zero voltage relaxation amplitude $\alpha(0)$ and decrease of the zero voltage time constant $\tau(0)$ with the uncoupler concentration $\mathrm{N}_{0}$, when the rate limiting step is either the backflow of uncouplers (circles), or proton association dissociation reaction (triangles). $(\mathrm{pH}=\mathrm{pK}+1)$. 


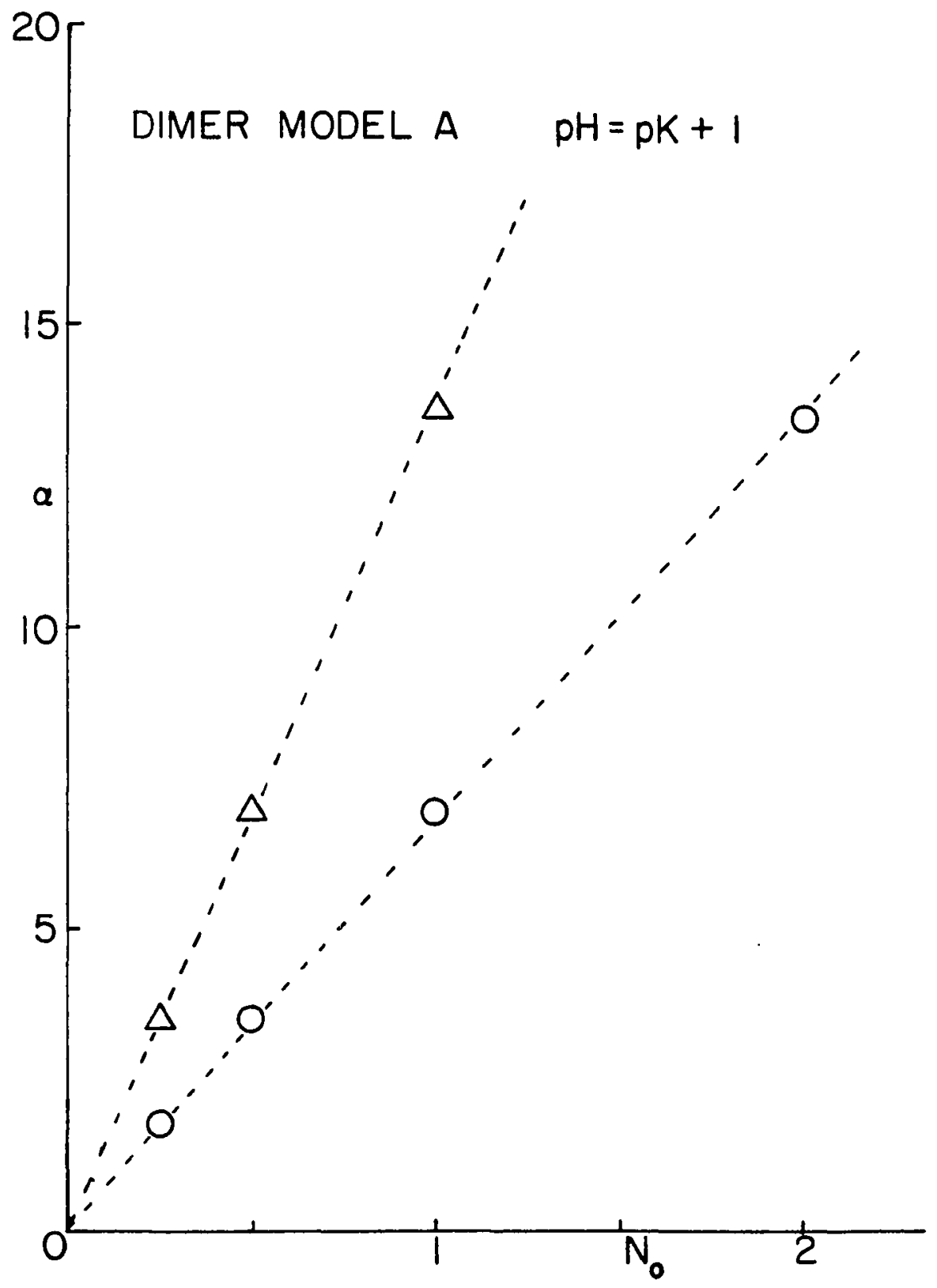

Figure 37. Graph showing that the relaxation amplitude is proportional to the uncoupler concentration $\mathrm{N}_{0}$ (Eq. 244) $(\mathrm{pH}=\mathrm{pK}+1)$, for both the kinetic limitation due to slow kinetics of proton association-dissociation (triangles), and due to slow rate of backflow of HA molecules (circles). 
The model considered in this section is applicable to cases when the molecule involved in the hydrogen ion transfer across the interface is either not properly oriented or it is adsorbed at such a depth so that the rates of direct release and direct association of hydrogen ions are small compared to the rates of desorption of $H A$ and $A^{-}$species.

The model is depicted in Fig. 38. The model differs from the previous one in that it is assumed that the association of hydrogen ion with $\mathrm{A}^{-}$ion and dissociation of $\mathrm{HA}$ molecule takes place in the aqueous phase.

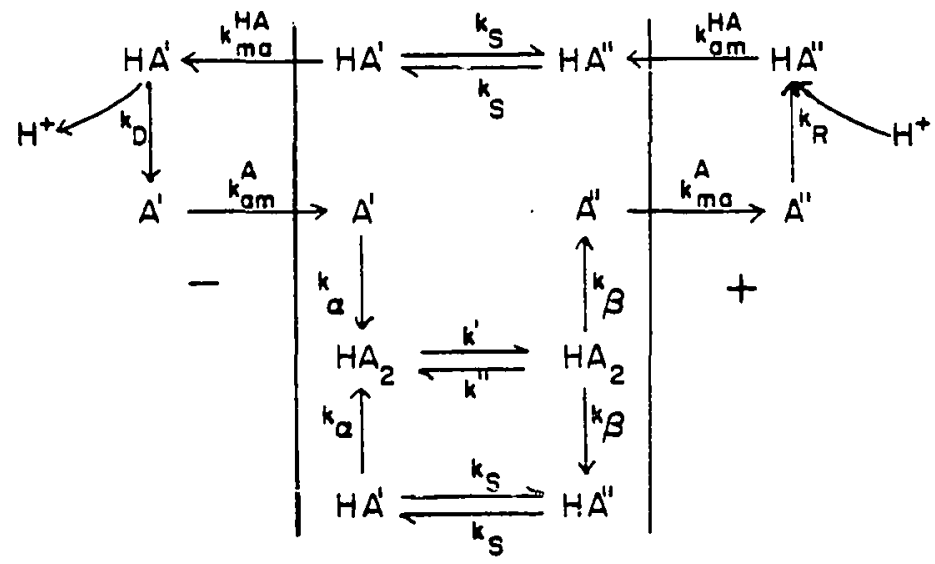

Figure 38. Model 8: Transport scheme for the dimer model $B$.

As in the previous model the charge carrying species is assumed to be the dimer $\mathrm{HA}_{2}^{-}$formed by the reaction $\mathrm{HA}+\mathrm{A}^{-}=\mathrm{HA}_{2}^{-}$Only the neutral HA molecule but not the anion $A^{-}$is assumed to be membrane permeable. The distinctive feature of this model is that the charge is carried across the membrane interface by the anions $A^{-}$. In the aqueous phase tine charge $f \mathbf{i} 0 \mathrm{w}$ is maintained by protons and buffer ions. Assuming that the right hand side is positively biased the charge transport process consists of the following steps. A dimer formed at 
the left hand interface by the combination of $H_{A}$ and $A^{-}$species adsorbed to the interface is driven across the membrane by the externally applied field. On the right interface the dimer dissociates with the release of an anion $A^{-}$and a HA molecule. The HA molecule diffuses back across the membrane while the anion moves into the right hand aqueous phase. In the aqueous phase the anion combines with a proton to produce a HA molecule which then adsorbs at the right hand membrane interface and then diffuses back across the membrane. The HA molecule then desorbs into the left hand aqueous phase and dissociates to release a hydrogen ion and produces an anion $A^{-}$. Due to depletion of $A^{-}$ at the left membrane surface the anion moves back into the left hand membrane surface where it can combine with a HA molecule to form the membrane permeable dimer $\mathrm{HA}_{2}^{-}$, and the cycle is completed. During one transport cycle a proton is moved from the right hand aqueous phase to the left hand aqueous phase.

The model is characterized by the following set of ten differential equations:

$$
\begin{aligned}
& \frac{d}{d t} n_{A_{m}^{\prime}}(t)=k_{B} n_{H A_{2}^{\prime}}-k_{\alpha} n_{A_{m}^{\prime}} n_{H A_{m}^{\prime}}+k_{a m}^{A} n_{A_{a}^{\prime}}(0, t)-k_{m a}^{A} n_{A_{m}^{\prime}} \text {, } \\
& \frac{d}{d t} n_{A_{m}^{\prime \prime}}(t)=k_{B} n_{H A_{2}^{\prime \prime}}-k_{\alpha^{\prime}}^{n_{m}^{\prime \prime}} n_{H A_{m}^{\prime \prime}}+k_{a m}^{A} n_{A_{a}^{\prime \prime}}(0, t)-k_{m a}^{A} n_{A_{m}^{\prime}} \text {, } \\
& \frac{\partial}{\partial t} n_{A_{a}^{\prime}}(x, t)=k_{D} n_{H A_{a}^{\prime}}(x, t)-k_{R^{\prime} n_{a}^{\prime}}(x, t) \cdot a_{H}+D^{A} \frac{\partial^{2}}{\partial x^{2}} n_{A_{a}^{\prime}}(x, t) \\
& +\left\{k_{m a}^{A} n_{A_{m}^{\prime}}-k_{a m A_{a}^{\prime}}^{A}(0, t)\right\} \delta(x) \text {, } \\
& \frac{\partial}{\partial t} n_{A_{a}^{\prime \prime}}(x, t)=k_{D} n_{H A_{a}^{\prime \prime}}(x, t)-k_{R^{\prime \prime}} n_{a}^{\prime \prime}(x, t) \cdot a_{H}+D^{A} \frac{\partial^{2}}{\partial x^{2}} n_{A^{\prime}}(x, t) \\
& +\left\{k_{m a}^{A} n_{A_{m}^{\prime \prime}}-k_{a m}^{A} n_{a}^{\prime \prime}(0, t)\right\} \delta(x-d),
\end{aligned}
$$


$\frac{d}{d t} n_{H A_{m}^{\prime}}(t)=j_{H A}+k_{a m^{n}}^{H A} n_{a}-k_{m a}^{H A} n_{m A_{m}^{\prime}}+k_{B} n_{H A_{2}^{\prime}}-k_{\alpha} n_{H A_{m}^{\prime}} n_{A_{m}^{\prime}}$,

$\frac{d}{d t} n_{H A_{m}^{\prime \prime}}(t)=-j_{H A}+k_{a m}^{H A} n_{H A}^{\prime \prime}-k_{m a}^{H A} n_{H A_{m}^{\prime \prime}}+k_{B} n_{H A_{2}^{\prime \prime}}-k_{\alpha} n_{H A_{m}^{\prime \prime}} n_{A_{m}^{\prime \prime}}$,

$\frac{\partial}{\partial t} n_{H A}(t)=k_{R} n_{A}^{\prime}(x, t) \cdot a_{H}-k_{D} n_{H A}(x, t)+D_{a}^{H A} \frac{\partial^{2}}{\partial x^{2}} n_{H A}^{n_{a}}(x, t)$

$+\left\{k_{m a}^{H A} n_{H A}^{\prime}-k{ }_{m}^{H A} n_{H A},(0, t)\right\} \delta(x)$,

$\frac{\partial}{\partial t} n_{H A}^{\prime \prime}(t)=k_{R} n_{A}^{\prime \prime}(x, t) \cdot a_{H}-k_{D} n_{H A_{a}^{\prime \prime}}(x, t)+D^{H A} \frac{\partial^{2}}{\partial x^{2}} n_{H A}(x, t)$

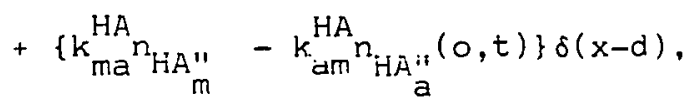

$\frac{d}{d t} n_{H A}(t)=k_{\alpha} n_{H A_{m}} n_{A_{m}^{\prime}}-k_{B} n_{H A}-j$,

$\frac{d}{d t} n_{H A}^{\prime \prime}(t)=k_{\alpha} n_{H A_{m}^{\prime \prime}} n_{A_{m}^{\prime \prime}}-k_{B} n_{H A_{2}^{\prime \prime}}+j$,

where $j=k^{\prime} n_{H_{2}^{\prime}}-k^{\prime \prime} n_{H A_{2}^{\prime \prime}}$,

and

$$
j_{H A}=k_{s}\left(n_{H A_{m}^{\prime \prime}}-n_{H A_{m}^{\prime}}\right) \text {, }
$$

and $\delta(x)=1$ when $x=0$, and $\delta(x)=0$ when $x \neq 0$.

$x=0$ corresponds to the left hand membrane surface and $x=d$ corresponds to the right hand suface. Eqs. $247,248,251$ and 252 contain second partial derivatives of aqueous concentrations $\mathrm{n}_{\mathrm{A}}$ and $\mathrm{n}_{\mathrm{HA}}$ with respect to the position; these terms represent the diffusion of these species in the aqueous phase. It is assumed that the effect of the double layer potential, due to adsorbed charges at the membrane, on the aqueous concentration of anions adjacent to the membrane is negligible.

The above set of differential equations is difficult to solve even by numerical methods. One assumption that greatly simplifies the solution is that the rate of diffusion of $A_{a}$ and $H A_{a}$ species in the 
aqueous phase is negligible compared to the rate of proton association and dissociation. With this assumption the aqueous concentrations of HA and $\mathrm{A}^{-}$become independent of the distance and the terms containing the second partial derivatives can be neglected. In order to convert volume concentrations of membrane transport components into surface densities the aqueous volume concentrations were multiplied by a distance $\gamma$. $\gamma$ is equivalent to thie "reaction layer thickness" and it is defined in the appendix. The simplified set of differential equations is given below.

$$
\begin{aligned}
& \frac{d}{d t} n_{A_{m}^{\prime}}=k_{B} n_{H A_{2}^{\prime}}-k_{\alpha} n_{A_{m}} n_{H A_{m}^{\prime}}+k_{a m}^{A} r n_{A_{a}^{\prime}}-k_{m a^{\prime}}^{A} n_{m}^{\prime} \text {, } \\
& \frac{d}{d t^{\prime \prime}} n_{m}=k_{B} n_{H A_{2}^{\prime \prime}}-k_{\alpha} n_{A_{m}^{\prime \prime}} n_{H A_{m}^{\prime \prime}}+k_{a m}^{A} \gamma_{A_{a}^{\prime \prime}}-k_{m a}^{A} n_{A_{m}^{\prime \prime}} \text {, } \\
& \gamma \frac{d}{d t} n_{A_{a}^{\prime}}=k_{D} \gamma n_{H A_{a}^{\prime}}-k_{R} \gamma n_{A_{a}^{\prime}} a_{H}+k_{m a^{\prime}}^{A} n_{m}^{\prime}-k_{a m}^{A} \gamma n_{A_{a}^{\prime}}, \\
& \gamma \frac{d}{d t} n_{A}^{\prime \prime}=k_{D} \gamma n_{H A_{a}^{\prime \prime}}-k_{R}^{\gamma n_{A_{a}^{\prime \prime}}} a_{H}+k_{m a}^{A} n_{m}^{\prime \prime}-k_{a m}^{A} \gamma n_{A}^{\prime \prime}, \\
& \frac{d}{d t} n_{H A_{m}^{\prime}}=j_{H A}+k_{a m}^{H A} r n_{H A_{a}^{\prime}}-k_{m a}^{H A} n_{H A_{m}^{\prime}}+k_{\beta} n_{H A_{2}^{\prime}}-k_{\alpha} n_{H A_{m}^{\prime}} n_{A_{m}^{\prime}} \text {, } \\
& \frac{d}{d t} n_{H A}^{\prime \prime}=-j_{H A}+k_{a m}^{H A} \gamma n_{H A} A_{a}^{\prime \prime}-k_{m a}^{H A} n_{H A}^{\prime \prime}+k_{B} n_{H A} A_{2}^{\prime \prime}-k_{\alpha} n_{H A}{ }_{m}^{\prime \prime} n_{m}^{\prime \prime},
\end{aligned}
$$

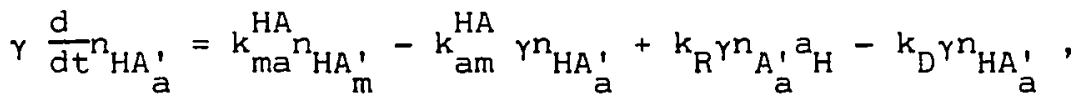

$$
\begin{aligned}
& \gamma \frac{d}{d t} n_{H A}^{\prime \prime}=k_{m a}^{H A} n_{H A}^{\prime \prime}-k_{a m}^{H A} \gamma n_{H A}^{\prime \prime}+k_{R} \gamma n_{A} a_{H}-k_{D} \gamma n_{H A}{ }_{a}^{\prime \prime}, \\
& \frac{d}{d t} n_{H A_{2}^{\prime}}=k_{a} n_{H A_{m}} n_{A_{m}^{\prime}}-k_{B} n_{H A_{2}^{\prime}}-j \text {, }
\end{aligned}
$$


$\frac{d}{d t}{ }_{H A}^{\prime \prime}=k_{\alpha} n_{H A_{m}^{\prime \prime}} n_{A_{m}^{\prime \prime}}-k_{B} n_{H A_{2}^{\prime \prime}}+j$,

where $j$ and $j_{\text {HA }}$ are given by Eqs. 255 and 256 .

Steady State Properties

The steady state properties of the transport model can be obtained by equating the rate equations to zero. Since the set of ten equations obtained is not linearly independent, we replace the tenth equation by the statement of the conservation of mass.

$$
\begin{aligned}
n_{A_{m}^{\prime}}+n_{A_{m}^{\prime \prime}} & +r n_{a}^{\prime}+\gamma n_{a}^{\prime \prime}+n_{H A_{m}^{\prime}}+n_{H A_{m}^{\prime \prime}} \\
& +\gamma n_{H A_{a}^{\prime}}+\gamma n_{H_{a}^{\prime \prime}}+2\left(n_{H A_{2}^{\prime}}+n_{H A_{2}^{\prime \prime}}\right)=N_{C,}
\end{aligned}
$$

where $\mathrm{N}_{0}$ is the surface density of the uncoupler in all forms on both sides of the membrane. The system of equations can be simplified as follows.

$$
\begin{aligned}
& \frac{1}{2} \bar{j}_{H A}=k_{a m}^{A} r \bar{n}_{A_{a}^{\prime}}-k_{m a}^{A} \bar{n}_{A_{m}^{\prime}}, \\
& \frac{1}{2} \bar{j}_{H A}=k_{m a}^{A} \bar{n}_{A_{m}^{\prime \prime}}-k_{a m}^{A} \gamma \bar{n}_{A_{a}^{\prime \prime}}, \\
& \frac{1}{2} \bar{j}_{H A}=k_{a m}^{H A} r \bar{n}_{H A_{a}^{\prime}}-k_{m a}^{H A} \bar{n}_{H A_{m}^{\prime}} \text {, } \\
& \frac{1}{2} \bar{j}_{H A}=k_{m a}^{H A} \bar{n}_{H A_{m}^{\prime \prime}}-k_{a m}^{H A} \gamma \bar{n}_{H A_{a}^{\prime \prime}}, \\
& \frac{1}{2} \bar{j}_{H A}=k_{R} \gamma \bar{n}_{A}^{\prime} a_{H}-k_{D} \gamma \bar{n}_{H A}, \\
& \frac{1}{2} \bar{j}_{H A}=k_{D} \gamma \bar{n}_{H A_{a}^{\prime \prime}}-k_{R} \gamma \bar{n}_{A_{a}^{\prime \prime}} a_{H} \text {, }
\end{aligned}
$$


$\frac{1}{2} \bar{j}_{H A}=k_{B} \bar{n}_{H A}{ }_{2}-k_{\alpha} \bar{n}_{H_{m}} \bar{n}_{A_{m}^{\prime}}$,

$\frac{1}{2} \bar{j}_{H A}=k_{\alpha} \bar{n}_{H A}{ }_{m}^{\prime \prime} \bar{n}_{m}^{\prime \prime}-k_{B} \bar{n}_{H A_{2}^{\prime \prime}}$,

$\frac{1}{2} \bar{j}_{H A}=\bar{j}$,

and Eq. 267, which remains in the original form.

This system of equations does not have an analytical solution in a closed form because it is nonlinear. An approximate solution has been found using the following approach. The system of equations can be divided into a linear and a nonlinear part. The linear part, written in matrix form, is

$\{K\}\{D\}=\{C\}$,

where $\{\mathrm{K}\}$ is the matrix of the kinetic coefficients for the first order processes and is given by

$\{K\}=$

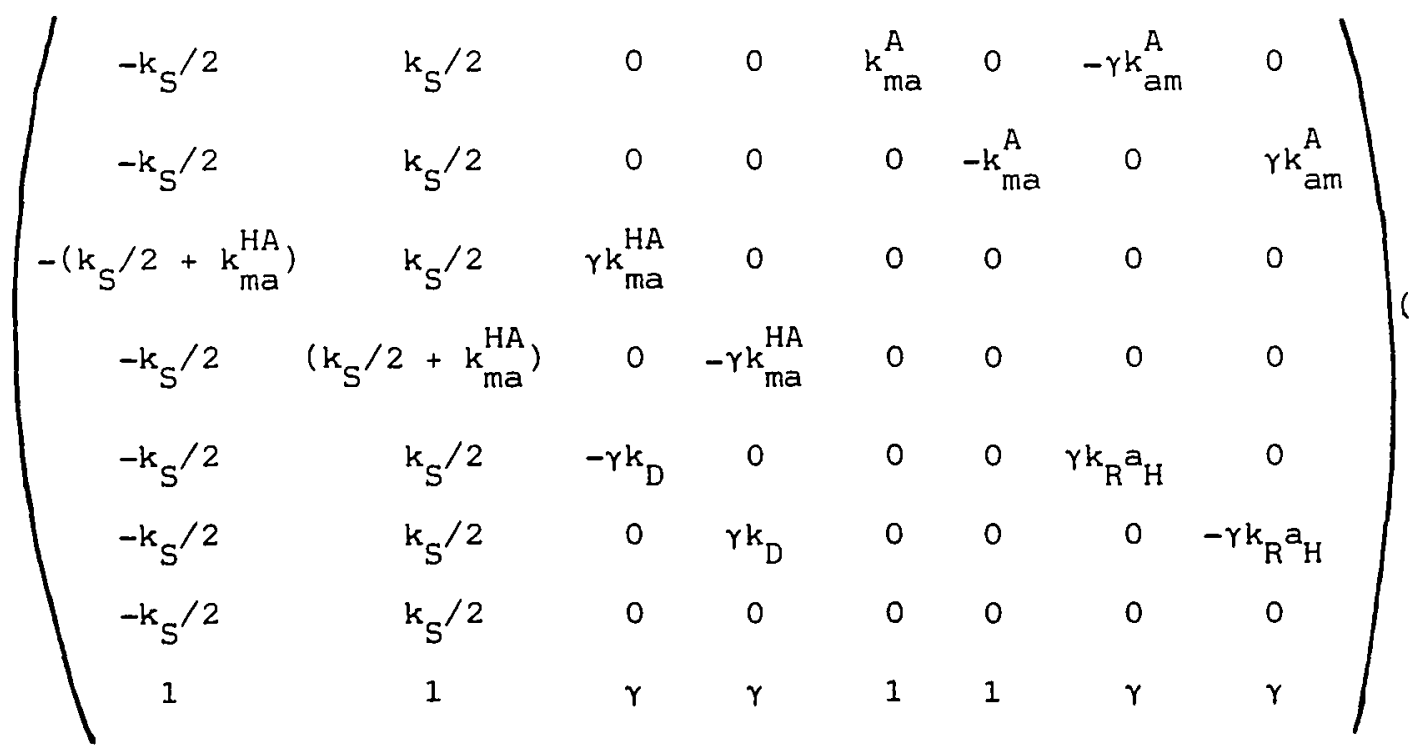


\{D\} is a one dimensional vector of steady state densities:

$\{D\}=\left(\begin{array}{c}\bar{n}_{H A}^{\prime} \\ \bar{n}_{m A^{\prime \prime}} \\ \bar{n}_{\mathrm{HA}}^{\prime} \\ \bar{n}_{\mathrm{HA}}^{\prime \prime} \\ \bar{n}_{\mathrm{A}}^{\prime} \\ \bar{n}_{\mathrm{m}}^{\prime \prime} \\ \bar{n}_{\mathrm{m}}^{\prime} \\ \bar{n}_{\mathrm{A}}^{\prime \prime} \\ \bar{a}^{\prime}\end{array}\right)$

and the vector constant $\{C\}$ is equal to

$\{c\}=\left|\begin{array}{c}0 \\ 0 \\ 0 \\ 0 \\ 0 \\ 0 \\ \bar{j} \\ \mathrm{~N}_{0}-2\left(\bar{n}_{H A_{2}^{\prime}}+\bar{n}_{H A_{2}^{\prime \prime}}\right)\end{array}\right|$

(280)

Solution of this system for the membrane bound components is as follows;

$$
\begin{aligned}
& \bar{n}_{\mathrm{HA}}=-\sigma_{1} \bar{j}+\sigma_{2} \mathrm{~N}_{0}^{*} \text {, } \\
& \bar{n}_{\mathrm{HA}}=\sigma_{1} \bar{j}+\sigma_{2} N_{0}^{k} \text {, } \\
& \bar{n}_{A_{m}^{\prime}}=-\sigma_{3} \bar{j}+\sigma_{4} N_{0}^{*} \text {, } \\
& \bar{n}_{A_{m}^{\prime \prime}}=\sigma_{3} \bar{j}+\sigma_{4} N_{0}^{*} \text {, }
\end{aligned}
$$

where $N_{0}^{*}=N_{0}-2\left(\bar{n}_{H A_{2}^{\prime}}+\bar{n}_{H_{2}^{\prime \prime}}\right)$, 


$$
\sigma_{1}=\left(k_{S}\right)^{-1}
$$

$$
\sigma_{2}=\frac{1}{2}\left[\left(1+\frac{k_{m a}^{H A}}{k_{a m}^{H A}}\right)+\frac{k_{m a}^{H A}}{k_{a m}^{H A}} \quad \frac{1}{h}\left(1+\frac{k_{a m}^{A}}{k_{m a}^{A}}\right)\right]^{-1},
$$

$$
\sigma_{3}=\frac{1}{k_{m a}^{A}}+\frac{k_{a m}^{A}}{k_{m a}^{A}}\left(\frac{1}{h k_{a m}^{H A}}+\frac{k_{m a}^{H A}}{k_{a m}^{H A}} \frac{1}{h k_{S}}+\frac{1}{k_{R^{a} H}}\right),
$$

$$
\sigma_{4}=\frac{1}{2}\left[\left(1+\frac{k_{m a}^{A}}{k_{a m}^{A}}\right)+h \frac{k_{m a}^{A}}{k_{a m}^{A}}\left(1+\frac{k_{a m}^{H A}}{k_{m a}^{H A}}\right)\right]^{-1},
$$

and where $\quad h=\frac{k_{R} a_{H}}{k_{D}}=10^{-\left(p H-p k_{a}\right)}$.

The nonlinear part of the system of equations can be combined to get

$$
\bar{j}=\frac{k_{\alpha}}{k_{B}}\left(k^{\prime} n_{A_{m}^{\prime}} n_{H A_{m}^{\prime}}-k^{\prime \prime} n_{A_{m}^{\prime \prime}} n_{H A_{m}^{\prime \prime}}\right)-\bar{j}\left(\frac{k^{\prime}+k^{\prime \prime}}{k_{B}}\right) .
$$

Solution of the system is greatly simplified if it is assumed that the density of dimer complexes is small compared to the total density of the other constituents:

$$
\text { i.e. } 2\left(\mathrm{n}_{\mathrm{HA}}{ }_{2}+\mathrm{n}_{\mathrm{HA}}{ }_{2}\right) \ll \mathrm{N}_{\mathrm{O}} \text {. }
$$

Then the steady state flux is given by the solution of the quadratic equation

$$
\begin{gathered}
\bar{j}^{2} \sigma_{1} \sigma_{3}\left(k^{\prime}-k^{\prime \prime}\right)-\bar{j}\left[\left(\sigma_{1} \sigma_{4}+\sigma_{2} \sigma_{3}\right)\left(k^{\prime}+k^{\prime \prime}\right) N_{0}\right. \\
\left.+\frac{k_{B}+k^{\prime}+k^{\prime \prime}}{k_{\alpha}}\right]+\sigma_{2} \sigma_{4}\left(k^{\prime}-k^{\prime \prime}\right) N_{0}^{2}=0 .
\end{gathered}
$$




\section{Kinetic Limitations}

Kinetic limitations are manifested by the development of density gradients, which oppose the forward flux and cause the steady state flux to be smaller than the initial flux. Assuming that the right hand side of the membrane is positively biased with respect to the left hand side, the resultant flux will cause a buildup of membrane transport constituents on the right hand side and their depletion on the left hand side. In the following we define the conditions under which different kinetic limitations develop.

In order to define expressions for the kinetic limitation parameters we make the simplifying assumption that

$$
\begin{gathered}
\bar{j}_{0} \ll \sqrt[1]{2} \sqrt{\frac{k_{\alpha}}{k_{B}} n_{H A_{2}}^{i n}}\left[\left(\sqrt{\frac{x_{H A}}{x_{A_{m}}}} \cdot n_{A_{m}}^{i n}+\sqrt{\frac{x_{m}}{x_{H A}}} \cdot n_{H A}^{i n}\right)\left(k^{\prime}+k^{\prime \prime}\right)\right. \\
\left.+\frac{\bar{j}\left(k_{B}+k^{\prime}+k^{\prime \prime}\right)}{k_{\alpha} \sqrt{x_{A_{m}}-x_{H A}}}\right] .
\end{gathered}
$$

where

$$
x_{A_{m}}=\bar{n}_{A_{m}^{\prime \prime}}-n_{A_{m}^{i n}}^{i n}=n_{m}^{i n}-\bar{n}_{A_{m}^{\prime}}
$$

and

$$
x_{\mathrm{HA}}=\bar{n}_{\mathrm{HA}}-n_{\mathrm{HA}}^{\text {in }}=n_{\mathrm{HA}}^{\text {in }}-\bar{n}_{\mathrm{HA}},
$$

so that the steady state $f$ lux is given by

$$
\bar{j}=\frac{\sigma_{2} \sigma_{4}\left(k^{\prime}-k^{\prime \prime}\right) N_{0}^{2}}{\left(\sigma_{1} \sigma_{4}+\sigma_{2} \sigma_{3}^{\prime}\left(k^{\prime}+k^{\prime \prime}\right) N_{0}+\frac{k_{B}+k^{\prime}+k^{\prime \prime}}{k_{\alpha}}\right.} .
$$

The kinetic limitation occurring due to the slow backflow of HA molecules can be related to the changes of density of the membrane bound $\mathrm{HA}$ on either side of the membrane. These changes are symmetric and are given by 


$$
\begin{aligned}
& \frac{\bar{n}_{H_{m}^{\prime \prime}}-n_{H A}^{i n}}{n_{H A}^{i n}}=\frac{n_{H A}^{i n}-\bar{n}_{H A}{ }_{m}}{n_{H A}^{i n}}=\frac{\sigma_{1} \bar{j}}{\sigma_{2} N_{0}} \\
& =\frac{\sigma_{1} \sigma_{4} 2 k_{0} \sinh \left(\frac{e V}{2 k T}\right) N_{0}}{\left\{\left(\sigma_{1} \sigma_{4}+\sigma_{2} \sigma_{3}\right) N_{0}+\frac{1}{k_{\alpha}}\right\} 2 k_{0} \cosh \left(\frac{e V}{2 k T}\right)+\frac{k_{B}}{k_{\alpha}}} .
\end{aligned}
$$

We define the kinetic limitation parameter $E_{1}$, corresponding to slow backflow of $\mathrm{HA}$, as the voltage independent part of the numerator of Eq. 297.

$$
\mathrm{E}_{1}=4 \frac{\mathrm{k}_{\alpha}}{\mathrm{k}_{B}} \mathrm{k}_{0} \sigma_{1} \sigma_{4} \mathrm{~N}_{0}=4 \frac{\mathrm{k}_{\alpha}}{\mathrm{k}_{B}} \frac{\mathrm{k}_{0}}{\mathrm{k}_{\mathrm{S}}} \mathrm{n}_{\mathrm{A}_{\mathrm{m}}}^{\mathrm{in}} .
$$

The steady state displacements of densities of membrane bound anions $A_{m}^{-}$from the equilibrium are symmetric and are given by

$$
\begin{aligned}
\frac{\bar{n}_{A_{m}^{\prime \prime}}-n_{A_{m}}^{i n}}{n_{A_{m}}^{i n}} & =\frac{n_{A_{m}}^{i n}-\bar{n}_{A_{m}^{\prime}}}{n_{A_{m}^{i n}}^{i n}}=\frac{\sigma_{3} \bar{j}}{\sigma_{4^{N}}^{N}} \\
& =\frac{\sigma_{2} \sigma_{3} 2 k_{0} \sinh \left(\frac{e V}{2 k T}\right) N_{O}}{\left\{\left(\sigma_{1} \sigma_{4}+\sigma_{2} \sigma_{3}\right) N_{0}+\frac{1}{k_{\alpha}}\right\} 2 k_{0} \cosh \left(\frac{e V}{2 k T}\right)+\frac{k_{B}}{k_{\alpha}}}
\end{aligned}
$$

The voltage independent part of the numerator is

$$
\begin{aligned}
L & =2 \frac{k_{\alpha}}{k_{B}} k_{0} \sigma_{2} \sigma_{3}{ }^{N}=2 \frac{k_{\alpha}}{k_{B}} k_{0} \sigma_{3} n_{H A}^{i n} \\
& =2 \frac{k_{m}}{k_{B}} k_{0}\left[\frac{n_{H A}^{i n}}{k_{m a}^{A}}+\frac{n_{m}^{i n}}{k_{m a}^{H A}}+\frac{n_{m}^{i n}}{k_{S}}+\frac{k_{a m}^{H A}}{k_{m a}^{H A}} \frac{n_{m}^{i n}}{k_{D}}\right] .
\end{aligned}
$$

This expression shows that the density displacements of membrane bound anions from equilibrium are caused by the following processes:

(1) slow backflow of $\mathrm{HA}$ molecules (the term containing $\mathrm{k}_{\mathrm{S}}$ ), (2) slow hydrogen ion association-dissociation (the term containing $k_{D}$ ), 
(3) slow interfacial transfer of $\mathrm{A}^{-}$(the term containing $\mathrm{k}_{\mathrm{ma}}^{\mathrm{A}}$ ),

(4) slow interfacial transfer of HA (the second term).

Thus the kinetic limitation parameters contained in the expression j00 are defined as follows.

For the slow rate of hydrogen ion association-dissociation,

$E_{2}=2 \frac{k_{\alpha}}{k_{B}} \frac{k_{0}}{k_{D}} \frac{k_{a m}^{H A}}{k_{m a}^{H A}} n_{A_{m}^{i n}}^{i n}$.

For the slow rate of interfacial transfer of anions,

$E_{4}=2 \frac{k_{\alpha}}{k_{B}} \frac{k_{0}}{k_{m a}^{A}} n_{H A}^{i n}$.

For the slow interfacial transfer of HA molecules,

$E_{5}=2 \frac{k_{\alpha}}{k_{B}} \frac{k_{0}}{k_{m a}^{H A}} n_{A_{m}}^{i n}$.

The kinetic limitation parameter for the slow backflow of HA molecules (third term in Eq. 300) is already defined by Eq. 298.

Since the formation of dimers is a process of second order, the relative displacement of dimer density from equilibrium is different at either side of the membrane. For the positively biased side of the membrane,

$$
\frac{\bar{n}_{H_{2}^{\prime \prime}}-n_{H A_{2}}^{i n}}{n_{H A_{2}}^{i n}}=\frac{E_{0} \sinh \left(\frac{e V}{2 k T}\right)}{1+E_{0} \cosh \left(\frac{e V}{2 k T}\right)}+\frac{\sigma_{1} \sigma_{3}}{\sigma_{2} \sigma_{4}} \frac{\bar{j}^{2}}{N_{0}^{2}}
$$

where $\quad E_{0}=4 \frac{k_{\alpha}}{k_{B}} \frac{k_{o}}{k_{S}} n_{A_{m}^{i n}}^{i n}+2 \frac{k_{\alpha}}{k_{B}} \frac{k_{m a}^{H A}}{k_{m a}^{H A}} \frac{k_{o}}{k_{D}} n_{A_{m}}^{i n}+2 \frac{k_{\alpha}}{k_{B}} \frac{k_{o}}{k_{m a}^{A}} n_{H A_{m}^{i n}}^{i n}$ 


$$
+2 \frac{k_{\alpha}}{k_{B}} \frac{k_{o}}{k_{m a}^{H A}} n_{A_{m}^{i n}}^{i n}+2 \frac{k_{0}}{k_{B}} .
$$

For the negatively biased side of the membrane the relative displacement of the dimer density from equilibrium is

$$
\frac{n_{\mathrm{HA}_{2}}^{\text {in }}-\bar{n}_{\mathrm{HA}}{ }_{2}}{n_{\mathrm{HA}}^{\text {in }}}=\frac{E_{0} \sinh \left(\frac{\mathrm{eV}}{2 \mathrm{kT}}\right)}{1+E_{0} \cosh \left(\frac{\mathrm{eV}}{2 \mathrm{kT}}\right)}-\frac{\sigma_{1} \sigma_{3}}{\sigma_{2} \sigma_{4}} \frac{\bar{j}^{2}}{\mathrm{~N}_{0}^{2}} .
$$

The steady state displacement of dimers from equilibrium is greater at the positively biased side than the negatively biased side. The average relative displacement of dimer density, taking into account both surfaces, has been used in arriving at the total kinetic limitation parameter $E_{0}$; this is given by Eq. 305. In this expression one can identify the kinetic limitation parameters $E_{1}, E_{2}, E_{4}$ and $E_{5}$ defined previously. The remaining term could therefore be identified as the kinetic limitation parameter for the dimerization process;

$E_{3}=2 \frac{k_{0}}{k_{B}}$.

It follows that the net kinetic limitation parameter $E_{0}$ has a rather simple physical meaning. It is the sum of kinetic limitation parameters along the pathways leading to the formation and decomposition of membrane permeable ions.

$$
E_{0}=E_{1}+E_{2}+E_{3}+E_{4}+E_{5} \text {. }
$$

In contrast to $\mathrm{E}_{3}$, the other kinetic limitation parameters are depen- 
dent upon the $\mathrm{pH}$ and the total density of uncoupler $\mathrm{N}_{0}$. The initial densities $n_{A_{m}}^{\text {in }}$ and $n_{\mathrm{HA}}^{\text {in }}$ are given by

$n_{A_{m}}^{\text {in }}=\frac{N_{0} / 2}{\left(1+\frac{k_{m a}^{A}}{k_{a m}^{A}}\right)+h \frac{k_{m a}^{A}}{k_{a m}^{A}}\left(1+\frac{k_{a m}^{H A}}{k_{m a}^{H A}}\right)}$,

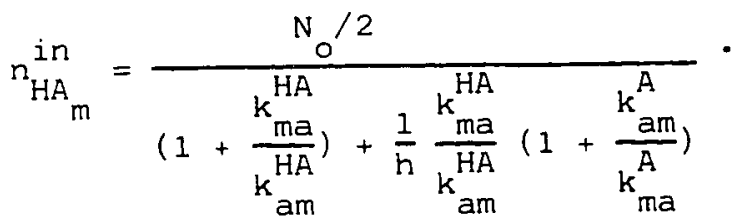

When the kinetic limitation due to slow backflow of HA molecules is large (i.e. $E_{1} \gg 1$ ), it can be recognized from the voltage dependence of the normalized membrane conductivity;

$\frac{G(V)}{G(0)}=\frac{\left[1+E_{1} \cosh \left(\frac{e V}{2 k T}\right)-\left(1+2 E_{1} \cosh \left(\frac{e V}{2 k T}\right)+E_{1}^{2}\right)^{\frac{1}{2}}\right] 2\left(1+E_{1}\right)}{\left(\frac{e V}{2 k T}\right) E_{1}^{2} \sinh \left(\frac{e V}{2 k T}\right)}$.

For all the other types of kinetic limitations the normalized conductivity is

$\frac{G(V)}{G(0)}=\frac{\sinh (e V / 2 k T)}{(e V / 2 k T)} \frac{1+E}{1+E \cdot \cosh (e V / 2 k T)}$,

where $E$ is given by the corresponding expression for each type of kinetic limitation (Eqs. 301-303, 307). For small values of $E_{1}\left(E_{1}<<1\right)$, the normalized membrane conductivity coincides with that predicted for other types of kinetic limitation (Eq. 312).

Some kinetic limitations can be recognized by the $\mathrm{pH}$ and concentration dependence of $G(V) / G(0)$. For example, the kinetic limitation due to slow dimerization can be identified by the fact that it is neither $\mathrm{pH}$ nor concentration dependent. The kinetic limitations due to 
slow proton association-dissociation and that due to slow interfacial transfer of $\mathrm{HA}$, increase with increasing $\mathrm{pH}$ and increasing uncoupler concentration, and for this reason, they cannot be distinguished from each other. In contrast, the kinetic limitation due to slow interfacial transfer of $A^{-}$can be identified by the $p H$ dependence of $G(V) / G(0)$, because the kinetic limitation parameter $E_{4}$ decreases with the $\mathrm{pH}$.

Table II shows the dependence of various types of kinetic limitations in dimer models $\mathrm{A}$ and $\mathrm{B}$ on aqueous $\mathrm{pH}$ and uncoupler concentration.

pH of the Conductivity Maximum

The zero voltage conductivity obtained as a limit of $G(V)$ when $\mathrm{V}$ approaches zero is given by

$$
G(0)=F \frac{k_{\alpha}}{k_{B}} \frac{e k_{0}}{k T} N_{0}^{2} \frac{\sigma_{2} \sigma_{4}}{1+E_{0}} \text {, }
$$

where $E_{0}$ represents the net kinetic limitation parameter. The pH corresponding to the maximum conductivity can be obtained from the equation

$$
\frac{d E}{d h}=\frac{\left(1+E_{0}\right)(B-h)}{h(B+h)} \text {, }
$$

where $\quad B=\left(1+k_{a m}^{A} / k_{m a}^{A}\right) /\left(1+k_{a m}^{H A} / k_{m a}^{H A}\right)$.

It follows that if there are no kinetic limitations or if the membrane flux is limited only by the slow dimerization process, then the conductivity maximum occurs at a $\mathrm{pH}=\mathrm{pH}_{\text {gmax }}$ given by

$$
\mathrm{pH}_{\operatorname{gmax}}=\mathrm{pK} a \mathrm{a}-\log _{10} \mathrm{~B}
$$

The model predicts that it is possible to have the conductivity maximum at a $\mathrm{pH}$ different from the $\mathrm{pK}_{\mathrm{a}}$ of the uncoupler even in the absence of 
TABLE II

DEPENDENCE OF VARIOUS TYPES OF KINETIC LIMITATIONS IN DIMER MODELS A AND B ON AQUEOUS PH AND UNCOUPLER CONCENTRATION

\begin{tabular}{|c|c|c|c|c|}
\hline $\begin{array}{l}\text { Applic } \\
\text { to }\end{array}$ & cable & $\begin{array}{l}\text { Kinetic Limitation } \\
\text { due to }\end{array}$ & $\begin{array}{l}\text { Kinetic } \\
\text { dependence } \\
\text { on } \mathrm{pH}\end{array}$ & $\begin{array}{l}\text { Kinetic } \\
\text { dependence on } \\
\text { concentration }\end{array}$ \\
\hline dimer & models & dimerization & $\mathrm{pH}$ & concentration \\
\hline$A$ and & B & & independent & independent \\
\hline dimer & models & hydrogen ion & increases & increases with \\
\hline$A$ and & $\mathrm{B}$ & association/dissociation & with $\mathrm{pH}$ & concentration \\
\hline dimer & models & backflow & increases & increases with \\
\hline$A$ and & B & of $\mathrm{HA}$ & with $\mathrm{pH}$ & concentration \\
\hline dimer & model & interfacial transfer & increases & increases with \\
\hline B & & of $\mathrm{HA}$ & with $\mathrm{pH}$ & concentration \\
\hline dimer & model & interfacial transfer & decreases & increases with \\
\hline B & & of $\mathrm{A}^{-}$ & with $\mathrm{pH}$ & oncentration \\
\hline
\end{tabular}


any kinetic limitations. The amount and the direction of the shift of $\mathrm{pH}_{\text {gmax }}$ from the $\mathrm{pK}_{\mathrm{a}}$ is determined by the partition coefficients of $\mathrm{A}^{-}$ and HA species in the membrane.

For the other types of kinetic limitations, with the exception of the one due to slow interfacial transfer of anions $A^{-}$, the corresponding $\mathrm{pH}_{\text {gmax }}$ takes the form

$$
\mathrm{pH}_{\text {gmax }}=\left(\mathrm{pK}_{\mathrm{a}}-\log _{10} \mathrm{~B}\right)-\frac{1}{2} \log _{10}(1+\mathrm{x} / \mathrm{B}) \text {, }
$$

where $\quad x=N_{0} \frac{k_{\alpha} k_{0} k_{a m}^{A} / k_{m a}^{A}}{k_{B}\left(1+k_{a m}^{H A} / k_{m a}^{H A}\right)}\left(\frac{2}{k_{S}}+\frac{k_{a m}^{H A}}{k_{m a}^{H A}} \frac{1}{k_{D}}+\frac{1}{k_{m a}^{H A}}\right)$.

Since the first, second, and third terms in the above equation are Froportional to the kinetic limitation parameters $E_{1}, E_{2}$, and $E_{5}$ respectively, each term becomes predominant at the corresponding type of kinetic limitation. In the presence of these kinetic limitations the $\mathrm{pH}_{\text {gmax }}$ is shifted to a $\mathrm{pH}$ lower than the value of $\mathrm{pH}_{\text {gmax }}$ in the absence of kinetic limitations. This shift is the consequence of the increase of kinetic Iimitations with the $\mathrm{pH}$ and the subsequent decrease of steady state conductivity from the initial conductivity at high $\mathrm{pH}$.

For the flux limited by the slow interfacial transfer of anions $\mathrm{A}^{-}$the maximum conductivity occurs at a $\mathrm{pH}$ given by

$$
\mathrm{pH}_{\text {gmax }}=\left(p k_{a}-\log _{10} B\right)+\frac{1}{2} \log _{10}(I+Y)
$$

where $Y=N_{0} \frac{k_{0} k_{\alpha} k_{a m_{m a}^{H A} / k_{m A}^{H A}}^{A} k_{m a}^{A}\left(1+k_{a m}^{H A} / k_{m a}^{H A}\right)}{k_{m a}^{A}}$.

$Y$ is proportional to the corresponding kinetic limitation parameter $E_{4^{*}}$ The p! gmax for this kinetic limitation is shifted to a $\mathrm{pH}$ higher than 
the value of $\mathrm{pH}_{\text {gmax }}$ in the absence of kinetic limitations. This shift results from the increase of kinetic limitations with decreasing $\mathrm{pH}$ and the subsequent decrease of steady state conductivity from the initial conductivity at low $\mathrm{pH}$.

\section{Properties of Transient Flux}

There is no general analytical solution to the differential equations describing the model because the equations are nonlinear. The distribution of densities of constituents as a function of time can be obtained only by integrating the set of differential Eqs. 257-266.

However it is possible to derive approximate expressions for the relaxation amplitude $\alpha$ for individual kinetic limitations using expressions for the steady state current obtained for these kinetic limitations. For slow backflow of $\mathrm{HA}$ species the relaxation amplitude is approximately equal to

$$
\begin{aligned}
& \alpha \simeq \frac{E_{1} \sinh ^{2}(e V / 2 k T)}{2 \cosh (e V / 2 k T)-1}-1, \\
& \alpha \simeq \frac{E_{1}}{2}\{\cosh (e V / 2 k T)+1\}-1,
\end{aligned}
$$

provided $E_{1} \gg 1$ and $V$ does not tend to zero. At high voltages a is proportional to $\sinh (\mathrm{eV} / 2 \mathrm{kT})$ and $\tanh (\mathrm{eV} / 2 \mathrm{kT})$. The former is due to the membrane potential energy barrier and the latter corresponds to saturation effect due to rate limitation of backflow of HA molecules.

For all other types of kinetic limitations

$$
\alpha=E_{i} \cosh (e V / 2 k T) \text {, }
$$

where $E_{i}$ represents the kinetic limitation parameter. The following 
description of the properties of the transient flux obtained by numerical methods is in agreement with the above expressions for $\alpha$.

It was found that under all conditions the relaxation amplitude increased and the relaxation time constant decreased with the increase of voltage across the membrane. These variations are very similar to those shown in Fig. 34 obtained for the dimer model A. When the slow dimerization process was the limiting step, the characteristics of the transient flux were independent of the $\mathrm{pH}$ and the uncoupler concentration. The description of transient flux characteristics for other types of kinetic limitations is given below.

These characteristics were obtained by fitting the current decay at long times, when the current approached steady state, to a single exponential at a voltage of $10 \mathrm{mV}$. In all cases the value of $\mathrm{k}_{0}$, the transmembrane ion translocation rate constant in the absence of a voltage, was set to one; $\gamma$ was also set to one; the equilibrium constant $k_{D} / k_{R}$ of monomers was assumed to be one. The equilibrium constant $\mathrm{k}_{B} / \mathrm{k}_{\alpha}$ of dimers $\mathrm{HA}_{2}^{-}$was set at $10^{3}$ so that the dimer density remained small. The total uncoupler concentration was unity.

Fig. 39 shows the variations of the time constant and relaxation amplitude with $\mathrm{pH}$, under various kinetic limitations. Relaxation amplitude for kinetic limitation due to slow interfacial transfer of anions decreases with the $\mathrm{pH}$ at $\mathrm{pH}>\mathrm{pk}_{\mathrm{a}}$ but attains a steady value at low $\mathrm{pH}$. This results from the fact that the kinetic limitation parameter $E_{4}$ decreases with $\mathrm{pH}$. For other kinetic limitations the relaxation amplitude increases with the $\mathrm{pH}$ for $\mathrm{pH}_{\mathrm{pk}}$ but attains a steady value at high $\mathrm{pH}$. The relaxation time constant increases with the $\mathrm{pH}$ at $\mathrm{pH}>\mathrm{pK}$. 


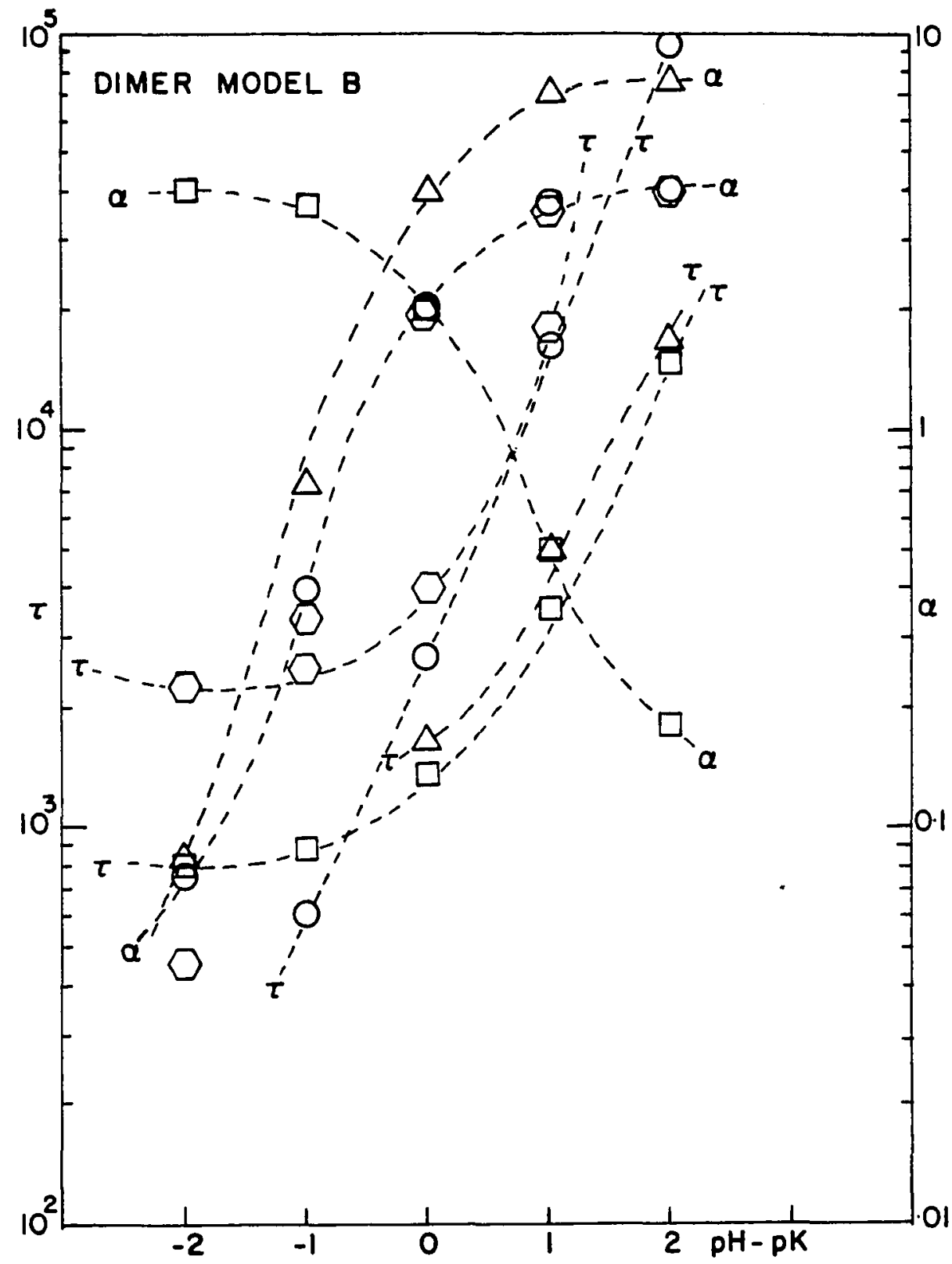

Figure 39. Variation of the time constant $\tau$ and the relaxation amplitude $\alpha$ with the $\mathrm{pH}$ at varioas kinetic limitations; $\Delta$ - slow rate of HA backflow, 0 - slow proton association dissociation reaction, $\square$ - slow interfacial transfer of $\mathrm{A}^{-}, \quad \mathrm{O}$ - slow interfacial transfer of $\mathrm{HA}$. When the rate limiting step is the interfacial transfer of anions $\mathrm{A}^{-}$, the relaxation amplitude decreases with $\mathrm{pH}$ whereas for other kinetic limitations it increases with $\mathrm{pH}$. 
Fig. 40 shows the variations of the time constant and the relaxation amplitude with uncoupler concentration, for various kinetic limitations at $\mathrm{pH}=\mathrm{pK}+1$. The concentration was varied between .25 and 2 . At ali kinetic limitations the relaxation amplitude increased and the time constant decreased with concentration. Fig. 41 illustrates that the relaxation amplitude is proportional to the concentration. 


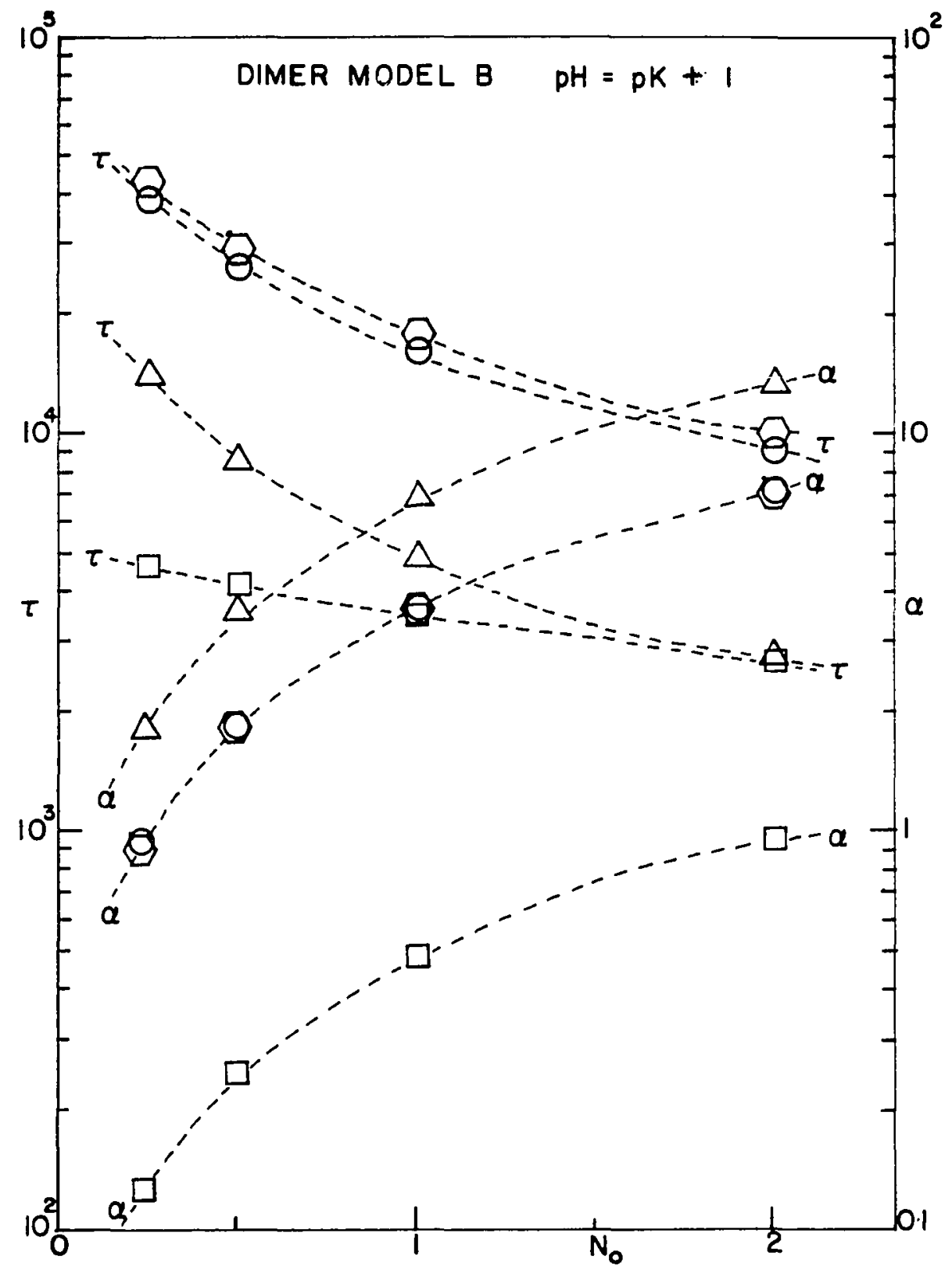

Figure 40. Decrease of the time constant $\tau$ and increase of the relaxation amplitude $\alpha$ with uncoupler concentration $N_{0}$ at various kinetic limitations: $(p H=p k+1) . \quad \Delta$-slow rate of HA backflow, $O$ - slow proton association dissnciation reaction, $\square$ - slow interfacial transfer of $A^{-}, Q$ - slow interfacial transfer of $H A$. 


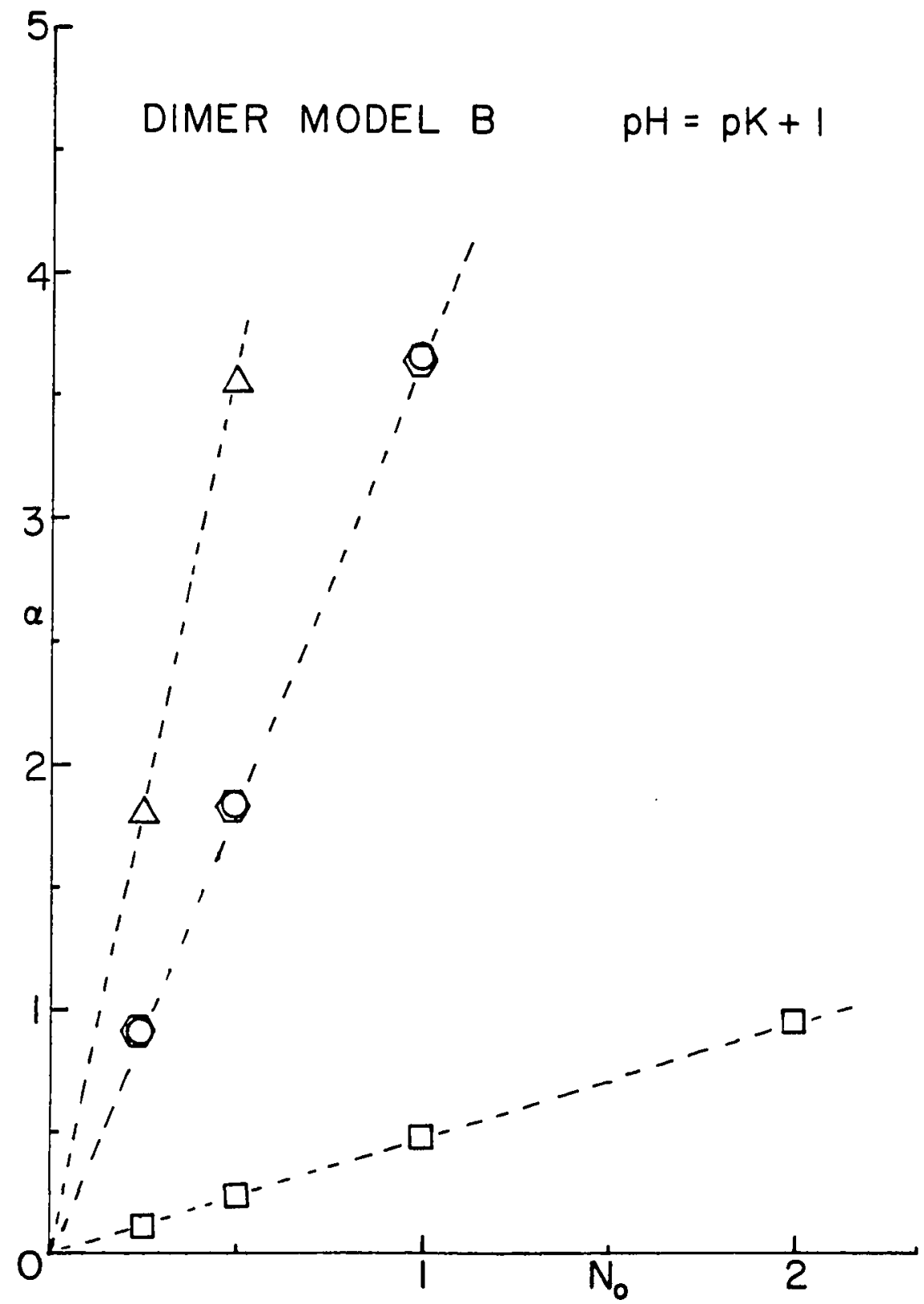

Figure 41. Computed results illustrating that the relaxation amplitude is proportional to the uncoupler concentration $\mathrm{N}_{0}$ (Eqs. 322, 323); $(\mathrm{pH}=\mathrm{pK}+1) . \Delta$ - slow rate of HA backflow, O - slow proton association dissociation reaction, $\square$ slow interfacial transfer of $\mathrm{A}^{-}, \quad \mathrm{O}$ - slow interfacial transfer of HA. 
CHAPTER IV

RESULTS

PH DEPENDENCE OF MEMBRANE CONDUCTIVITY

Fig. 42 shows the $\mathrm{pH}$ dependence of the steady state membrane conductance induced by PCBT incorporated into the membrane phase. The solid line is a plot of $h /(1+h)^{2}$, where $h$ is the reduced proton activity given by $h=a_{H} /\left(k_{R} / k_{D}\right)$. The curve is adjusted so as to go through the mean of the experimental data points. The points indicated by squares represent the $\mathrm{pH}$ dependence of the uncoupling activity of PCBT in rat liver mitochondria, as reported by wilson et. al. (34). Data representing uncoupling activity are reproduced from Fig. 5 of ref. (34), and are equal to the slopes of the titration curves of rat Iiver mitochondria with PCBT at the designated $\mathrm{pH}$ values.

There are several important conclusions that can be drawn from Fig. 42. These are:

(1) The correlation between the membrane conductivity and the uncoupling activity induced by PCBT is consistent with the hypothesis that the mechanism of uncoupling is the same as the mechanism by which weak acids induce conductivities in lipid bilayers ( 8 ).

(2) The fit of the experimental conductivities to the curve $h /(1+h)^{2}$ is consistent with the dimer hypothesis which postulates that the membrane permeable charged species in membrane transport due to weak acids is a dimer $\mathrm{HA}_{2}^{-}$formed by the combination of $\mathrm{HA}$ and $A^{-}$ species $(44,45)$. 


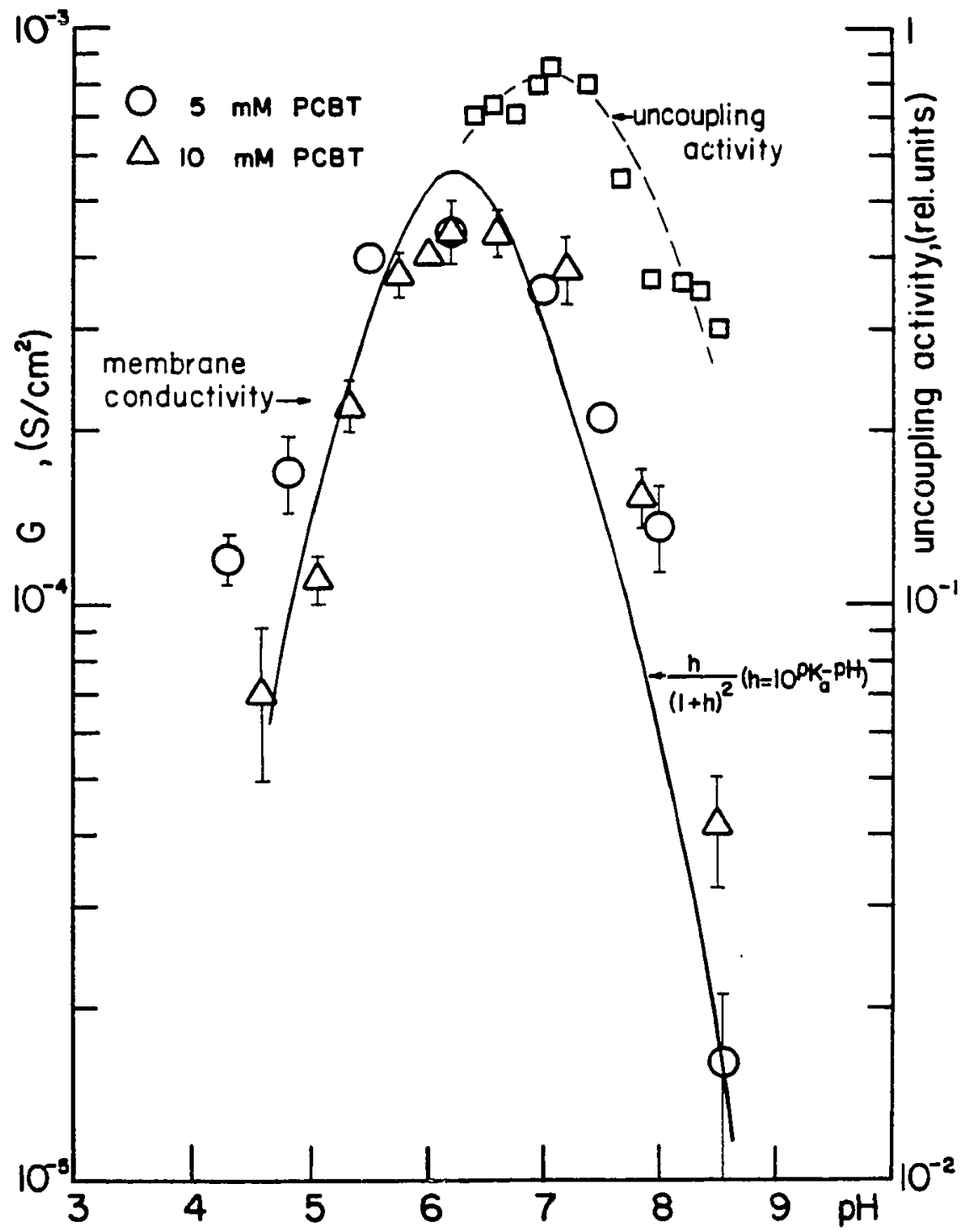

Figure 42. $\mathrm{pH}$ dependence of the membrane conductivity and $\mathrm{pH}$ dependence of the uncoupling activity induced by PCBT. The similarity of curves indicates that the action of PCBT in biomembranes and artificial membranes are the same. Fit of the conductivity to the solid curve supports the dimer hypothesis. 
Fig. 43 shows the $\mathrm{pH}$ dependence of the membrane conductivity induced by PCP incorporated into the membrane phase. The fit of the conductivity data to the solid curve, which represents $h /(1+h)^{2}$ is consistent with the dimer hypothesis mentioned above. The dashed Iines drawn through the points represented by triangles indicate the variation of the membrane bound negative charge density with pH obtained from the microelectrophoresis experiments. Since the variation of membrane surface charge density (dashed curve) with the pH is similar to the variation of the anion $\mathrm{A}^{-}$density with $\mathrm{pH}$

$$
\mathrm{n}_{\mathrm{A}}^{\text {in }}=\frac{\mathrm{N}_{\mathrm{O}} / 2}{1+10^{\mathrm{pK}}-\mathrm{pH}}
$$

but entirely different from the variation of the dimer $\mathrm{HA}_{2}^{-}$density with $\mathrm{pH}$

$$
\mathrm{n}_{\mathrm{HA}_{2}}=\frac{\mathrm{k}_{\alpha}}{\mathrm{K}_{\mathrm{B}}} \frac{\left(\mathrm{N}_{0} / 2\right)^{2} 10^{\mathrm{pk}_{\mathrm{a}}-\mathrm{pH}}}{\left(1+10^{\mathrm{pk}} \mathrm{a}^{-\mathrm{pH}}\right)^{2}}
$$

the result indicates that the dimer density in the membrane is small compared to the anion density.

Fig. 44 shows the variation of the membrane conductivity with the $\mathrm{pH}$ at three levels of concentration of $2,4,5-\mathrm{TCP}$ in the aqueous phase. Only the conductivities corresponding to the lowest concentration of $2,4,5-T C P$ fitted well the curve $h /(1+h)^{2}$, represented by the solid line. At high concentrations of 2,4,5-TCP the $\mathrm{pH}$ of the conductivity maximum is displaced to a high $\mathrm{pH}$. In addition there appears to be asymmetry of the conductance against $\mathrm{pH}$ data about the $\mathrm{pH}$ of the conductance maximum; there is a rapid decrease of the conductance with pH when $2,4,5-\mathrm{TCP}$ becomes fully ionized. 


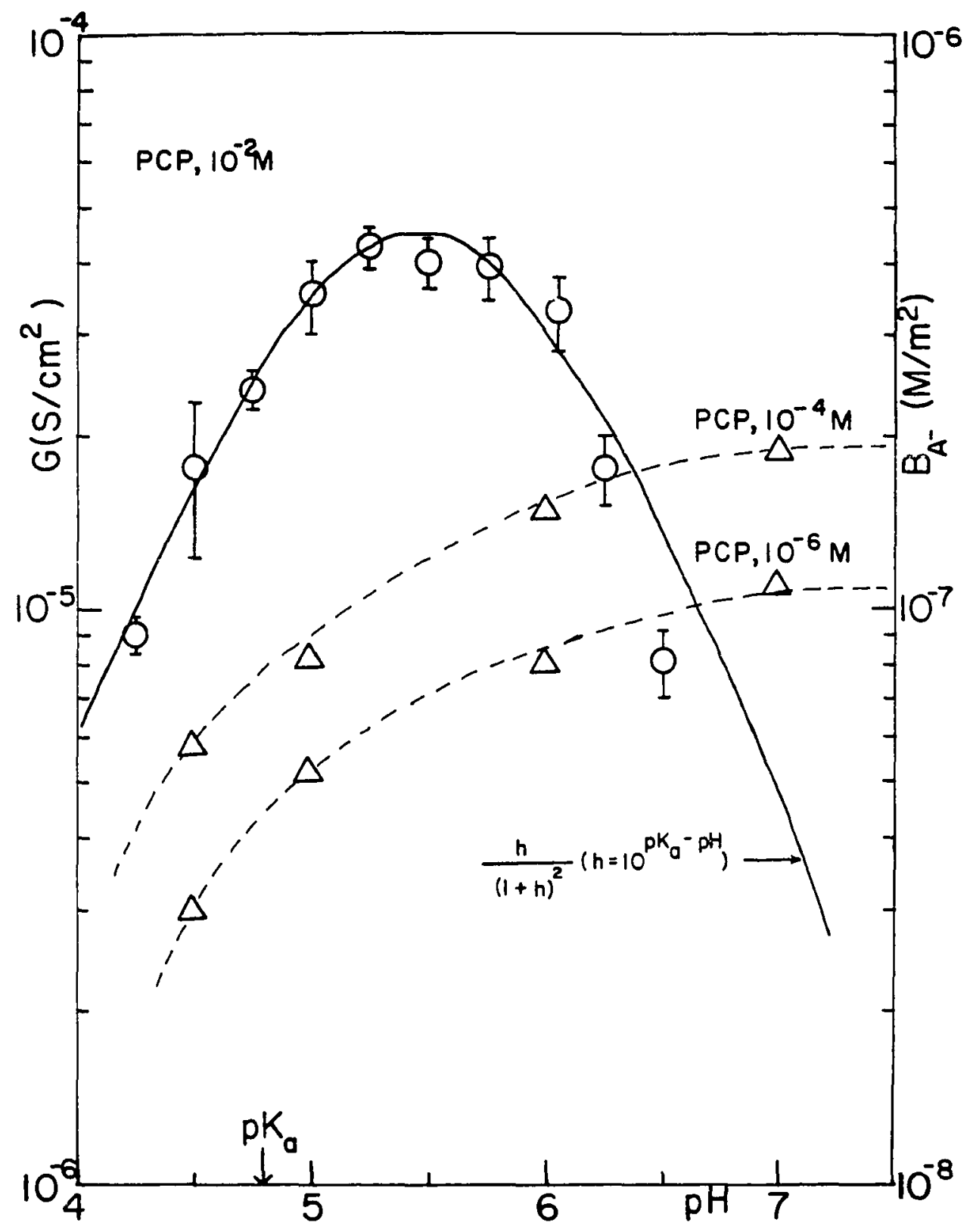

Figure 43. $\mathrm{pH}$ dependence of the PCP induced membrane conductivity (circles), and $\mathrm{pH}$ dependence of the density of PCP ions adsorbed to vesicles (triangles). Fit of the membrane conductivity to the solid curve supports the dimer hypothesis. Variation of the bound PCP ion density (dashed curve) is similar to the variation of PCP anion concentration with $\mathrm{pH}$ and therefore indicates that the dimer density is small compared to that of anions, at the membrane surface. 


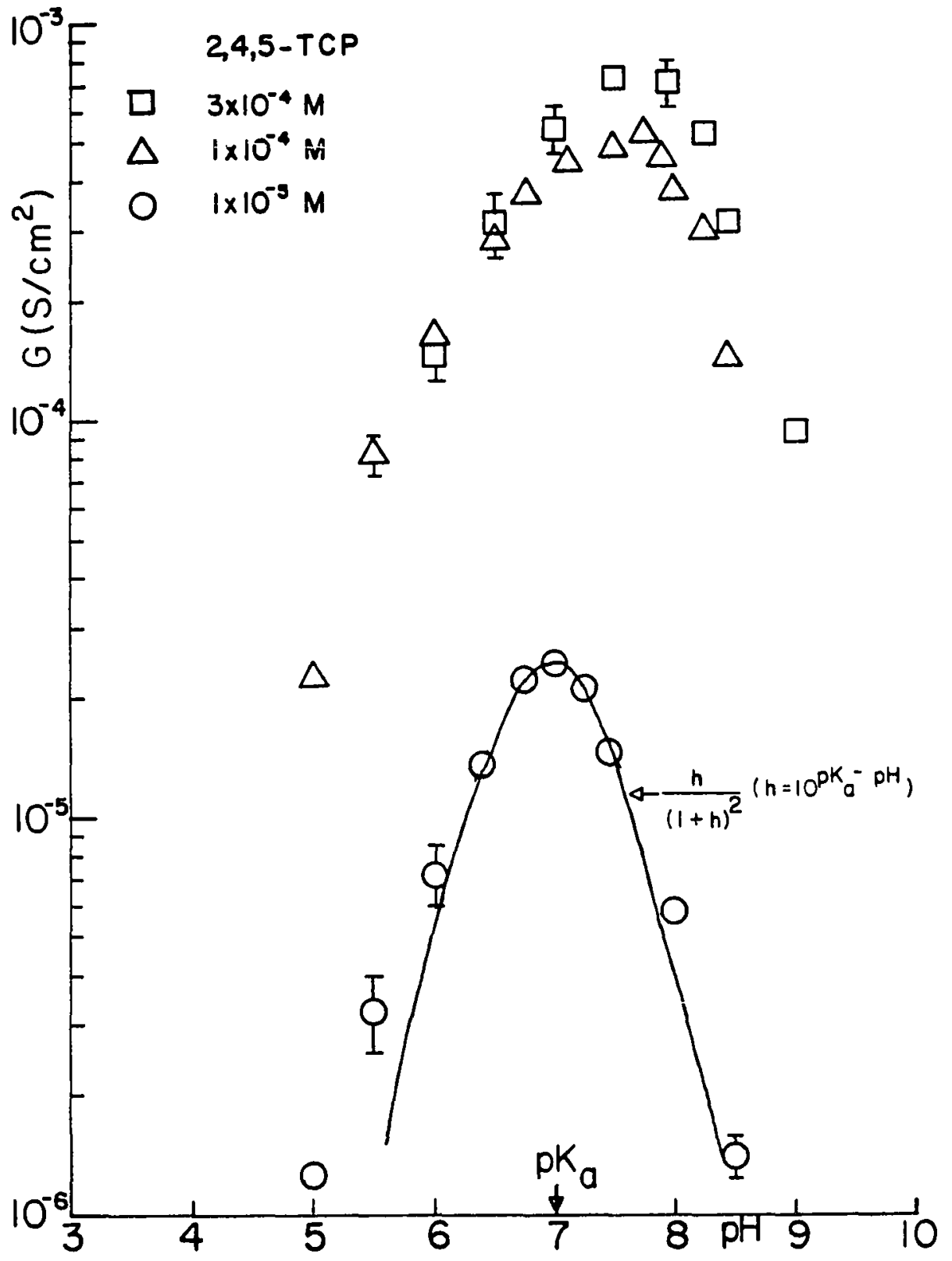

Figure 44. $\mathrm{pH}$ dependence of the 2,4,5-TCP induced membrane conductivity. Fit of the conductivity to the solid curve at the lowest concentration supports the dimer hypothesis. 
It is important to note by comparing Figs. 42 and 43 , that PCP induced conductivities are about one order of magnitude lower than those induced by its sulfur analog, PCBT, at the same concentration.

\title{
CONCENTRATION DEPENDENCE OF MEMBRANE CONDUCTIVITY
}

\author{
Fig. 45 shows the variation of the steady state membrane \\ conductance with the concentration of PCBT in the membrane. The dashed \\ Iines correspond to a slope of two. The data suggest that the experi- \\ mental membrane conductance is proportional to the square of the uncoup- \\ ler concentration. The results are in agreement with the dimer hypoth- \\ esis, according to which the charge transporting species through the \\ membrane is the dimer $\mathrm{HA}_{2}^{-}$formed by the combination of $\mathrm{HA}$ and \\ $A^{-}(44,45)$ \\ As shown in Fig. 46 the dependence of conductance on the concen- \\ tration of PCP in membrane phase is less than quadratic. The less than \\ quadratic dependence of conductance on concentration has been observed \\ earliex for FCP (28), and another uncoupler DNP (7). This behavior has \\ been shown to be due to the negative surface charge caused by the \\ adsorption of uncoupler anions $(7,28)$. \\ Fig. 47 shows the dependence of membrane conductance as a \\ function of the aqueous concentration of 2,4,5-TCP. Except at very high \\ $\mathrm{pH}$ the membrane conductance is proportional to the square of the \\ $2,4,5-T C P$ concentration as indicated by the fit of the data to the \\ dashed line of slope two. At very high concentrations the curves have a \\ very interesting feature not seen before for any other uncoupler; \\ membrane conductance goes through a maximum; and any further increase \\ of 2,4,5-TCP concentration results in a decrease of conductivity. The
}




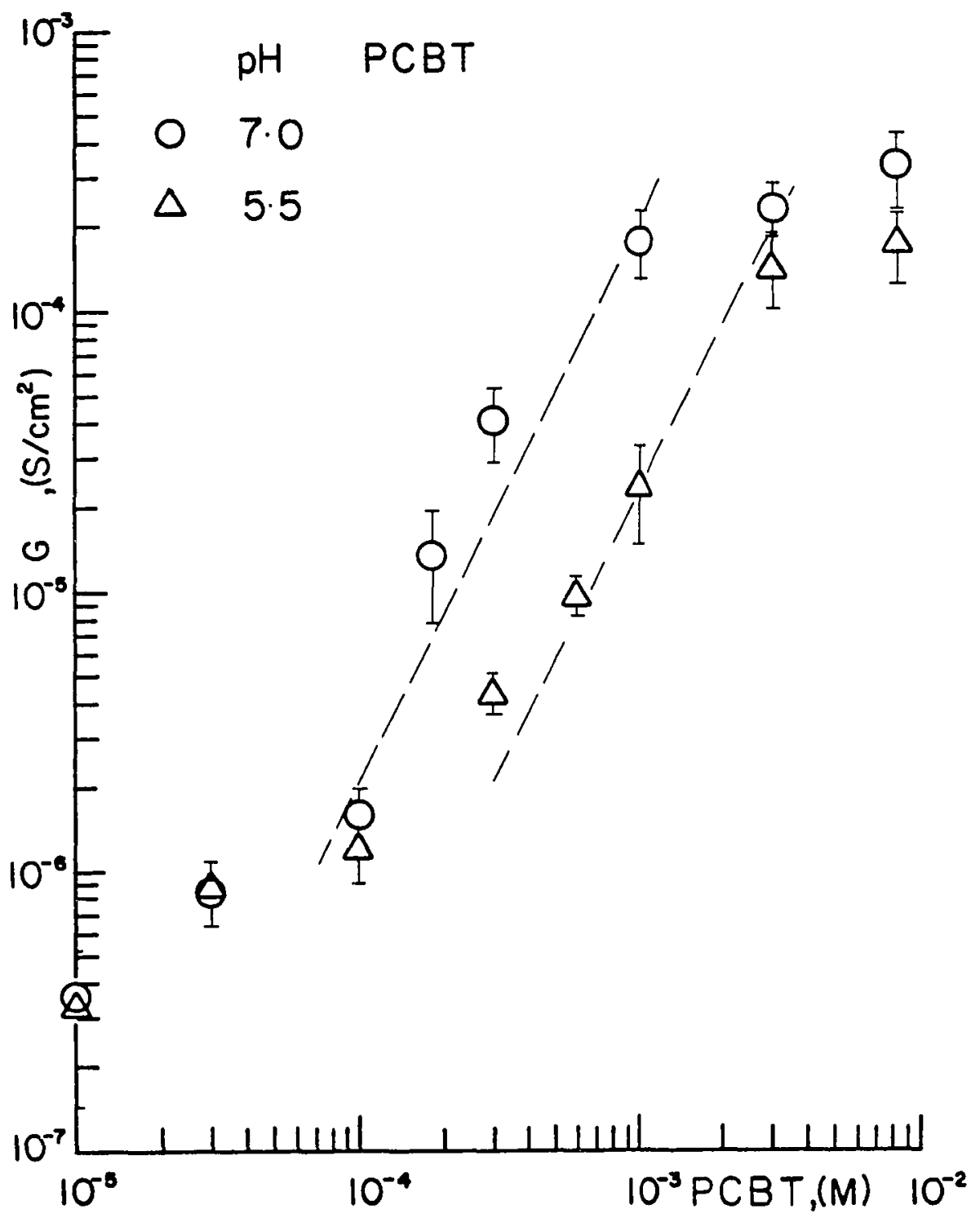

Figure 45. Dependence of the membrane conductance on the PCBT concentration. The broken lines indicate a quadratic dependence of conductance on concentration, and it is in agreement with the dimer hypothesis. 


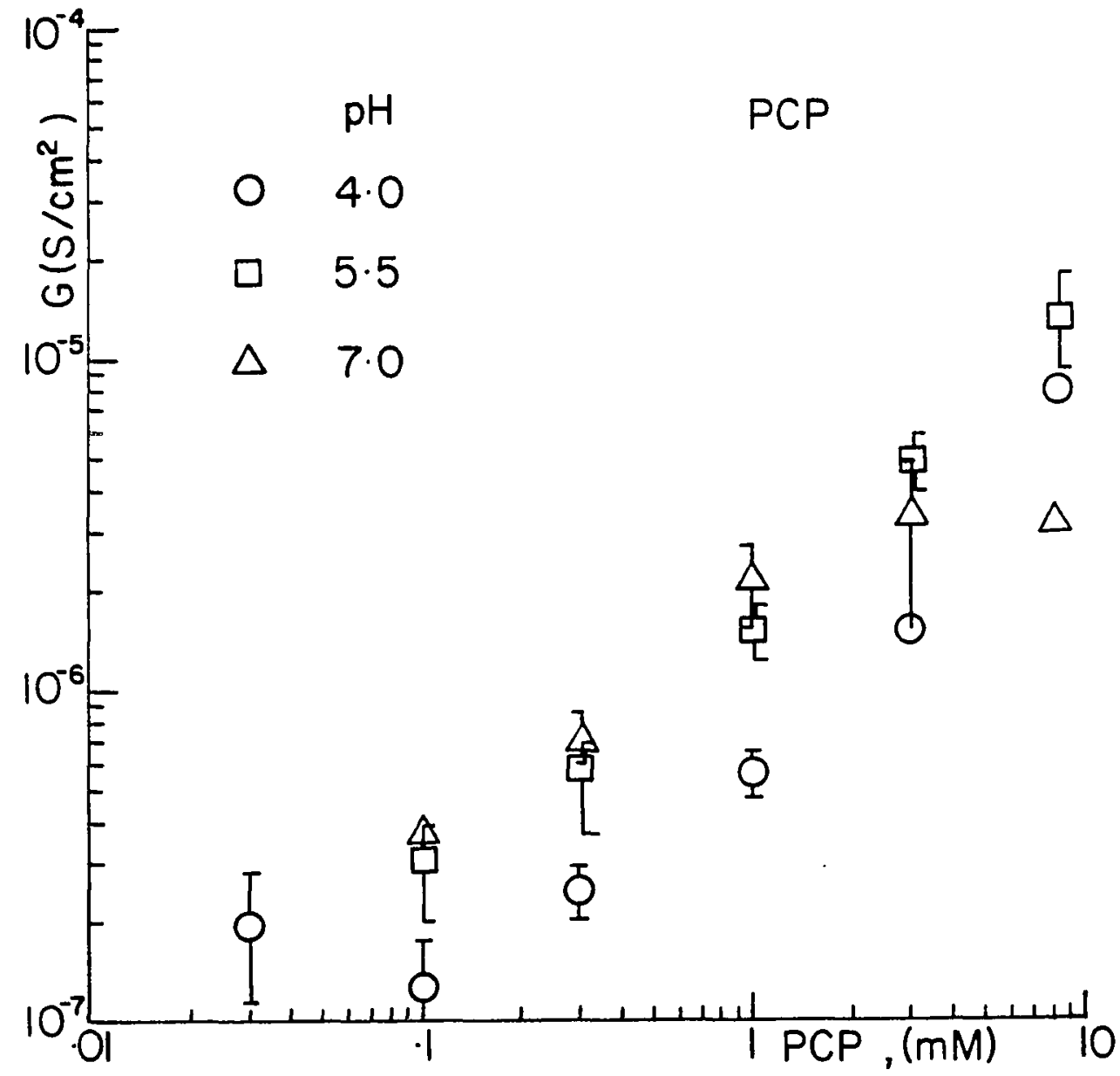

Figure 46. Dependence of the membrane conductance on the PCP concentration. The less than quadratic dependence of conductance on PCP concentration has been shown to be due to the negative surface charge caused by the adsorption of uncoupler anions. 


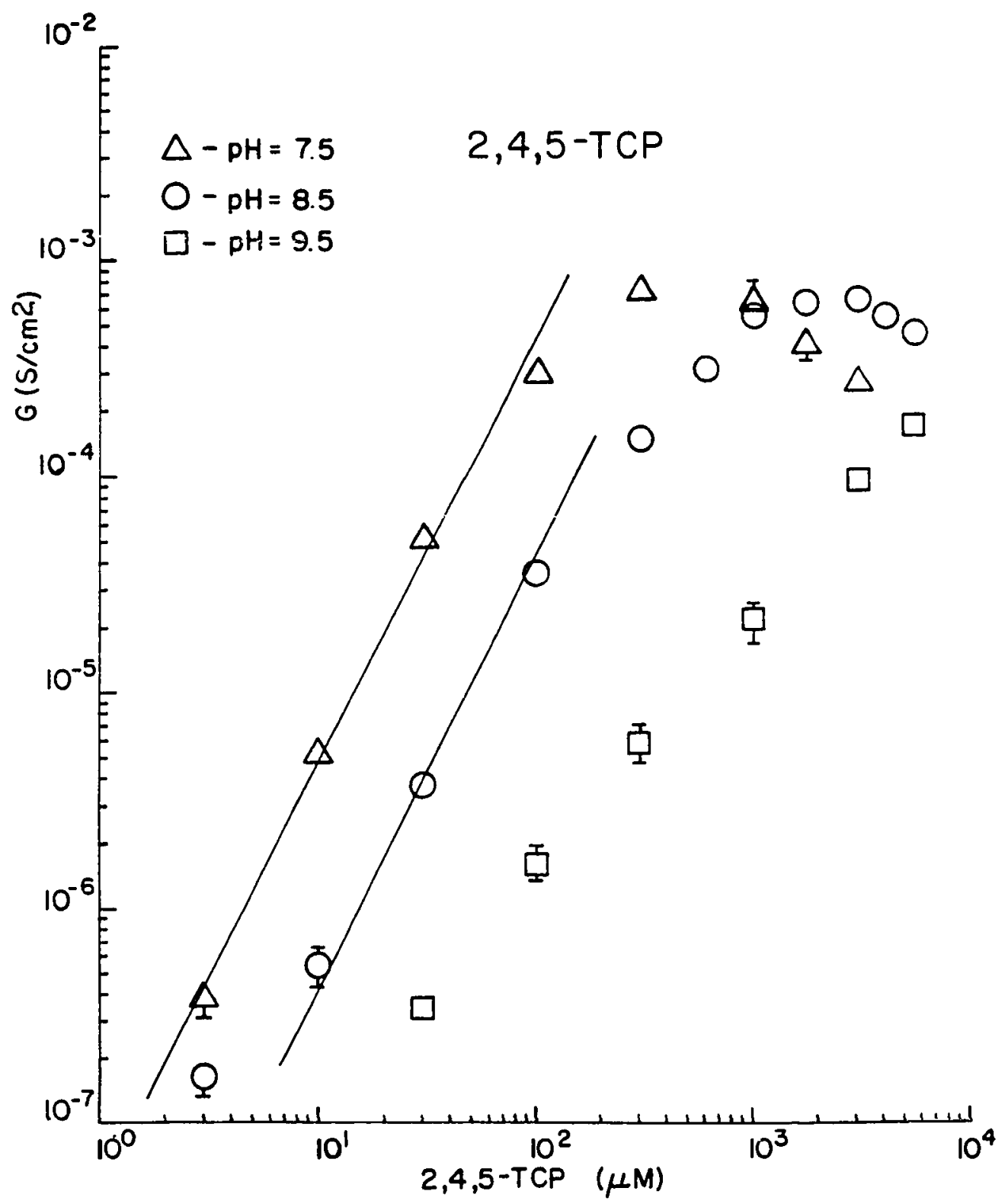

Figure 47. Dependence of the membrane conductance on 2,4,5TCP concentration. The broken lines indicate a quadratic dependence of conductance on the concentration. The fit of the points to the line is consistent with the dimer hypothesis. 
quadratic dependence of conductivity on uncoupler concentration is consistent with the dimer hypothesis mentioned before. The less than quadratic dependence of conductance on concentration at pH of 9.5 may be related to the effect of adsorbed anions, similar to that observed for PCP.

MEMBRANE POTENTIAL

Fig. 48 shows the variation of membrane potential per unit of transmembrane $\mathrm{pH}$ difference as a function of the $\mathrm{pH}$ of the aqueous phase. The membrane potential of the low $\mathrm{pH}$ side was found to be negative with respect to the high $\mathrm{pH}$ side. The value of about $51 . \mathrm{mV}$ observed approximately corresponds to the Nernst potential for a decade of hydrogen ion activity difference (29). However, as pointed out in Chapter $V$, the occurrence of membrane potential difference corresponding to the Nernst value for the transmembrane proton gradient does not necessarily indicate that the membrane is permeable to $\mathrm{H}^{+}$or $\mathrm{OH}^{-}$ions.

\section{EFFECT OF LOW BUFFER CAPACITY}

Fig. 49 shows the zero voltage conductance, obtained by extrapolating the conductance to zero voltage, at three $\mathrm{pH}$ values and for low and high buffer concentrations. At low buffer concentration the conductivities were reduced by about a factor of ten. The bell shaped appearance of the $\mathrm{pH}$ dependence of the membrane conductivity, observed at high buffer concentration, disappears at low buffer concentration. The above observations indicate that both the protons and buffer ions are necessary for maintaining the continuity of ion flux across the aqueous unstirred layers $(8,28,30)$. At low buffer concentration, the 


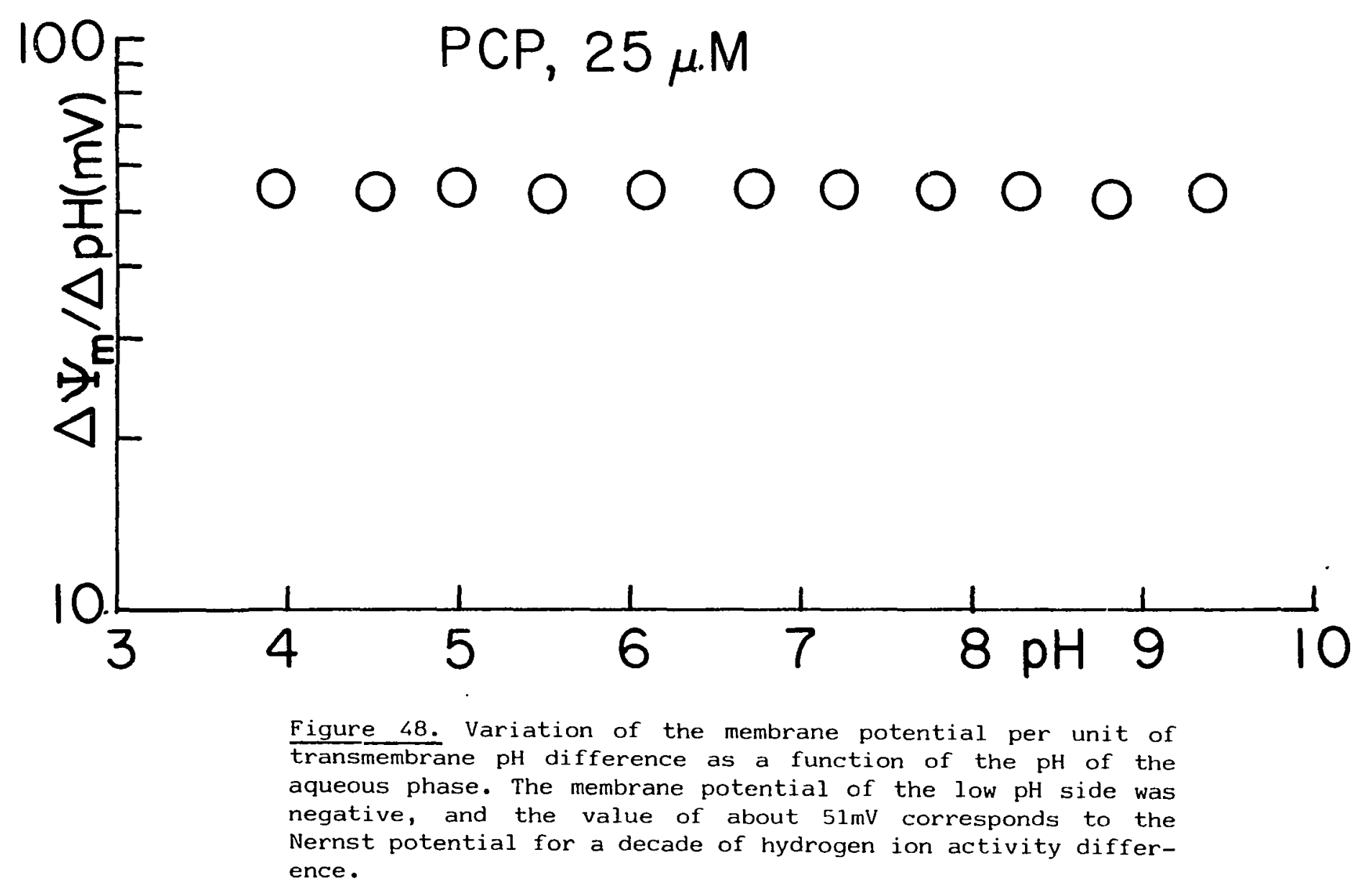




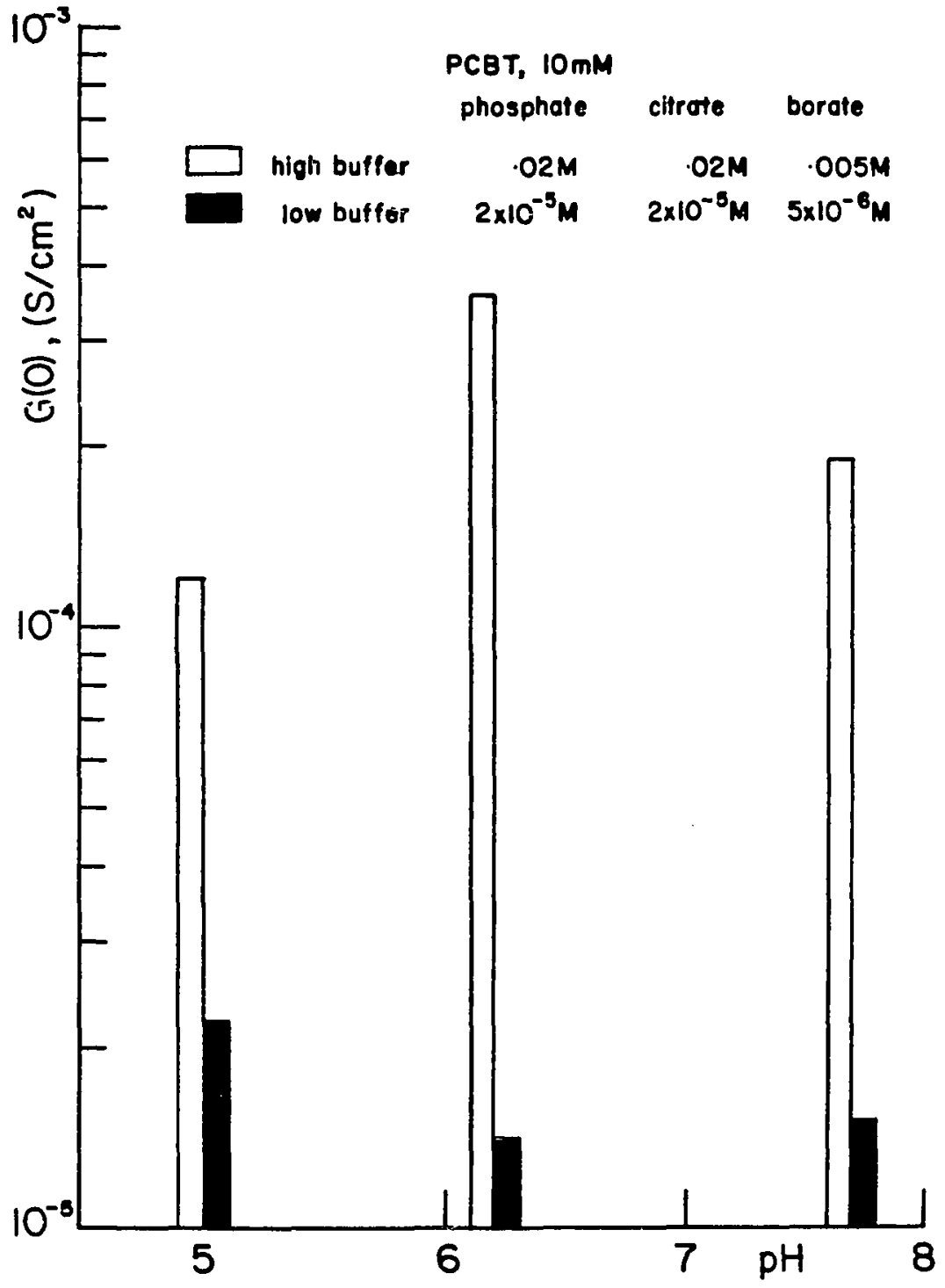

Figure 49. Effect of the buffer concentration on PCBT induced membrane conductance. At low buffer concentration the conductivity is reduced and the bell shaped pH dependence disappears indicating that protons and buffer ions carry the ion flux across the aqueous unstirred layers. 
ion flux through the membrane is limited by the diffusion of protons in the aqueous unstirred layers. This has been verified by measurement of slow transient currents.

At low buffer concentration a PCBT induced slow current transient was observed when a voltage step was applied across the membrane. Fig. 50 indicates that the transient current is proportional to the reciprocal of the square root of time. This result is in agreement with the theory of diffusion polarization applied to membrane transport by Neumcke (47), who showed that such a dependence of current on time (Eq. 331) exists for transmembrane flux limited by the diffusion in the aqueous unstirred layers adjacent to the membrane.

\section{NORMALIZED MEMBRANE CONDUCTIVITY}

\section{Absence of Kinetic Limitations}

According to the dimer models of ion transport in membranes (Chapter III), the variation of the normalized membrane conductivity with voltage, in the absence of kinetic limitations, is given by (Eqs. $237,312)$

$$
\frac{G(V)}{G(0)}=\frac{\sinh (\mathrm{eV} / 2 \mathrm{kT})}{(\mathrm{eV} / 2 \mathrm{kT})}
$$

In Eq. 326 we introduced an empirical coefficient $B$, which represents the fraction of the applied voltage displacing the dimers inside the membrane. This was found recessary in order to fit the experimental results to the simple model of membrane ion transport.

Fig. 51 shows the variation of the normalized membrane conductivity with voltage at low concentration of the uncoupler $2,4,5-$ TCP. 


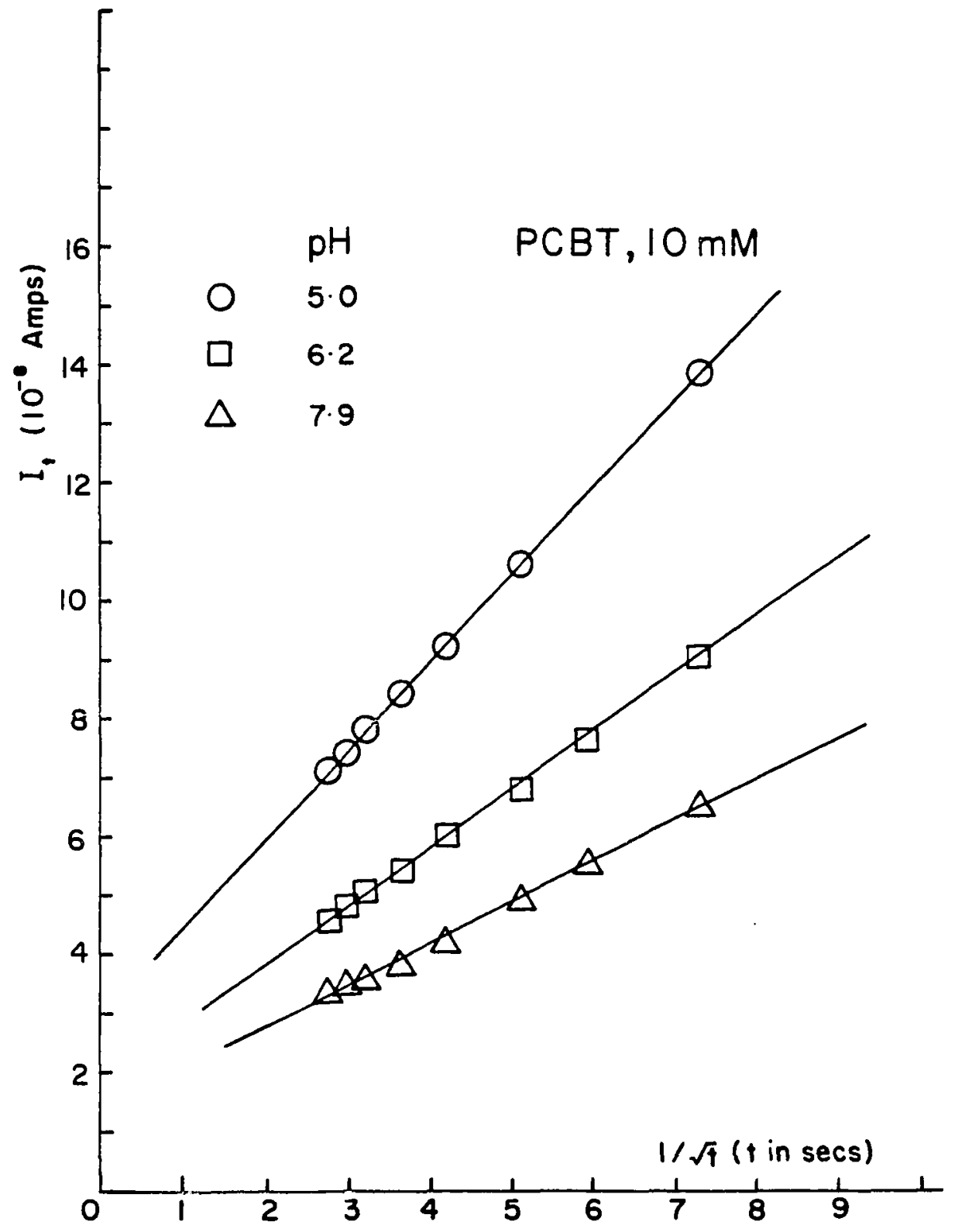

Figure 50. Dependence of the PCBT induced slow transient current observed at low aqueous buffer concentration (phosphate:citrate; borate $\left.=2 \times 10^{-5} \mathrm{M}: 2 \times 10^{-5} \mathrm{M}: 5 \times 10^{-6} \mathrm{M}\right)$ on the reciprocal of the square root of time. Fit of the points to a straight line agrees with Eq. 331 derived by Neumcke (47), and shows that the transmembrane flux is limited by diffusion of ions across the aqueous unstirred layers. 


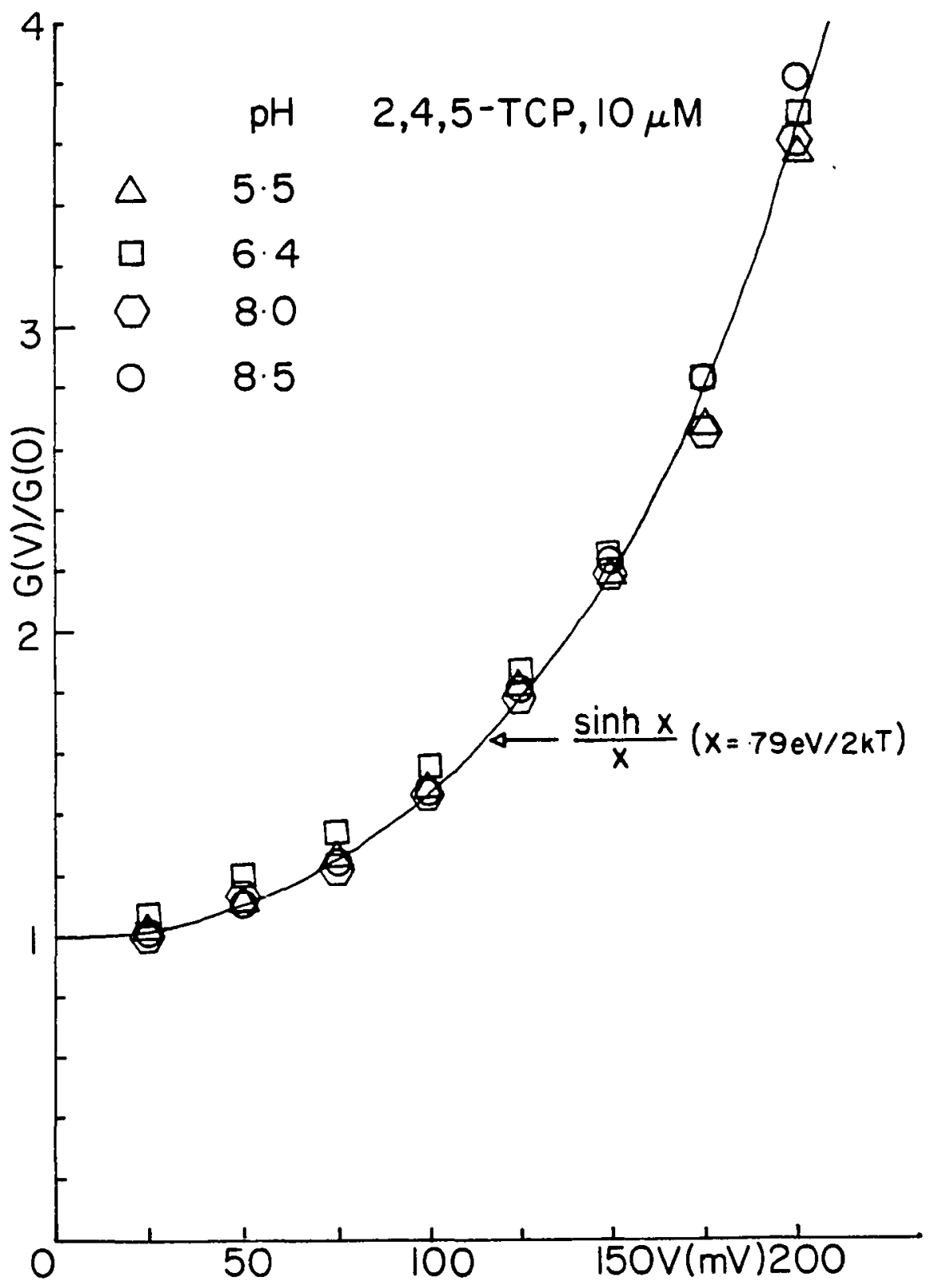

Figure 51. Variation of the 2,4,5-TCP induced normalized membrane conductance with the voltage when there are no kinetic limitations. Best fit of the experimental data to the equation $\sinh (x) /(x) \quad(x=B e V / 2 k T)$ yielded the value for $B=$ $0.79 \pm .03$. 
There are no indications of the presence of kinetic limitations at this low concentration of 2,4,5-TCP. The solid curve represents the function $\sinh (B e V / 2 k T) /(B e V / 2 k T)$ for $B=0.79$, obtained as the average of the least square fit values of $\beta$ from nine sets of data, corresponding to nine different $\mathrm{pH}$ conditions.

Fig. 52 shows the variation of the normalized membrane conductivity with voltage for PCP, at different values of $\mathrm{pH}$. The solid curve represents the function $\sinh (\beta \mathrm{eV} / 2 \mathrm{kT}) /(\mathrm{BeV} / 2 \mathrm{kT})$ for $\beta=0.78$ obtained as the average of the least square fit values of $B$ from nine sets of data, at nine different $\mathrm{pH}$ conditions. The value of $B$ obtained for PCP is about the same as that for 2,4,5-TCP.

Since strong kinetic limitations were observed under most conditions for PCBT treated membranes, we used the initial current obtained from current relaxation experiments to determine B. Dimer models (Chapter III) predict that

$$
\frac{G(0, V)}{G(0,0)}=\frac{\sinh (B e V / 2 k T)}{(B e V / 2 k T)}
$$

where $G(O, V)$ is the initial conductance obtained by extrapolating the current transient under a voltage step $V$ to zero time. $G(0,0)$ is obtained by extrapolating the initial conductance $G(0, V)$ to zero voltage. Fig. 53 shows the variation of $G(0, V) / G(0,0)$ with voltage. Solid curve is the least squares fit of the function given in Eq. 327 to the data. The best value of $B$ obtained is 0.7 . The values of $\beta$ obtained for PCBT, PCP, and $2,4,5,-$ TCP agree with each other and with the values reported for other membrane modifiers $(38,58)$ indicating that only 70 to $80 \%$ of the applied voltage drives the membrane permeable ion across the membrane. 


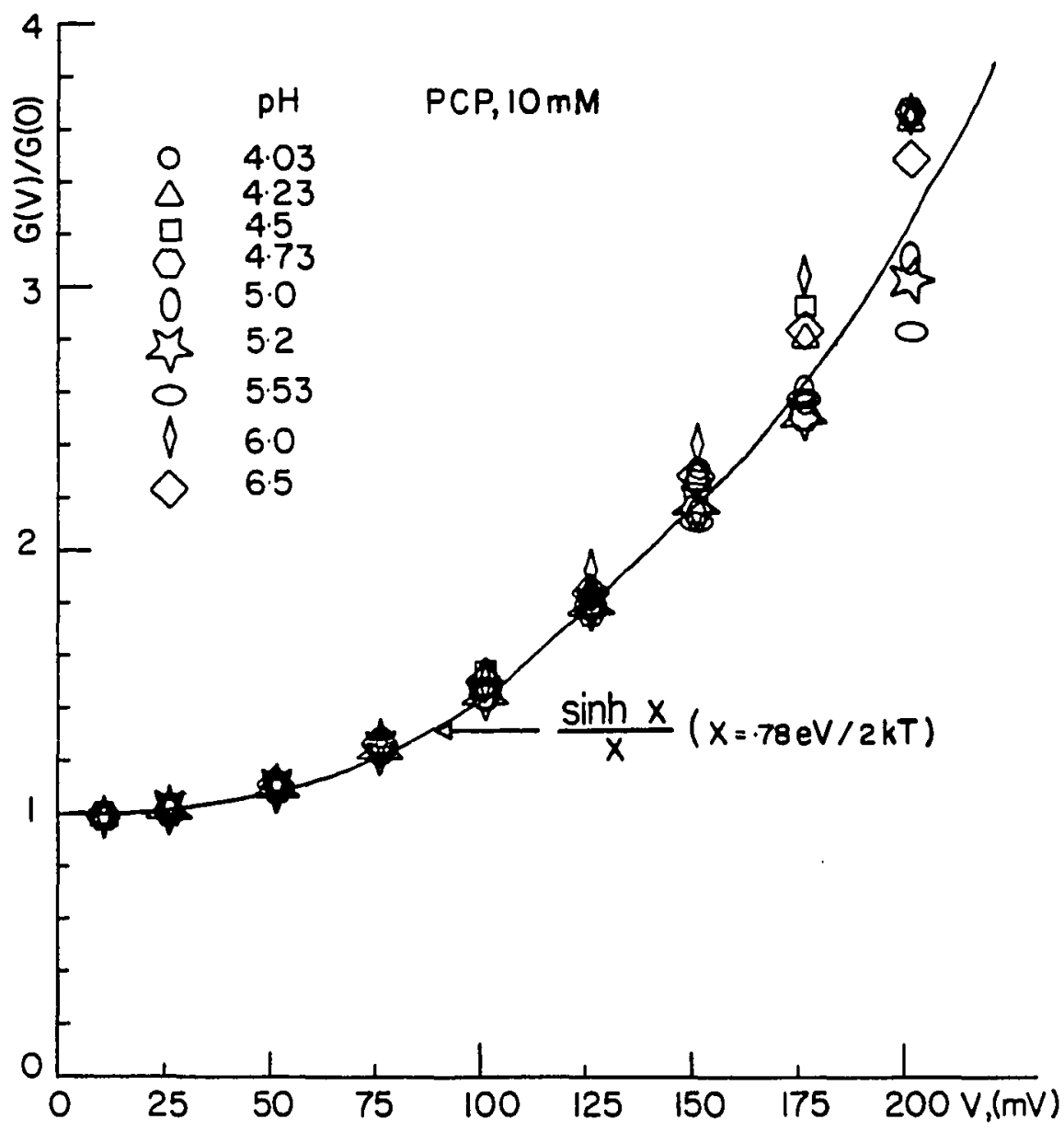

Figure 52. Variation of the PCP induced normalized membrane conductance with voltage. Best fit of the experimental data to the equation $\sinh (x) /(x) \quad(x=B e V / 2 k T)$ yielded the value for $B=0.78 \pm .04$. 


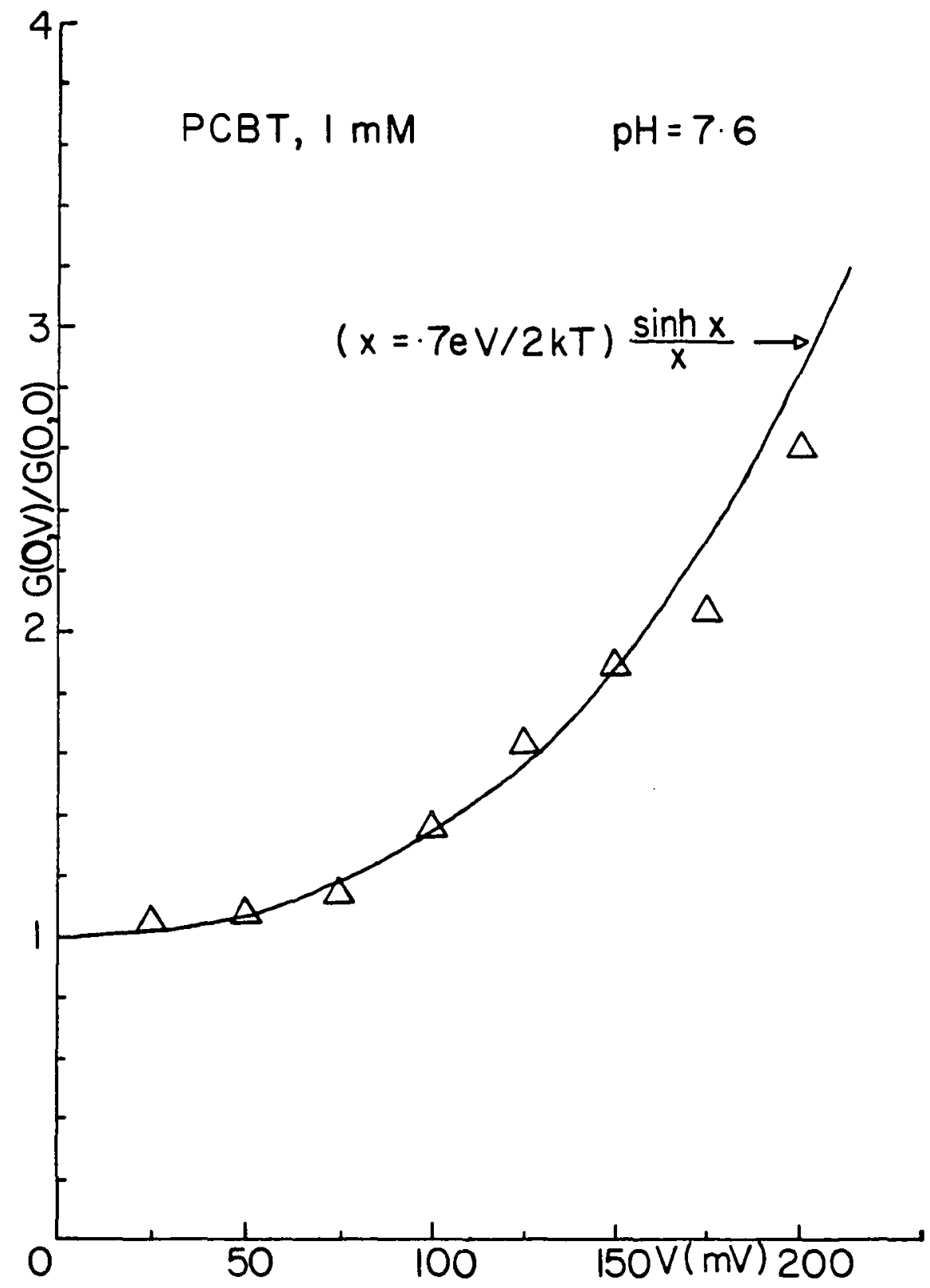

Figure 53. Variation of the PCBT induced normalized initial membrane conductance, obtained from current relaxation data, with the voltage. Best fit of the points to the Eq. 327 yielded the value for $\beta=0.7$. 


\section{Presence of Kinetic Limitations}

Under most experimental conditions the normalized membrane conductances observed for PCP were similar to those shown in Fig. 52, which indicates that for PCP the kinetic limitations of membrane conductance are small. For 2,4,5-TCP the existence of kinetic limitations was observable only at the highest concentration. Fig. 54 shows the variation of normalized membrane conductance with voltage for $3 \times 10^{-4} \mathrm{M}$ $2,4,5-\mathrm{TCP}$. The conductance becomes less voltage dependent as the $\mathrm{pH}$ is increased, indicating that the kinetic limitations increase with the $\mathrm{pH}$. The solid curves represent the function

$$
\frac{G(V)}{G(O)}=\frac{\sinh (B e V / 2 k T)}{(B e V / 2 k T)} \frac{1+E_{O}}{1+E_{0} \cdot \cosh (B e V / 2 k T)}
$$

(Eq. 237) for $B=0.79$ and for various values of $E_{0}$. The dimer models predict that the normalized membrane conductance varies according to Eq. 328. The kinetic Iimitations are measured by the magnitude of the net kinetic limitation parameter $E_{0}$.

As shown in Fig. 55, the normalized conductance of PCBT treated membranes under most conditions decreases with the applied voltage, indicating existence of strong kinetic limitations. The solid curves in Fig. 55 represent the function 328 for $\beta=0.7$, and for various values of $E_{O}$. Another important point to notice in Fig. 55 is that the conductance decreases with voltage more rapidly as the pH was either increased or decreased from the value of the $\mathrm{pH}$ corresponding to maximum conductivity $(\mathrm{pH}=6.6$, Fig. 42). Data in Fig. 55 indicate the existence of kinetic limitations for PCBT induced conductivity at any $\mathrm{pH}$ above or below the $\mathrm{pH}$ at which conductance is maximum (refer Fig. 42) 


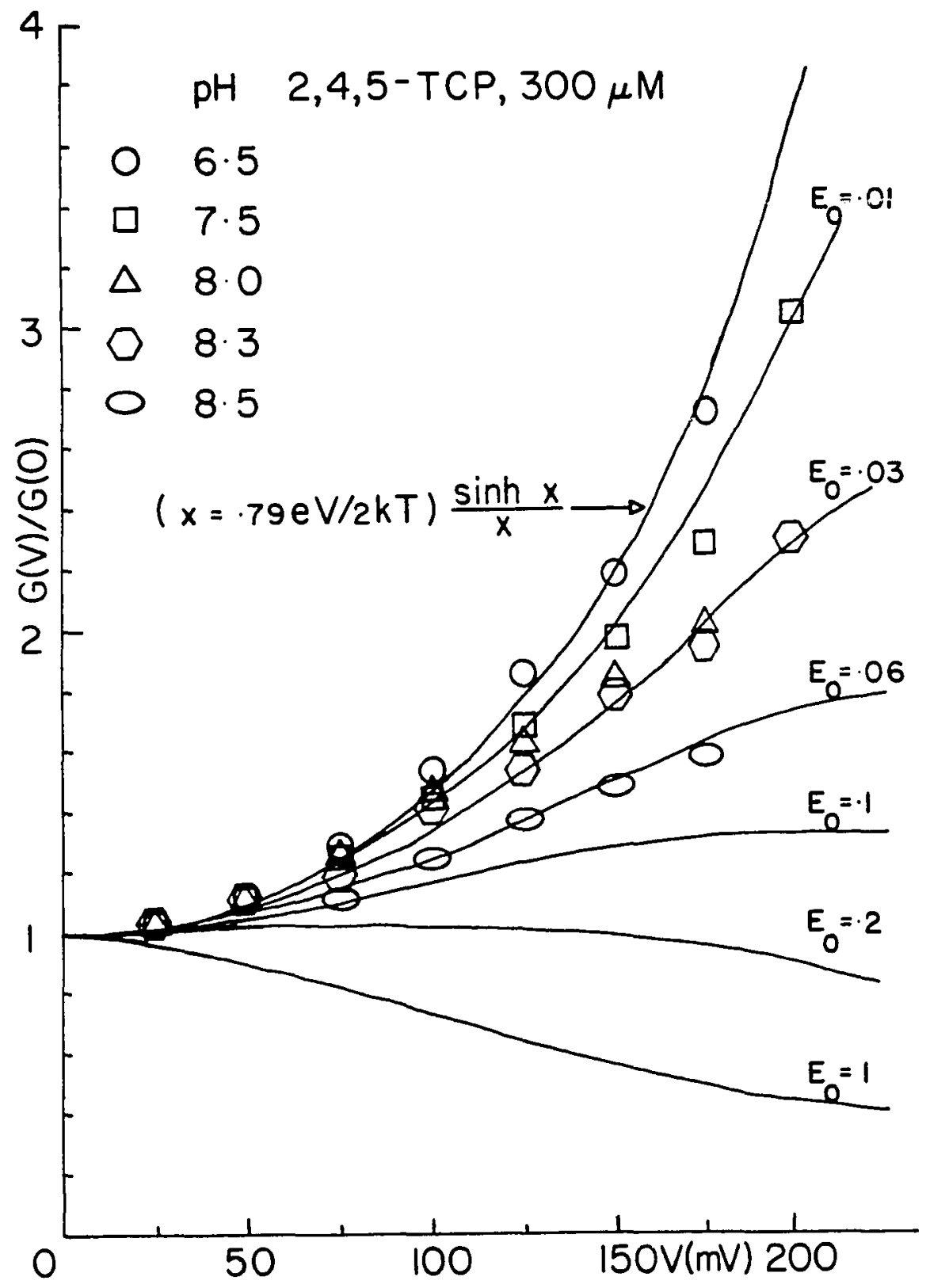

Figure 54. Voltage dependence of the 2,4,5-TCP induced normalized membrane conductance at high concentration of the uncoupler, indicating the development of kinetic limitations at high $\mathrm{pH}$. The solid lines represent the Eq. 328 for various values of the kinetic limitation parameter $E_{0}$ and for $B=$ 0.79 . 


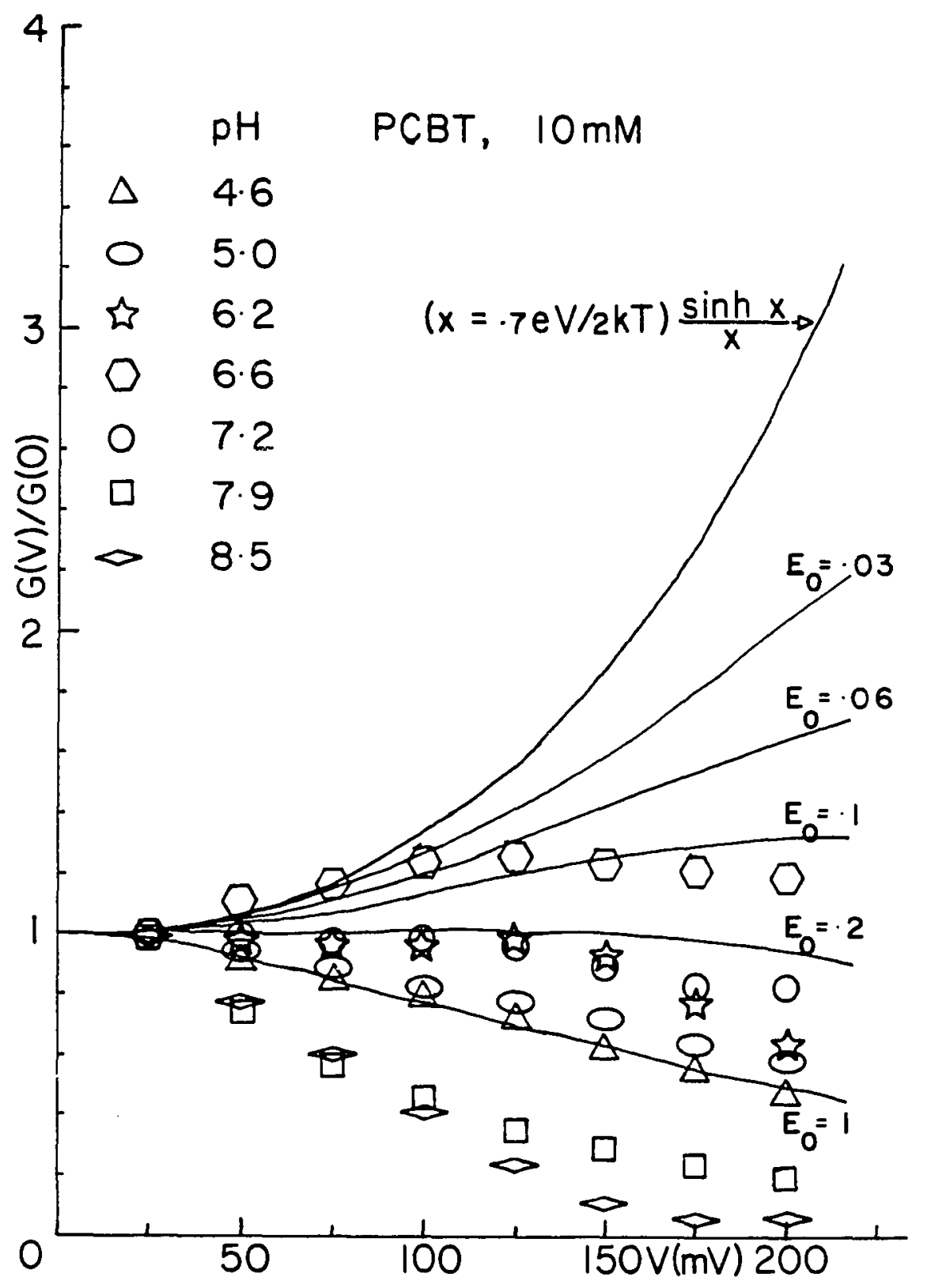

Figure 55. Voltage dependence of the PCBT induced normalized membrane conductance, indicating the development of kinetic limitations at both above and below the $\mathrm{pH}$ corresponding to maximum conductivity. The solid lines represent the Eq. 328 for various values of the kinetic limitation parameter $E_{0}$ and for $\beta=0.7$. 
Fig. 56 is a plot of the PCBT induced normalized membrane conductance as a function of the applied voltage for increasing concentrations of PCBT. The data indicate that with increasing PCBT concentration the kinetic limitations become more prominent. Dimer models (Chapter III) predict that the kinetic limitation parameter is proportional to the concentration of the uncoupler. Therefore, the experimental results in Fig. 56 are in agreement with the predictions of dimer models. However, it is appropriate to point out one inconsistency in the presented results; the points corresponding to the lowest concentration of PCBT lie above the function $\sinh (B \mathrm{eV} / 2 \mathrm{kT}) /(\mathrm{BeV} / 2 \mathrm{kT})$, for $B=$ 0.7. This is very likely due to the fact that the membrane current at this low concentration of membrane modifier was in part due to membrane electrical leakage whose mechanism is different from that induced by PCBT.

\section{ADSORPTION OF PENTACHLOROPHENOL ANIONS TO MEMBRANES}

Fig. 57 shows the variation of the electrophoretic mobility (and the corresponding electric potential at the hydrodynamic plane of shear) of phosphatidylcholine vesicles as a function of PCP concentration, for different values of $\mathrm{pH}$. The density of PCP anions adsorbed to vesicles is calculated from the results of Fig. 57 using diffuse double layer theory (59). The variation of the adsorbed PCP anion density $B_{A}-$ with the aqueous $\mathrm{PCP}$ anion concentration $\mathrm{F}_{\mathrm{A}^{-}}$, for various values of $\mathrm{pH}$, is shown in Fig. 58 .

There are two sources of error in the aqueous concentration of PCP anions, of which one has been corrected and the other has not. The decrease of aqueous PCP anions due to the adsorption of anions to 


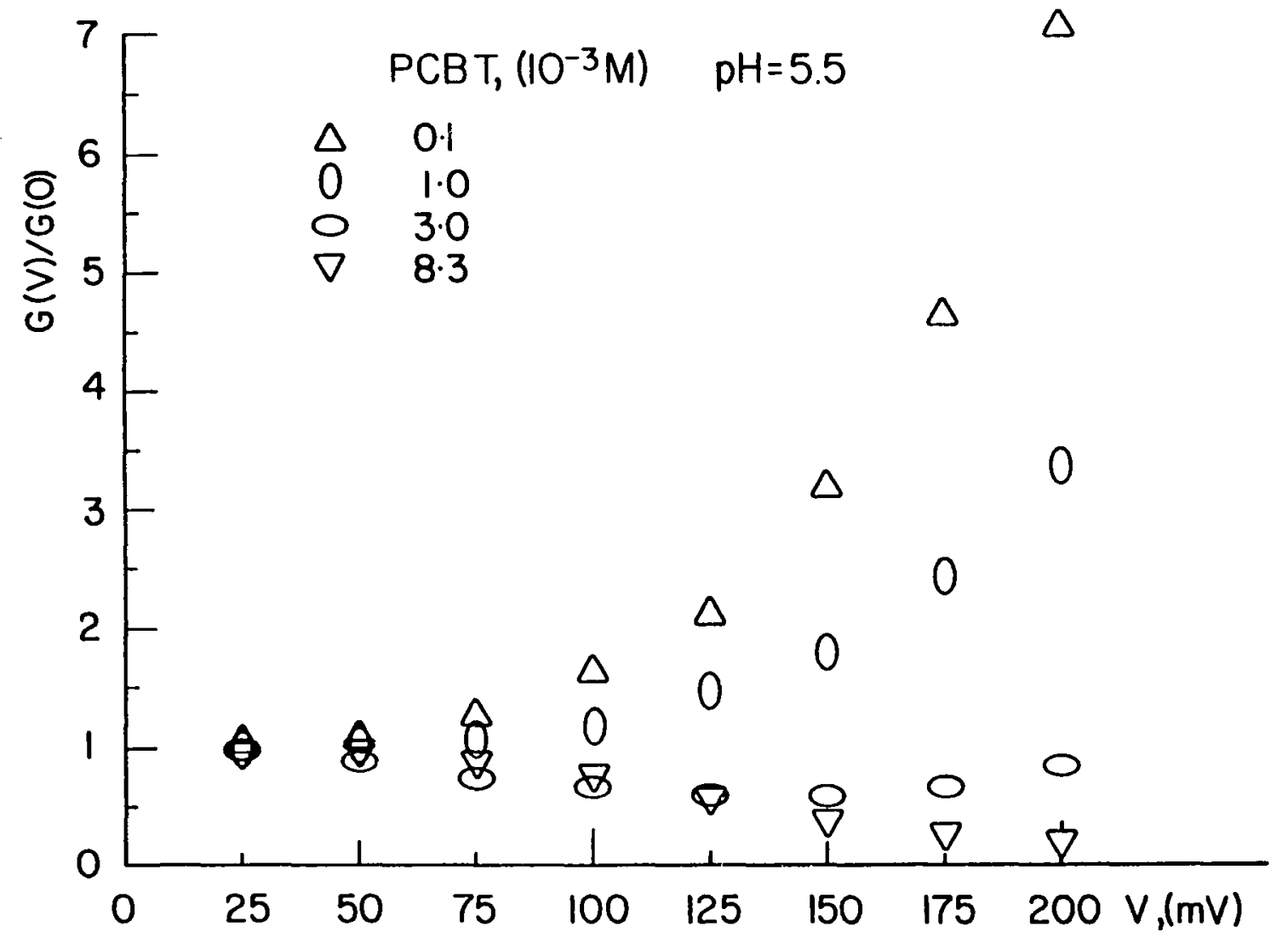

Figure 56. Voltage dependence of the PCBI induced normalized membrane conductance, indicating the development of kinetic limitations at increasing concentration of PCBT. 


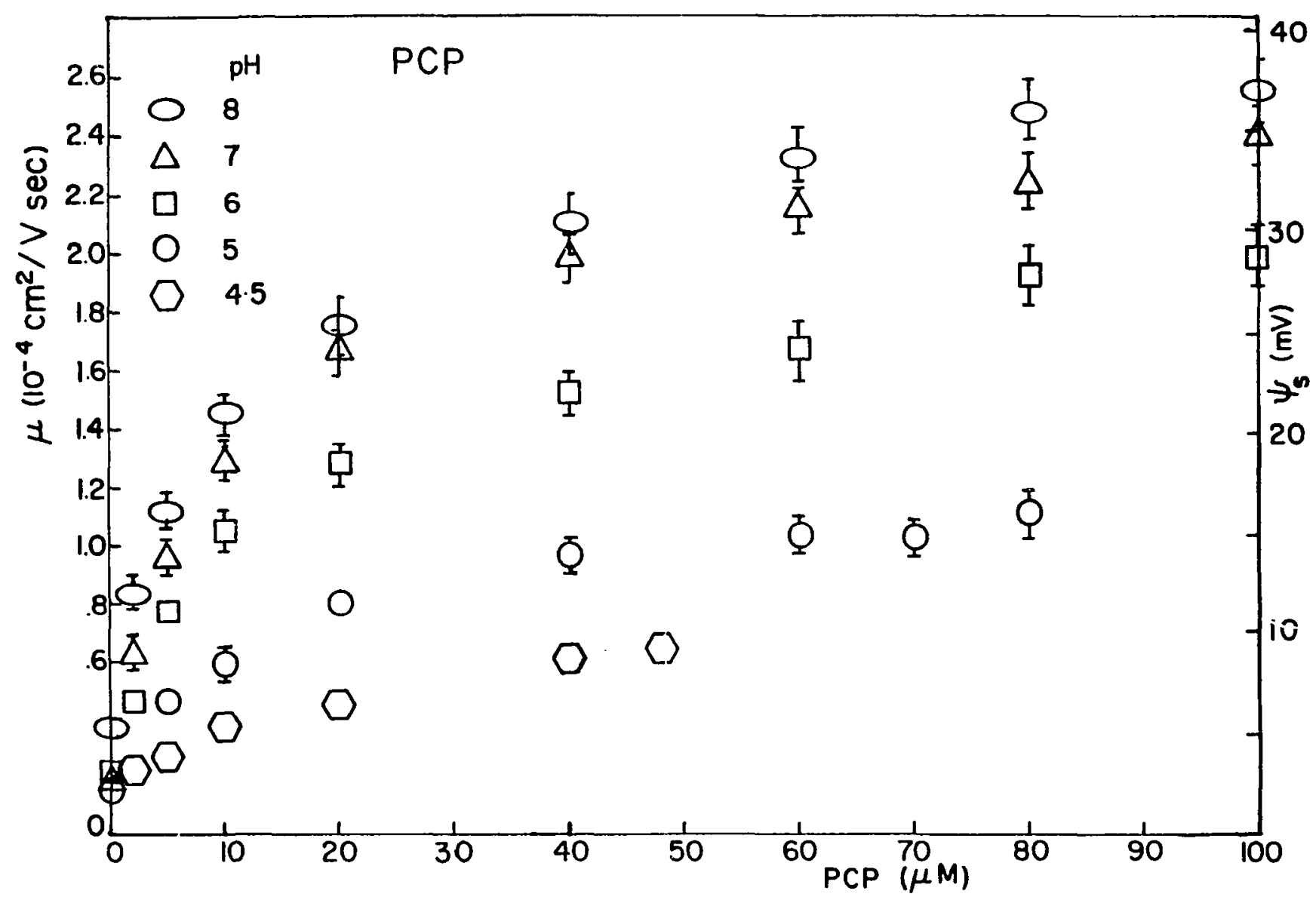

Figure 57. Variation of the electrophoretic mobility and the corresponding electric potential at the membrane surface (zeta potential) of lecithin vesicles, with the aqueous PCP concentration. 


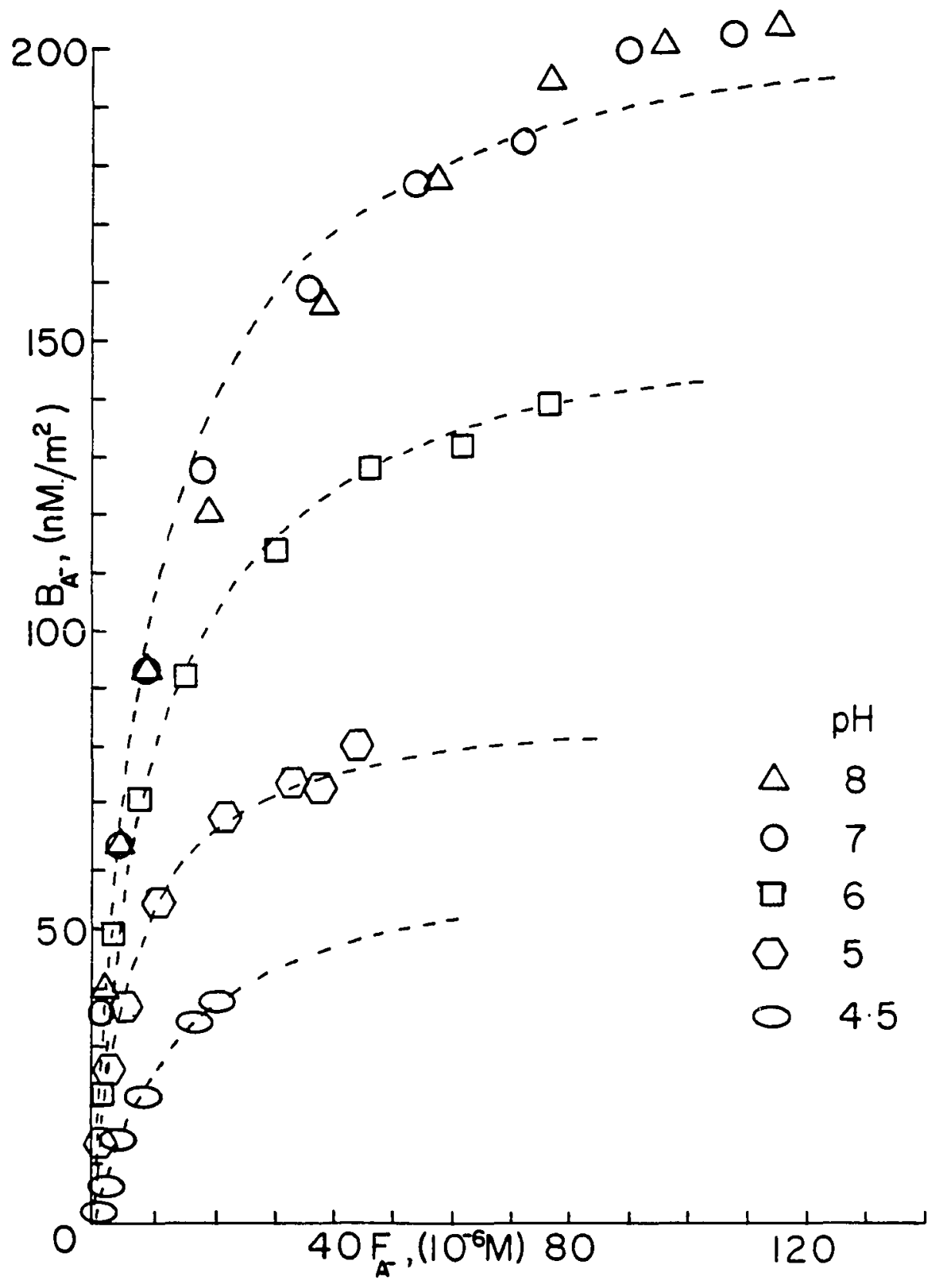

Figure 58. Variation of the density of PCP anions adsorbed to membrane of vesicles $\left(B_{A}\right)$, with the aqueous concentration of $P C P$ anions $\left(F_{-}-\right)$, calculated using $\mathrm{pK}_{a}=4.8$. The dashed Iines represent Langmuir adsorption isotherms. 
vesicles was corrected using the knowledge of the surface area per lecithin molecule in bilayers $(60,61$, and results of experiments of Barbara Sinha done in our lab), and the total amount of lecithin used. However the error due to a similar decrease of aqueous PCP anion concentration due to adsorption of neutral PCP molecules to vesicles was not corrected, because the characteristics of adsorption of neutral PCP molecules to vesicles were not known. The latter correction is expected to be significant only at $\mathrm{pH}$ less than or comparable to the $\mathrm{pK}_{\mathrm{a}}$ of PCP $(=4.8)$, and becomes negligible at high $\mathrm{pH}$ when the majority of PCP molecules are in the anionic form.

According to simple laws of adsorption (Langmuir isotherm) a plot of $B_{A^{-}}-F_{A^{-}}$against $F_{A^{-}}$, where $B_{A}-$ is the adsorbed ion density, and $F_{A}-$ is the free ion density (Scatchard plot) yields a straight line. We found that our experimental results at high $\mathrm{pH}$ obeyed the Langmuir adsorption isotherm only after the aqueous PCP anion concentration adjacent to the membrane has been corrected by the Boltzmann factor, $\exp \left(e \Psi_{s} / k T\right)$ (Eq. 332), where $\Psi_{s}$ is the electric potential at the hydrodynamic plane of shear (62). The anion concentration in the aqueous layer adjacent to the membrane is less than its concentration in the bulk because of electrostatic repulsion between anions adsorbed to the membrane and those in the aqueous layer adjacent to the membrane. Boltzmann factor corrects for the error due to this electrostatic effect. Fig. 59 is a Scatchard plot for the results at pH equal to 7 and 8 , with the free anion density corrected by the Boltzmann factor.

MODIFICATION OF 2,4,5-TRICHLOROPHENOL-INDUCED MEMBRANE CONDUCTIVITY BY TETRAPHENYLARSONIUM AND SALICYLATE IONS

Fig. 60 shows the enhancement and suppression of the 2,4,5-TCP 


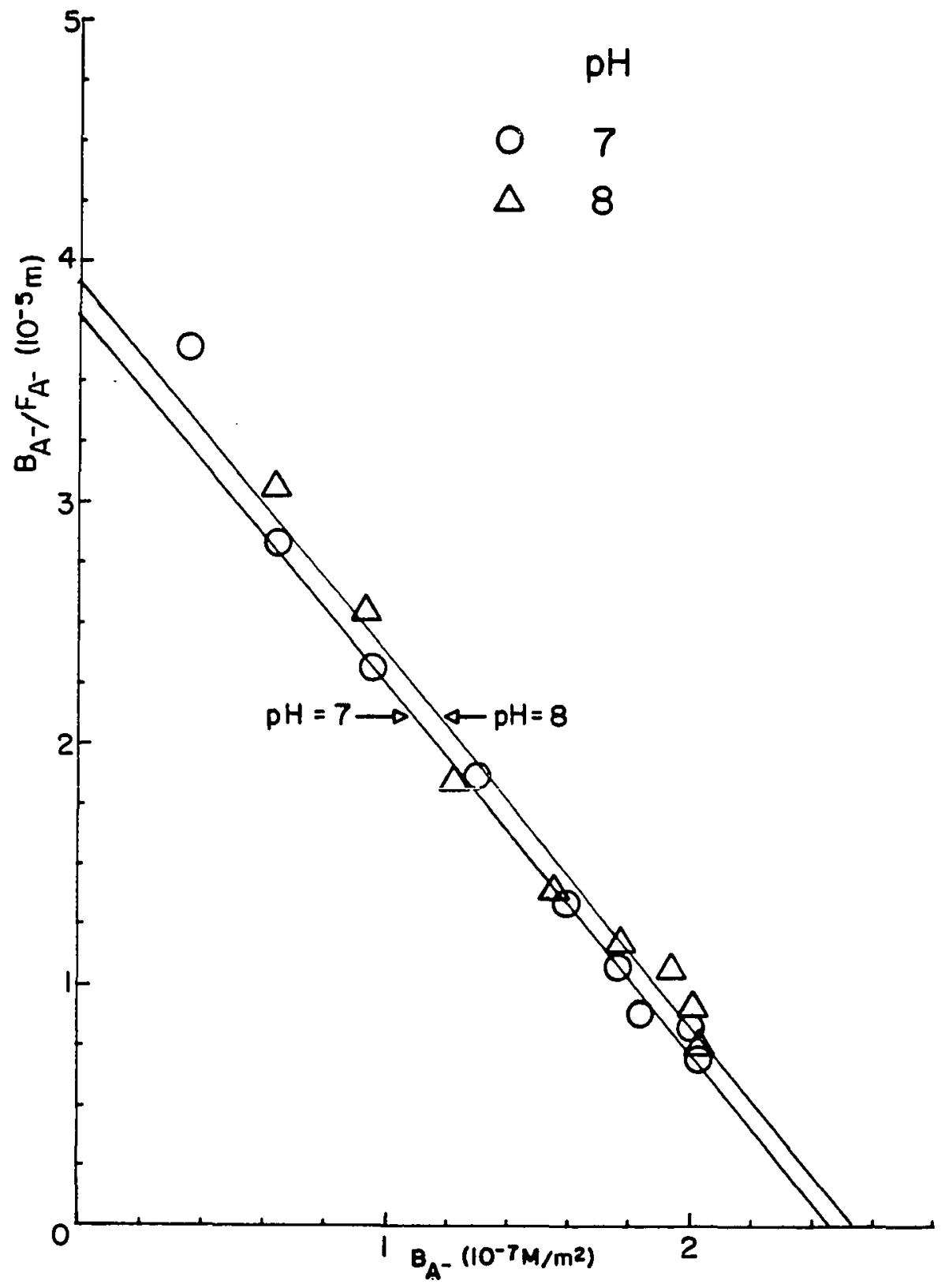

Figure 59. Scatchard plot (see text) for the adsorption of $\overline{P C P}$ anions to membranes. The aqueous PCP anion concentration has been corrected to account for its decrease due to (1) adsorption, and (2) repulsion between adsorbed and free anions. The fit of the data to straight lines indicates that the adsorption of PCP anions to membranes can be well described by Langmuir adsorption isotherm. 


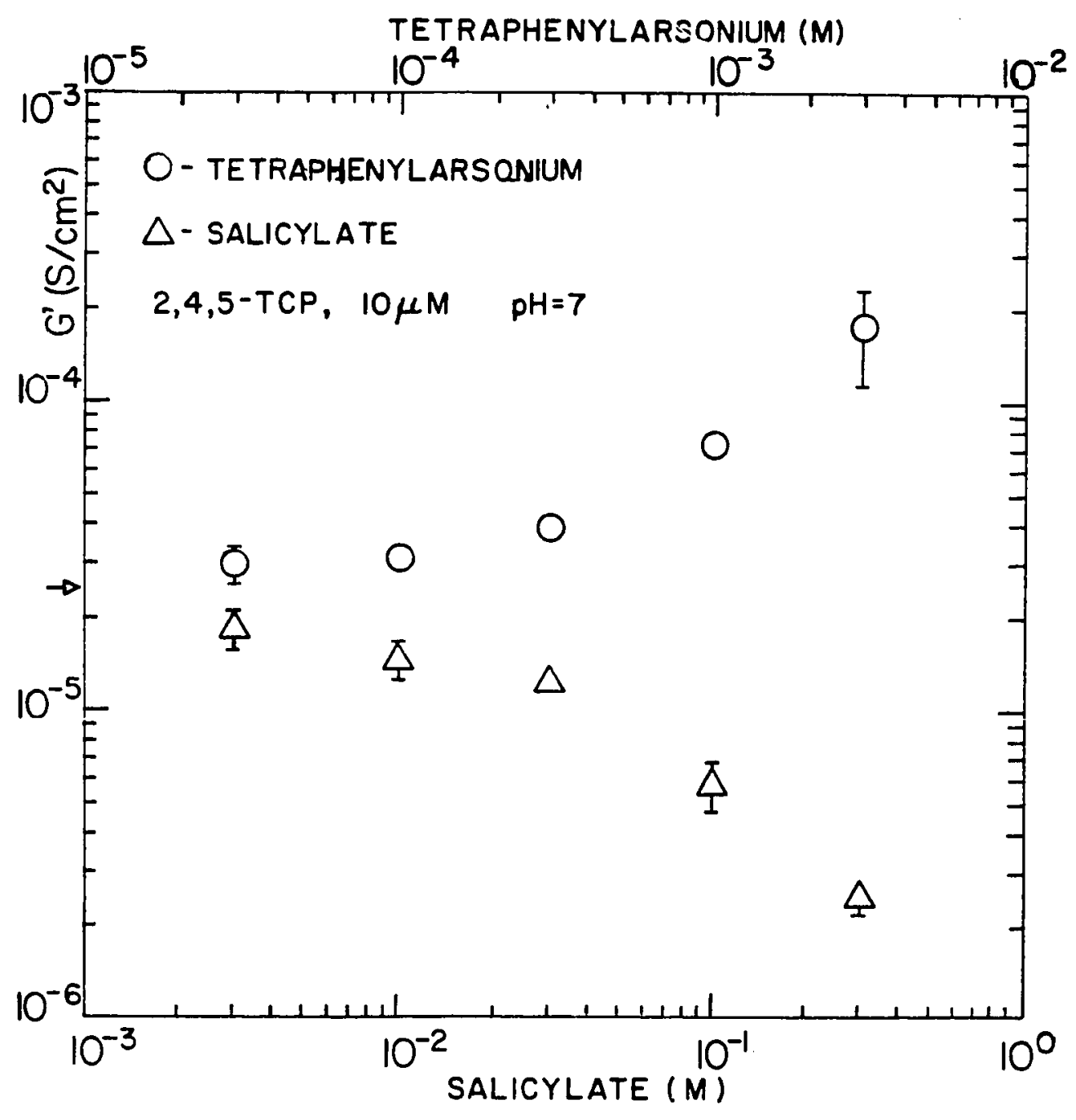

Figure 60. Enhancement due to adsorbed tetraphenylarsonium (circles) and suppression due to adsorbed salicylate (triangles) ions of 2,4,5-TCP induced membrane conductivity. The arrow indicates the conductance level in the absence of any modifier ions. Modifier ions by themselves do not induce any significant increase of the membrane conductivity. 
induced membrane conductivity by adsorbed tetraphenylarsonium and salicylate ions respectively. The arrow indicates the conductivity level in the absence of any modifier ions. We did not observe any significant increase in the steady state conductivity level in the presence of modifier ions alone. Since tetraphenylarsonium and salicylate ions impart a positive and negative potentials to the membrane $(63,64)$, results of Fig. 60 are consistent with the dimer hypothesis $(44,45)$ according to which the membrane permeable ion derived from $2,4,5-\mathrm{TCP}$ is a negatively charged dimer complex $\mathrm{HA}_{2}^{-}$. Since there are no kinetic limitations at this low concentration of $2,4,5-\mathrm{TCP}$, the membrane conductance is limited by diffusion over the membrane potential energy barrier. Under this condition it is possible to determine the electric potential $\Psi_{\mathrm{m}}$ imparted to the membrane by these modifier ions using the equation $G^{\prime}=G \cdot \exp \left(-e \Psi_{m} / \mathrm{kT}\right)(\mathrm{Eq} \cdot 346)(7,28,64)$, where $G^{\prime}$ and $G$ are conductivities in the presence and absence of modifier ions respectively. Fig. 61 is a plot of $\Psi_{\mathrm{m}}$, obtained from the above equation and the results of Fig. 60. At the highest concentration of membrane modifier ions the height of the membrane barrier changed as much as $40 \mathrm{mV}$.

Fig. 62 shows the $\mathrm{pH}$ dependence of $2,4,5-\mathrm{TCP}$ induced conductance, (a) in the presence of $3 \mathrm{mM}$ tetraphenylarsonium ions, (b) in the presence of $0.3 \mathrm{M}$ salicylate ions, and (c) in the absence of any modifier ions. Recently Stark (65) has suggested that hydrophobic anions like tetraphenylborate $\mathrm{T}^{-}$act as carriers for the tetraphenylarsonium $\mathrm{B}^{+}$cations by the formation of neutral ion pairs $\mathrm{T}^{-} \mathrm{B}^{+}$. Since in Fig. 62 the shapes of the conductivity curves are not changed by the presence of modifier ions, we conclude that the processes similar to those reported by stark (65) do not alter the mechanism of 2,4,5-TCP 


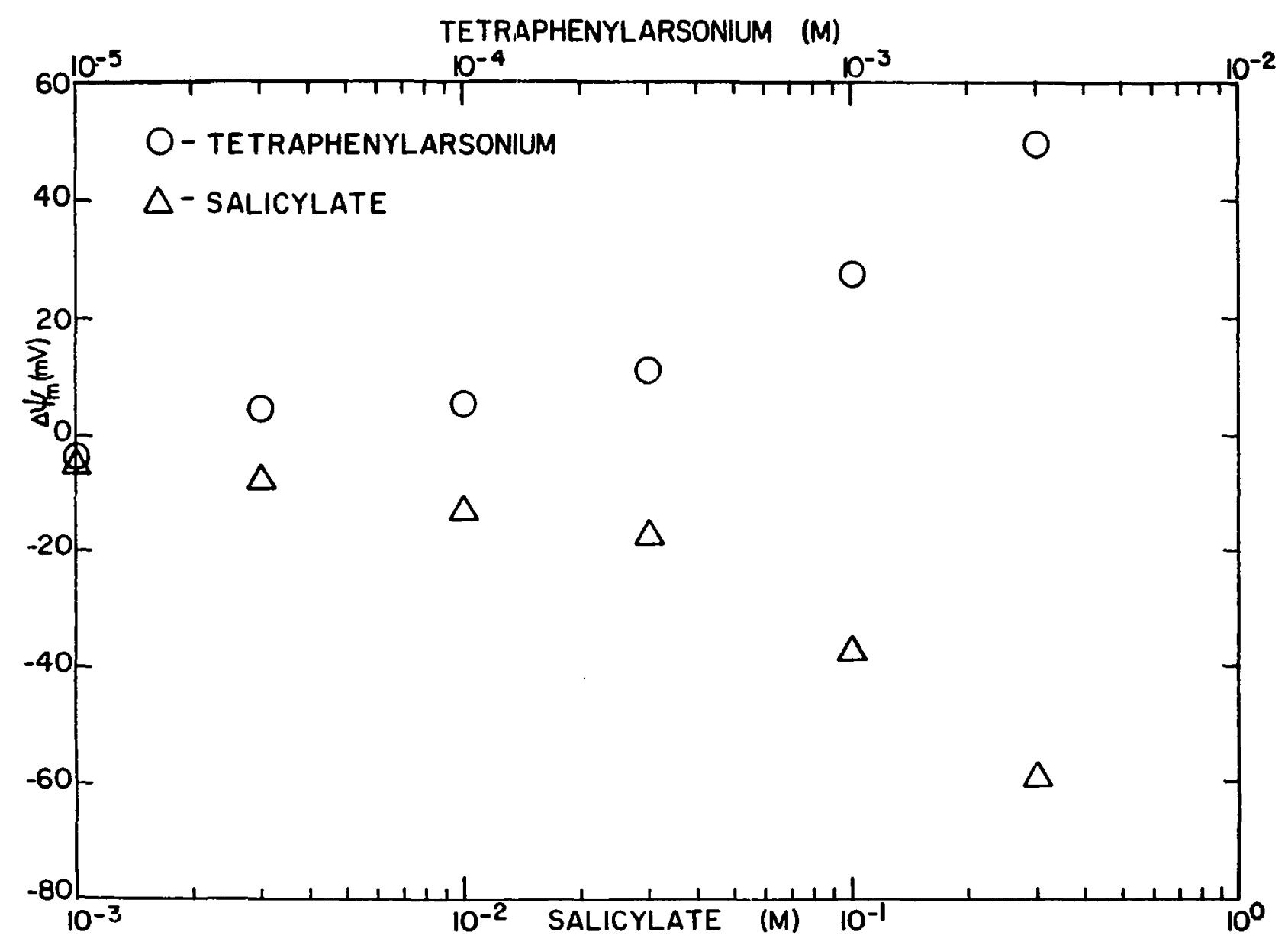

Figure 61. Variation of the membrane potential induced by adsorbed tetraphenylarsonium (circles), and salicylate (triangles) ions, calculated using Eq. 346 and results of Fig. 60 , with increasing concentration of modifier ions. 


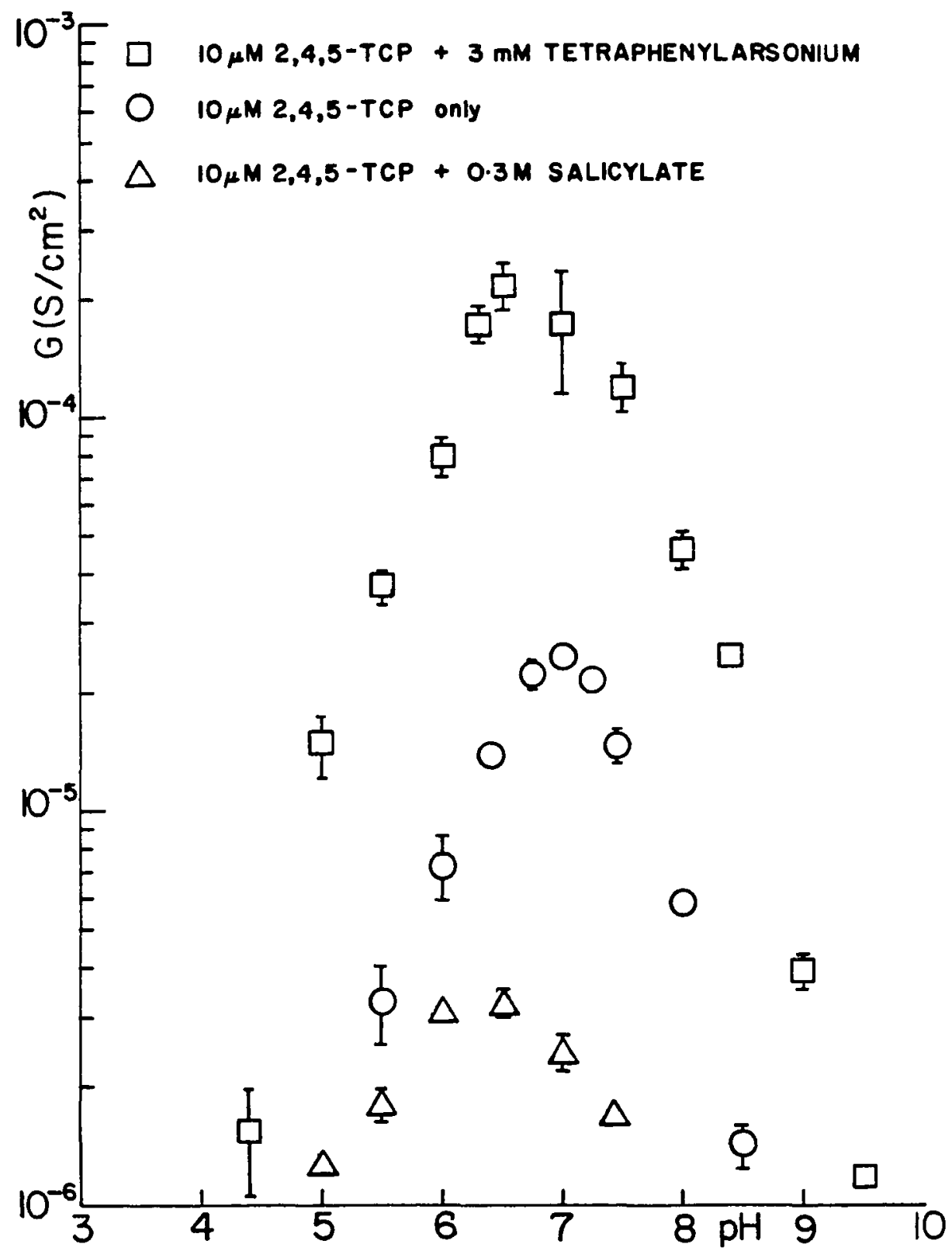

Figure 62. $\mathrm{pH}$ dependence of 2,4,5-TCP induced membrane conductance in the presence of membrane modifier ions; tetraphenylarsonium-squares, salicylate-triangles, and in the absence of modifier ions-circles. Although the $\mathrm{pH}$ of the maximum conductivity shifts to lower $\mathrm{pH}$ in the presence of modifier ions the shape of the curve remains unchanged. 
induced membrane conductivity. The shift of the conductivity maximum to less than the $\mathrm{pK}_{\mathrm{a}}$ of $2,4,5-\mathrm{TCP}$ (which is 7.0 ) could be explained for the case of tetraphenylarsonium by electrostatic arguments (see Chapter V), since tetraphenylarsonium ions impart a positive electric potential to the membrane. However, the shift of the conductivity maximum to $\mathrm{pH}$ below the $\mathrm{pK}_{\mathrm{a}}$ of $2,4,5-\mathrm{TCP}$ due to salicylate ions is not in agreement with the same electrostatic argument. The reason for the discrepancy is not known, but some hypotheses pertinent to this topic are proposed in Chapter V.

STUDIES OF MEMBRANE CURRENT RELAXATION

Fig. 63 shows the variation of the time constant and the relaxation amplitude with voltage for $\mathrm{PCBT}$ induced current relaxations under a voltace step. These variations agree with those predicted by dimer models (Fig. 34). We observed that the relaxation amplitude and the time constant are strongly voltage dependent for the case of PCBT but not so for the cases of $\mathrm{PCP}$ and 2,4,5-TCP. From the relation between the relaxation amplitude $\alpha$ and the kinetic limitation parameter E, $\alpha=E \cdot \cosh (e V / 2 \mathrm{kT})$, derived in Chapter III (Eqs. 244,323) for the dimer models, a strong dependence of $\alpha$ on voltage $V$ indicates that the kinetic limitations are more pronounced (i.e. large E) for PCBT-induced conductivity, compared to that induced by PCP or 2,4,5-TCP. This is in agreement with the same conclusion drawn before from the voltage dependence of steady state normalized membrane conductivities, and is also consistent with the observation of higher conductivity for PCBT.

Fig. 64 shows the increase of the time constant with pH for PCP induced current relaxations, and this variation is in agreement with 


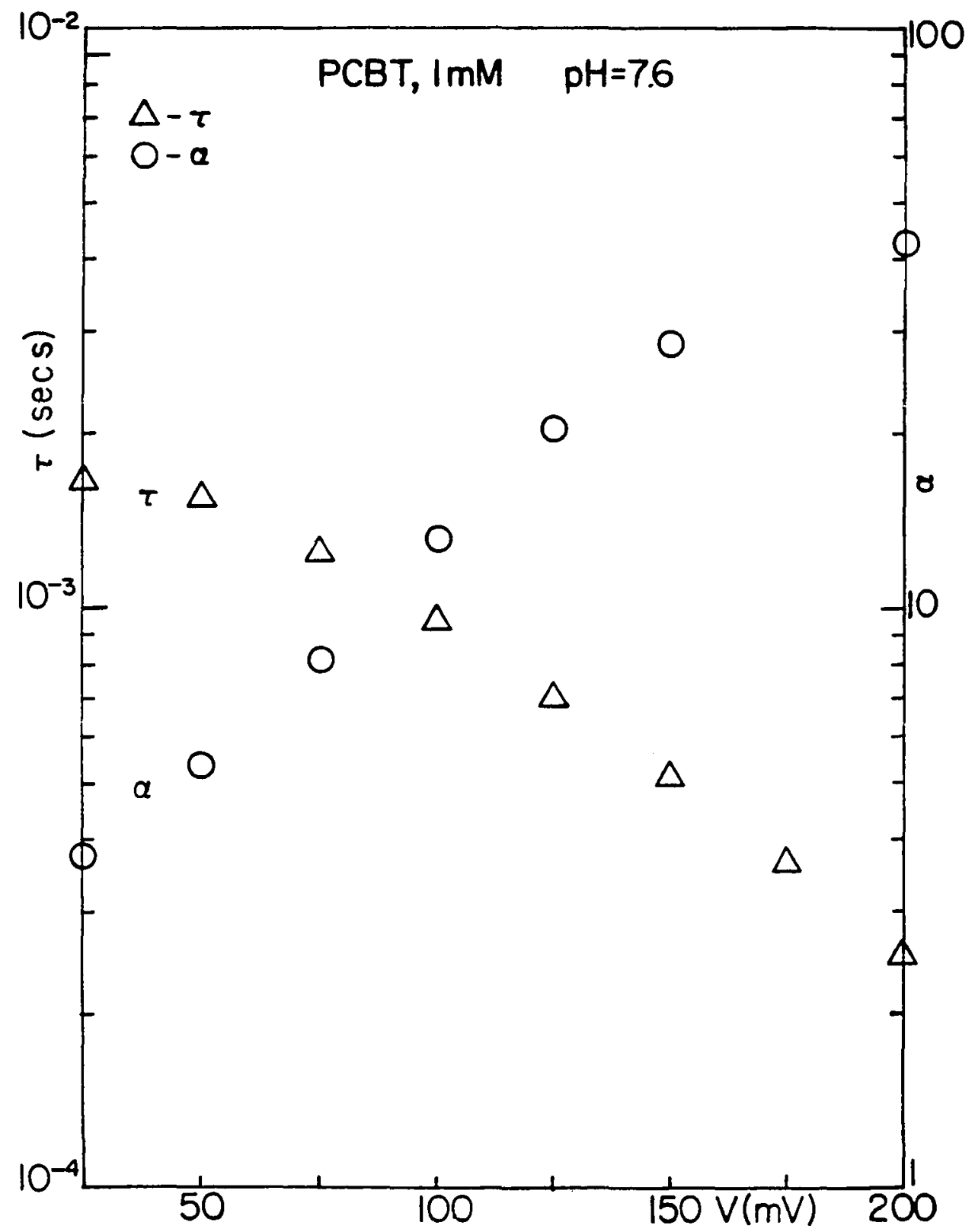

Figure 63. Variation of the relaxation time constant $T$ and the relaxation amplitude $\alpha$ with the voltage for PCBT induced current relaxations undor a voltage step. The strong voltage dependence of $\alpha$ indicates the existence of strong kinetic Iimitations for PCBT induced membrane conductance. The curves are similar to those predicted by theory (Figure 34 ). 


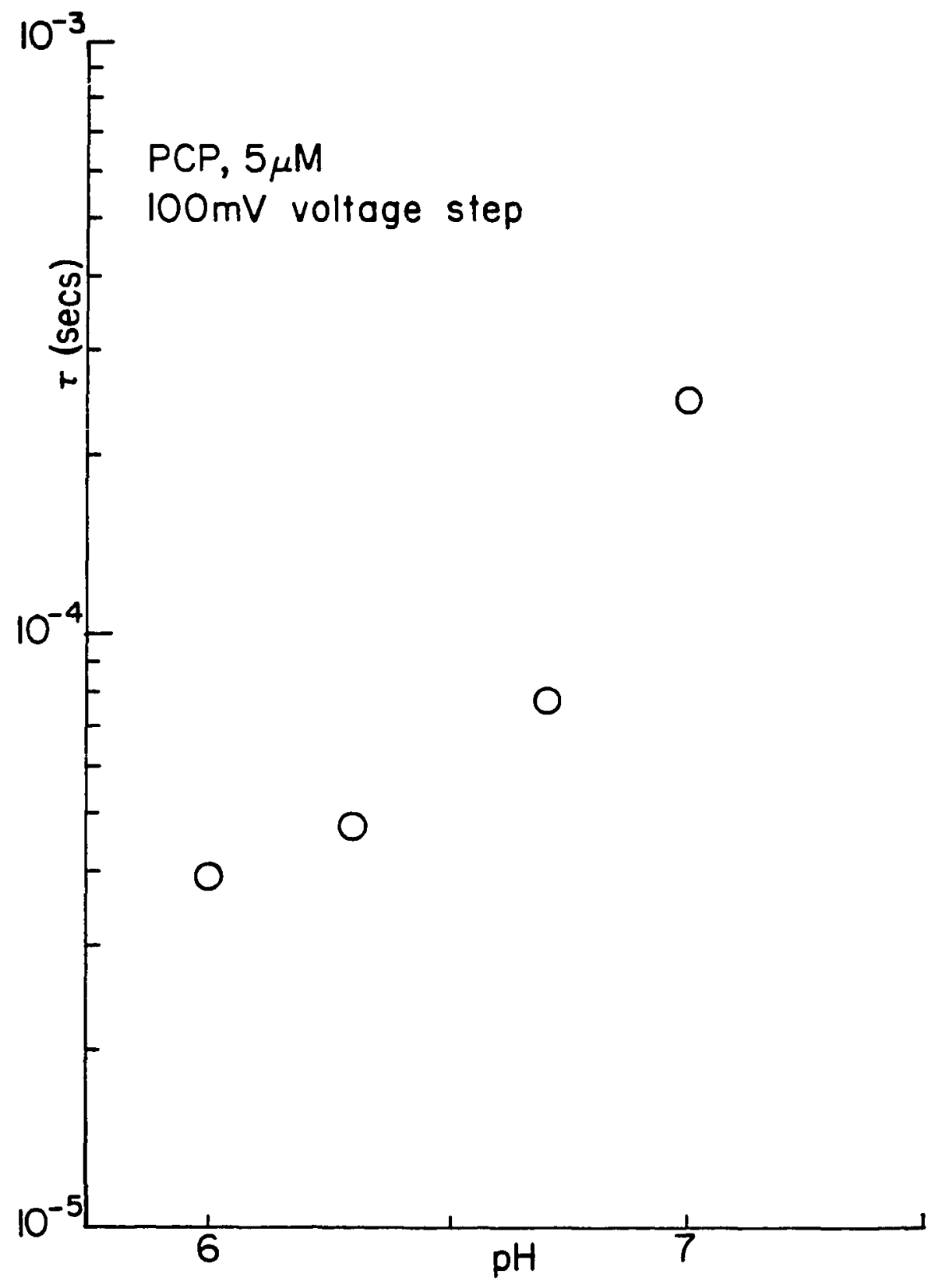

Figure 64. Increase of the time constant with the $\mathrm{pH}$ for PCP induced current relaxations for a $100 \mathrm{mV}$ voltage step. The variation is similar to that predicted by theory (Fig. 35). The observation that the relaxations are $\mathrm{pH}$ dependent indicates that the formation and dissociation of the membrane permeable ion does not limit the transmembrane flux. 
the predictions of dimer models (Fig. 35). All three compounds PCP, $\mathrm{PCBT}$, and, 2,4,5-TCP exhibited $\mathrm{pH}$ dependent current relaxations at $\mathrm{pH}$ greater than the $\mathrm{pk}_{\mathrm{a}}$ of the uncoupler. The fact that the relaxations are $\mathrm{pH}$ dependent indicates that the observed relaxations are not due to the slow dimerization process, because the kinetic limitation due to slow dimerization is independent of the pH. According to dimer models A and $B$ (Chapter III) the relaxations observed at high $\mathrm{pH}$ could arise due to either (a) slow backflow of HA molecules, (b) slow proton association/dissociation reaction $\mathrm{HA}=\mathrm{H}^{+}+\mathrm{A}^{-}$, or (for dimer model $\mathrm{B}$ only) (c) due to slow interfacial transfer of HA molecules. Since the variations of the relaxation characteristics with the pH are similar for the above mentioned kinetic limitations (Fig. 35), it is not possible to distinguish, based on the limited steady state and relaxation data, which type of kinetic limitation is responsible for the relaxations observed at high $\mathrm{pH}$.

Fig. 65 shows the decrease of the time constant with uncoupler concentration for $2,4,5-\mathrm{TCP}$ induced current relaxations, and this variation is in agreement with the predictions of dimer models (Fig. 36 ). Experimental results presented in Fig. 65 do not make it possible to differentiate between the types of kinetic limitations because the variation of the relaxation characteristics with concentration of the uncoupler is similar for several types of kinetic limitations under consideration (see Fig. 36). However, the fact that the relaxation time constant is dependent on concentration of the uncoupler indicates that the observed relaxations are not due to a slow dimerization process, because this kinetic limitation is independent of uncoupler concentration. 


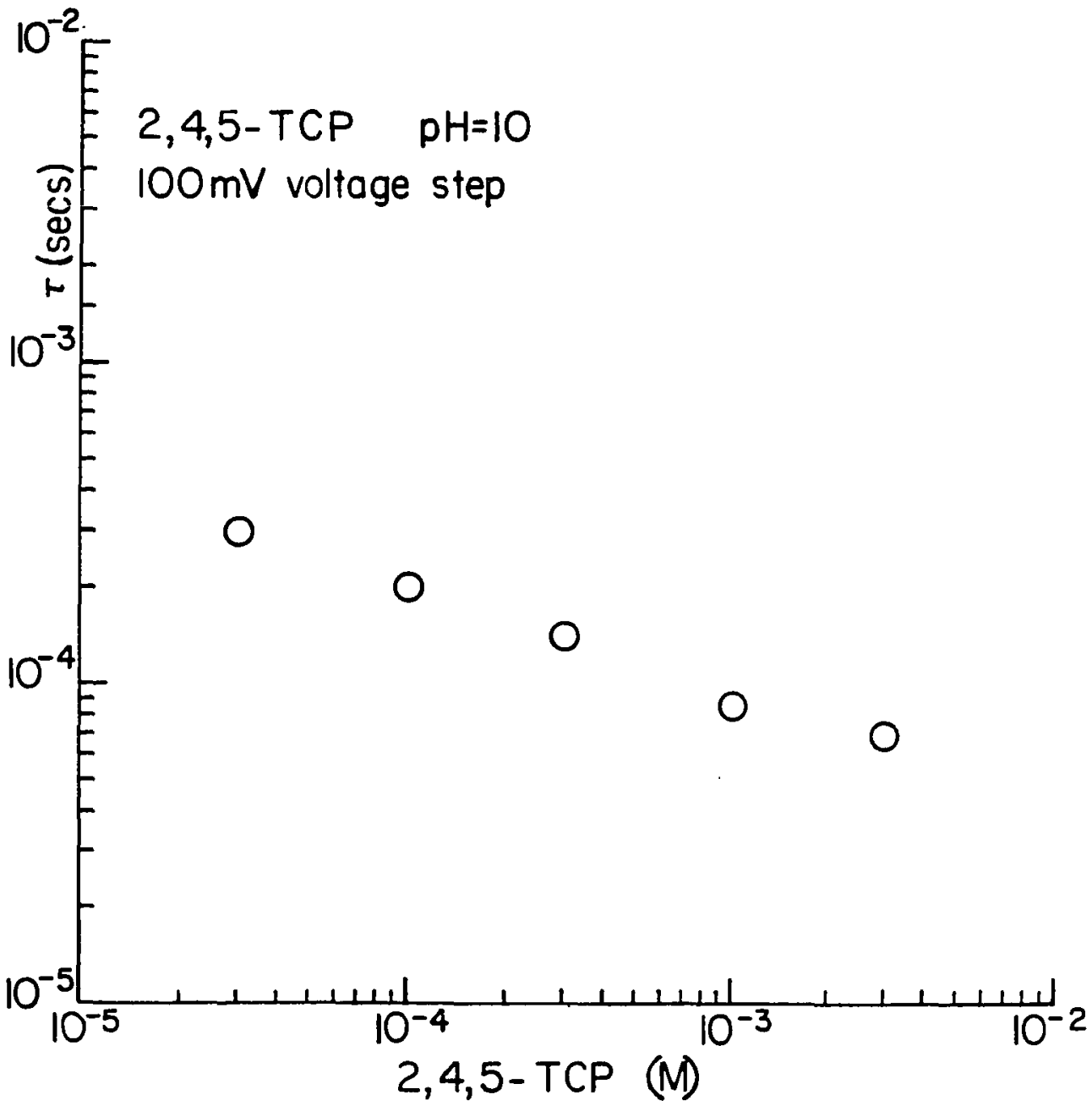

Figure 65. Decrease of the time constant with the uncoupler concentration for $2,4,5-\mathrm{TCP}$ induced current relaxations. The variation is similar to that predicted by theory (Fig. 36). The observation that current relaxations are concentration dependent indicates that the slowest process in the membrane transport kinetic scheme is not the dimer formation/dissociation step. 
TOXICITY OF PENTACHLOROPHENOL TO ALGA

The toxicity of PCP to the alga Selenastrum capricornutum was measured by the decrease of $\mathrm{C}^{14}$ fixation by these organisms in the presence of PCP. Fig. 66 shows the decrease of the rate of $\mathrm{C}^{14}$ uptake by the alga with increasing concentration of PCP. The arrow corresponds to the rate of carbon uptake in the absence of PCP.

The alga was exposed to PCP for about two hours. The occurrence of toxic effects on such a small time scale indicates that the effect is not related to changes of algal metabolism which occur on a time scale of 10 hours. In addition, the toxic effect occurred only in the presence of light, which indicates that PCP affects the light driven reactions of the photosynthetic process. It has been suggested for other uncouplers (66-69), that the reduction of carbon uptake is the result of depletion of the energy source, ATP, required for driving the carbon uptake. It is expected that PCP has the same effect. Depletion of ATP pool occurs as a result of uncoupling the photosynthetic phosphorylation process by PCP.

Fig. 66 shows that the toxic effect begins to appear at a PCP concentration of about $1 \mu \mathrm{M}$ and is fully developed at $10 \mu \mathrm{M}$ PCP. The PCP induced lipid bilayer membrane conductance begins to increase above the background at about $1 \mu \mathrm{M}$ PCP and the conductance is almost saturated at about $10 \mu \mathrm{M}$ PCP (ref. Fig. 2 of ref. 28). The correlation that we observed between the concentration of PCP at which the toxicity occurredin the alga and the conductance was induced in lipid bilayer membranes, and the rapid response of the alga to PCP, are consistent with the hypothesis that the mechanism of uncoupling is identical to 


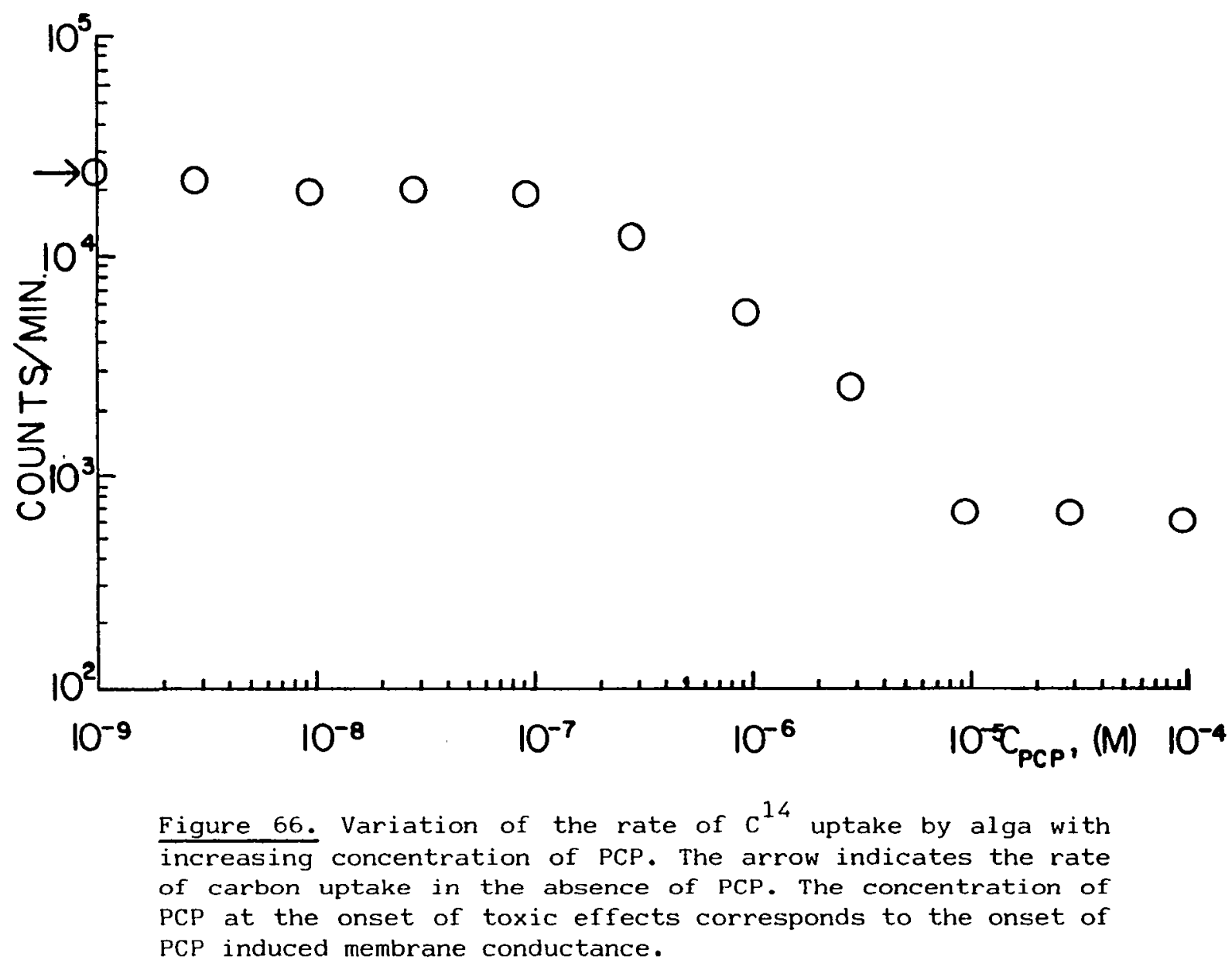


the mechanism of charge transport in lipid bilayer membranes induced by weak acids.

We found that the alga by itself, in the absence of uncoupler has a characteristic pH dependence of carbon uptake (Fig. 67). This finding complicates any pH-related studies of the effect of uncouplers, analogous to those done with bilayers. Results similar to ours were observed by Werdan et. al. (66) for spinach chloroplasts when the rate of carbon fixation was plotted as a function of the $\mathrm{pH}$ of the stroma (Fig. 5 of ref. 66). In their report the maximum rate of carbon uptake occurred at stroma $\mathrm{pH}$ of about 8.0 .

If the bell shaped curve obtained for the $\mathrm{pH}$ dependence of carbon uptake is due to limitation of carbon availability then these observations are consistent with the hypothesis that $\mathrm{HCO}_{3}^{-}$is the source of carbon required by the alga. We could reproduce the $\mathrm{pH}$ dependence of carbon fixation rate with the assumption that the rate is proportional to $\mathrm{HCO}_{3}^{-}$concentration. The maximum rate then corresponds to the proton concentration equal to $\sqrt{\mathrm{K}_{1} \cdot \mathrm{K}_{2}}$, where $\mathrm{K}_{1}$ and $\mathrm{K}_{2}$ are the first and second dissociation constants of $\mathrm{H}_{2} \mathrm{CO}_{3}$. The predicted maximum corresponds to a $\mathrm{pH}$ of 8.3 and agrees with the value of about 8 observed by Werdan et. al. (66) for spinach chloroplasts. The occurrence of maximum carbon uptake for alga Selenastrum capricornutum at a pH of about 6 (Fig. 67) rather than 8 could be due to the existence of $\mathrm{pH}$ gradients across the outer membranes of the alga. Such pH gradients corresponding to higher $\mathrm{pH}$ of the stroma with respect to the outer aqueous phase have been reported in the literature (66). 


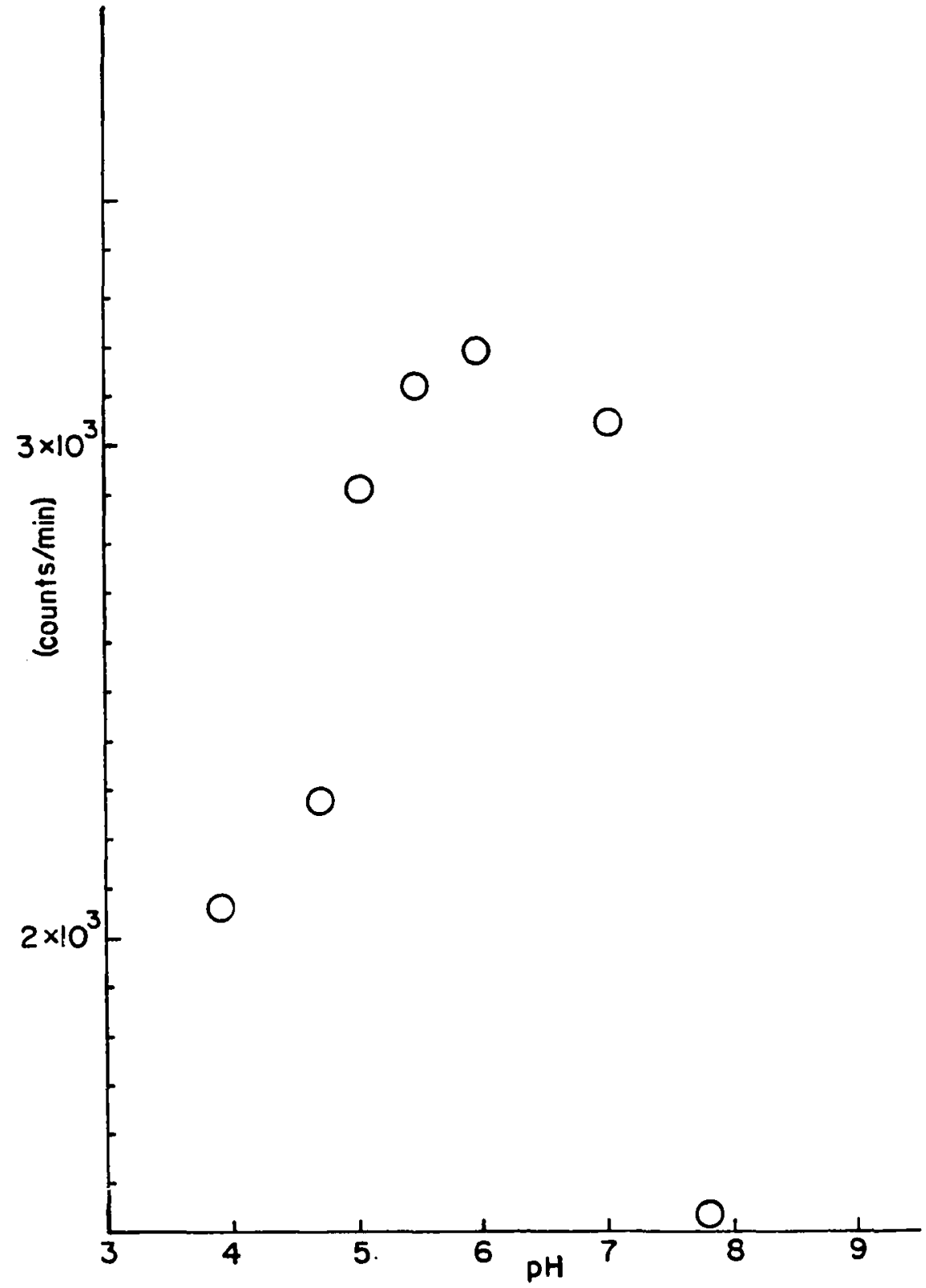

Figure 67. Characteristic pH dependence of the rate of carbon uptake by alga in the absence of PCP. If this variation is due to limitation of carbon availability, then these observations are consistent with the hypothesis that $\mathrm{HCO}_{3}^{-}$is the source of carbon required by the chloroplasts. 
CHAPTER V

DISCUSSION

\begin{abstract}
In addition to the discussion of main experimental and theoretical results, we will attempt to develop a comprehensive overview of the mechanism of induced hydrogen ion transfer in lipid membranes in the presence of chlorinated phenols. We present first the mechanistic picture of the transport processes involved and then arguments in support of the applicability of selected kinetic schemes, and in conclusion we point out the biological significance of the results obtained.
\end{abstract}

MAIN FEATURES OF THE MECHANISM OF MEMBRANE CONDUCTION INDUCED BY $2,4,5-T R I C H L O R O P H E N O L$, PENTACHLOROPHENOL, AND PENTACHLOROBENZENETHIOL

\begin{abstract}
One of our observations was that an electrical potential difference developed in treated membranes in the presence of hydrogen ion concentration gradients. The potential distribution was such that the potential of the low $\mathrm{pH}$ side was negative with respect to the high $\mathrm{pH}$ side. The magnitude of the potential difference was approximately $50 \mathrm{mV}$ per decade of hydrogen ion activity gradient. In membrane biology it has been often incorrectly implied that the polarity of membrane potential difference indicates the polarity of membrane permeable ions. Superficially, our data would suggest that the above mentioned compounds also induce membrane permeability of $\mathrm{H}^{+}$or $\mathrm{OH}^{-}$ions. Induced permeability of hydrogen ions was often invoked or implied in earlier
\end{abstract}


literature on biological action of uncouplers of oxidative phosphorylation $(70,71)$. The above mentioned chlorinated phenols belong to this category $(32,72-78)$. Indeed, from the condition of thermodynamic equilibrium for hydrogen ions it follows that $v^{\prime \prime}-v^{\prime}=(k T / e) \ln \left(a_{H}^{\prime} / a_{H}^{\prime \prime}\right)$, where $a_{H}$ is the activity of hydrogen ions in the aqueous phases surrounding the membrane. Thus, for $a_{H}^{\prime}>a_{H}^{\prime \prime} v^{\prime}-v^{\prime \prime}<0$, in agreement with our experimental results (Fig. 48). But it would be incorrect to conclude that hydrogen ions alone permeate across lipid bilayers.

It was pointed out recently (30) that if chemical reactions, such as association-dissociation processes involving hydrogen ions, are linked to the process of charge transfer across the membrane, the membrane potential data are not useful for the purpose of discrimination among different kinetic models. For the same reason our experimental membrane potential results are compatible with the predictions of models in which both the anion $\mathrm{A}^{-}$, produced by the dissociation of the chlorinated phenol molecule $H A$, and $H A$ itself are assumed to be membrane permeable. Thus, the candidates for electric current carriers in the membrane are hydrogen and hydroxide ions, and both the neutral and ionized chlorinated phenol molecules. It follows from thermodynamic arguments (79), that the probability of permeation of hydrogen and hydroxide ions without assistance across nonpolar membrane core is very small, so that the phenol molecules must be directly or indirectly involved in the transmembrane charge transfer.

It should be realized that the experimental membrane conductance data shown in Figs. $42-47$ actually reflect both the conductance of the membrane and of the adjoining electrolyte layers. One of the main tasks in elucidating the membrane conduction mechanism is to 
determine the identity of membrane permeable ions and to find out the location of their formation. In our case it is desirable to establish first whether the membrane permeable ions have their origin in the aqueous medium surrounding the membrane or in the membrane itself. Some insight into this problem was gained from the analysis of the steady state conductance results. If the membrane permeable ions were formed in the bulk aqueous solution, then the maximum flux the membrane/electrolyte system can support would be determined by the maximum diffusion flux in the aqueous medium. Under most experimental conditions used the Debye Huckel length (59) of the electrolyte/membrane interface is on the order of $10 \mathrm{~nm}$ or less whereas the thickness of unstirred layers is $.05-.6 \mathrm{~mm}(80,59)$. The maximum possible conductance of membrane/ electrolyte system can be estimated from the maximum ion flux across two unstirred layers. Thus, if the membrane permeable ions were formed in the aqueous medium, then the steady state conductance limit, obtained from the condition of flux continuity across the membrane and the unstirred layers, is equal to

$$
G_{1 i m}=e^{2} D_{\omega} n / 2 \delta k T
$$

where $D_{\omega}$ is the diffusion coefficient of membrane permeable ion in water, $n$ the volume density of membrane permeable ions in the aqueous medium, and $\delta$ the thickness of the unstirred layer. For $D_{\omega}=7 \times 10^{-6}$ $\mathrm{cm}^{2} / \mathrm{s}$, a value typical for the size of phenol molecules, $\delta=0.2 \mathrm{~mm}$ (5) and $\mathrm{n}$ corresponding to $1 \times 10^{-4} \mathrm{M}$ (an upper limit on aqueous concentration), the estimated maximum conductance is $7 \times 10^{-5}$ siemens $/ \mathrm{cm}^{2}$. The experimental value of the conductance was found to be 
greater by a factor of four to seven. Moreover, the value of the experimental conductance was rather insensitive to the rate of stirring, which controls the thickness $\delta$, and steady state conductance was attained in less than one second. Both effects are not compatible with the idea of conduction limited by the diffusion of membrane permeable ions, derived from phenols, toward and away from the membrane. The above mentioned facts strongly suggest that membrane permeable ions do not originate in the aqueous medium, but in or at the membrane. Another observation consistent with the latter was that addition of phenols to the membrane forming solution resulted in immediate attainment of stable conductance, whereas incorporation from the aqueous medium containing chlorinated phenol resulted in a gradual development of conductance with time.

All three investigated compounds exhibited similar dependence of conductance on hydrogen ion activity (Figs. 42-44). Also there were ranges of experimental conditions in which the conductance increases approximately as a second power of the concentration of the phenol. These two features are in support of applicability of the dimer hypothesis of membrane transport $(44,45)$ according to which the negatively charged dimer complex, a product of recombination of one neutral and one ionized molecule of the chlorinated phenol, is the membrane permeable ion responsible for the conduction effect. According to this hypothesis the conductance corresponding to equilibrium situation is $(44)$

$$
G \propto \frac{a_{H}}{\left(k_{a}+a_{H}\right)^{2}} N_{0}^{2},
$$


where $K_{a}$, $a_{H}$ and $N_{0}$ are dissociation constant, hydrogen ion activity and concentration of membrane modifier in membrane, respectively. The first factor qualitatively reproduces the $\mathrm{pH}$ dependence, the second factor the concentration dependence of our experimental results under certain conditions. More detailed discussion of the conduction mechanism will be presented later in this section.

It was concluded that the membrane permeable ions are not formed in the aqueous medium, but in the membrane. In order to complete the conceptual development of the model of the conduction mechanism it is necessary to elucidate the charge transport in the aqueous phase surrounding the membrane. For that purpose we used the membranes treated with PCBT, because these were highly conductive, and it was found that if the aqueous medium contained low concentration of buffer, i.e. when the hydrogen ion storage and release system had a small capacity, the steady state conductance was significantly reduced (Fig. 49). In addition, under those conditions we observed a slow transient current after the application of the potential difference across the membrane. This slow transient process can be understood. It was shown by Neumcke (47) that if the transmembrane current is limited by diffusion in aqueous layers, then the current in the system is

$$
I(t)=z F c_{i}(D / \pi t)^{\frac{1}{2}} \tanh (z e V / 2 k T)
$$

Our experimental results on the time evolution of the current at low buffer capacity agree with the above predicted time dependence (Fig. 50). The above mentioned observations indicate that the conduction current in the aqueous layers adjacent to the membrane is carried by hydrogen and buffer ions. 


\section{VALIDITY OF SMALL DIMER DENSITY APPROXIMATION}

It was shown that membrane conductance induced by chlorinated phenols is compatible with the concept of dimers $\mathrm{HA}_{2}^{-}$as membrane permeable ions. Applicability of the dimer hypothesis rested on an observed proportionality between the membrane conductance and the function $h /(1+h)^{2}$, where $h$ is the reduced hydrogen ion concentration. The dimer hypothesis was supported by the fact that complexes containing $\mathrm{HA}_{2}^{-}$as counterions have been actually isolated (46).

In the theoretical treatment of dimer kinetic schemes we obtained manageable analytical solutions for transmembrane charge flux (Eqs. 223, 292) under the assumption of low density of dimers as membrane permeable ions. This assumption was experimentally tested using the following line of reasoning. If $\mathrm{HA}_{2}^{-}$dimers were the dominant charged species at membrane surfaces, then the surface charge density determined from electrophoretic mobility of lipid vesicles, would be also proportional to dimer density, i.e. to the function $h /(1+h)^{2}$. Instead, we found that the mobility of vesicles is porportional to $(1+h)^{-1}$, indicating that the membrane surface charge is proportional to $\mathrm{A}^{-}$ions adsorbed at the membrane rather than to $\mathrm{HA}_{2}^{-}$. Therefore the density of dimers $\mathrm{HA}_{2}^{-}$at the membrane surface have to be smaller than that of $\mathrm{A}^{-}$; an experimental result in support of the applicability of small dimer density approximation.

\section{BINDING OF PENTACHLOROPHENOL IONS TO PHOSPHATIDYLCHOLINE BILAYERS}

We have shown that it is appropriate to describe the adsorption of pentachlorophenol ions at bilayer membrane surfaces by the Langmuirtype adsorption model, if one takes into account the repulsive electro- 
static interaction between the adsorbed and free pentachlorophenol ions. Our electrophoretic mobility data indicated that such correction may be necessary, since already at moderate concentrations of PCP ( 30 $\mu \mathrm{M}$.$) , the magnitude of electric potential at the shear plane, located$ several angstroms in front of the membrane surface, becomes comparable to $\mathrm{kT} / \mathrm{e}$. The agreement between the experimental values of vesicle mobilities and aqueous PCP anion concentration became consistent with the Langmuir adsorption model only after the introduction of Boltzmann factor $\exp \left(-z e \Psi_{s} / k T\right)$, where $\Psi_{s}$ is the electric potential at the shear plane. The Boltzmann factor takes into account the distribution of PCP anions between the aqueous part of the membrane/water interface and the bulk aqueous phase. The density of PCP anions on the membrane surface $B_{A}$ is then given by

$$
B_{A}=K^{A}\left(B_{T}-B_{A}\right) F_{A} \exp \left(-e \Psi_{s} / k T\right)
$$

which represents the Langmuir adsorption isotherm with the electrostatic correction (23). $F_{A}$ represents trie free anion concentration and $B_{T}$ the total number of binding sites per unit area on the membrane. $K^{A}$ is the binding constant.

From the fit of the above equation to the experimental results, we have obtained the partition coefficient of PCP anion into lecithin bilayers, $\mathrm{K}^{\mathrm{A}} \cdot \mathrm{B}_{\mathrm{T}}=3.8 \times 10^{-5} \mathrm{~m}$, which is about the same $\left(4 \times 10^{-5} \mathrm{~m}\right)$ found for another potent uncoupler of oxidative phosphorylation, TTFB (9). ${ }_{\mathrm{T}}$, the density of $\mathrm{PCP}$ anion binding sites at the membrane surface is $1.5 \times 10^{17} \mathrm{~m}^{-2}$, which corresponds to approximately 10 phospholipid molecules involved in binding of one PCP anion. This result is consistent with the concept of binding that assumes penetration of PCP anions into 
the bilayer membrane and localized distortion of the lattice that extends over the area of approximately 10 phospholipid molecules.

\section{EXISTENCE OF KINETIC LIMITATIONS}

According to the barrier concept of membrane transport the
transmembrane ion flux increases with voltage according to
sinh(zeV/2kT) which results in the following dependence of membrane
conductance with voltage;

$$
G(V) / G(0)=(2 \mathrm{kT} / \beta z e V) \sinh (\beta z e V / 2 k T)
$$

The empirical coefficient $B$ has been introduced "a posteriori" to indicate that only a fraction, BV of the applied voltage drives the transmembrane transport. Although the physical origin of factor $B$ is not understood, it is a fact that a great variety of membrane conductivity data is in agreement with the barrier model for $\beta$ between 0.7 and $0.9(38,58)$. Taking this evidence into consideration one can show, as has been done in Chapter III, that for both dimer kinetic schemes the steady state normalized conductance is approximately given by (Eqs. 237,312 )

$$
\frac{G(V)}{G(0)}=\frac{2 k T}{B e V} \frac{\left(1+E_{0}\right) \sinh (B e V / 2 k T)}{1+E_{0} \cosh (B e V / 2 k T)} .
$$

The significance of the kinetic limitation parameter $E_{0}$ is that it is related to the displacement of density of membrane permeable ions from the equilibrium value. The kinetic limitation parameter $\mathrm{E}_{0}$ contains two groups of terms; one term which is independent of concentration of uncoupler and $\mathrm{pH}$ of solution, representing a limitation imposed by the 
slow rate of dissociation of dimers $\mathrm{HA}_{2}^{-}$, and the second term which is proportional to the density of uncoupler adsorbed at the membrane surface, representing kinetic limitation associated with the return pathway of transport cycle and with the interfacial processes (Table II). In Figs. 52,54-56 we presented the experimental steady state normalized membrane conductance data, $G(V) / G(0)$, that illustrate the absence, existence, and development of kinetic limitation conditions of membrane transport induced by PCP, 2,4,5-TCP and PCBT. These experimental results are in qualitative agreement with the predictions based on the dimer kinetic schemes.

The results also indicate that for pentachlorophenol the kinetic limitations are rather insignificant (Fig. 52); there appears to be some kinetic Iimitation effect, but it is comparable to the experimental error.

For 2,4,5-trichlorophenol j.t was possible to demonstrate the kinetic limitation only at the highest 2,4,5-TCP concentration used in the experiment. The results clearly indicate that the kinetic limitation becomes progressively developed at higher $\mathrm{pH}$. This result agrees with the predictions of the dimer kinetic scheme that implies interfacial hydrogen ion transfer; this latter model predicts that the kinetic limitation parameter increases with the concentration of uncoupler and with the $\mathrm{pH}$.

In Chapter III, (dimer model A) it was shown that the development of kinetic limitation at low hydrogen ion concentration exists when the transmembrane transport of charge becomes limited by that part of the kinetic scheme that involves neutral HA molecules. In terms of the above model such a limitation can be caused either by the slow rate of backflow of HA molecules across the membrane (kinetic limitation 
measured by the parameter $E_{1}$ in Eq. 227), or by the slow rate of release of protons across the interface (Kinetic limitation measured by the parameter $E_{2}$ in Eq. 230). Our steady state experimental results do not make it possible to distinguish between these two alternatives.

In contrast, for PCBT induced membrane conductance three types of kinetic limitations were observed. One was a gradual development of kinetic limitation effect with the increasing concentration of PCBT in the membrane (Fig. 56). Second and third were the kinetic limitations at $\mathrm{pH}$ below and above the $\mathrm{pH}$ corresponding to conductance maximum $\mathrm{pH}_{\text {gmax }}$ (Fig. 55). The observation of kinetic limitation at $\mathrm{pH}<\mathrm{pH}_{\text {gmax }}$ is interesting in view of the fact that it indicates the presence of an additional kinetically limiting pathway, in this case associated with the PCBT anions. We have concluded that existence of kinetic limitation at low $\mathrm{pH}$ is not compatible with the kinetic scheme involving simple hydrogen ion transfer across membrane/water interface (dimer model A described in chapter III). This new kinetic limitation at low $\mathrm{pH}$ requires a kinetic scheme in which it is assumed that the association/ dissociation reaction $\mathrm{HA}=\mathrm{A}^{-}+\mathrm{H}^{+}$takes place in the aqueous region of membrane/water interface.

The observation of kinetic limitation associated with hydrogen ion transfer at the interface opens a new possibility to study such processes. It is to be expected that interfacial hydrogen ion transfer is facilitated by proper alignment of $H A$ and $A^{-}$species at the membrane/water interface. Favorable alignment may be realized in the case of chlorinated phenols, but not with PCBT, since the experimental results on chlorinated phenols are compatible with the dimer kinetic scheme involving interfacial hydrogen ion transfer whereas PCBT is not. 
If the alignment of $\mathrm{HA}$ and $\mathrm{A}^{-}$species at the interface is such that the groups with which the hydrogen ion interacts are not in contact with the aqueous phase, either due to different orientation or greater adsorption depth of these groups, direct interfacial hydrogen ion transfer may be less probable. It is conceivable that in this case the probability of desorption of $H A$ and $A^{-}$species from the membrane becomes greater or comparable to the probability of transfer of hydrogen ions across the interface so that the dissociation/association process, $\mathrm{HA}=\mathrm{A}^{-}+\mathrm{H}^{+}$, taking place within the aqueous region of the membrane/water interface may be dominating. We propose that the latter takes place at the PCBT modified membranes. As indicated, this hypothesis is based on our observation of kinetic limitation at low pH. The results of electron density distribution calculations $(81,82)$ done in our laboratory by Julian Hobbs suggest that the total dipole moment of PCBT molecule is in the opposite direction of that for chlorinated phenols.

The observation of kinetic limitation of uncoupler induced conductivity at low $\mathrm{pH}$ is one of the new effects in membrane transport kinetics. A phenomenon related to ours, specifically, anomalous voltage dependence of the relaxation current amplitude at low pH, has been reported for TTFB induced conductance (9). The authors interpreted their observation also as a restricted interfacial hydrogen ion transfer; however, they suggested that in their case it was the electric field that assisted anions $\mathrm{A}^{-}$to attain orientation favorable for hydrogen ion transfer. Our steady state data do not make it possible to discriminate between these two detailed alternative interfacial processes. 
EXISTENCE OF A MINIMUM IN THE NET KINETIC LIMITATION PARAMETER AT $\mathrm{pH}=\mathrm{pK}_{\mathrm{a}}$

According to results shown in Fig. 55, the kinetic limitations of PCBT induced conductance are most evident at high and low pH values. There appear to be a minimum at a $\mathrm{pH}$ corresponding to the conductivity maximum which can be approximated by the $\mathrm{pK}_{\mathrm{a}}$ of PCBT. In this section we argue that the occurrence of such a minimum is consistent with the dimer kinetic scheme B, (dimer model B, Chapter III), and show that the condition of existence of a minimum yields another relationship between kinetic constants.

According to dimer model B (Chapter III), the kinetic limitation which increases with the decrease of $\mathrm{pH}$ is that associated with the interfacial transfer of anions and can be written in the form

$$
E_{4}=\frac{h}{C \cdot h+D}
$$

where $\quad h=a_{H} /\left(k_{D} / k_{R}\right)$,

$$
C=\frac{1}{N_{0}}\left(1+\frac{k_{m a}^{H A}}{k_{a m}^{H A}} \frac{k_{B}}{k_{\alpha}} \frac{k_{m a}^{A}}{k_{0}},\right.
$$

and

$$
D=\frac{1}{N_{0}} \frac{k_{m a}^{H A}}{k_{a m}^{H A}}\left(1+\frac{k_{a m}^{A}}{k_{a m}^{A}}\right) \frac{k_{B}}{k_{\alpha}} \frac{k_{m a}^{A}}{k_{0}} \text {. }
$$

The other kinetic limitations which increase with $\mathrm{pH}$ can be written as

$$
E=\frac{1}{A+B \cdot h} \text {, }
$$


where $\quad A=\frac{1}{N_{0}}\left(1+\frac{k_{m a}^{A}}{k_{a m}^{A}}\right) \frac{k_{B}}{k_{\alpha}} \frac{1}{k_{0}}\left(\frac{2}{k_{S}}+\frac{k_{a m}^{H A}}{k_{m a}^{H A}} \frac{1}{k_{D}}+\frac{1}{k_{m a}^{H A}}\right)^{-1}$,

and $\quad B=\frac{1}{N_{0}} \frac{k_{m a}^{A}}{k_{a m}^{A}}\left(1+\frac{k_{a m}^{H A}}{k_{m a}^{H A}}\right) \frac{k_{B}}{k_{\alpha}} \frac{1}{k_{0}}\left(\frac{2}{k_{S}}+\frac{k_{a m}^{H A}}{k_{m a}^{H A}} \frac{1}{k_{D}}+\frac{1}{k_{m a}^{H A}}\right)^{-1}$.

In order that the total kinetic limitation parameter $E_{0}=E+E_{4}$ exhibits a minimum at a certain value of $\mathrm{pH}$, we require that $\mathrm{dE}_{0} / \mathrm{dh}=0$.

This yields

$$
\frac{D}{(C \cdot h+D)^{2}}=\frac{B}{(A+B \cdot h)^{2}}
$$

Since the minimum kinetic limitation occurs at the maximum of conductance, we assume, as a first approximatic n, that $h=1$, which corresponds to $\mathrm{pH}=\mathrm{pK}_{\mathrm{a}}$. It follows that

$$
\frac{(A+B)^{2}}{(C+D)^{2}}=\frac{B}{D}
$$

Substituting for $A, B, C$, and $D$ one arrives at the condition that the rate constants must satisfy in order that a minimum in the net kinetic limitation parameter exists at $\mathrm{pH}=\mathrm{pK}$. This condition is given below.

$$
\frac{2}{k_{S}}+\frac{k_{a m}^{H A}}{k_{m a}^{H A}} \frac{1}{k_{D}}+\frac{1}{k_{m a}^{H A}}=\frac{\left(\frac{1}{k_{m a}^{A}}+\frac{1}{k_{a m}^{A}}\right)}{\left(1+\frac{k_{m a}^{H A}}{k_{a m}^{H A}}\right)}
$$




\section{MODIFICATION OF $2,4,5-T C P$ INDUCED MEMBRANE CONDUCTIVITY BY TETRAPHENYLARSONIUM AND SALICYLATE IONS}

We have shown experimentally that membrane conductance induced by 2,4,5-TCP can be either decreased by the presence of negatively charged salicylate ions or increased by positively charged tetraphenylarsonium ions (Figs. 60,62). There is no doubt that the observed membrane conductance is due to $2,4,5-\mathrm{TCP}$ and not due to modifying ions, because the characteristic $\mathrm{pH}$ dependence of uncoupler induced conductivity remains the same in the presence of both tetraphenylarsonium and salicylate (Fig. 62). Mclaughlin (64) has shown earlier that the effect of salicylate on membrane ion transport is primarily electrostatic; salicylate ions adsorb at the membrane/water interface and decrease the electric potential of both the membrane surface and membrane interior. From studies of interaction of tetraphenylarsonium with membranes (63) one can conclude that the tetraphenylarsonium ions impart positive charge to membranes. The effect of the presence of membrane charge mudifying ions can be conveniently discussed in terms of electric potential distribution illustrated in Figs. 68 and 69 . These figures show the electric potential profile across the membrane and adjacent aqueous layers, assuming that the additional charge due to modifier ions is evenly distributed at the membrane surface. The generalized, potential energy profile for membrane permeable ion diffusing across the membrane is shown in Fig. 70 (see Fig. 8). If the transmembrane ion transport is limited by the diffusion across the membrane barrier, which we have shown is the case for PCP, 2,4,5-TCP, and PCBT at low concentration, then the steady state zero voltage conductance is approximately proportional to a Boltzmann factor having a membrane barrier height in the exponent, 


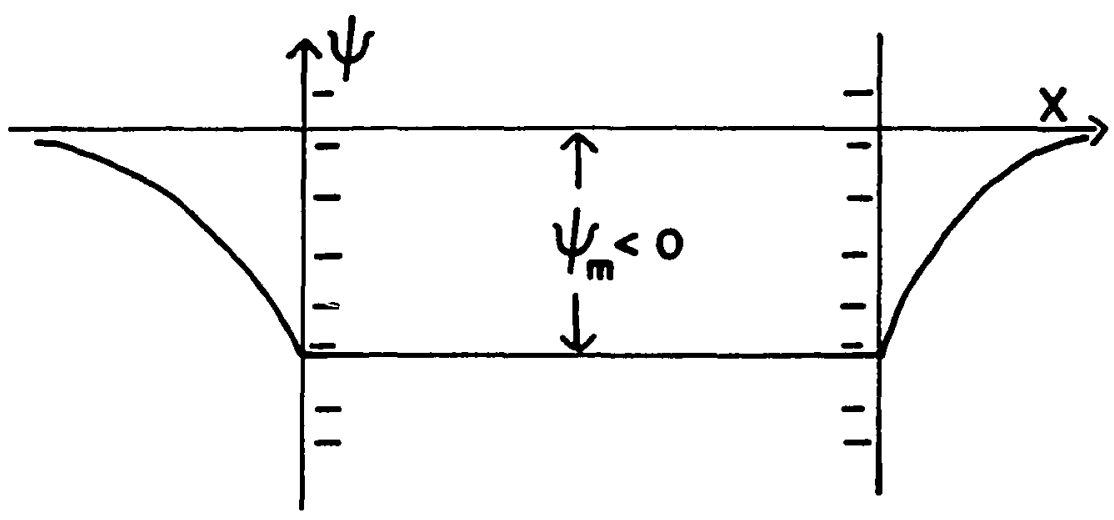

Figure 68. Potential energy profile in the membrane and in the adjacent aqueous layers due to uniformly distributed negative surface charge alone.

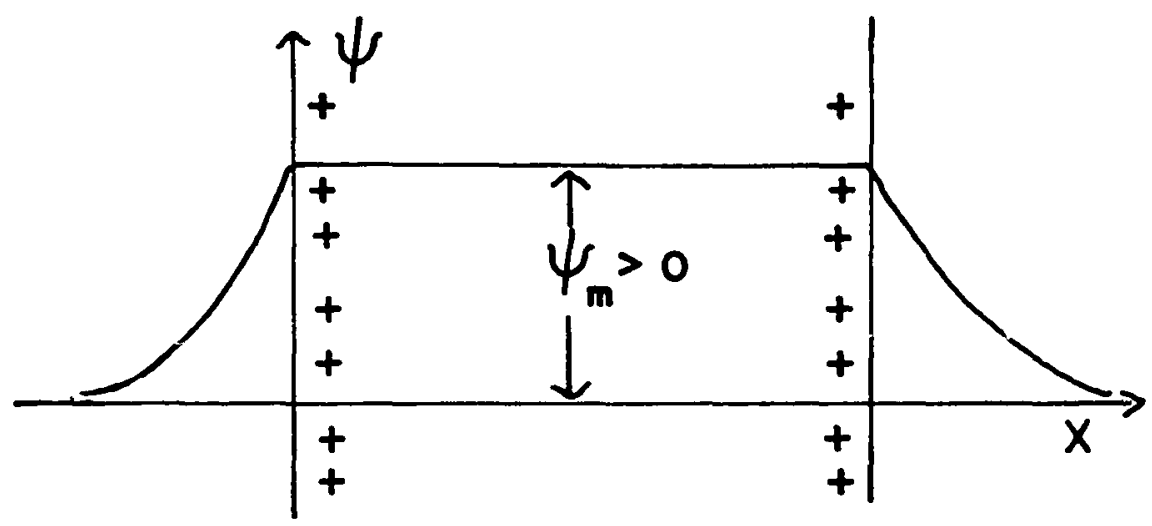

Figure 69. Potential energy profile in the membrane and in the adjacent aqueous layers due to uniformly distributed positivesurface charge alone. 


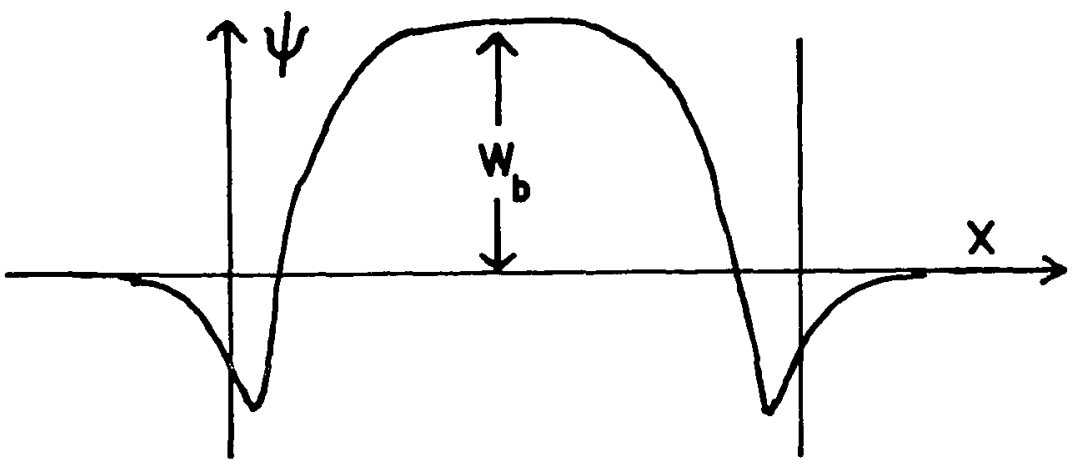

Figure 70. Generalized potential energy profile for membrane permeable ion diffusing across the membrane (see aiso Fig. 8).

$$
G(0) \propto \exp \left(-W_{b} / k T\right)
$$

If $W_{b}$ corresponds to membrane barrier height in the absence of membrane modifying ions, then in their presence the membrane barrier height is changed by $z e \Psi_{m}$, where $\Psi_{m}$ is the membrane potential due to modifier ions (Figs. 68 and 69). Thus the new conductance level G' can be related to the conductance $G$ according to the equation $(7,28,64)$

$$
G^{\prime}=G \cdot \exp \left(-z e \Psi_{m}^{/ k T}\right)
$$

From this relationship one can draw some conclusions about the charge of the membrane permeable ion, ze. Since an increase of membrane conductance was observed in the presence of tetraphenylarsonium ions $(\Psi>0)$, and a decrease for salicylate ions $\left(\Psi_{\mathrm{m}}^{<0}\right)$, the above electrostatic argument compels us to conclude that the 2,4,5-TCP induced membrane conductivity has to be associated with the transmembrane transfer of charge of negative polarity. This evidence provides support to the applicability of kinetic schemes of uncoupler induced charge transport that assume the existence of a negatively charged dimer complex as the membrane permeable ion. 
One of the consequences of altered membrane surface potential by the adsorption of lipophilic ions is that the molar ratio of the neutral and ionized forms of uncoupler is expected to change compared to that in their absence. This change was expected to be demonstrated by not only changes in the magnitude of the conductance, but also in the displacement of the conductance against $\mathrm{pH}$ curve along the pH axis. Our experimental results indicate existence of small $\mathrm{pH}$ shifts of conductance maximum. (Fig. 62).

The problem of changes of uncoupler induced membrane conductance by secondary lipophilic ions can be approached using the assumption that the dominant effect is electrostatic. We have shown that the adsorption of the anionic form of PCP at membrane surface is affected by repulsion between the bound and free PCP anions; the distribution of PCP anions between the membrane surface and the aqueous phase can be accounted for in terms of Langmuir adsorption isotherm corrected for the electric potential in front of membrane surface (Fig. 59). For the purpose of semiquantitative discussion of $\mathrm{pH}$ shifts of conductance maximum we assume that all of the uncoupler and secondary ion binding sites on membrane surface are equivalent. Using subscript "S" for the secondary membrane modifying ions, the membrane surface density of uncoupler anion $\mathrm{A}^{-}$is given by

$$
B_{A}=K^{A}\left(B_{T}-B_{A}-B_{H A}-B_{S}\right) \frac{C_{T}}{1+h} \exp \left(e \Psi_{m} / k T\right)
$$

where

$$
\begin{aligned}
& \mathrm{B}_{\mathrm{X}}=\text { density of bound } \mathrm{X} \text { species, } \\
& \mathrm{K}^{\mathrm{X}}=\text { binding constant of } \mathrm{X} \text { species, } \\
& \mathrm{B}_{\mathrm{T}}=\text { total density of binding sites, }
\end{aligned}
$$


$\mathrm{C}_{\mathrm{T}}=$ total concentration of uncoupler in the aqueous phase, $\Psi_{\mathrm{m}}=$ membrane surface potential,

and $\quad h=a_{H} /\left(k_{D} / k_{R}\right)$.

Similarly for the neutral uncoupler molecule $H A$,

$$
B_{H A}=K^{H A}\left(B_{T}-B_{A}-B_{H A}-B_{S}\right) \frac{h}{1+h} C_{T} \text {. }
$$

Eqs. 347 and 348 enable $B_{A}$ and $B_{H A}$ to be expressed in the forms

$$
B_{H A}=\frac{h}{A+B \cdot h} \text {, }
$$

$$
B_{A}=\frac{1}{C+D \cdot h}
$$

where $\quad A=\frac{1+K^{A} C_{T} \exp \left(e \psi_{m} / k T\right)}{K^{H A} C_{T}\left(B_{T}-B_{S}\right)}$,

$$
B=\frac{1+K^{H A} C_{T}}{K^{H A} C_{T}\left(B_{T}-B_{S}\right)},
$$

$C=\frac{1+K^{A} C_{T} \exp \left(e^{\psi} m^{/ k T}\right)}{K^{A} C_{T}\left(B_{T}-B_{S}\right) \exp \left(e \Psi_{m} / k T\right)}$,

and

$$
D=\frac{1+K^{H A} C_{T}}{K^{A} C_{T}\left(B_{T}-B_{S}\right) \exp \left(e \Psi_{m}^{\Psi} / k T\right)} .
$$

In order to simplify the analysis, it was assumed that $\psi_{\mathrm{m}}$ and $\mathrm{B}_{\mathrm{S}}$ remain constant so that $A, B, C$, and $D$ are constant. Since according to the dimer models, the zero voltage conductance is proportional to the 
product of $\mathrm{B}_{\mathrm{A}}$ and $\mathrm{B}_{\mathrm{HA}}$, the $\mathrm{pH}$ of the conductivity maximum is obtained from the equation

$$
\frac{d}{d h}\left(B_{A} \cdot B_{H A}\right)=0 \text {. }
$$

This yields

$$
h=10^{\left(p K_{a}-p H_{g \max }\right)}=\frac{1+K^{A} C_{T} \exp \left(e_{m}^{\Psi} / k T\right)}{1+K^{H A} C_{T}} .
$$

Our experimental results obtained in the absence of secondary ions and at low concentration of 2,4,5-TCP (i.e. under conditions of minimum kinetic limitations and minimum membrane surface potential changes), indicated that the conductance maximum occurred at pH equal to $\mathrm{pK}_{\mathrm{a}}(=7.0)$ of $2,4,5-\mathrm{TCP}$ (Fig. 62). The prediction of the above model for the occurrence of maximum at $\mathrm{pH}=\mathrm{pK}_{\mathrm{a}}$ and under the condition of $\mathrm{B}_{\mathrm{S}}$ $=0$ when $\left|\Psi_{\mathrm{m}}\right|<<\mathrm{kT} / \mathrm{e}$ is that the binding constants of the ionized and the neutral form of uncoupler $K^{A}$ and $K^{H A}$ are equal. The conductance maximum would be found at a $\mathrm{pH}$ below $\mathrm{pK}_{a}$ if

$$
\exp \left(e \Psi_{m} / k T\right)>K^{H A} / K^{A}
$$

If $\mathrm{K}^{\mathrm{A}}=\mathrm{K}^{\mathrm{HA}}$ remains valid in the presence of secondary ions, then the above inequality reduces to the condition that $\Psi_{m}>0$. Thus the shift of conductance maximum to $\mathrm{pH}_{\mathrm{ph}}$ is expected for a positively charged membrane surface. This kind of $\mathrm{pH}$ shift was observed in the presence of positively charged tatraphenylarsonium ions, in agreement with the above model. For negatively charged salicylate icns a corresponding pH displacement of conductance maximum to $\mathrm{pH}_{\mathrm{pk}}$ was not experimentally observed. The origin of the discrepancy is not clear. It is very likely 
that our model of membrane/water interface is incomplete. First of all, the applicability of the concept of smeared charge has to be tested $(41,83)$. It is expected that if the polarization of the aqueous phase by membrane bound ions was taken into account and the ions were treated as discrete charges one would predict large changes of electric potential in the membrane core, whereas the change of potential within the adsorption plane would be small. The consequence would be a relatively large change of membrane conductance since it is affected by the change of the height of membrane barrier in the core region, whereas the $\mathrm{pH}$ related changes such as density of membrane permeable ions, would be rather small, because they are determined only by the fringe field of membrane bound ions and counterions screening the membrane bound charges. The membrane/water interface region, in contrast to membrane core, is poorly understood and should become the focus of future studies.

\section{TRANSIENT MEMBRANE CURRENTS}

In this section we present arguments to demonstrate that:

(a) The observed changes of the relaxation time constant and the relaxation amplitude with experimentally controllable quantities such as applied potential difference, $\mathrm{pH}$, and concentration of uncoupler agree qualitatively with the predictions of dimer models discussed in Chapter III.

(b) In contrast to PCP and 2,4,5-TCP treated membranes the relaxation characteristics of $\mathrm{PCBT}$ containing membranes indicate that PCBT induced charge transport is strongly kinetically limited, which supports the conclusions made from the voltage dependence of steady state membrane conductance. 
(c) The kinetic limitations of PCP, PCBT, and 2,4,5-TCP induced transmembrane ion flux is not caused by slow kinetics of formation of membrane permeable ions, $\mathrm{HA}+\mathrm{A}^{-}=\mathrm{HA}_{2}^{-}$.

It follows from the theoretical analysis of kinetic schemes of membrane transport that the relaxation amplitude $\alpha$ of transient membrane current is proportional to the kinetic limitation parameter E, and is given by $\alpha=E \cosh (\mathrm{eV} / 2 \mathrm{kT}$ ) (Eqs. 244,323). This expression provides the desired connection between the steady state studies of kinetic limitations, in which $E$ is obtained from measurements of voltage dependence of steady state conductance, and the transient current studies, which provide relaxation amplitude. Thus, the greater the kinetic limitation, the greater the relaxation amplitude. Furthermore, if our understanding of uncoupler induced membrane conductance is conceptually correct, then both $\alpha$ and $E$ are expected to have the same functional dependence on $\mathrm{pH}$ and uncoupler concentration. These relationships were confirmed by our numerical solutions of the sets of differential equations describing the kinetic schemes.

We have found that the kinetic limitation of PCP, PCBT, and $2,4,5-\mathrm{TCP}$ induced membrane transport is not associated with the slow dimerization process. Briefly, the argument goes as follows: The analysis of dimer models has shown that in case of the kinetic limitation associated with the slow dimerization process both the relaxation amplitude and the time constant are independent of $\mathrm{pH}$ and uncoupler concentration. This contrasts with our experimental results for PCP, PCBT, and 2,4,5-TCP treated membranes showing that the time constant is dependent on both the $\mathrm{pH}$ and the uncoupler concentration. Therefore, the process of formation and dissociation of membrane permeable dimers, 
$\mathrm{HA}_{2}^{-}$, does not limit the transmembrane ion flux induced by the uncouplers PCP, PCBT, and $2,4,5-T C P$.

With the exclusion of the dimerization kinetics as the origin of observed kinetic limitations, there are several other kinetically limiting processes that may produce the observed experimental results. Some are common to both dimer schemes. These are: (1) slow return pathway of $\mathrm{HA}$ molecules, and (2) slow rate of the reaction $\mathrm{HA}^{-} \mathrm{A}^{-}=$ $\mathrm{H}^{+}$, which is either due to slow rate of dissociation of $\mathrm{HA}$ form, or slow association of hydrogen ion from the aqueous phase. For dimer model $B$ there is, in addition, a possibility of slow rate of desorption of HA molecules from the membrane.

PCP, PCBT, and 2,4,5-TCP induced transient current measurements have shown that membrane current relaxations were enhanced at pH values above the $\mathrm{pH}$ of maximum conductance. The theoretical analysis of kinetics of dimer transport schemes indicated that the above observation is consistent with either the kinetic Iimitation due to slow backflow of HA form, or due to slow interfacial transfer of hydrogen ions, or for the case of PCBT, due to slow interfacial transfer of HA molecules. Furthermore, the dependence of relaxation characteristics on concentration of uncoupler is also similar for the above types of kinetic limitations. From the limited relaxation data it is not possible to determine which type of kinetic limitation is responsible for the current relaxations observed at high $\mathrm{pH}$.

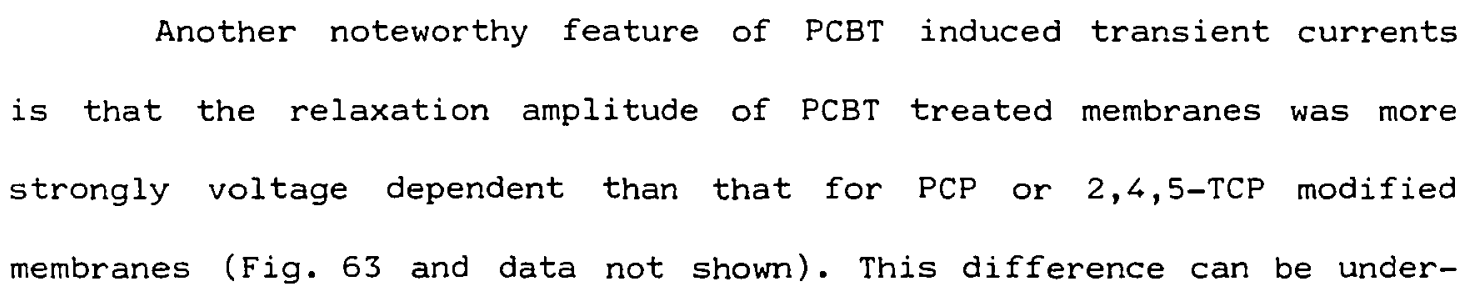


stood and is consistent with the predictions of kinetic schemes. It follows from the relationship between the relaxation amplitude $\alpha$ and the kinetic limitation parameter $E$ that for a kinetically limited membrane transport system $\Delta \alpha=E\left[\cosh \left(\mathrm{eV}_{2} / 2 \mathrm{kT}\right)-\cosh \left(\mathrm{eV}_{1} / 2 \mathrm{kT}\right)\right]$. Our observation of greater changes of the magnitude of PCBT inciuced relaxation amplitude $\Delta \alpha$ with the applied voltage $V$, compared to that of PCP and 2,4,5-TCP, is consistent with our conclusion made earlier that PCBT induced charge transport is more strongly kinetically limited (large E) than that of PCP and 2,4,5-TCP (small E). The existence of larger relaxation amplitude and strong kinetic limitation in the case of PCBT treated membranes is also consistent with the fact that, for a given concentration of uncoupler, the PCBT induced membrane conductance is also greater compared to that for PCP (compare Fig. 42 with Fig 43 ). Naturally, for greater rate of transport of charge across the membrane it is more likely that other processes which are coupled to ion translocation may not be fast enough to maintain the distribution of transport constituents at or close to equilibrium. This can be seen also in the results of analysis of kinetic schemes; both the conductance (Eqs. 238,313) and the kinetic limitation parameters (Eqs. 227, $230,298,301-303)$ are proportional to $k_{0} k_{\alpha} / k_{B}$, the product of the rate constant of dimer translocation across the membrane and the dimer association constant $k_{\alpha} / k_{B}$. Therefore, if the rate of ion transport across the membrane is increased either due to the increase of $\mathrm{k}_{0}$, and/or due to greater dimer association constant $k_{\alpha} / k_{\beta}$, then, if other properties remain unaffected, it is to be expected that such a system is kinetically limited to a greater degree. The experimental results for PCBT-induced conductance and relaxation are consistent with the 
above statements. The representative value of $k_{0} k_{\alpha} / k_{B}$ for PCP induced conductance, obtained from the $\mathrm{pH}$ dependence and concentration dependence of zero voltage conductance, is of the order of $1 \times 10^{14} \mathrm{~cm}^{2} / \mathrm{M} . \mathrm{sec}$, whereas that for the case of PCBT conductance, which is strongly kinetically limited, it is of the order of $1 \times 10^{16} \mathrm{~cm}^{2} / \mathrm{M} . \mathrm{sec}$. For comparison, from the results of kinetic studies of uncoupler DTFB reported by Cohen et. al. (8), we estimate $k_{0} k_{\alpha} / k_{B} \simeq 3 \times 10^{14} \mathrm{~cm}^{2} /$ M.sec. which is about the same order of magnitude as for PCP.

The kinetic limitation of PCBT induced conductance observed in steady state studies at low pH was attributed to slow interfacial transfer, or desorption, of PCBT anions. We have not been able to observe with our equipment a relaxation process in the low $\mathrm{pH}$ range. This negative result is also consistent with the predictions made from dimer kinetic scheme B (dimer model B: Chapter III); from the $\mathrm{pH}$ dependence of relaxation characteristics (Fig. 39), it follows that, in general, the relaxation time becomes shorter at lower $\mathrm{pH}$ and may be below the resolving power of the existing experimental setup.

In summary, the qualitative agreement of transient current characteristics for PCP, PCBT, and 2,4,5-TCP modified membranes with those obtained from the steady state studies and properties of dimer kinetic schemes indicate that our understanding of the mechanism of ion transport induced by these compounds is conceptually correct.

\section{BIOLOGICAL SIGNIFICANCE}

In this section we will address three major results

(a) the observed correlation between the $\mathrm{pH}$ dependence of PCBT induced membrane conductivity and the PCBT induced uncoupling activity in mitochondria, 
(b) the toxic effect of PCP on alga Selenastrum capricornutum occurring at the same PCP concentration level as the appearance of induced electrical conductivity of lipid bilayer membranes, and

(c) the identity of mechanism of PCP, PCBT, and 2,4,5-TCP induced electrical conductivity in artificial membranes and their uncoupling activity in ATP producing organelles.

We have explored the problem of existence of a causal relationship between the induced membrane conductivity and toxicity of weak acid uncouplers primarily for the following reasons. First, because PCP, PCBT, and 2,4,5-TCP have the characteristics of weak acid uncouplers. Second, because of the possibility to develop future studies of uncoupling at the quantitative level if the identity of the mechanism of uncoupling and induced electrical conduction is established.

We restate briefly the conception of the problem that existed at the time when this project was formulated. There were two major conflicting points of view of uncoupling process. According to one school of thought the primary effect of uncouplers was due to binding of uncoupler to proteins involved in the conversion of ADP to ATP $(33,84)$; the electrical conductivity induced in lipid membranes was considered to be either an artifact or a phenomenon of secondary importance. The alternative hypothesis assumed that the primary action of weak acid uncouplers is their ability to dissipate the electrochemical potential energy accumulated in the hydrogen ion concentration gradients and electric charge separation existing across energy transducing membrane (11). This latter point of view is consistent, or considered as a corollary of the chemiosmotic hypothesis of coupling electron transport to phosphorylation $(2,85)$. 
PCBT was one of the compounds used in the arguments against the applicability of the second hypothesis (34). The investigators making such claims succeeded in demonstration and characterization of uncoupling activity of PCBT. However, their data on PCBT induced electrical conductivity in lipid membranes convinced them that there is no correlation between those two effects (34). In contrast, we have found with carefully controlled experiments, that PCBT induces relatively large electrical conductivity in lipid membranes. Furthermore, we have found that the $\mathrm{pH}$ dependence of the induced membrane conductance and the $\mathrm{pH}$ dependence of PCBT uncoupling activity in rat liver mitochondria, given in their report, are very similar.

Another set of experiments on uncoupling activity, the reduction of carbon uptake of alga Selenastrum capricornutum by PCP has also provided support to the point of view that the origin of PCP toxicity, as we observed it, is physical in nature. We have found evidence for rapid depletion of the energy source, ATP, that is required for carbon uptake by the alga. We have found that the onset of toxic effect is very rapid; it occurred on a time scale of an hour, which contrasts to toxic effects related to the changes of algal metabolism occurring on a time scale of 10 hours. Even more important is the observation that the toxic effect in alga occurred at the same concentration level of PCP at which we observed appearance of PCP induced electrical conductivity in lipid membranes.

The biological significance of our results is seen in the following. We have demonstrated the existence of PCBT induced electrical conductivity in lipid membranes, and thus eliminated one of the uncouplers from the list for which this effect, paralleling uncoupling, 
was disputed. Further, we have shown that for PCP, the onset of toxicity in alga Selenastrum capricornutum is parallel to the onset of PCP induced conductivity in membranes. These effects are consistent with and provide support to the hypothesis that the mechanism of uncoupling and the mechanism of induced conductivity by weak acid uncouplers are identical (8). As additional support for the chemiosmotic theory of uncoupling action, we refer to the results of our studies of membranes treated with PCBT, PCP or 2,4,5-TCP by electrical methods. We have found that the flow of electric charge across the membrane is supported by the diffusion of hydrogen ions on the aqueous side of the membrane/water interface, and that the transfer of charge across the membrane core is due to the uncoupler related anion. The transfer of charge in the membrane is kinetically coupled to the translocation of protons in the opposite direction. The results obtained in this project provide evidence that strongly supports the view that the physical processes accompanying the dissipation of hydrogen ion electrochemical potential energy in phosphorylating membranes by weak acid uncouplers and those associated with the effect of induced electrical conductivity are indeed identical. 


\title{
CHAPTER VI
}

\section{CONCLUSIONS}

\begin{abstract}
Pentachlorobenzenethiol was one of the compounds used in the past to point out the absence of correlation between the phenomenon of uncoupling of oxidative phosphorylation and induced membrane conductivity. We have experimentally demonstrated the existence of the disputed membrane conductivity due to pentachlorobenzenethiol and have also shown that the $\mathrm{pH}$ dependence of pentachlorobenzenethiol-induced membrane conductance was similar to the $\mathrm{pH}$ dependence of uncoupling activity in rat liver mitochondria. The correlation between (a) the pentachlorobenzenethiol-induced membrane conductance and the pentachlorobenzenethiol-induced uncoupling activity, and (b) the concentration of pentachlorophenol required to induce conductivity in membranes and the concentration required to induce toxicity in alga are consistent with the chemiosmotic coupling hypothesis.

Out of a series of kinetic models of membrane transport studied in this project the one that best explains the experimental results of membrane conductivity induced by pentachlorophenol, pentachlorobenzenethiol, and 2,4,5-trichlorophenol is the one in which the induced conductance is the result of flow of the dimer complex $\mathrm{HA}_{2}^{-}$across the membrane. This transmembrane flux of $\mathrm{HA}_{2}^{-}$ions is supported by the backflow of neutral uncoupler molecules HA across the membrane. The results of the study of kinetic limitations of membrane conductivity induced by the above compounds are consistent with the hypothesis that the trans-
\end{abstract}


membrane ion flux is kinetically coupled with the transfer of hydrogen ions across the membrane. The continuity of charge flow across the membrane is maintained by the flow of protons across the membrane water interface in the case of pentachlorophenol and 2,4,5-trichlorophenol, and by the interfacial flow of pentachlorobenzenethiol anions for the case of pentachlorobenzenethiol.

The study of adsorption of pentachlorophenol anions to Iipid bilayers indicates that this phenomenon can be satisfactorily explained by the Langmuir adsorption isotherm, provided that the electrostatic repulsion between the anions adsorbed to the membrane and those adjacent to the membrane is accounted for by a Boltzmann factor.

The changes of the magnitude of 2,4,5-trichlorophenol induced membrane conductivity in the presence of tetraphenylarsonium and salicylate ions can be explained by electrostatic arguments by taking into consideration adsorption of these membrane modifier ions at the membrane surface and the change of the height of membrane energy barrier for membrane permeable ions derived from 2,4,5-trichlorophenol. However, this electrostatic argument was found inadequate to explain the pH shift of conductivity observed in the presence of salicylate ions. 


\section{REFERENCES}

1. Boyer P. D., Cross R. L., Momsen W., 1973. A new concept for energy coupling in oxidative phosphorylation based on a molecular explanation of the oxygen exchange reaction. Proc. Nat. Acad. Sc. (U.S.) $70: 2837$.

2. Mitchell P., 1966. Chemiosmotic coupling in oxidative and photosynthetic phosphorylation. Biol. Rev. 41:445.

3. Hinkle P. C., McCarty R. E., 1978, March. How cells make ATP. Sci. Am. 238:104.

4. Terada H., 1975. Some biochemical and physicochemical properties of the potent uncoupler SF 6847 (3,5-di-tert-butyl-4-hydroxybenzylidenemalononitrile). Biochim. Biophys. Acta. 387:519.

5. LeBlanc O. H., 1971. The effect of uncouplers of oxidative phosphorylation on lipid bilayer membranes: carbonylcyanide m-chlorophenylhydrazone. J. Membrane Biol. 4:227.

6. Hopfer U., Lehninger A. L., Lennarz W. J., 1970. The effect of the polar moity of lipids on bilayer conductance induced by uncouplers of oxidative phosphorylation. J. Membrane Biol. 3:142.

7. McLaughlin S., 1972. The mechanism of action of DNP on prospholipid bilayer membranes. J. Membrane Biol. 9:361.

8. Cohen F. S., Eisenberg M., McLaughlin S., 1977. The kinetic mechanism of action of an uncoupler of oxidative phosphorylation. J. Membrane Biol. 37:361.

9. Dilger J., McLaughlin S., 1979. Proton transport through membranes induced by weak acids. A study of two substituted benzimidazoles. J. Membrane Biol. 46:359.

10. Laris P. C., Bahr D. P., Chaffee R. R. J., 1975. Membrane potentials in mitochondrial preparations as measured by means of a cyanine dye. Biochim. Biophys. Acta. 376:415.

11. Mitchell P., Moyle J., 1967. Acid-base titration across the membrane system of rat liver mitochondria. Catalysis by uncouplers. Biochem. J. 104:588.

12. Guidotti G., 1980. Membrane proteins: structure and arrangement in the membrane. In Membrane Physiology. Edited by $T$. E. Andreoli, J. F. Hoffman and D. D. Fanestil. 
13. Jain M. K., 1972. The bimolecular lipid membrane: a system. Van Nostrand Reinhold Company.

14. Tanford C., 1978. The hydrophobic effect and the organization of living matter. Science 200:1012.

15. Edidin M., 1974. Rotational and translational diffusion in membranes. Ann. Rev. Biophys. Bioeng. 3:179.

16. Fahey P. F., Koppel D. E., Barak L. S., Wolf D. E., Elson E. L., Webb W. W., 1977. Lateral diffusion in planar lipid bilayers. Science 195:305.

17. Johnson L. W., Hughes M. E., Zilversmit D. B., 1975. Use of phospholipid exchange protein to measure inside-outside transportations in phosphatidylcholine liposomes. Biochim. Biophys. Acta. $375: 176$.

18. Rothman J. E., Dawidowicz E. A., 1975. Asymmetric exchange of vesicle phospholipids catalyzed by the phosphatidylcholine exchange protein. Measurement of inside-outside transitions. Biochemistry $14: 2809$.

19. Kornberg R., McConell H. M., 1971. Inside-outside transitions of phospholipids in vesicle membranes. Biochemistry 10:1111.

20. Singer S. J., Nicholson G. L., 1972. The fluid mosaic model of the structure of cell membrane. Science 175:720.

21. Palmier J. M., Hall D. O., 1972. The mitochondrial membrane system. Progr. Biophys. Mol. Biol. 24:127.

22. Bangham A. D., Standish M. M., Watkins J. C., 1965. Diffusion of univalent ions across the lamellae of swollen phospholipids. J. Mol. Biol. 13:238.

23. McLaughlin S., Harary H., 1976. The hydrophobic adsorption of charged molecules to bilayer membranes: A test of the applicability of the Stern equation. Biochemistry 15:1941.

24. Korenbrot J. I., 1977. Ion transport in membranes: Incorporation of biological ion-translocating proteins in model membrane systems. Ann. Rev. Physiol. 39:19.

25. Racker E., Stoeckenius W., 1974. Reconstitution of purple membrane vesicles catalyzing light driven proton uptake and ATP formation. J. Biol. Chem. 249:662.

26. Racker E., Kandrach A., 1973. Partial resolution of the enzymes catalyzing oxidative phosphorylation. XXXIX Reconstitution of the third segment of oxidative phosphorylation. J. Biol. Chem. $248: 5841$. 
27. Huang C., 1969. Studies on phosphatidylcholine vesicles. Formation and physical characteristics. Biochemistry $8: 344$.

28. Smejtek P., Hsu K., Perman W. H., 1976. Electrical conductivity in lipid bilayer membranes induced by pentachlorophenol. Biophys. J. $16: 319$.

29. Foster M., Mclaughlin S., 1974. Complexes between uncouplers of oxidative phosphorylation. J. Membrane Biol. 17:155.

30. Neumcke B., Bamberg E., 1975. The action of uncouplers on lipid bilayer membranes. In Membranes Vol. 3, Lipid Bilayers and biological membranes: dynamic properties. G. Eisenman editor p.215. Mercel Dekker, New York.

31. Bakker E. P., Van Don Heuvel E. J., Wiechmann A. H. C. A., 1973. A comparison between the effectiveness of uncouplers of oxidative phosphorylation in mitochondria and in different artificial membrane systems. Biochim. Biophys. Acta. 292:78.

32. Ting H. P., Wilson D. G., Chance B., 1970. Effects of uncouplers of oxidative phosphorylation on the specific conductance of bimolecular lipid membranes. Arch. Biochem. Biophys. 141:141.

33. Hanstein W. G., 1976. Uncoupling of oxidative phosphorylation. Biochim. Biophys. Acta. 456:129.

34. Wilson D. F., Ting H. P., Koppelman M. S., 1971. Mechanism of action of uncouplers of oxidative phosphorylation. Biochemistry. $10: 2897$.

35. McLaughlin S. G. A., Dilger J. P., 1980. The transport of protons across membranes by weak acids. Physiol. Rev. 60:825.

36. Hanstein W. G., Hatefi Y., 1974. Trinitrophenol: a membraneimpermeable uncoupler of oxidative phosphorylation. Proc. Nat. Acad. Sci. (U.S.) $71: 288$.

37. McLaughlin S., Eisenberg M., Cohen F., Dilger J., 1978. The unique ability of picrate to uncouple submitochondrial particles but not mitochondria is consistent with the chemiosmotic hypothesis. In Frontiers of biological energetics, edited by $P$. $L$. Dutton, J. S. Leigh and A. Scarpa. New York Academic press Vol. 2 , p. 1205 .

38. Anderson O. S., Fuchs M., 1975. Potential energy barriers to ion transport within lipid bilayers. Biophys. J. 15:795.

39. Haydon D. A., Hladky S. B., 1972. Ion transport across thin lipid membranes: a critical discussion of mechanisms in selected systems. Quart. Rev. Biophys. 5:187.

40. Neumcke B., Lauger P., 1969. Nonlinear electrical effects in lipid bilayer membranes. II Integration of the generalized Nernst-Plank equation. Biophys. J. 9:1160. 
41. Anderson 0. S., Feldberg S., Nakadomari H., Levy S., McLaughlin S., 1978. Electrostatic interactions among hydrophobic ions in lipid bilayer membranes. Biophys. J. 21:35.

42. Ketterer B., Neumcke B., Lauger P., 1971. Transport mechanism of hydrophobic ions through lipid bilayer membranes. J. Membrane Biol. $5: 225$.

43. Hladky S. B., 1979. The carrier mechanism. Curr. Top. Membranes Transp. 12:53.

44. Finkelstein A., 1970. Weak acid uncouplers of oxidative phosphorylation. Mechanism of action on thin lipid membranes. Biochim. Biophys. Acta. 205:1.

45. Lea E. J. A., Croghan P. C., 1969. The effect of 2,4-dinitrophenol on the properties of thin phospholipid films. J. Membrane Biol. 1:225.

46. Poonia N. S., 1975. Complexation of sodium and potassium with macrocyclic polyethers: Activation of the counter anions. J.Inorg. Nucl. Chem. 37:1855.

47. Neumcke B., 1971. Diffusion polarization at lipid bilayer membranes. Biophysik 7:95.

48. Lucas C. R., Peach M. E., 1970. Reactions of pentachlorothiophenol. 1. Preparation of some simple metaliic and non-metallic derivatives. Can. J. Chem. 48:1869.

49. Sargent D. F., 1976. An apparatus for the measurement of very small membrane relaxation currents. Anal. Biochem. 70:100.

50. Bangham A. D., Hill M. W., Miller N. G. A., 1974. Preparation and use of liposomes as models of biological membranes. Methods Meribr. Biol. $1: 1$.

51. Bangham A. D., Flemans R., Heard D. H., Seaman G. V. F., 1958. An apparatus for microelectrophoresis of small particles. Nature $183: 642$.

52. Stark G., Benz R., 1971. The transport of potassium through lipid bilayer membranes by the neutral carriers valinomycin and monactin. J. Membrane Biol. 5:133.

53. Lauger P., Stark G., 1970. Kinetics of carrier-mediated ion transport across lipid bilayer membranes. Biochim. Biophys, Acta. $211: 458$.

54. Stark G., Ketterer B., Benz R., Lauger P., 1971. The rate constants of valinomycin mediated ion transport through thin lipid membranes. Biophys. J. 11:981. 
55. Benz R., Stark G., Janko K., Lauger P., 1973. Valinomycinmediated ion transport through neutral lipid membranes: Influence of hydrocarbon chain length and temperature. J. Membrane Biol. $14: 339$.

56. Knoll W., Stark G., 1975. An extended kinetic analysis of valinomycin-induced Rb-transport through monoglyceride membranes. J. Membrane Biol. 25:249.

57. Krasne S., Eisenman G., 1976. Influence of molecular variations of ionophore and lipid on the selective ion permeability of membranes: I. Tetranactin and the methylation of nonactin-type carriers. J. Membrane Biol. 30:1.

58. Smejtek P., Paulis-Illangasekare, 1979. Modification of ion transport in lipid bilayer membranes in the presence of 2,4-dichlorophenoxyacetic acid. II. Suppression of tetraphenylborate conductance and changes of interfacial potentials. Biophys. J. $26: 467$.

59. Vetter K. J., 1967. Electrochemical kinetics. Theoretical aspects. Academic Press, New York.

50. Shah D. O., 1970. Surface chemistry of lipids. Adv. Lipid Res. $8: 347$.

61. Hilton B. D., O'Brien R. D., 1973. The effect of DDT and its analogs upon lecithin and other monolayers. Pestic. Biochem. Physiol. 3:206.

62. Overbeek J. T. G., Wiersema P. H., 1967. The interpretation of electrophoretic mobilities. In Electrophoresis: theory, methods and applications M. Bier editor Vol. 2, p. 1. Academic Press, New York.

63. Pickar A. D., Benz R., 1978. Transport of oppositely charged lipophilic probe ions in lipid bilayer membranes having various structures. J. Membrane Biol. 44:353.

64. McLaughlin S., 1973. Salicylates and phospholipid bilayer membranes. Nature 243:234.

65. Stark G., 1980. Negative hydrophobic ions as transport-mediators for positive ions. Evidence for a carrier mechanism. Biochim. Biophys. Acta. 600:233.

66. Werdan K., Heldt H. W., Milovancev M., 1975. The role of $\mathrm{pH}$ in the regulation of carbon fixation in the chloroplast stroma. Studies on $\mathrm{CO}_{2}$ fixation in the light and dark. Biochim. Biophys. Acta $396: 276$. 
67. Heldt H. W., Werdan K., Milovancev M., Geller G., 1973. Alkalization of the chloroplast stroma caused by light dependent proton flux into the thylakoid space. Biochim Biophys. Acta. $313: 224$.

68. Buchel K. H., Korte F., Trebst A., Pistorius E., 1965. Inhibition of photosynthesis reactions by $\mathrm{NH}-a c i d i c$ imidazoles and benzimidazoles. Angew. Chem. Internat. Edit. $4: 789$.

69. Smith F. A., West K. R., 1969. A comparison of the effect of metabolic inhibitors on chloride uptake and photosynthesis in Chara corallina. Aus. J. Biol. Sci. 22:351.

70. Liberman E. A., Topaly V. P., Silberstein A., Okhlobistin 0., 1971. Mobile ion carriers and the negative resistance of membranes. 1. Uncoupling agents of oxidative phosphorylation proton carriers. Biophysics 16:637.

71. Liberman E. A., Topaly V. P., 1968. Selective transport of ions through bimolecular phospholipid membranes. Biochim. Biophys. Acta. 163:125.

72. Ashton F. M., Crafts A. C., 1973. Mode of action of herbicides. Wiley Interscience, New York, p. 256.

73. Weinbach E. C., Garbus J., 1965. The interaction of uncoupling phenols with mitochondria and with mitochondrial protein. J. Biol. Chem. 240:1811.

74. Gaur B. K., Beevers H., 1959. Respiratory and associated responses of carrot discs to substituted phenols. Plant Physiol. 34:427.

75. Weinbach E. C., 1956. Biochemical basis for the toxicity of pentachlorophenol. Science 124:940.

76. Blackman G. E., Parke M. H., Garton G., 1955. The physiological activity of substituted phenols. II. Relationship between physical properties and physiological activity. Arch. Biochem. Biophys. $54: 55$.

77. Blackman G. E., Parke M. H., Garton G., 1955. The physiological activity of substituted phenols. I. Relationship between chemical structure and physiological activity. Arch. Biochem. Biophys. $54: 45$.

78. Weinbach E. C., 1954. The effect of pentachlorophenol on oxidative phosphorylation. J. Biol. Chem. 210:545.

79. Lauger P., Neumcke B., 1973. Theoretical analysis of ion conductance in lipid bilayer membranes. In Membranes Vol. I. Lipid bilayers and antibiotics. G. Eisenman editor p. 1, Marcel Dekker, New York. 
80. Hladky S. B., 1973. The effect of stirring on the flux of carriers into black lipid membranes. Biochim. Biophys. Acta. 307:261.

81. Streitwieser A. Jr., 1961. Molecular orbital theory for organic chemists. John Wiley \& Sons Inc., New York-London-Sydney.

82. Del Re G., 1958. A simple MO-LCAO method for the calculation of charge distribution in saturated organic molecules. J. Chem. Soc. 4051 .

83. Wang C. C., Bruner L. J., 1978. Evidence for a discrete charge effect within lipid bilayer membranes. Biophys. J. 24:749.

84. Wilson D. F., Brooks E., 1970. Inhibition of mitochondrial respiration by hydroxylamine and its relation to energy conservation. Biochemistry 9:1090.

85. Mitchell P., 1961. Coupling of phosphorylation to electron and hyorogen transfer by a chemiosmotic type of mechanism. Nature $191: 144$. 


\section{APPENDIX}

REACTION LAYER THICKNESS

It is assumed that in the case of interfacial transfer of $H A$ and $\mathrm{A}^{-}$species the proton association-dissociation reaction is taking place in the aqueous phase. Since under normal experimental conditions the buffer concentration is several orders of magnitude greater than the concentration of the uncoupler in the aqueous phase, we assume that hydrogen ion concentration is constant, both in space and time.

Consider the interfacial processes occurring at the positively biased membrane surface and depicted in Fig. 71. Due to the applied electric potential difference there is a net rate of transfer of $A^{-}$ ions from the membrane surface into the aqueous phase, and a net rate of entry of HA molecules from the aqueous phase into the membrane. The excess aqueous concentration of $\mathrm{A}^{-}$is dissipated by the recombination with hydrogen ions. It is to be expected that the disturbance is greatest at the interface and that it decreases with the distance from the interface. We define $\gamma$ as the characteristic length for the spatial distribution of $A^{-}$and $H A$ and refer to it as a reaction layer thickness. It can be estimated from the steady-state condition, namely that

$\frac{\partial}{\partial t} n_{H A}=D^{H A} \frac{d^{2}}{d x^{2}} n_{H A}+k_{A} a_{H A} n_{a}-k_{D} n_{H A}=0$,

and $\quad \frac{\partial}{\partial t} n_{A_{a}}=D^{A} \frac{d^{2}}{d x^{2}} n_{A_{a}}-k_{A} a_{H^{N}} N_{a}+k_{D} n_{H A}=0$. 


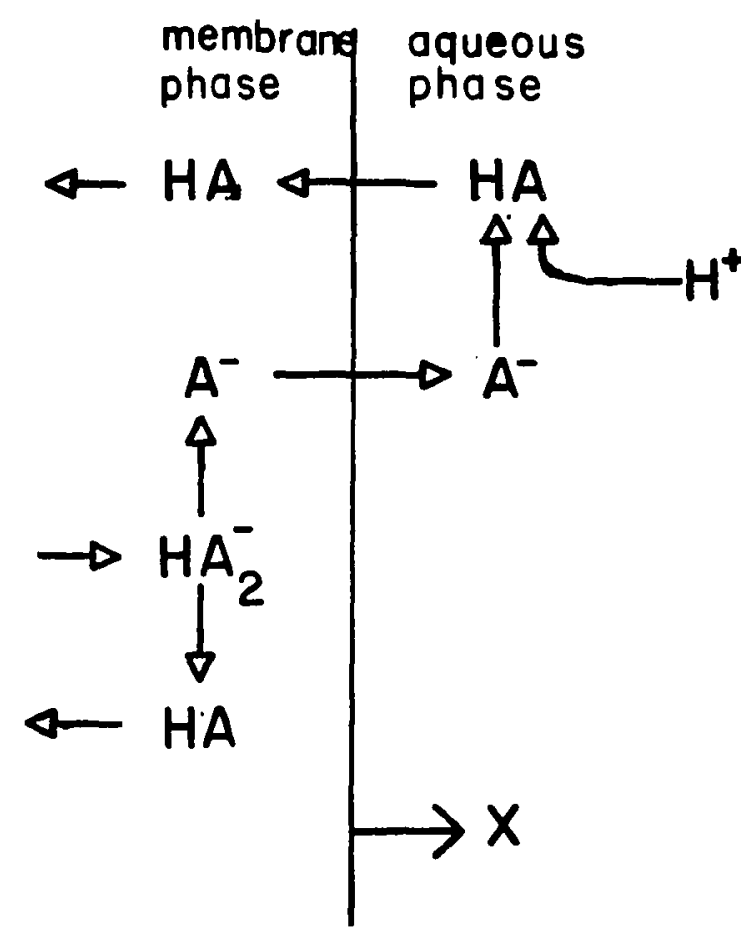

Figure 71. Interfacial processes occurring in dimer model $B$ at the positively biased membrane surface.

In addition, at steady state, the total flux of anions $A^{-}$at any point in the aqueous phase is zero. Therefore

$$
D^{H A} \frac{d}{d x} n_{H A}(x)+D^{A} \frac{d}{d x} n_{A}(x)=0 .
$$

Integrating with the assumption that $D^{H A}=D^{A}=D$ yields

$$
\mathrm{n}_{\mathrm{HA}}(\mathrm{x})+\mathrm{n}_{\mathrm{A}}(\mathrm{x})=\mathrm{N}_{\mathrm{a}} \text {, }
$$

where $\mathrm{N}_{a}$ is the total aqueous concentration of the uncoupler, which is a constant. By eliminating $\mathrm{n}_{\mathrm{A}}$ between Eqs. A.1 and A.4 one can obtain 


$$
D \frac{d^{2}}{d x^{2}} n_{H A}-\left(k_{D}+k_{A} a_{H}\right) n_{H A}+k_{A} a_{H} N_{a}=0 \text {. }
$$

The solution, taking into consideration that ${ }^{n_{H A}}$ is finite at $x+\infty$, is given by

$$
n_{H A}(x)=\frac{h}{1+h} N_{a}-M \exp (-\lambda x),
$$

where $M$ is a constant and

$$
\lambda=\sqrt{\left(k_{D}+k_{A} a_{H}\right) / D}
$$

The corresponding spacial variation of $\mathrm{n}_{\mathrm{A}}$ is

$$
n_{A_{a}}(x)=\frac{1}{1+h} N_{a}+M \exp (-\lambda x)
$$

Hence the reaction layer thickness is given by

$$
\gamma=\frac{1}{\lambda}=\frac{D}{k_{A H} a_{H}+k_{D}} \text {. }
$$

